JOINT TRANSPORTATION RESEARCH PROGRAM

FHWA/IN/JTRP-2007/6

Final Report

INFLUENCE OF SPEED LIMITS ON ROADWAY SAFETY IN INDIANA

Nataliya V. Malyshkina

Fred L. Mannering

Samuel A. Labi

May 2007 
Final Report

FHWA/IN/JTRP 2007/06

\title{
INFLUENCE OF SPEED LIMITS ON ROADWAY SAFETY IN INDIANA
}

\author{
By \\ Nataliya V. Malyshkina, Ph.D. Student \\ Fred L. Mannering, Ph.D., Professor \\ Samuel A. Labi, Ph.D., Assistant Professor \\ School of Civil Engineering \\ Purdue University \\ Joint Transportation Research Program \\ Project No. C-36-59VV \\ File No. 8-5-48 \\ SPR-3030 \\ Conducted in Cooperation with the \\ Indiana Department of Transportation and \\ the U.S. Department of Transportation \\ Federal Highway Administration
}

The content of this report reflects the views of the authors who are responsible for the facts and the accuracy of the data presented herein. The contents do not necessarily reflect the official views or policies of the Indiana Department of Transportations or the Federal Highway Administration at the time of publication. This report does not constitute a standard, specification or regulation.

School of Civil Engineering

Purdue University

May 2007 
TECHNICAL REPORT STANDARD TITLE PAGE

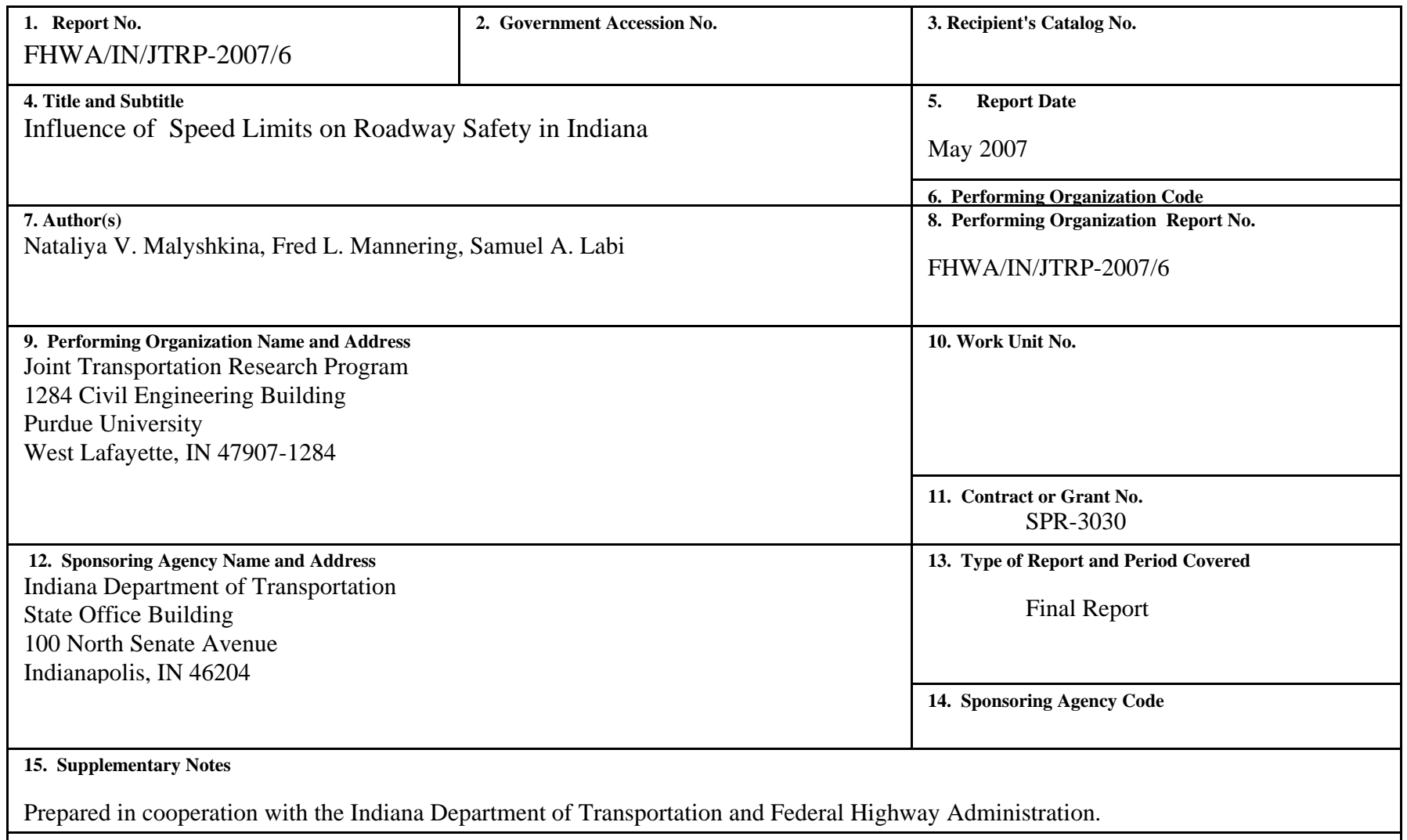

\section{Abstract}

The influence of speed limits on roadway safety is an extremely important social issue and is subject to an extensive debate in the State of Indiana and nationwide. With roughly 900 fatalities and sixty thousand injuries annually in Indiana, traffic accidents place an incredible social and economic burden on the state. Still, speed limits posted on highways and other roads are routinely exceeded as individual drivers try to balance safety and mobility (speed) as well as risks of penalties from enforcement efforts. This research explores the relationship between speed limits and roadway safety. Specifically, the research focuses on the influence of the posted speed limit on the causation of accidents (the likelihood that unsafe speed would be listed as the primary cause of the accident), the severity of accidents, and speed-limit compliance with an emphasis on speed-limit changes from $65 \mathrm{mph}$ to $70 \mathrm{mph}$ on rural interstates and from $55 \mathrm{mph}$ to 60 mph on select non-freeway multilane rural highways. Data were considered on individual accidents from the Indiana Electronic Vehicle Crash Record System in 2004 and 2006. Data from 2005 were excluded because Indiana raised some interstate and non-interstate speeds in July 1, 2005 - thus making 2005 a transition year as drivers adjusted to new speed limits. With these data, appropriate statistical models were estimated for the causation and severity of different types of accidents on all road classes. The results of the modeling show that higher speed limits did not have a statistically significant effect on the likelihood of unsafe-speed being listed as the primary cause of the accident on interstate highways. For some non-interstate highways, higher speed limits were found to significantly increase the likelihood of unsafe speed being listed as the primary cause of the accident, and for others, the higher speed limits were found to decrease this likelihood. With regard to accident severity, the results show that speed limits did not have a statistically significant effect on the severity of accidents on interstate highways. However, for some non-interstate highways, higher speed limits were found to be associated with higher accident severities - suggesting that future speed limit changes on non-interstates need to be carefully assessed on a case-by case basis. The findings in this project provide some information to the Indiana Department of Transportation on the potential consequences of future speed-limit policies in the state.

17. Key Words
Speed limits, speed-limit compliance, speed limits and safety,
vehicle-accident severity, vehicle-accident causation.

18. Distribution Statement

No restrictions. This document is available to the public through the National Technical Information Service, Springfield, VA 22161

\begin{tabular}{|c|c|c|c|}
\hline 19. Security Classif. (of this report) & 20. Security Classif. (of this page) & 21. No. of Pages & 176 \\
Unclassified & Unclassified & Price \\
\hline
\end{tabular}


TABLE OF CONTENTS

Page

TABLE OF CONTENTS

LIST OF TABLES iii

LIST OF FIGURES

CHAPTER 1. INTRODUCTION 1

CHAPTER 2. METHODOLOGY OF STATISTICAL MODELING 10

CHAPTER 3. DATA DESCRIPTION 17

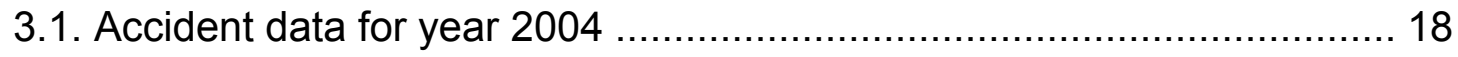

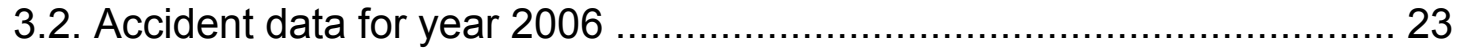

CHAPTER 4. ACCIDENT CAUSATION STUDY 27

4.1. Modeling Procedures: accident causation ........................................ 27

4.2. Results: accident causation models ............................................. 34

4.2.1. Effect of Speed Limit ......................................................... 35

4.2.2. Effect of Other Explanatory Variables ……................................ 40

CHAPTER 5. ACCIDENT SEVERITY STUDY 42

5.1. Modeling Procedures: accident severity ......................................... 42

5.2. Results: accident severity models ................................................. 44

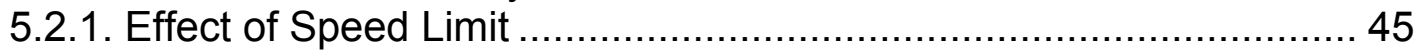

5.2.2. Effect of Other Explanatory Variables ..................................... 51

CHAPTER 6. SPEED LIMITS AND SPEED-LIMIT COMPLIANCE 56

6.1. Effect of Speed Limits on Drivers' Choice of Speed ............................. 56

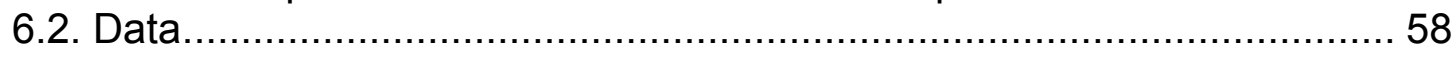

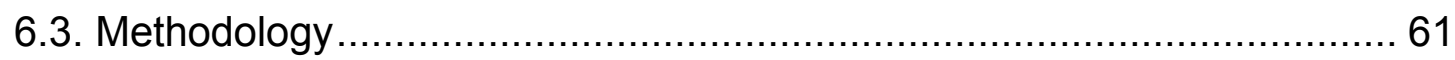

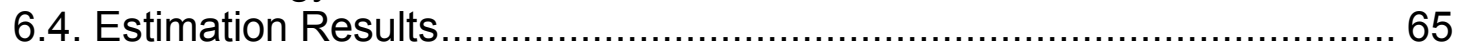

CHAPTER 7. SUMMARY AND CONCLUSIONS 69

LIST OF REFERENCES $\quad 74$

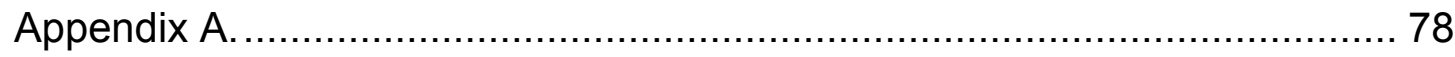

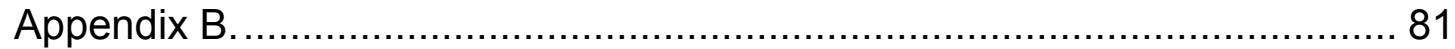

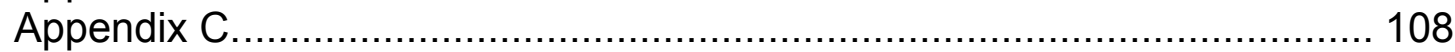




\section{LIST OF TABLES}

Table

Page

Table 4.12004 accident causation models: results for speed limit

Table 4.22006 accident causation models: results for speed limit ..................37

Table 5.12004 accident severity models: results for speed limit ...................47

Table 5.2 2006 accident severity models: results for speed limit ....................48

Table 5.3 Speed limit effect on structure of 2004 accident severity models ......49

Table 5.4 Speed limit effect on structure of 2006 accident severity models ......50

Table 6.1 Survey Questions

Table 6.2 Sample statistics (standard deviation in parentheses when appropriate)

Table 6.3 Statistics for variables found to be significant in model estimation ....65

Table 6.4 Seemingly unrelated regression equation (SURE) estimation results for the number of miles per hour above the speed limit drivers report as their usual speed ( $t$-statistics in parentheses) ${ }^{a}$.... 66

Table B.1 Road classes \& accident types in 2004 accident causation study ....81

Table B.2 Road classes \& accident types in 2006 accident causation study ....82

Table B.3 Binary logit models for 2004 accident causation ............................83

Table B.4 Binary logit models for 2006 accident causation ...........................94

Table B.5 Tests of car-SUV separation in 2004 accident causation study ......106

Table B.6 Tests of car-SUV separation in 2006 accident causation study ......107

Table C. 1 Road classes and accident types in 2004 accident severity study.. 108

Table C. 2 Road classes and accident types in 2006 accident severity study.. 109

Table C. 3 Speed limit data bins chosen in 2004 accident severity study ........110

Table C.4 Speed limit data bins chosen in 2006 accident severity study ........111

Table C.5 Multinomial logit models for 2004 accident severity......................112

Table C.6 Multinomial logit models for 2006 accident severity......................142

Table C.7 Tests of car-SUV separation in 2004 accident severity study .........169

Table C.8 Tests of car-SUV separation in 2006 accident severity study .........170 


\section{LIST OF FIGURES}

Figure

Figure 3.1 Percentage distribution of 2004 accidents by road class

Figure 3.2 Percentage distribution of 2004 accidents by their type

Figure 3.3 Percentage distribution of 2004 accidents by their causation ..........20

Figure 3.4 Percentage distribution of 2004 accidents by their severity level .....21

Figure 3.5 Percentage distributions of 2004 accidents by their causation in four

different speed limit data bins

Figure 3.6 Percentage distributions of 2004 accidents by their severity level in four different speed limit data bins 22

Figure 3.7 Percentage distribution of 2006 accidents by road class ................23

Figure 3.8 Percentage distribution of 2006 accidents by their type .................24

Figure 3.9 Percentage distribution of 2006 accidents by their causation ..........24

Figure 3.10 Percentage distribution of 2006 accidents by their severity level ...25

Figure 3.11 Percentage distributions of 2006 accidents by their causation in four

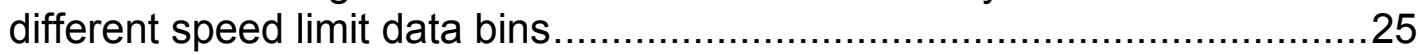

Figure 3.12 Percentage distributions of 2006 accidents by their severity level in four different speed limit data bins .....................................................26

Figure 4.1 Data division by road class and by accident type ...........................29

Figure 4.2 Model estimation procedures .................................................. 31

Figure 4.3 T-ratios of statistically significant speed limit coefficients in 2004

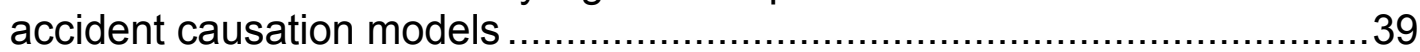

Figure 4.4 T-ratios of statistically significant speed limit coefficients in 2006 accident causation models

Figure 6.1 The effect of Speed Limits on Driving Speed 


\section{CHAPTER 1. INTRODUCTION}

Speed-limit changes that took effect on July 1,2005 , made Indiana the $30^{\text {th }}$ U.S. state to raise rural interstate speed limits up to $70 \mathrm{mph}$ (for vehicles under 13 tons). The top speed limit value was increased on some portions of the state's interstate highway system from $65 \mathrm{mph}$ to $70 \mathrm{mph}$. Speed limits were also increased on some non-interstate, non-freeway rural multilane highways from $55 \mathrm{mph}$ to $60 \mathrm{mph}$. This increase intensified an important debate in the engineering community on the tradeoff between highway mobility (speed) and safety. On one hand, as speed increases, travel times decrease, which reduces transportation costs and leads to an increased productivity and a noticeable positive effect for the national economy. On the other hand, higher speed can possibly have a negative effect on roadway safety.

The relationship between speed limits and roadway safety is not as obvious as it may seem. The reason is that there are several important issues in this relationship. From the basic physics involved, as speed increases, vehicles have higher kinetic energy, travel larger distances during human reaction times, and vehicles are exposed to stronger aerodynamic and centrifugal forces. This tends to increase accident frequency and severity. But there are factors that may work against this. For example, as speed limits increase, the variance of vehicle speeds may decrease, resulting in easier and safer driving conditions. In addition, there are other influences, such as the intensity of police enforcement of posted speed-limit compliance and the engineering design characteristics with relating to roadway design speed. As a result, the overall effect of a speed limit increase on road safety can be ambiguous, and requires 
a thorough study. Such a study and a detailed analysis of the relationship between speed limits and safety on Indiana roads will be undertaken in this project.

In general, there are two measures of road safety that are commonly considered:

1. The first measure evaluates accident frequencies on roadway sections. The accident frequency on a road section is obtained by counting the number of accidents occurring on this section during a specified period of time. Then count-data statistical models (e.g. Poisson, negative binomial models and their zero-inflated counterparts) are typically estimated for accident frequencies on different road sections. The explanatory variables used in these models are the road section characteristics (e.g. road section length, curvature, slope, type, etc).

2. The second measure evaluates accident severity outcomes as determined by the injury level sustained by the most severely injured individual (if any) involved into the accident. This evaluation is done by using data on individual accidents and estimating discrete outcome statistical models (e.g. ordered probit and multinomial logit models) for the accident severity outcomes. The explanatory variables used in these models are the individual accident characteristics (e.g. time and location of an accident, weather conditions and road characteristics at the accident location, characteristics of the vehicles and drivers involved, etc).

These two measures of read safety are complementary - an accident frequency study gives a statistical model of the probability of an accident occurring on a road section and an accident severity study gives a statistical model of the conditional probability of a severity outcome of an accident, given an accident has occurred. 
There are a considerable number of road-safety studies that consider one or both of the two road safety measures described above. Some of the key studies include the following:

- Shankar et al. (1996) used a nested logit model for statistical analysis of accident severity outcomes on rural highways in Washington State. They found that environment conditions, highway design, accident type, driver and vehicle characteristics significantly influence accident severity. They found that overturn accidents, rear-end accidents on wet pavement, fixed-object accidents, and failures to use the restraint belt system lead to higher probabilities of injury or/and fatality accident outcomes, while icy pavement and single-vehicle collisions lead to higher probability of property damage only outcomes.

- Shankar et al. (1997) studied the distinction between safe and unsafe road sections by estimating zero-inflated Poisson and zero-inflated negative binomial models for accident frequencies in Washington State (for these models the zero state corresponds to near zero accident likelihood on safe road sections).

- Duncan et al. (1998) applied an ordered probit model to injury severity outcomes in truck-passenger car rear-end collisions in North Carolina. They found that injury severity is increased by darkness, high speed differentials, high speed limits, wet grades, drunk driving, and being female.

- Karlaftis and Tarko (1998) considered heterogeneous panel data for frequencies of accidents occurred in Indiana over a 6-year period. They developed an improved method of accident frequency modeling in panel data, which is based on a two-step approach: first, heterogeneous data is divided into homogeneous groups by determining (dis)similarities and using cluster analysis; second, negative binomial models are estimated separately for each homogeneous data group. The results obtained by Karlaftis and Tarko clearly indicate that there are significant differences 
between the accident frequency models estimated for urban, suburban and rural counties.

- Chang and Mannering (1999) focused on the effects of trucks and vehicle occupancies on accident severities. They estimated nested logit models for severity outcomes of truck-involved and non-truck-involved accidents in Washington State and found that accident injury severity is noticeably worsened if the accident has a truck involved, and that the effects of trucks are more significant for multi-occupant vehicles than for singleoccupant vehicles.

- Carson and Mannering (2001) studied the effect of ice warning signs on ice-accident frequencies and severities in Washington State. They modeled accident frequencies and severities by using zero-inflated negative binomial and logit models respectively. They found that the presence of ice warning signs was not a significant factor in reducing iceaccident frequencies and severities.

- Khattak (2001) estimated ordered probit models for severity outcomes of multi-vehicle rear-end accidents in North Carolina. In particular, the results of his research indicate that in two-vehicle collisions the leading driver is more likely to be severely injured, in three-vehicle collisions the driver in the middle is more likely to be severely injured, and being in a newer vehicle protects the driver in rear-end collisions.

- Ulfarsson (2001) and Ulfarsson and Mannering (2004) focused on male and female differences in analysis of accident severity. They used multinomial logit models and accident data from Washington State. They found significant behavioral and physiological differences between genders, and also found that probability of fatal and disabling injuries is higher for females as compared to males.

- Kockelman and Kweon (2002) applied ordered probit models to modeling of driver injury severity outcomes. They used a nationwide accident data 
sample and found that pickups and sport utility vehicles are less (more) safe than passenger cars in single-vehicle (two-vehicle) collisions.

- Lee and Mannering (2002) estimated zero-inflated count-data models and nested logit models for frequencies and severities of run-off-roadway accidents in Washington State. They found that run-off-roadway accident frequencies can be reduced by avoiding cut side slopes, decreasing (increasing) the distance from outside shoulder edge to guardrail (light poles), and decreasing the number of isolated trees along roadway. The results of their research also show that run-off-roadway accident severity is increased by alcohol impaired driving, high speeds, and the presence of a guardrail.

- Abdel-Aty (2003) used ordered probit models for analysis of driver injury severity outcomes at different road locations (roadway sections, signalized intersections, toll plazas) in Central Florida. He found higher probabilities of severe accident outcomes for older drivers, male drivers, those not wearing seat belt, drivers who speed, those who drive vehicles struck at the driver's side, those who drive in rural areas, and drivers using electronic toll collection device (E-Pass) at toll plazas.

- Kweon and Kockelman (2003) studied probabilities of accidents and accident severity outcomes for a given fixed driver exposure (which is defined as the total miles driven). They used Poisson and ordered probit models, and considered a nationwide accident data sample. After normalizing accident rates by driver exposure, the results of their study indicate that young drivers are far more crash prone than other drivers, and that sport utility vehicles and pickups are more likely to be involved in rollover accidents.

- Yamamoto and Shankar (2004) applied bivariate ordered probit models to an analysis of driver's and passenger's injury severities in collisions with fixed objects. They considered a 4-year accident data sample from Washington State and found that collisions with leading ends of guardrail 
and trees tend to cause more severe injuries, while collisions with sign posts, faces of guardrail, concrete barrier or bridge and fences tend to cause less severe injuries. They also found that proper use of vehicle restraint system strongly decreases the probability of severe injuries and fatalities.

- Khorashadi et al. (2005) explored the differences of driver injury severities in rural and urban accidents involving large trucks. Using 4years of California accident data and multinomial logit model approach, they found considerable differences between rural and urban accident injury severities. In particular, they found that the probability of severe/fatal injury increases by $26 \%$ in rural areas and by $700 \%$ in urban areas when a tractor-trailer combination is involved, as opposed to a single-unit truck being involved. They also found that in accidents where alcohol or drug use is identified, the probability of severe/fatal injury is increased by $250 \%$ and $800 \%$ in rural and urban areas respectively.

- Islam and Mannering (2006) studied driver aging and its effect on male and female single-vehicle accident injuries in Indiana. They employed multinomial logit models and found significant differences between different genders and age groups. Specifically, they found an increase in probabilities of fatality for young and middle-aged male drivers when they have passengers, an increase in probabilities of injury for middle-aged female drivers in vehicles 6 years old or older, and an increase in fatality probabilities for males older than 65 years old.

- Savolainen (2006), Savolainen and Mannering (2006a) and Savolainen and Mannering (2006b) focused on an important topic of motorcycle safety on Indiana roads. He used multinomial and nested logit models and found that poor visibility, unsafe speed, alcohol use, not wearing a helmet, right-angle and head-on collisions, and collisions with fixed objects cause more severe motorcycle-involved accidents. 
As far, as the relationship between speed and road safety is concerned two general findings have emerged. First, on all road classes (urban streets, highways, etc) vehicle operating speeds tend to exceed the posted speed limit (Renski et al., 1999; Khan, 2002). Second, there are no sure indications that a reasonable increase in speed limit has a considerable negative impact on traffic safety. For example, O'Donnell and Connor (1996) estimated logit and probit models for injury severity outcomes of accidents in Australia and determined that effects of an increase in vehicle speed from 42 to 100 kilometers per hour (from $26.1 \mathrm{mph}$ to $62.1 \mathrm{mph}$ ) are surprisingly small. Shankar et al. (1997) used zero-inflated Poisson and zero-inflated negative binomial models for a study of accident frequencies. They found that a speed limit increase reduced accident frequencies on road sections in the Western part of Washington State, and had no statistically significant effect on accident frequencies in the Eastern part. Very similar results were obtained by Milton and Mannering (1998), who estimated negative binomial models for frequencies of accidents on sections of principal arterials in Washington State in 1992 and 1993 and found a reduction of the frequencies with a speed limit increase. Renski et al. (1999) specifically addressed the effect of speed limit on injury severity outcomes in single-vehicle accidents on interstate highways in North Carolina. They used a pairedcomparison analysis and ordered probit modeling. They found that while increasing speed limits from 55 to $60 \mathrm{mph}$ and from 55 to $65 \mathrm{mph}$ increased the probability of sustaining minor and non-incapacitating injuries, increasing speed limits from 65 to $70 \mathrm{mph}$ did not have a significant effect on accident severity. An analysis of speed limit policies for Indiana was recently carried out by Khan (2002). He found that while previous upward changes in speed limits in Indiana during the past two decades did increase speeds observed on roads, there was no statistically significant evidence to indicate that such increases had a negative impact on safety. 
In the present study we focus on the relationship between the posted speed limit and road safety. We consider data on individual accidents and use statistical and econometric methodologies to analyze three discrete outcomes. In doing so, our study differs from the previous studies in the analysis of vehicle accident data in that we study both the severity and causation of accidents. We use as a basis for our statistical modeling data compiled from Indiana accident databases for different types of accidents (single-vehicle accidents, car or SUV versus truck accidents, etc) and for all classes of roads (interstate highways, urban streets, US routes, etc).

To statistically assess the effect that speed limits have on roadway safety, we will study the following:

1. The relationship between speed limits and the primary cause of the accident. The idea here is to study a relationship between the posted speed limit and the probability of unsafe and/or excessive speed being the primary cause of the accident by the officer at the scene of the accident. This is be done by estimating of appropriate statistical models for the unsafe-speed-related accident causation. However, considerable caution must be exercised when interpreting the results of this analysis because the determination of the officer at the accident scene as to what constitutes the primary cause is an opinion that may be influenced by a multitude of factors. The consistency of these responses across officers and accidents is likely to be questionable. Still, we provide the analysis with this caveat in mind.

2. The relationship between speed limits and the injury severity of the accident. For this we will estimate statistical models for the level of accident severity (determined by the injury level sustained by the most critically injured individual in the accident) which are defined as three discrete outcomes: no-injury, injury and fatality. We will statistically test whether the posted speed limit has any effect on accident severity. 


\section{Factors that determine the effect that speed limits have on drivers'} speed. We will study the self reported speeding behavior of drivers on interstate highways by looking at the speed they normally drive on interstate highways with posted speed limits of $55 \mathrm{mph}, 65 \mathrm{mph}$ and 70 $\mathrm{mph}$. Although there are obvious limitations in using self-reported data, the statistical assessment of these data will still provide some insights even with these limitations in mind.

To understand the effect of speed limits on safety, while modeling accident causation and severity, we will control for other possible confounding effects, such as road characteristics, weather conditions, driver characteristics, and so on. To increase the predictive power of our models, we will consider accidents separately for each combination of accident type and road class (e.g. singlevehicle accidents on urban streets will be considered separately from car-truck accidents on interstate highways). The use of the above two accident modeling approaches will provide important new insights and sufficient statistical evidence on the effect of the posted speed limit on roadway safety. And, statistically modeling self-reported speed limit compliance will provide some insight into how driver behavior may be changing in response to increased speed limits. 


\section{CHAPTER 2. METHODOLOGY OF STATISTICAL MODELING}

Our study deals with accident causation and accident severity, both are nonquantitative discrete outcomes of traffic accidents. The most widely used statistical models for non-count data that is composed of discrete outcomes are the multinomial logit model and the ordered probit model. However, there are two potential problems with applying ordered probability models to accident severity outcomes (Savolainen and Mannering 2006b). The first is related to the fact that non-injury accidents are likely to be under-reported in accident data because they are less likely to be reported to authorities. The presence of under-reporting in an ordered probability model can result in biased and inconsistent model coefficient estimates. In contrast, the coefficient estimates of an unordered multinomial logit probability model are consistent except for the constant terms (Washington et. al. 2003, page 279). The second problem is related to undesirable restrictions that ordered probability models place on influences of the explanatory variables (Washington et. al. 2003, page 294). As a result, in our research study we use and estimate binary and multinomial logit models for accident causation and severity.

The multinomial logit model can be introduced as follows. Let there be $N$ available data observations and $I$ possible discrete outcomes in each observation. Then in the multinomial logit model the probability $P_{n}^{(i)}$ of the $i^{\text {th }}$ outcome in the $n^{\text {th }}$ observation is specified by equation (Washington et al., 2003, page 263)

$$
P_{n}^{(i)}=\frac{\exp \left(\boldsymbol{\beta}_{\mathbf{i}}^{\prime} \mathbf{X}_{\mathbf{i n}}\right)}{\sum_{j=1}^{I} \exp \left(\boldsymbol{\beta}_{\mathbf{j}}^{\prime} \mathbf{X}_{\mathbf{j n}}\right)}, \quad i=1,2,3, \ldots, I, \quad n=1,2,3, \ldots, N . \quad \text { Eq. } 2.1
$$


Here $\mathbf{X}_{\mathrm{in}}$ is the vector of explanatory variables for the $n^{\text {th }}$ observation and $\boldsymbol{\beta}_{\mathbf{i}}$ is the vector of model coefficients to be estimated ( $\boldsymbol{\beta}_{\mathrm{i}}^{\prime}$ is the transpose of $\boldsymbol{\beta}_{\mathrm{i}}$ ). We use a conventional assumption that the first component of vector $\mathbf{X}_{\text {in }}$ is equal to unity, and therefore, the first component of vector $\boldsymbol{\beta}_{\mathbf{i}}$ is the intercept in linear product $\boldsymbol{\beta}_{\mathrm{i}}^{\prime} \mathbf{X}_{\text {in }}$. Note that $P_{n}^{(i)}$, given by Equation (2.1), is a valid probability set for $I$ discrete outcomes because the necessary and sufficient conditions $P_{n}^{(i)} \geq 0$ and $\sum_{i=1}^{I} P_{n}^{(i)}=1$ are obviously satisfied ${ }^{1}$.

We can multiply the numerator and denominator of the fraction in Equation (2.1) by an arbitrary number without any change of the probabilities. As a result, without any loss of generality we can set one of the intercepts to zero. We choose the first component of vector $\boldsymbol{\beta}_{I}$ to be zero in this case. Moreover, if the vector of explanatory variables does not depend on discrete outcomes, i.e. if $\mathbf{X}_{\mathrm{in}} \equiv \mathbf{X}_{\mathbf{n}}$, then without any loss of generality we can set one of vectors of model coefficients to zero. We choose vector $\boldsymbol{\beta}_{I}$ to be zero in this case.

Because accidents are independent events, the likelihood function $L$ and the log-likelihood function $L L$ for the set of probabilities given in Equation (2.1) are obviously equal to

$$
L=\prod_{n=1}^{N} \prod_{i=1}^{I}\left[P_{n}^{(i)}\right]^{i n}, \quad L L=\sum_{n=1}^{N} \sum_{i=1}^{I} \delta_{i n} P_{n}^{(i)},
$$

where $\delta_{\text {in }}$ is defined to be equal to unity if the $i^{\text {th }}$ discrete outcome is observed in the $n^{\text {th }}$ observation and to zero otherwise.

${ }^{1}$ Equation (2.1) can formally be derived by using a linear specification $U_{\text {in }} \equiv \boldsymbol{\beta}_{\mathbf{i}}^{\prime} \mathbf{X}_{\text {in }}+\widetilde{\varepsilon}_{\text {in }}$, by defining $P_{n}^{(i)}=\operatorname{Prob}\left\{U_{i n} \geq \max _{\forall j \neq i}\left(U_{j n}\right)\right\}$ and by choosing the Gumbel (Type I) extreme value distribution for the i.i.d. random error terms $\widetilde{\varepsilon}_{i n}$. For details see Washington et al., 2003. 
Now we assume that the explanatory variables vector is independent of the discrete outcomes, $\quad \mathbf{X}_{\mathrm{in}} \equiv \mathbf{X}_{\mathrm{n}}$, and consider two simple special cases of the multinomial logit model. First, if there are just two possible discrete outcomes, $I=2$ and $i=1,2$, then in this case the model becomes a binary logit model, and Equation (2.1) simplifies to

$$
P_{n}^{(1)}=\frac{\exp \left(\boldsymbol{\beta}_{1}^{\prime} \mathbf{X}_{\mathbf{n}}\right)}{\exp \left(\boldsymbol{\beta}_{1}^{\prime} \mathbf{X}_{\mathbf{n}}\right)+1}, \quad P_{n}^{(2)}=\frac{1}{\exp \left(\boldsymbol{\beta}_{1}^{\prime} \mathbf{X}_{\mathbf{n}}\right)+1},
$$

where there is only one coefficient vector $\boldsymbol{\beta}_{1}$ to be estimated. Second, if there are three possible discrete outcomes, $I=3$ and $i=1,2,3$, then in this case Equation (2.1) simplifies to

$$
\begin{aligned}
P_{n}^{(1)} & =\frac{\exp \left(\boldsymbol{\beta}_{1}^{\prime} \mathbf{X}_{\mathbf{n}}\right)}{\exp \left(\boldsymbol{\beta}_{1}^{\prime} \mathbf{X}_{\mathbf{n}}\right)+\exp \left(\boldsymbol{\beta}_{2}^{\prime} \mathbf{X}_{\mathbf{n}}\right)+1}, \\
P_{n}^{(2)} & =\frac{\exp \left(\boldsymbol{\beta}_{2}^{\prime} \mathbf{X}_{\mathbf{n}}\right)}{\exp \left(\boldsymbol{\beta}_{1}^{\prime} \mathbf{X}_{\mathbf{n}}\right)+\exp \left(\boldsymbol{\beta}_{2}^{\prime} \mathbf{X}_{\mathbf{n}}\right)+1}, \\
P_{n}^{(3)} & =\frac{1}{\exp \left(\boldsymbol{\beta}_{1}^{\prime} \mathbf{X}_{\mathbf{n}}\right)+\exp \left(\boldsymbol{\beta}_{2}^{\prime} \mathbf{X}_{\mathbf{n}}\right)+1},
\end{aligned}
$$

where there are two coefficient vectors $\boldsymbol{\beta}_{1}$ and $\boldsymbol{\beta}_{2}$ to be estimated. We will use these special-case logit models in the next two chapters.

It is customary to use the maximum likelihood method to estimate unknown vectors of coefficients $\boldsymbol{\beta}_{\mathrm{i}}$ in the logit models given by Equations (2.1), (2.3) and (2.4). Namely, one finds such values of the unknown coefficients that the likelihood function (and correspondingly the log-likelihood function) given by Equation (2.2) reaches its global maximum. In the present study we use econometric software package LIMDEP for all model estimations by means of the maximum likelihood method ${ }^{2}$. We also use MATLAB software package for initial processing of data.

\footnotetext{
${ }^{2}$ LIMDEP can be found at http://www.limdep.com, we use Version 7.0 in our study.
} 
In the next chapters we will need to compare several estimated models in order to infer if there are statistically significant differences among these models. As a result, here we would like to demonstrate how model comparisons are done by using a likelihood ratio test. Assume that we have divided a data sample into different data bins. The likelihood ratio test uses the model estimated for the whole data sample and the models separately estimated for each data bin. The test statistic is (Washington et al., 2003, page 244)

$$
-2\left[L L(\boldsymbol{\beta})-\sum_{m=1}^{M} L L\left(\boldsymbol{\beta}_{\mathrm{m}}\right)\right] \sim \chi_{\mathrm{df}=(M-1) \times K}^{2}, \quad \text { Eq. } 2.5
$$

where $L L(\boldsymbol{\beta})$ is the log-likelihood of the model estimated for the whole data sample and $\boldsymbol{\beta}$ is the vector of coefficients estimated for this model; $L L\left(\boldsymbol{\beta}_{\mathrm{m}}\right)$ is the log-likelihood of the model estimated for observations in the $\mathrm{m}^{\text {th }}$ data bin and $\boldsymbol{\beta}_{\mathrm{m}}$ is the vector of coefficients estimated for this model $(m=1,2,3, \ldots, M) ; M$ is the number of the data bins; $K$ is the number of coefficients estimated for each model (i.e. $K$ is the length of vectors $\boldsymbol{\beta}$ and $\left.\boldsymbol{\beta}_{\mathrm{m}}\right)^{3} ; \chi_{\mathrm{df}=(M-1) \times K}^{2}$ is the chisquared distribution with $(M-1) \times K$ degrees of freedom (df). The zerohypothesis for the test statistic given by Equation (2.5) is that the model estimated for the whole data sample and the combination of the $M$ models separately estimated for the data bins, are statistically the same. In other words, for a chosen confidence level $\pi$ if the left-hand-side of Equation (2.5) is between zero and the $(1-\pi)^{\text {th }}$ percentile of the chi-squared distribution given on the right-hand-side, then we conclude that the division of the data into different bins makes no statistically significant difference for the model estimation. We conclude that there is a difference otherwise.

\footnotetext{
${ }^{3}$ Note that the left-hand-side of Equation (2.5) is always non-negative because a combination of models separately estimated for data bins always provides a fit which is at least as good as the fit for the whole data sample.
} 
At the end of this chapter we describe how the magnitude of the influence of specific explanatory variables on the discrete outcome probabilities can be measured. This is done by elasticity computations (Washington et al., 2003, page 271). Elasticities $E_{X_{j n, k}^{(i)}}^{P_{n}^{(i)}}$ are computed from the partial derivatives of the outcome probabilities for the $n^{\text {th }}$ observation as

$$
E_{X_{j n, k}^{(i)}}^{P_{n}^{(i)}}=\frac{\partial P_{n}^{(i)}}{\partial X_{j n, k}} \cdot \frac{X_{j n, k}}{P_{n}^{(i)}}, \quad i, j=1, \ldots, I, \quad n=1, \ldots, N, \quad k=1, \ldots, K . \quad \text { Eq. } 2.6
$$

Here $P_{n}^{(i)}$ is the probability of outcome $i$ given by Equation (2.1), $X_{j n, k}$ is the $k^{\text {th }}$ component of the vector of explanatory variables $\mathbf{X}_{\mathrm{jn}}$ that enters the formula for the probability of outcome $j$, and $K$ is the length of this vector. If $j=i$, then the elasticity given by Equation (2.6) is called direct elasticity, otherwise, if $j \neq i$, then the elasticity is called cross elasticity. The direct elasticity of the outcome probability $P_{n}^{(i)}$ with respect to variable $X_{i n, k}$ measures the percent change in $P_{n}^{(i)}$ that results from an infinitesimal percentage change in $X_{i n, k}$. Note that $X_{i n, k}$ directly enters the numerator of the formula for $P_{n}^{(i)}$, as given by Equation (2.1). The cross elasticity of $P_{n}^{(i)}$ with respect to variable $X_{j n, k}$ measures the percent change in $P_{n}^{(i)}$ that results from an infinitesimal percentage change in $X_{j n, k}$. Note that $X_{j n, k}$ enters the numerator of the formula for the probability $P_{n}^{(j)}$ of the outcome $j$, which is different from outcome $i$. Thus, cross elasticities measure indirect effects that arise from the fact that the outcome probabilities must sum to unity, $\sum_{i=1}^{I} P_{n}^{(i)}=1$. If the absolute value of the computed elasticity $E_{X_{j n, k}^{(i)}}^{P_{n}^{(i)}}$ of explanatory variable $X_{j n, k}$ is less than unity, then this variable is said to be inelastic, and the resulting percentage change in the outcome probability $P_{n}^{(i)}$ will be less (in its absolute value) than a percentage change in the variable. Otherwise, the variable is said to be elastic. 
Using Equation (2.1) and calculating the derivatives in Equation (2.6), we obtain the formulas for the direct and cross elasticities of explanatory variables in the multinomial logit model:

$$
\begin{array}{ll}
E_{X_{i n, k}}^{P_{(i)}^{(i)}}=\left[1-P_{n}^{(i)}\right] \cdot \beta_{i, k} X_{i n, k} \quad \text { for direct elasticities; } \\
E_{X_{j n, k}^{P_{i}}}^{P^{(i)}}=-P_{n}^{(j)} \cdot \beta_{j, k} X_{j n, k} \quad \text { for cross elasticities, } j \neq i .
\end{array}
$$

Here $\beta_{i, k}$ is the $k^{\text {th }}$ component of the vector of the model estimable coefficients in the formula for the probability $P_{n}^{(i)}$ of outcome $i$ [refer to Equation (2.1)]. If the explanatory variables vector is independent of the discrete outcomes, $\mathbf{X}_{\mathrm{in}} \equiv \mathbf{X}_{\mathrm{n}}$, then Equations (2.7) stay valid with $X_{i n, k} \equiv X_{j n, k} \equiv X_{n, k}$.

It is customary to report averaged elasticities, which are the elasticities averaged over all observations (i.e. averaged over $n=1,2,3, \ldots, N)$ ). Let us consider the cases of two and three possible discrete outcomes, given by Equations (2.3) and (2.4) respectively, and let us average the elasticities given by Equations 2.7) over all observations. Then we find the averaged direct and cross elasticities. In the case of two discrete outcomes $(i=1,2)$ we obtain

$$
\begin{array}{ll}
\bar{E}_{1 ; X_{k}}^{(1)}=\left\langle E_{X_{1, k}}^{P_{n}^{(1)}}\right\rangle_{n}=\left\langle\left[1-P_{n}^{(1)}\right] \cdot \beta_{1, k} X_{n, k}\right\rangle_{n} & \text { averaged direct elasticity; } \\
\bar{E}_{1 ; X_{k}}^{(2)}=\left\langle E_{X_{1 n, k}}^{P_{n}^{(2)}}\right\rangle_{n}=-\left\langle P_{n}^{(1)} \cdot \beta_{1, k} X_{n, k}\right\rangle_{n} & \text { averaged cross elasticity. }
\end{array}
$$

In the case of three discrete outcomes $(i=1,2,3)$ we obtain

$$
\begin{array}{ll}
\bar{E}_{1 ; X_{k}}^{(1)}=\left\langle E_{X_{1 n, k}}^{P_{n}^{(1)}}\right\rangle_{n}=\left\langle\left[1-P_{n}^{(1)}\right] \cdot \beta_{1, k} X_{n, k}\right\rangle_{n} & \text { averaged direct } \\
\text { elasticities; } & \\
\bar{E}_{2 ; X_{k}}^{(2)}=\left\langle E_{X_{2 n, k}}^{P_{n}^{(2)}}\right\rangle_{n}=\left\langle\left[1-P_{n}^{(2)}\right] \cdot \beta_{2, k} X_{n, k}\right\rangle_{n} & \\
\bar{E}_{1 ; X_{k}}^{(2)}=\bar{E}_{1 ; X_{k}}^{(3)}=\left\langle E_{X_{1 n, k}}^{(2,3)}\right\rangle_{n}=-\left\langle P_{n}^{(1)} \cdot \beta_{1, k} X_{n, k}\right\rangle_{n} & \text { averaged cross } \\
\bar{E}_{2 ; X_{k}}^{(1)}=\bar{E}_{2 ; X_{k}}^{(3)}=\left\langle E_{X_{2 n, k}}^{P_{1}^{(1,3)}}\right\rangle_{n}=-\left\langle P_{n}^{(2)} \cdot \beta_{2, k} X_{n, k}\right\rangle_{n} & \text { elasticities. }
\end{array}
$$

Here brackets $\langle\ldots\rangle_{n}$ means averaging over all observations $n=1,2,3, \ldots, N$. 
All elasticity formulas given above are applicable only when explanatory variable $X_{j n, k}$ used in the outcome probability model is continuous. In the case when $X_{j n, k}$ takes on discrete values, the elasticities given by Equation (2.6) can not be calculated, and they are replaced by pseudo-elasticities (for example, see Washington et al., 2003, page 272). The later are given by the following equation, which is an obvious discrete counterpart of Equation (2.6),

$$
E_{X_{j n, k}^{(i)}}^{P_{n}^{(i)}}=\frac{\Delta P_{n}^{(i)}}{\Delta X_{j n, k}} \cdot \frac{X_{j n, k}}{P_{n}^{(i)}}, \quad i, j=1, \ldots, I, \quad n=1, \ldots, N, \quad k=1, \ldots, K . \quad \text { Eq. } 2.10
$$

Here $\Delta P_{n}^{(i)}$ denotes the resulting discrete change in the probability of outcome $i$ due to discrete change $\Delta X_{j n, k}$ in variable $X_{j n, k}$. We will neither calculate nor use pseudo-elasticities in the present research study. 


\section{CHAPTER 3. DATA DESCRIPTION}

The accident data used in the present study is from the Indiana Electronic Vehicle Crash Record System (EVCRS). The EVCRS was launched in 2004 and includes available information on all accidents investigated by Indiana police starting from January 1, 2003.

The information on accidents included into the EVCRS can be divided into three major categories ${ }^{4}$ :

1. An Environmental Record - it includes information on circumstances related to an accident. For example, weather, roadway and traffic conditions, number of dead and injured people involved, etc.

2. A Vehicle and Driver Record - it includes information on all vehicles involved into an accident and on all drivers of these vehicles. For example, accident contributing factors by each vehicle, type and model of each vehicle, posted speed limit for each vehicle, driver's injury status, driver's age and gender, driver's name and address, etc.

3. Non-driver Individual Record - it includes information on all people who are involved into an accident but are not drivers. This record includes only the name and address of those people, but it does not include any information on their injuries (if any).

\footnotetext{
${ }^{4}$ Note that accident data is subject to missing observations and typos. In addition, there can be misidentification errors on police crash reports due police officers' mistakes and prejudices. We eliminate obvious typos during initial data processing and exclude missing observations, but we do not correct for concealed typos and unobserved misidentification errors. Such correction can be done under the Bayesian statistics and Markov Chain Monte Carlo (MCMC) simulations framework, in which one introduces and estimates auxiliary unobserved state variables that indicate unobserved errors (Tsay, 2002, page 413). This is beyond the scope of our study. We assume that police misidentification errors are sufficiently small not to affect our final results.
} 
In our study we use only information from the first two categories above. These two categories include 127 variables for each accident, which is an abundance of data. However, we do not need to consider all these variables. Indeed, because our study focuses on accident causation and severity, we choose all information and all data variables that can reasonably be related to the subject of our study, and we consider only these variables. For example, we do not consider the name of the road where an accident took place and the license plate numbers of the vehicles involved because we can reasonably expect that these variables do not contribute to the accident cause and severity. The list of all variables that we consider and their explanation is given in Appendix A.

In the present study we use data on 204,382 accidents that occurred in 2004 and 182,922 accidents that occurred in 2006. We do not consider 2005 accidents because in 2005 the top speed limit value was raised on some portions of Indiana interstate highways from 65 to $70 \mathrm{mph}$, and we would like to separate our research results and conclusions from the effects of drivers' adjustment to new speed limit values.

\subsection{Accident data for year 2004}

The percentage distributions of 2004 accidents by road class and by accident type are given in Figure 3.1 and Figure 3.2 respectively ${ }^{5}$.

${ }^{5}$ For convenience, from each of the percentage distribution plot we exclude accidents for which the considered descriptive variable (e.g. road class or accident type) is unknown. 


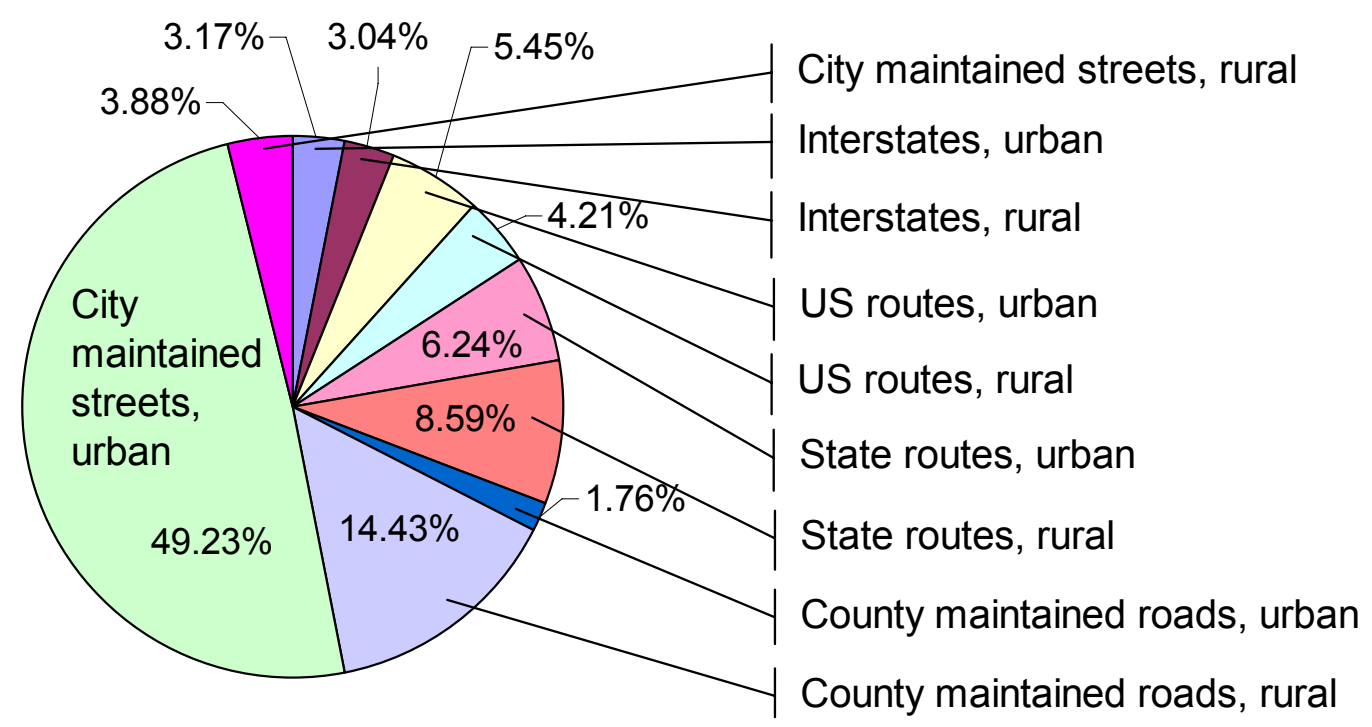

Figure 3.1 Percentage distribution of 2004 accidents by road class

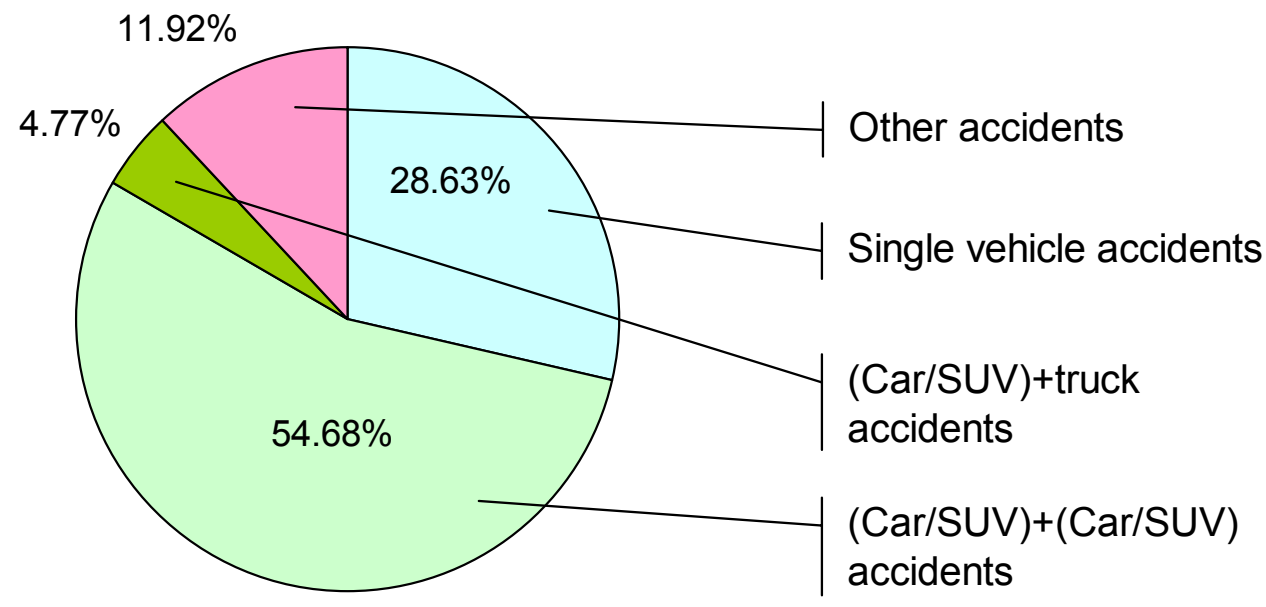

Figure 3.2 Percentage distribution of 2004 accidents by their type 
As stated above, the goal of our study is to analyze the effect of speed limit on unsafe-speed-related causation and severity of accidents. As a result, first, we plot the percentage distributions of all 2004 accidents by their causation and severity level in Figure 3.3 and Figure 3.4 respectively. Second, we divide 2004 accidents into four different speed limit data bins, which respectively include accidents that occurred on roads with low $(\leq 30 \mathrm{mph}$ ), medium-low ( $>30 \mathrm{mph}$ but $\leq 50 \mathrm{mph}$ ), medium-high ( $>50 \mathrm{mph}$ but $\leq 60 \mathrm{mph}$ ) and high ( $>60 \mathrm{mph}$ ) speed limits. Finally, we plot the percentage distributions by accident causation and severity level separately for accidents in each of these chosen speed limit bins. The plots are given in Figure 3.5 and Figure 3.6.

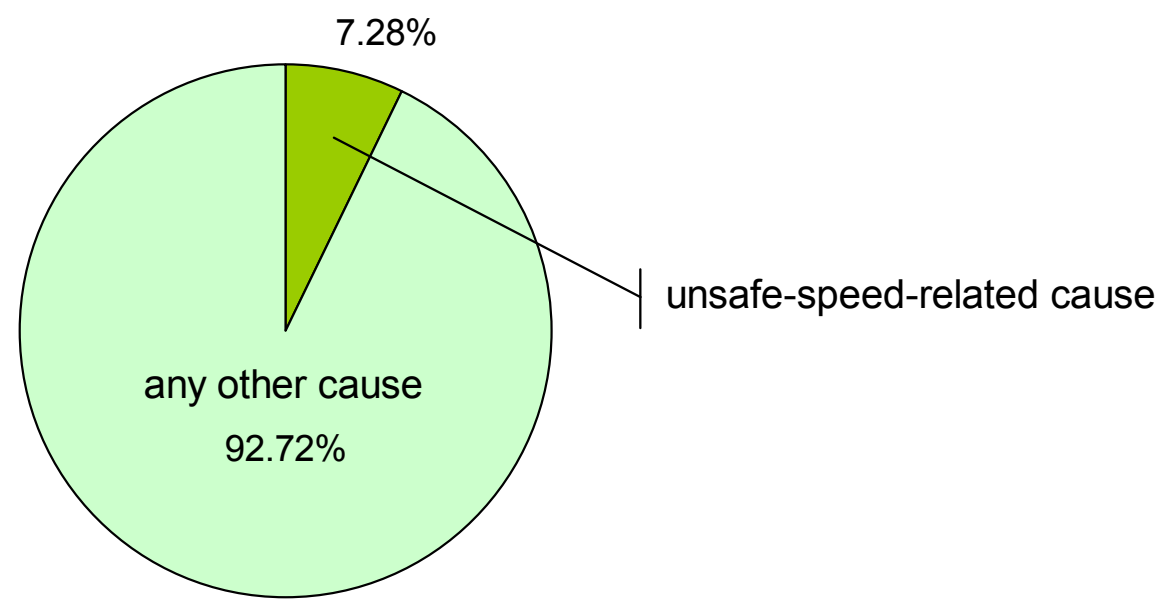

Figure 3.3 Percentage distribution of 2004 accidents by their causation 


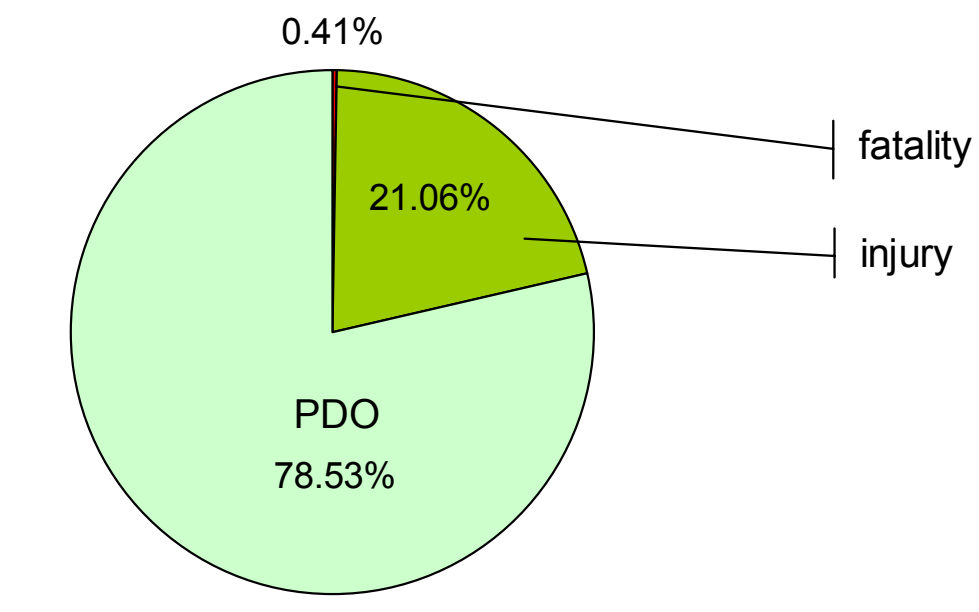

"PDO" means property damage only (no injury)

Figure 3.4 Percentage distribution of 2004 accidents by their severity level

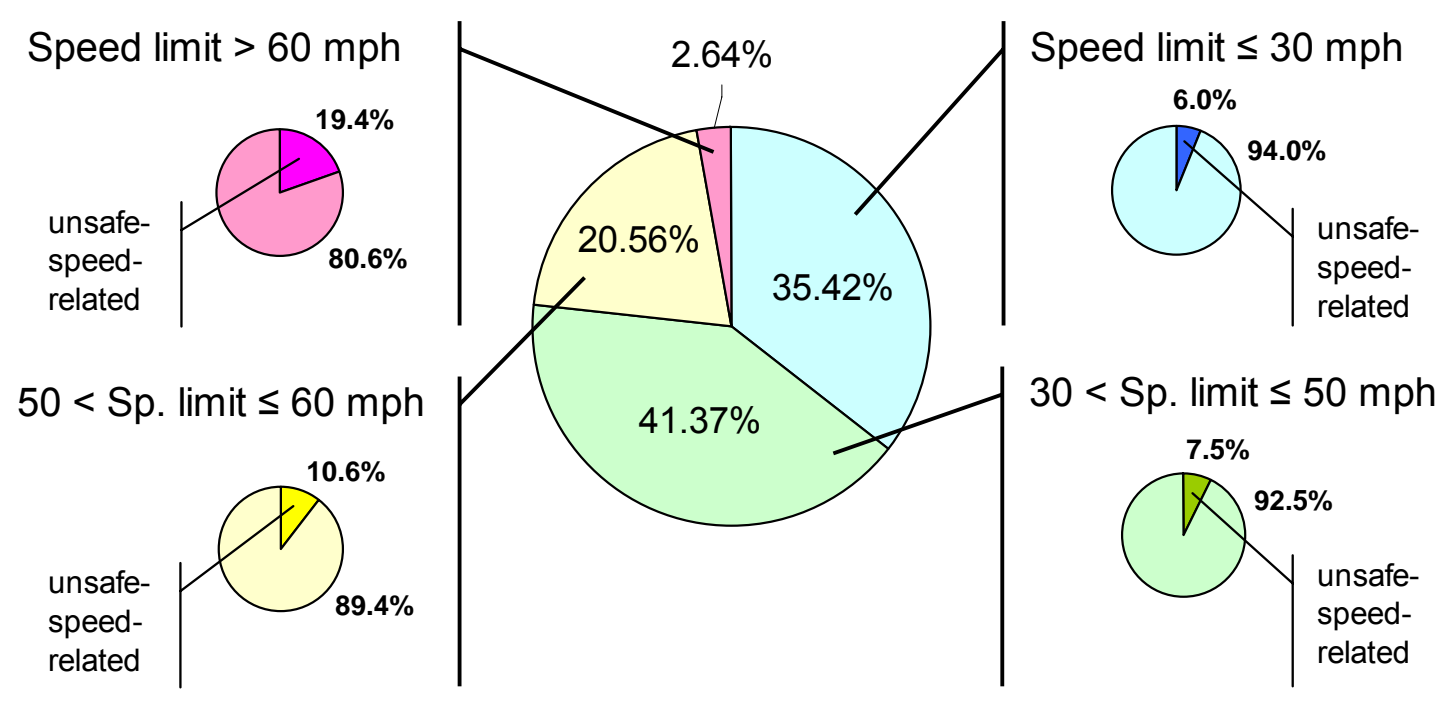

Figure 3.5 Percentage distributions of 2004 accidents by their causation in four different speed limit data bins 


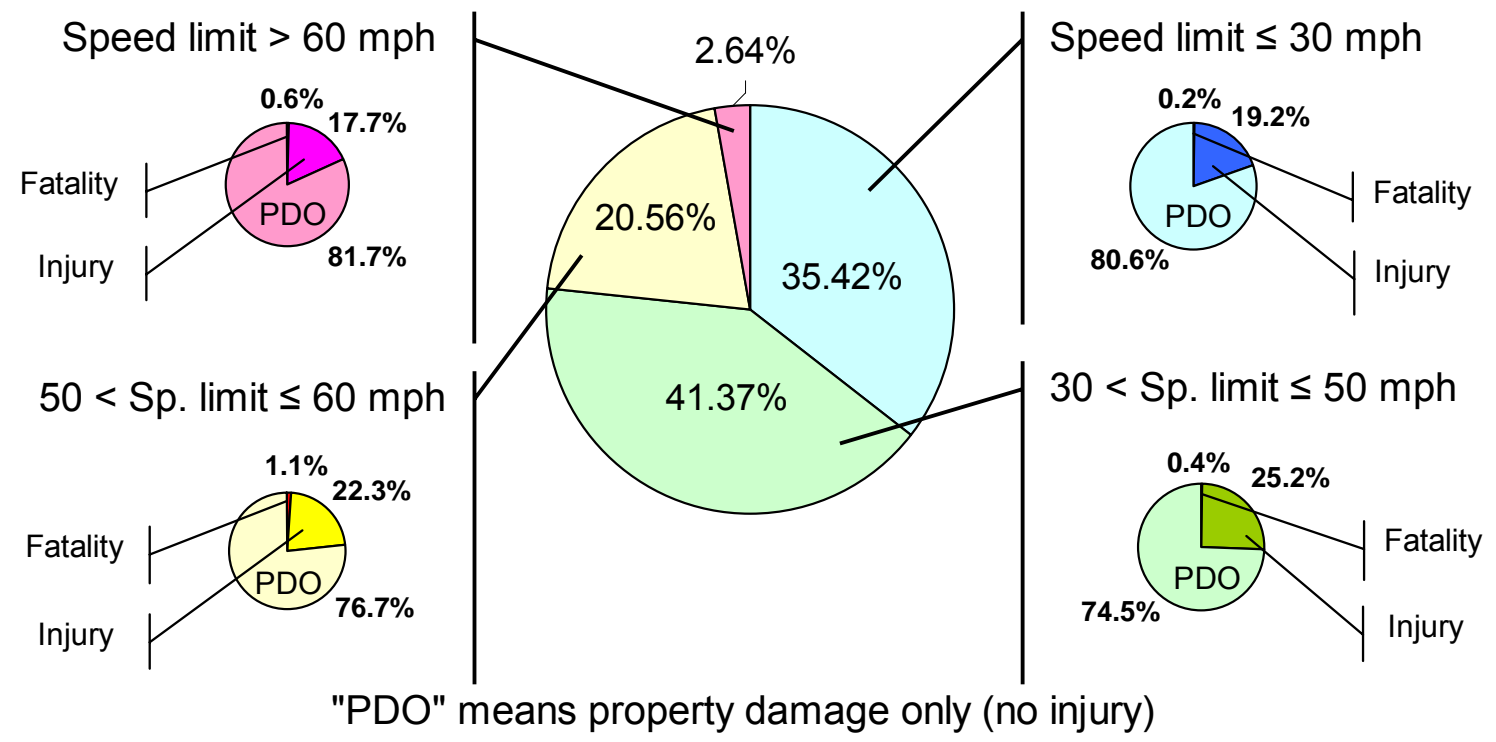

Figure 3.6 Percentage distributions of 2004 accidents by their severity level in four different speed limit data bins

We can make some interesting observations by using the plots in Figure 3.5 and Figure 3.6. First, from Figure 3.5 it seems that the probability of unsafe and/or excessive speed being the primary cause of an accident grows with speed limit. Second, from Figure 3.6 it seems that the posted speed limit does not have a clearly pronounced and easily understandable effect on the severity level of an accident. Indeed, the probabilities of fatality and injury appear to decrease for very high speed limit values ( $>60 \mathrm{mph}$ ). However, we must keep in mind that mathematical relations (or absence of them) inferred from simple descriptive statistics can be spurious. The main reason is that different explanatory variables can be (and usually are) mutually dependent, which greatly complicates the inference problem. Thus, it can well be the case that some other variables impact accident causation and severity, while speed limit simply happens to be correlated with these other variables. As a result, to truly understand the effect of speed limit on accident causation and severity, one has to control for all other relevant variables in making an inference about the effect 
of speed limit. This is done by building appropriate statistical models, and this is the main subject of our research, which is presented in the next two Chapters.

\subsection{Accident data for year 2006}

Now let us describe 2006 accident data that we use. The percentage distributions of 2006 accidents by road class and by accident type are given in Figure 3.7 and Figure 3.8 respectively. The percentage distributions of 2006 accidents by their causation and severity level are plotted in Figure 3.9 and Figure 3.10 respectively. We divide 2006 accidents into four different speed limit data bins the same way as we divided 2004 accidents. The percentage distributions by accident causation and severity level are calculated for 2006 accidents that are inside each of these four speed limit bins and are plotted in Figure 3.11 and Figure 3.12.

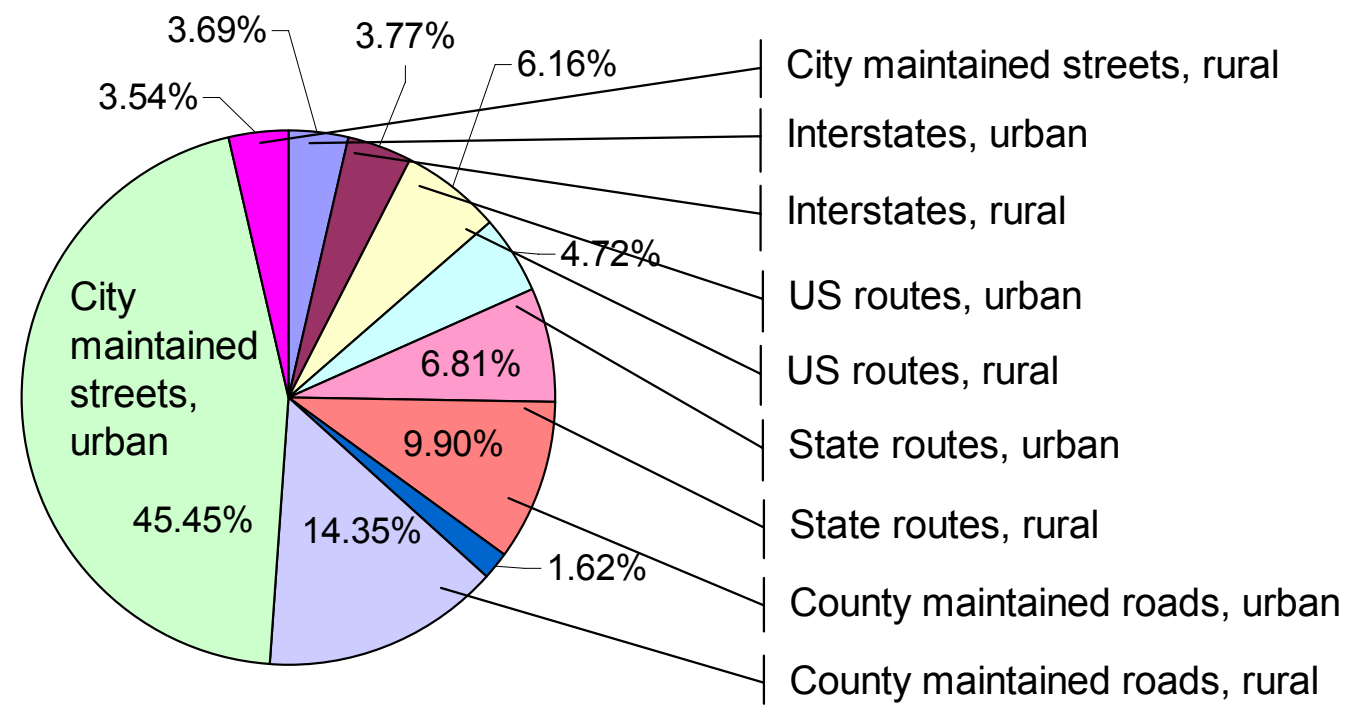

Figure 3.7 Percentage distribution of 2006 accidents by road class 


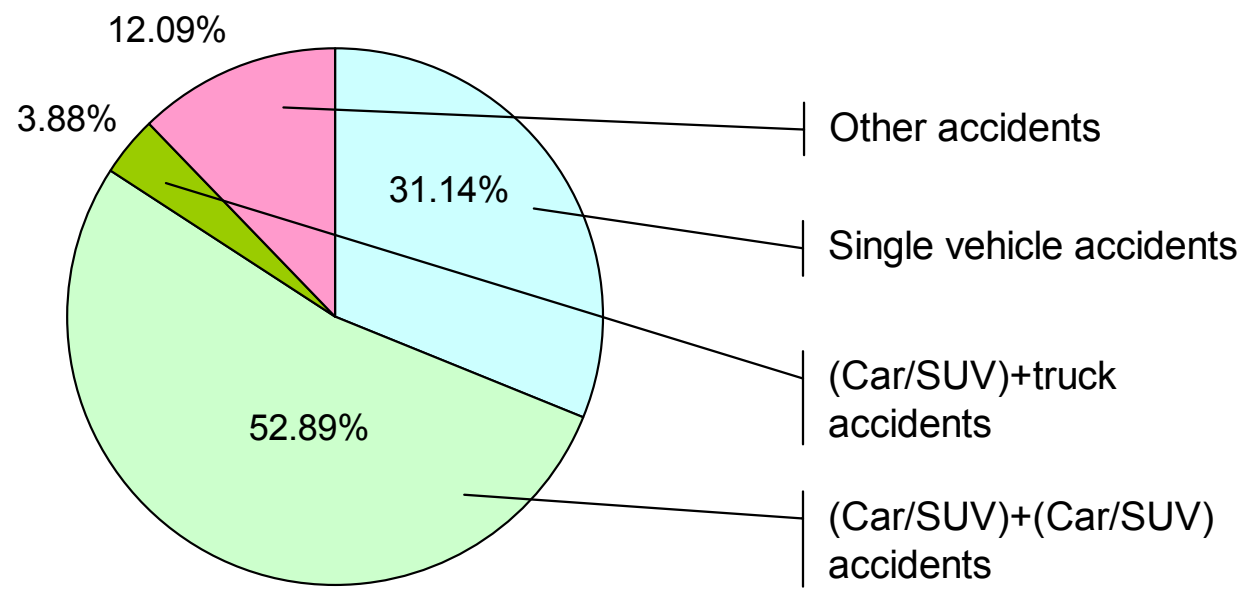

Figure 3.8 Percentage distribution of 2006 accidents by their type

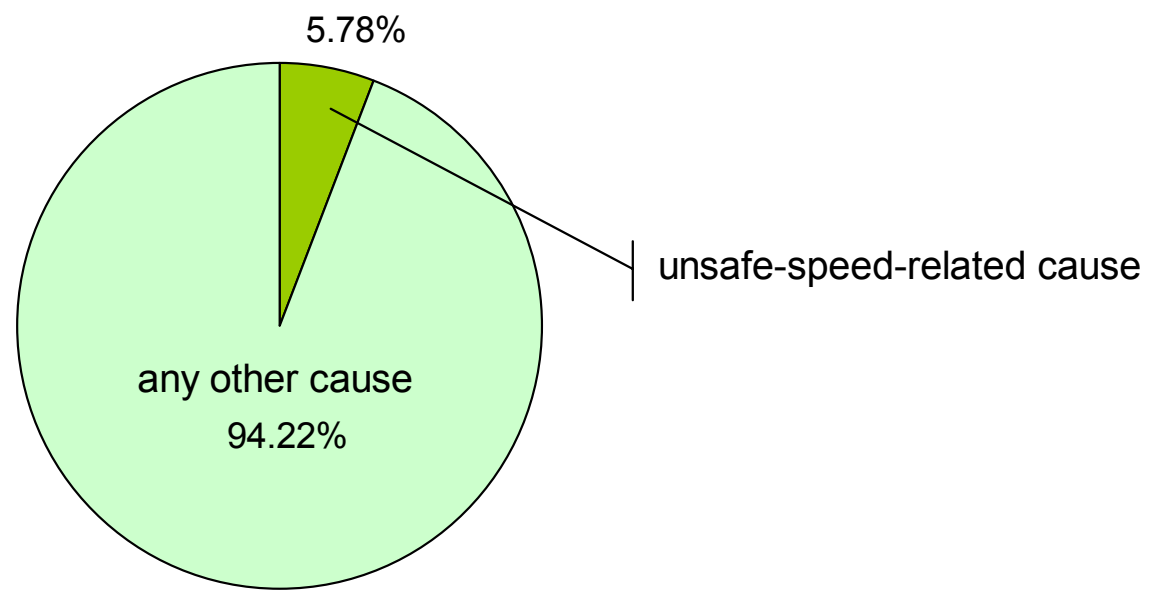

Figure 3.9 Percentage distribution of 2006 accidents by their causation 


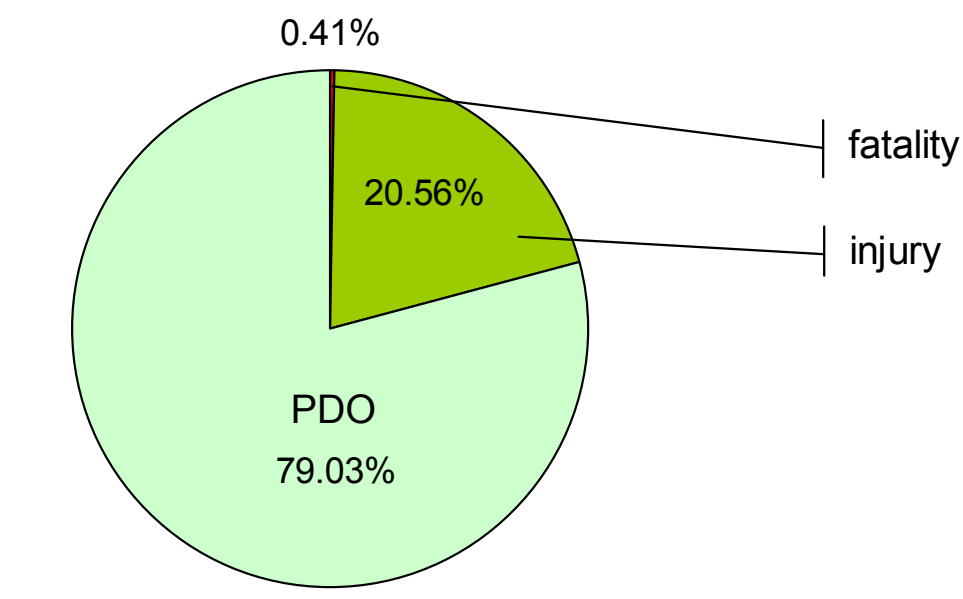

"PDO" means property damage only (no injury)

Figure 3.10 Percentage distribution of 2006 accidents by their severity level

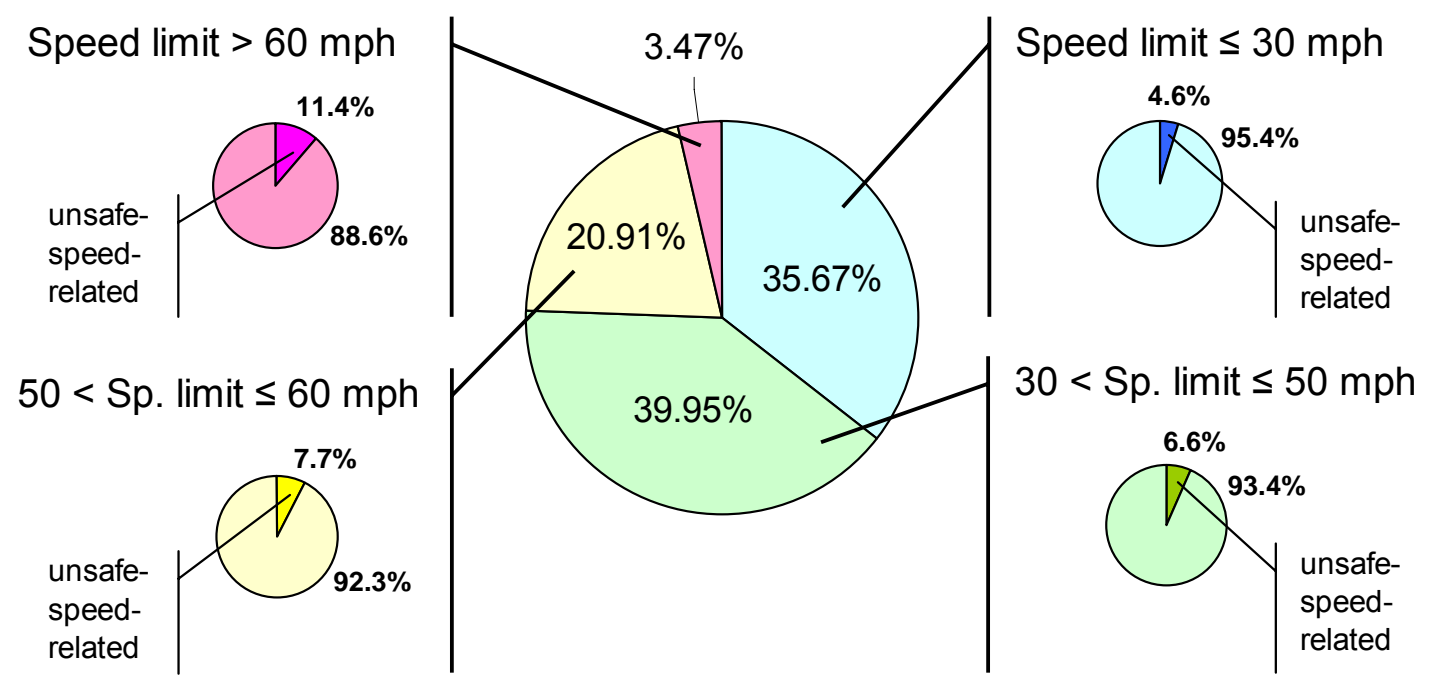

Figure 3.11 Percentage distributions of 2006 accidents by their causation in four different speed limit data bins 


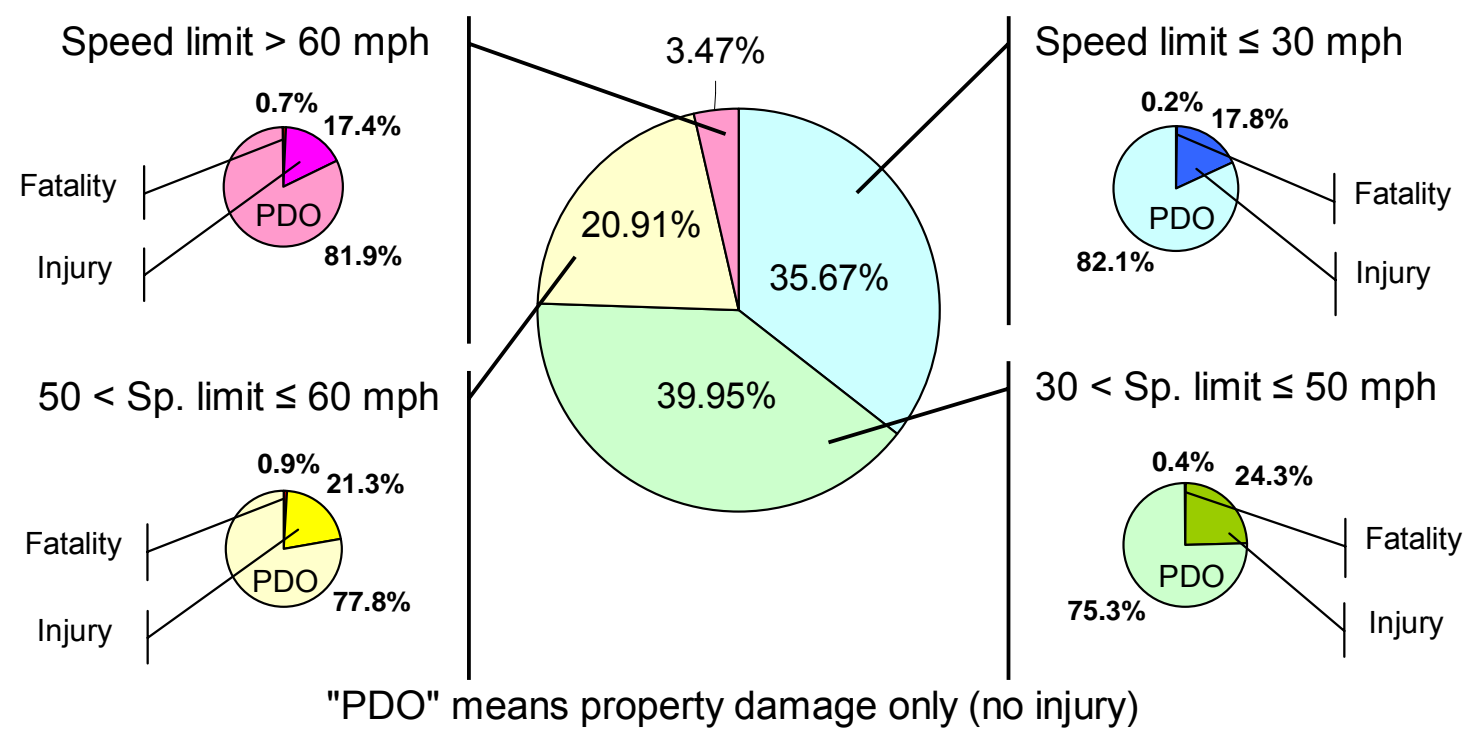

Figure 3.12 Percentage distributions of 2006 accidents by their severity level in four different speed limit data bins

Using the plots in Figure 3.11 and Figure 3.12, we make the same observations for 2006 accidents as those made for 2004 accidents. First, it again seems that the probability of unsafe and/or excessive speed being the primary cause of an accident grows with speed limit (refer to Figure 3.11). Second, from Figure 3.12 it seems that the posted speed limit does not have a clearly pronounced effect on the severity level of an accident because the probabilities of fatality and injury appear to decrease for very high speed limit values (>60 mph). However, we again can not make definite inference on the effect of the speed limit from these observations without building appropriate statistical models for accident causation and severity. 


\section{CHAPTER 4. ACCIDENT CAUSATION STUDY}

In this chapter we study the unsafe-speed-related causation of accidents and its dependence on the posted speed limit and other explanatory variables that characterize accidents. Below, we first explain how we use the available accident data and estimate statistical models for unsafe-speed-related causation. Then, we present the results obtained from the estimation of these models for accidents that happened in Indiana in 2004 and 2006.

\subsection{Modeling Procedures: accident causation}

There exists one primary cause of each accident, as identified by a police officer in his report on this accident ${ }^{6}$. All possible accident primary causes are classified into three categories:

1. Driver-related contributing circumstances (e.g. unsafe speed, speed too fast for weather conditions, driver illness, improper passing, etc.).

2. Vehicle-related contributing circumstances (e.g. tire failure or defective, brake failure or defective, etc.).

3. Environment-related contributing circumstances (e.g. animal on roadway, roadway surface condition, glare, etc.).

Here we are interested in an unsafe and/or excessive speed being the primary cause of an accident and its dependence on the posted speed limit. As a result, we introduce an indicator (dummy) variable that is equal to unity if the primary cause of an accident is either "unsafe speed" or "speed too fast for weather conditions" and is equal to zero for any other primary cause. We then estimate

\footnotetext{
${ }^{6}$ For potential problems with primary cause identification see footnote 4 on page 17.
} 
binary logit models with two possible outcomes that are determined by this indicator variable, refer to equation (2.3).

To uncover the direct influence of the posted speed limit on the accident primary cause, we need to control for other explanatory variables that might also affect accident causation. Examples of these other variables are weather conditions, accident time and date, vehicle and driver characteristics, and so on. All explanatory variables can be divided into two distinct types. First, there are indicator (dummy) variables that are equal to unity if some particular conditions are satisfied, and are equal to zero otherwise. Examples of indicator variables are driver's gender indicator, weekend indicator and precipitation indicator. Second, there are quantitative variables that take on meaningful quantitative values, such as driver's age, speed limit and number of fatalities. In addition, one can easily define derivative indicator variables that are obtained from quantitative variables. For example, one can define a "young driver" indicator as being equal to unity if the driver's age is below 25 . When estimating models, we frequently define and use the most useful (as judged by the model likelihood function) new derivative indicator variables that are based on quantitative variables.

Because results of safety analysis vary significantly across different road classes and accident types (Karlaftis and Tarko, 1998; Chang and Mannering, 1999; Khan, 2002; Kweon and Kockelman, 2003; Ulfarsson and Mannering, 2004; Khorashadi et al., 2005), we divide accident data by road class and accident type as shown in Figure 4.1, and we estimate the accident causation models separately for each road-class-accident-type combination. Note that we do not consider accidents with two trucks involved and with more than two vehicles involved (there are less than $12.1 \%$ of such accidents, see Figure 3.2 and Figure 3.8). For all two-vehicle accident types other than two-truck accidents, we test whether cars and SUVs can be considered together or must 
be considered separately (refer to the additional division shown inside the dotted box in Figure 4.1). This test is done by using the likelihood ratio test, which is explained in CHAPTER 2. The complete list of combinations of different road classes and accident types that we consider in our causation study of 2004 and 2006 accidents can be found in Table B.1 and Table B.2 in Appendix B.

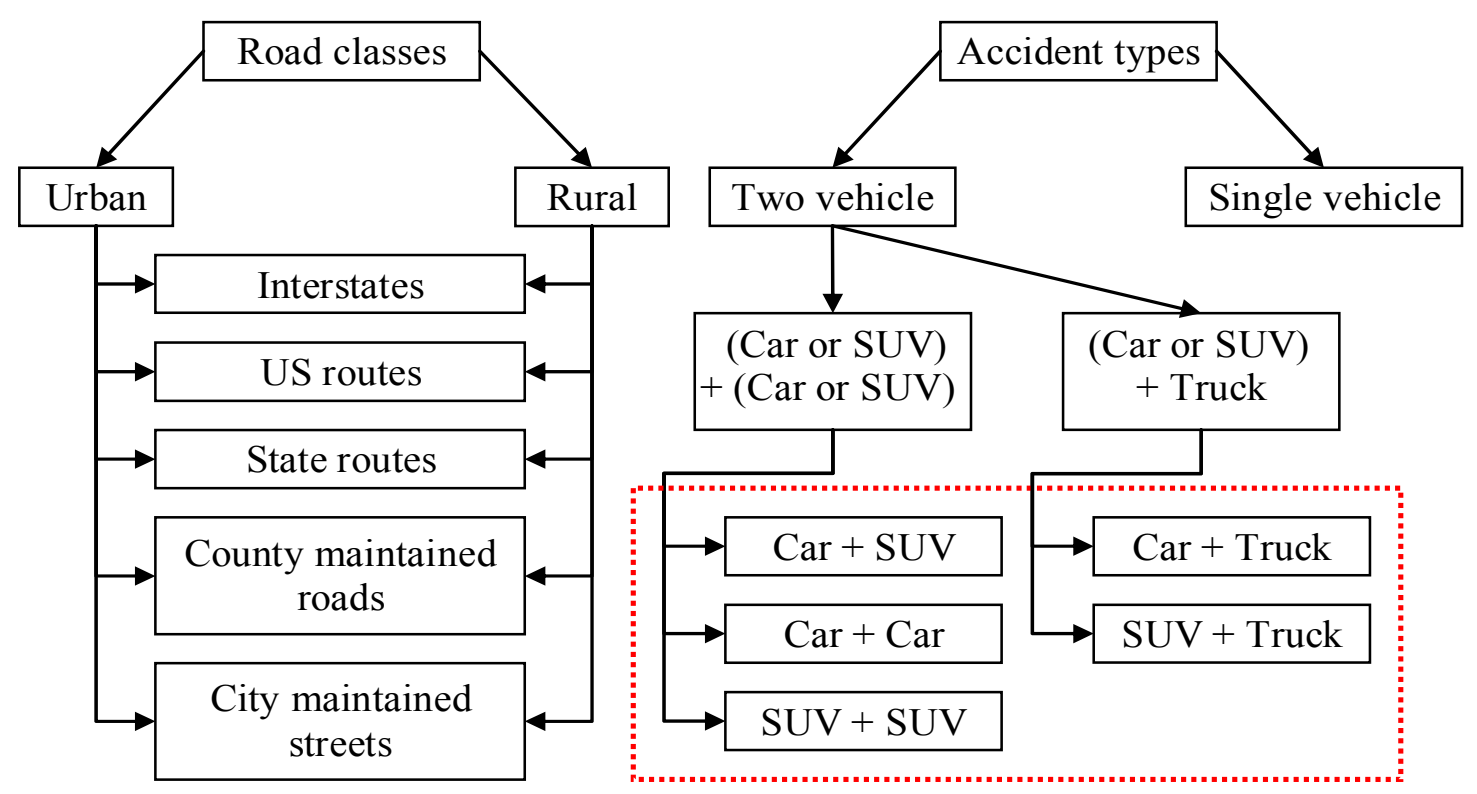

"SUV" means sport utility vehicles, pickups and vans. "Truck" means any possible kind of a truck or a tractor. SUVs and cars are considered together unless their additional division, as shown inside the dotted box, is required by the likelihood ratio test.

Figure 4.1 Data division by road class and by accident type ${ }^{7}$

We check statistical significance of the explanatory variables in all logit models by using $5 \%$ significance level for the two-tailed t-test of a large data sample. In other words, coefficients with t-ratios between -1.96 and +1.96 are considered

${ }^{7}$ We consider US routes and State routes separately even though they have similar design and other properties. The reason is that our final logit models for unsafe-speed-related accident causation on US and State routes turn out to be statistically different from each other. We use the likelihood ratio test to check this difference (but we do not report the test results in this herein). 
to be statistically insignificant. Note that the explanatory variables can be mutually dependent (e.g. a quantitative variable and its derivative indicator variable are strongly mutually dependent).

Statistical models are (usually) estimated by maximizing the model's loglikelihood function. However, one can not rely on the log-likelihood maximization alone in order to choose the optimal number of explanatory variables to be included into a statistical model. The reason is that the log-likelihood (LL) function is always maximized when all available explanatory variables are included into the model. This is because a removal of any explanatory variable is equivalent to restricting its value to zero, which always either decreases the maximum of LL or leaves it the same. As a result, in the present study we use the Akaike Information Criterion (AIC), minimization of which ensures an optimal choice of explanatory variables in a model (Tsay, 2002, page 37; Washington et al., 2003, page 212; Wikipedia). The main idea behind the AIC is to examine the complexity of a model together with goodness of its fit to the data sample, and to find a balance between the two. A model with too few explanatory variables will provide a poor fit to the data sample. A model with too many variables will provide a very good fit, but will lack necessary robustness and will perform poorly in out-of-the-sample data. The preferred model with the optimal number of explanatory variables is the model with the lowest AIC value, which is given by equation

$$
A I C=-2 L L+2 K,
$$

where LL is the log-likelihood value of a model, and $\mathrm{K}$ is the number of estimable coefficients in the model (one coefficient for each explanatory variable, including the intercepts).

In our research we estimate all logit models by using one of the two procedures $A$ and $B$ shown in Figure 4.2. Procedure $A$ is as follows: 
Procedure A

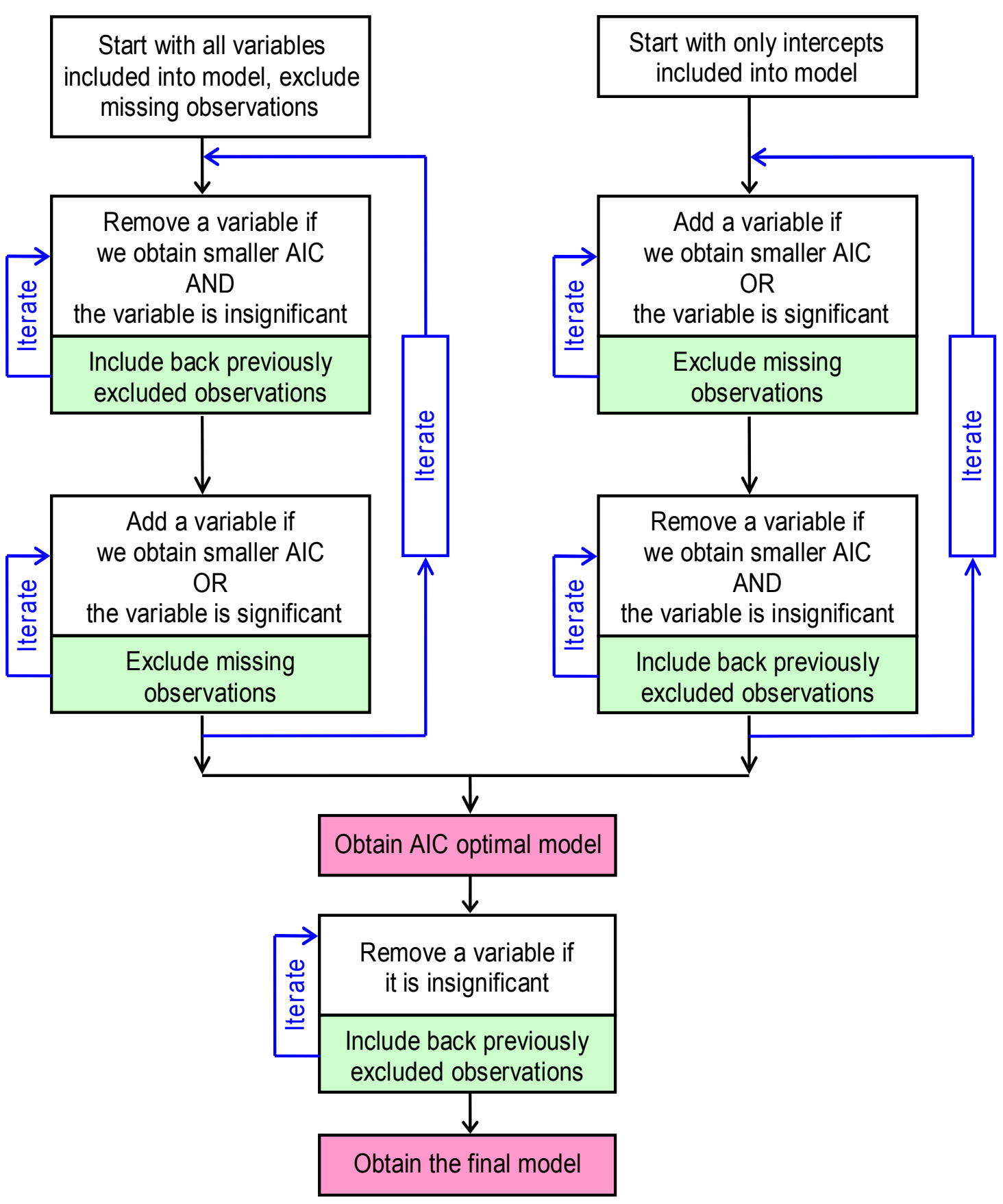

Figure 4.2 Model estimation procedures 
A. We start with all explanatory variables initially included into a logit model. Note that, when estimating a model, we have to exclude observations that are missing for any of the included variables. Next, we obtain the final model by using three steps of model estimation. The first step is

1. We remove the least statistically significant explanatory variables (as judged by their t-ratios) one by one if both of the following two conditions are satisfied: the removal of a variable decreases the AIC value and the removed variable is statistically insignificant (under the $5 \%$ confidence level) ${ }^{8}$. Note that while using the Akaike information criterion, we always keep the number of data sample observations constant in order to calculate the changes of the AIC value correctly. Each time when we have removed several (usually four) least significant explanatory variables from a model, we include some of the previously excluded observations back into the data sample because now the model includes fewer variables with missing observations. We keep removing insignificant explanatory variables one by one, periodically including previously excluded observations back into the data sample, until we can not remove any additional variable under the two conditions listed above.

After we removed all variables that we could, we need to check if any of the removed variables can be added back into the model. This is because variables are mutually dependent and "interact" in the model. Therefore, we proceed to the second step of model estimation:

8 If the asymptotic normality of maximum likelihood estimates holds, then the AIC value does not change with removal (addition) of a variable whose coefficient has $15.73 \% \mathrm{p}$-value for the two-tailed test (15.73\% $p$-value corresponds to $\pm \sqrt{2}$ t-ratio for a normal variate). In this case the $5 \%$ confidence level test of the variable is redundant, and the AIC test alone can be used for removal and addition of variables in model estimation steps 1 and 2. Nevertheless, we use both tests to make our estimation procedures more robust in case the normality of maximum likelihood estimates does not hold. 
2. We add explanatory variables one by one if at least one of the following two conditions is satisfied: either the addition of a variable decreases the AIC value or the added variable is significant $^{9}$. As usual, the AIC values are compared under the condition that the number of observations is kept constant. As the number of the explanatory variables included into the model grows, the data sample size shrinks because of a larger number of missing observations associated with the included variables. We add explanatory variables one by one until no any additional variable can be added to the model.

Next we return back to the first estimation step given above and remove variables that can be removed. We iterate between steps 1 and 2 until we can neither remove nor add any more variables. At this point we arrive at the model that we call the "AIC optimal model" (refer to Figure 4.2). Next, we proceed to the third and final step of model estimation:

3. To make our final results more robust, we drop from the AIC optimal model all remaining statistically insignificant variables (judged by the $5 \%$ significance level for the two-tailed t-test). As a result, we obtain the final model, which is our best model (according to the estimation procedures chosen by us).

Now we describe procedure B:

B. In this procedure we start with only intercepts (constant terms) initially included into a logit model (refer to Figure 4.2). Next, we proceed in a way very similar to that used in procedure A. We run step 2 of model estimation and add explanatory variables into the model. Then, we iterate between steps 1 and 2 until we can neither remove nor add any more variables, at which point we arrive at the AIC optimal model. Finally, we run step 3 of model estimation and obtain the best final model.

\footnotetext{
${ }^{9}$ We first search for and add AIC decreasing variables, and afterwards we add significant variables if there are any.
} 
By default we always use procedure A for model estimation, and only if we can not use it (usually when the available data sample is too small for the initial model estimation with all explanatory variables included), then we resort to procedure $B$.

\subsection{Results: accident causation models}

For each of the road-class-accident-type combinations listed in Table B.1 and Table B.2 in Appendix B, we find and estimate the best binary logit model by using either procedure $A$ or procedure $B$ described above. The binary logit models are given in Equation (2.3), where outcome "1" corresponds to the case when the primary cause of an accident is either "unsafe speed" or "speed too fast for weather conditions", and outcome " 2 " corresponds to any other primary cause of the accident. The results of the estimation of the best models are given in Table B. 3 and Table B.4 for 2004 and 2006 accidents respectively (see Appendix B).

In Table B.5 and Table B.6 in Appendix B we give the results of testing whether, in 2004 and 2006 two-vehicle accidents, cars and SUVs can be considered together or must be considered separately. This testing is done for the best models by using the likelihood ratio test given in Equation (2.5). According to the results shown in Table B.5 and Table B.6, we find that in our unsafe-speedrelated accident causation study cars and SUVs can be considered together in all 2004 two-vehicle accidents on all road classes, but they must be considered separately in the case of several road-class-accident-type combinations for 2006 two-vehicle accidents.

Let us now examine the model estimation results, which are given in Table B.3 and Table B.4 for 2004 and 2006 accidents respectively. We will consider the effects of the posted speed limit and other explanatory variables on the 
probability of an unsafe and/or excessive speed being the primary cause of an accident. Since our primary interest is the effect of the speed limit, we focus on it first.

\subsubsection{Effect of Speed Limit}

We assume that the speed limit posted at the location of an accident is known only if it is indicated as known and the same for all vehicles involved into the accident. The speed limit is variable $X_{29}$ in Appendix A. Its coefficients and averaged elasticities in the best final binary logit models for 2004 and 2006 unsafe-speed-related accident causation are given in Table 4.1 and Table 4.2 below. In order to understand the results reported in these tables, please refer to Equations (2.3) and (2.8). These equations give the binary logit model and the corresponding elasticities that we calculate. The outcomes "1" and "2" in the binary models correspond to the "unsafe-speed-related cause" and "any other cause" of an accident. In Table 4.1 and Table 4.2 we report all statistically significant coefficients of the speed limit variable (these coefficients are copied from Table B. 3 on page 83 and Table B.4 on page 94) and the corresponding elasticities. In addition, in these tables we report all statistically insignificant coefficients of the speed limit variable (without elasticities). These insignificant coefficients are shown in the square brackets and are obtained by test-adding the speed limit variable into the AIC optimal logit models (note that this is done only as a test; in Table 4.1, Table 4.2, Table B.3 and Table B.4 all significant coefficients and the corresponding elasticities are reported for the final models, which themselves do not contain any insignificant variables).

We find the following results for the effects of speed limit on accident causation: 
Table 4.12004 accident causation models: results for speed limit ${ }^{10}$

\begin{tabular}{|c|c|c|c|c|c|c|}
\hline \multirow[t]{2}{*}{ \# } & \multirow{2}{*}{\multicolumn{3}{|c|}{ Model name }} & \multirow{2}{*}{$\begin{array}{c}\text { Speed limit } \\
\text { coefficient (t-ratio) }\end{array}$} & \multicolumn{2}{|c|}{$\begin{array}{c}\text { Averaged elasticities of } \\
\text { speed limit (SL) }\end{array}$} \\
\hline & & & & & $\bar{E}_{1 ; S L}^{(1)}$ & $\bar{E}_{1 ; S L}^{(2)}$ \\
\hline 1 & \multirow{3}{*}{\multicolumn{2}{|c|}{$\mid \begin{array}{l}\overline{0} \\
\frac{\pi}{2} \\
0 \\
0\end{array}$}} & (car/SUV)+(car/SUV) & {$[.00943(1.67)]$} & & \\
\hline 2 & & & (car/SUV)+(truck) & {$[-.00555(-.223)]$} & & \\
\hline 3 & & & one vehicle & $.00859(2.83)$ & .337 & -.061 \\
\hline 4 & \multirow{3}{*}{ نे } & \multirow{3}{*}{\begin{tabular}{|l|} 
\\
$\frac{c}{\widetilde{D}}$ \\
$\frac{0}{J}$ \\
\end{tabular}} & (car/SUV)+(car/SUV) & $.0368(2.24)$ & 1.23 & -.094 \\
\hline 5 & & & (car/SUV)+(truck) & {$[.177(1.43)]$} & & \\
\hline 6 & & & one vehicle & {$[.00986(.827)]$} & & \\
\hline 7 & \multirow{3}{*}{\multicolumn{2}{|c|}{ 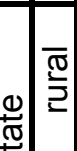 }} & (car/SUV)+(car/SUV) & {$[.0172(1.27)]$} & & \\
\hline 8 & & & (car/SUV)+(truck) & {$[-.0127(-.317)]$} & & \\
\hline 9 & & & one vehicle & {$[-.0294(-1.95)]$} & & \\
\hline 10 & \multirow{3}{*}{ 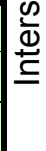 } & \multirow{3}{*}{\begin{tabular}{l|}
$\frac{c}{\widetilde{D}}$ \\
$\frac{0}{3}$ \\
$\frac{2}{J}$
\end{tabular}} & (car/SUV)+(car/SUV) & {$[.0182(1.19)]$} & & \\
\hline 11 & & & (car/SUV)+(truck) & {$[.0539(1.42)]$} & & \\
\hline 12 & & & one vehicle & {$[-.00985(-.873)]$} & & \\
\hline 13 & \multirow{3}{*}{\multicolumn{2}{|c|}{ 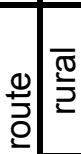 }} & (car/SUV)+(car/SUV) & {$[-.00230(-.175)]$} & & \\
\hline 14 & & & (car/SUV)+(truck) & {$[.00806(.254)]$} & & \\
\hline 15 & & & one vehicle & {$[-.0108(-1.30)]$} & & \\
\hline 16 & \multirow{3}{*}{ 岏 } & \multirow{3}{*}{\begin{tabular}{l|}
\multicolumn{1}{c|}{} \\
त్ \\
잏 \\
\end{tabular}} & (car/SUV)+(car/SUV) & $.0212(2.51)$ & .769 & -.034 \\
\hline 17 & & & (car/SUV)+(truck) & {$[.0337(1.05)]$} & & \\
\hline 18 & & & one vehicle & {$[.000799(.066)]$} & & \\
\hline 19 & \multirow{3}{*}{\multicolumn{2}{|c|}{ 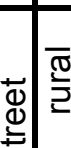 }} & (car/SUV)+(car/SUV) & $.0225(2.50)$ & .773 & -.050 \\
\hline 20 & & & (car/SUV)+(truck) & {$[.102(1.25)]$} & & \\
\hline 21 & & & one vehicle & {$[-.00158(-.248)]$} & & \\
\hline 22 & \multirow{3}{*}{$\underset{0}{\infty}$} & \multirow{3}{*}{$\begin{array}{l}\frac{c}{\pi} \\
\frac{0}{J} \\
\frac{3}{5}\end{array}$} & (car/SUV)+(car/SUV) & {$[-.00504(-1.14)]$} & & \\
\hline 23 & & & (car/SUV)+(truck) & {$[.0113(.543)]$} & & \\
\hline 24 & & & one vehicle & $-.0117(-3.82)$ & -.323 & .053 \\
\hline 25 & \multirow{3}{*}{\multicolumn{2}{|c|}{\begin{tabular}{l|l}
$\overline{\frac{\pi}{\sigma}}$ \\
$\frac{\Phi}{3}$
\end{tabular}}} & (car/SUV)+(car/SUV) & {$[.00721(.509)]$} & & \\
\hline 26 & & & (car/SUV)+(truck) & {$[-.0356(-1.23)]$} & & \\
\hline 27 & & & one vehicle & $-.0422(-3.17)$ & -2.05 & .204 \\
\hline 28 & \multirow{3}{*}{ 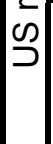 } & \multirow{3}{*}{$\begin{array}{l}\frac{c}{\sqrt{0}} \\
\frac{0}{\zeta}\end{array}$} & (car/SUV)+(car/SUV) & $.0181(2.33)$ & .679 & -.040 \\
\hline 29 & & & (car/SUV)+(truck) & {$[.0517(1.50)]$} & & \\
\hline 30 & & & one vehicle & {$[.00636(.394)]$} & & \\
\hline
\end{tabular}

10 Refer to Equations (2.3) and (2.8), where outcomes "1" and "2" correspond to the "unsafespeed-related" and "any other" accident causes. We report statistically significant coefficients of the speed limit variable and the corresponding elasticities. In addition, in the square brackets we report statistically insignificant coefficients (obtained by test-adding the speed limit variable into the AIC optimal models). All coefficients are the components of vector $\boldsymbol{\beta}_{1}$ that are multiplied by the speed limit variable in Equation (2.3). 
Table 4.2 2006 accident causation models: results for speed limit

\begin{tabular}{|c|c|c|c|c|c|c|}
\hline \multirow[t]{2}{*}{$\#$} & & & \multirow{2}{*}{ Model name } & \multirow{2}{*}{$\begin{array}{c}\text { Speed limit } \\
\text { coefficient (t-ratio) }\end{array}$} & \multicolumn{2}{|c|}{$\begin{array}{c}\text { Averaged elasticities of } \\
\text { speed limit (SL) }\end{array}$} \\
\hline & & & & & $\overline{E_{1 ; S L}^{(1)}}$ & $\bar{E}_{1 ; S L}^{(2)}$ \\
\hline 1 & \multirow{4}{*}{\multicolumn{2}{|c|}{ 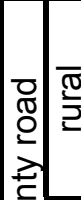 }} & (car/SUV)+(car/SUV) & {$[-.0222(-1.57)]$} & & \\
\hline $2 a$ & & & (car)+(truck) & {$[-.115(-1.36)]$} & & \\
\hline $2 b$ & & & (SUV)+(truck) & {$[.246(.917)]$} & & \\
\hline 3 & & & one vehicle & {$[.000990(.155)]$} & & \\
\hline 4 & \multirow{3}{*}{ రે } & \multirow{3}{*}{$\begin{array}{l}\text { 孚 } \\
\frac{0}{5} \\
\frac{\partial}{J}\end{array}$} & (car/SUV)+(car/SUV) & {$[-.0101(-.309)]$} & & \\
\hline 5 & & & (car/SUV)+(truck) & {$[0.00792(.113)]$} & & \\
\hline 6 & & & one vehicle & {$[-.00975(-.755)]$} & & \\
\hline $7 a$ & & \multirow{5}{*}{$\frac{\overline{0}}{\underline{\underline{T}}}$} & $($ car $)+($ car $)$ & {$[-.00840(-.346)]$} & & \\
\hline $7 b$ & & & (car)+(SUV) & {$[.0110(.569)]$} & & \\
\hline $7 \mathrm{c}$ & & & $(S U V)+(S U V)$ & {$[.0296(.958)]$} & & \\
\hline 8 & $\stackrel{ \pm}{\pi}$ & & (car/SUV)+(truck) & {$[-.0176(-.816)]$} & & \\
\hline 9 & $\frac{\pi}{0}$ & & one vehicle & $-.0439(-5.72)$ & -2.51 & .370 \\
\hline 10 & 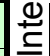 & \multirow{4}{*}{ 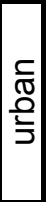 } & (car/SUV)+(car/SUV) & {$[.0232(1.85)]$} & & \\
\hline $11 \mathrm{a}$ & & & (car)+(truck) & {$[-.0479(-1.49)]$} & & \\
\hline $11 \mathrm{~b}$ & & & (SUV)+(truck) & {$[.113(1.27)]$} & & \\
\hline 12 & & & one vehicle & {$[-.0158(-.811)]$} & & \\
\hline 13 & & \multirow{3}{*}{ 苞 } & (car/SUV)+(car/SUV) & {$[.000526(.0230)]$} & & \\
\hline 14 & $\stackrel{\oplus}{\stackrel{\Phi}{7}}$ & & (car/SUV)+(truck) & {$[.00874(.284)]$} & & \\
\hline 15 & $\underline{\underline{0}}$ & & one vehicle & $-.0373(-5.34)$ & -1.87 & .109 \\
\hline 16 & $\stackrel{\frac{0}{\pi}}{\pi}$ & \multirow{3}{*}{$\begin{array}{l}\frac{5}{\pi} \\
\frac{0}{\grave{J}}\end{array}$} & (car/SUV)+(car/SUV) & $.0277(3.47)$ & .999 & -.0390 \\
\hline 17 & ஸे & & (car/SUV)+(truck) & {$[.0250(.907)]$} & & \\
\hline 18 & & & one vehicle & {$[-.0102(-1.000)]$} & & \\
\hline 19 & & \multirow{4}{*}{ 离 } & (car/SUV)+(car/SUV) & {$[-.00521(-.241)]$} & & \\
\hline $20 a$ & & & (car)+(truck) & {$[.0239(.381)]$} & & \\
\hline $20 \mathrm{~b}$ & + & & (SUV)+(truck) & {$[-.142(-.634)]$} & & \\
\hline 21 & $\stackrel{\Phi}{=}$ & & one vehicle & {$[.00475(.612)]$} & & \\
\hline 22 & $\prod_{\geq}^{\infty}$ & \multirow{4}{*}{\begin{tabular}{|l|}
$\frac{c}{\mathbb{0}}$ \\
$\frac{0}{5}$ \\
$\frac{5}{5}$
\end{tabular}} & (car/SUV)+(car/SUV) & {$[.00811(1.010)]$} & & \\
\hline $23 a$ & $\overline{0}$ & & (car)+(truck) & {$[.0325(.889)]$} & & \\
\hline $23 b$ & & & $(\mathrm{SUV})+($ truck $)$ & {$[.118(1.910)]$} & & \\
\hline 24 & & & one vehicle & {$[.00226(.301)]$} & & \\
\hline 25 & & \multirow{3}{*}{$\frac{\bar{\pi}}{\stackrel{2}{2}}$} & (car/SUV)+(car/SUV) & {$[.00969(.321)]$} & & \\
\hline 26 & & & (car/SUV)+(truck) & {$[.00144(.042)]$} & & \\
\hline 27 & $\stackrel{0}{5}$ & & one vehicle & {$[-.00537(-.464)]$} & & \\
\hline 28 & ō & \multirow{4}{*}{ 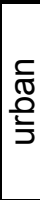 } & (car/SUV)+(car/SUV) & {$[.0122(.646)]$} & & \\
\hline $29 a$ & D & & (car)+(truck) & {$[-.0127(-.294)]$} & & \\
\hline $29 b$ & & & (car)+(truck) & {$[.362(1.73)]$} & & \\
\hline 30 & & & one vehicle & {$[-.0101(-.814)]$} & & \\
\hline
\end{tabular}


- Speed limit does not have any statistically significant effect on unsafespeed-related causation of accidents of any types ${ }^{11}$ on interstate highways (urban and rural), except for the case of 2006 one-vehicle accidents on rural interstates. In this single case the probability of unsafe speed being the primary cause of an accident actually decreases with an increase in the posted speed limit.

- Speed limit does not also have a statistically significant effect on unsafespeed-related accident causation for the majority of other accident types on the majority of road classes other than the interstate highways.

- There are only ten combinations of different accident types and road classes for which speed limit turns out to have a statistically significant effect on unsafe-speed-related causation of 2004 and 2006 accidents. For convenience, in

- Figure 4.3 and Figure 4.4 we present in graphical form the t-ratios of the speed limit coefficients for these ten combinations. We see that there are mixed effects of the speed limit on unsafe-speed-related accident causation. On one hand, the probability of unsafe speed being the primary cause of an accident rises with an increase in the posted speed limit for 2004 single-vehicle accidents on rural county maintained roads, for $2004 \mathrm{car} / \mathrm{SUV}$-car/SUV accidents on rural city maintained streets, urban US routes and urban county maintained roads, as well as for 2004 \& $2006 \mathrm{car} / \mathrm{SUV}$-car/SUV accidents on urban state routes. On the other hand, the probability decreases with an increase in the speed limit for 2004 single-vehicle accidents on rural US routes and urban city maintained streets, and for 2006 single-vehicle accidents on rural state routes and rural interstates.

- The speed limit variable is elastic for only one of the six road-classaccident-type combinations that display a statistically significant increase

\footnotetext{
11 Note that we consider only single-vehicle accidents and all two-vehicle accidents except those that involve two trucks.
} 
of the unsafe speed accident causation probability with an increase of the posted speed limit (this single combination is 2004 car/SUV-car/SUV accidents on urban county maintained roads).

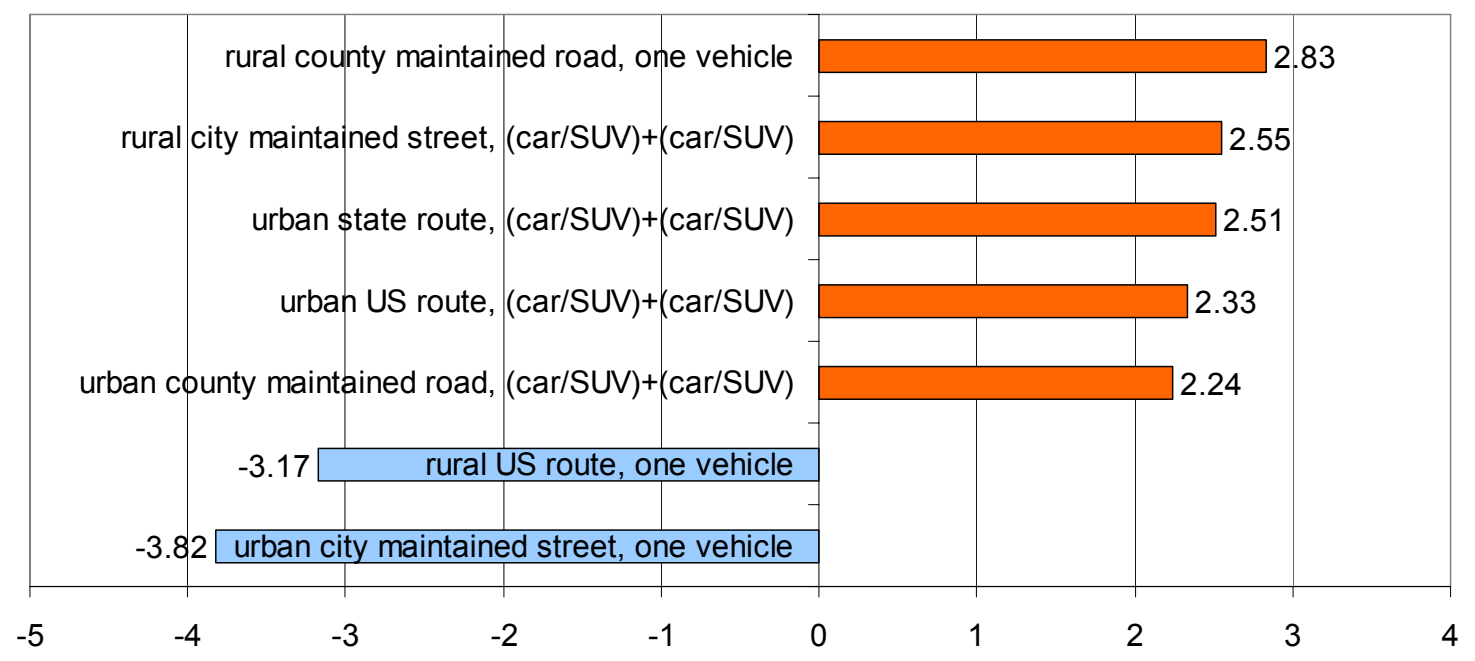

Figure 4.3 T-ratios of statistically significant speed limit coefficients in 2004 accident causation models

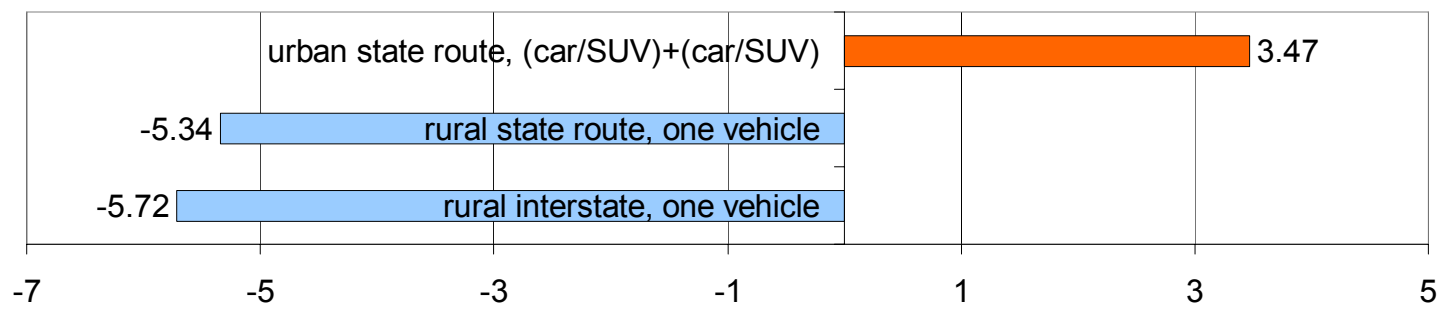

Figure 4.4 T-ratios of statistically significant speed limit coefficients in 2006 accident causation models

We postpone a discussion of the above findings until the last chapter, which discusses our results for both accident causation and accident severity. 


\subsubsection{Effect of Other Explanatory Variables}

Now we use model estimation results given in Table B.3 and Table B.4 in Appendix $B$ to study the influence of explanatory variables other than the posted speed limit on unsafe-speed-related causation of 2004 and 2006 accidents. We limit our consideration to several of the most important variables, which are statistically significant not just in a few but in many models for different road classes and accident types ${ }^{12}$.

- Variable "wint" (see pages 84 and 95): We find that the probability of unsafe-speed-related cause of an accident increases during winter season. This seasonal effect is very strong. It is reasonable because driving conditions in Indiana worsen during winters, while some drivers apparently fail to adjust their driving speeds accordingly.

- Variables "precip", "snow" and "dry" (see pages 86 and 97): We find that the probability of unsafe-speed-related cause of an accident increases when precipitation and/or snow is observed at the accident location, and it decreases when the road surface is dry. This is a very strong effect on all road classes, and it can clearly be explained by drivers not being adjusting their speed appropriately when the weather conditions are less safe for driving.

- Variable "nojun" (see pages 87 and 98): The absence or presence of a road junction at the accident location has a mixed effect on the probability of unsafe-speed-related cause of an accident. The probability of an unsafe-speed-related accident with only one vehicle involved decreases when no junction is present on the road, but the probability increases when two vehicles are involved. This can be explained by two concurrent effects. On one hand, the road is safer in the absence of

\footnotetext{
12 One of the reasons for this limitation is that the number of available accident observations in our data sample is relatively small for several road-class-accident-type combinations, refer to Table B.1 and Table B.2.
} 
junctions. On the other hand, drivers might have a habit of slowing down a bit when they approach a junction and see another vehicle(s) on or near the junction ${ }^{13}$.

- Variables "curve" (see pages 88 and 99): We find an expected result that the probability of unsafe-speed-related cause of an accident decreases when the road is straight and increases when the road is at curve. Clearly curved roads are less safe because of centrifugal forces acting onto moving vehicles in the rotating coordinate frame of reference.

- Variable "heavy" (see pages 89 and 101): We find that heavy trucks and tractors are less likely to cause unsafe-speed-related accidents than other vehicles are. The reason can be that drivers of trucks and tractors are more professional and are less likely to speed.

- Variables "stopsig" (see pages 92 and 103): We find that the presence of a stop sign generally reduces the probability of unsafespeed-related accidents, which can be due to effectiveness of stop signs in controlling traffic flows on streets and minor roads.

- Variable " $X_{34}$ " (see pages 93 and 104): We find that the probability of unsafe-speed-related cause of an accident decreases with the age of the driver at fault. This is a very strong effect on all road classes. Apparently older drivers are more experienced, more careful and much less likely to exceed safe driving speed.

\footnotetext{
${ }^{13}$ Note that police officer's misidentification errors can be stronger for a single-vehicle accident because in this case the officer has to rely on testimonies of occupants of the single vehicle involved into the accident. See footnote 4 on page 17 for discussion of misidentification errors.
} 


\section{CHAPTER 5. ACCIDENT SEVERITY STUDY}

In this chapter our goal is to reveal and study the impact of the posted speed limit on the severity level of an accident. Similar to the previous chapter, we first describe the procedures of accident severity statistical modeling that we use, and second, we present and discuss the results that we obtain for 2004 and 2006 accidents. In our analysis, we will compare the cross-sectional effect of speed limits in each accident year. We do not restrict our analysis to the change in accident severity only roadway segments that had increased speed limits. If he had done so, the natural variation in accidents over time during the two-year period (driving behavior, vehicle safety features, etc) would have masked the effect of speed limits. Instead, by using a cross-sectional analysis and controlling for all characteristics of accidents (driver characteristics, weather conditions, roadway geometrics, urban/rural location, etc.) we are able to isolate the effects of speed-limit variations in a given accident year.

\subsection{Modeling Procedures: accident severity}

For each accident, the severity level is determined by the injury level sustained by the most injured individual (if any) involved into the accident. By using the available individual accident data, we are able to distinguish between three levels of accident severity. Listed in increasing order, these are

1. no-injury or property damage only (PDO),

2. injury,

3. fatality, 
refer to Figure 3.4 and Figure 3.10. As a result, for the statistical modeling of accident severity we use a multinomial logit model with three possible outcomes that correspond to these three levels of accident severity. This multinomial logit model is given by Equation (2.4), where the outcomes "1", "2" and " 3 " correspond to "fatality", "injury" and "PDO" levels of accident severity respectively.

We estimate multinomial logit models for accident severity in a way similar to the estimation of binary logit models for accident causation considered in the previous chapter. Namely, we again consider different road classes and accident types separately, as shown in Figure 4.1. The list of all combinations of different road classes and accident types that we consider in our accident severity study can be found in Table C.1 and Table C.2 for 2004 and 2006 accidents respectively (see Appendix $\mathrm{C}$ ). We again use $5 \%$ significance level for the two-tailed t-test of a large data sample in order to make inference on statistical significance of all indicator and quantitative explanatory variables in the accident severity logit models. We also use the same AIC-based procedures (A and B) for all severity model estimations, as described in CHAPTER 4 on pages 32 and 33 and in Figure 4.2.

Thus, to study the impact of speed limit on the resulting accident severity, for each road-class-accident-type combination we proceed as follows:

1. First, using the data on accidents that constitute the considered roadclass-accident-type combination and the procedures described above, we find the best multinomial logit model with three possible accident severity outcomes (fatality, injury, PDO). From this model we can immediately see whether there is any statistically significant effect of the posted speed limit on the resulting accident severity level. 
2. Second, we divide the accident data into separate speed limit data bins, according to the posted speed limit at the place of an accident ${ }^{14}$. The speed limit bins chosen by us for 2004 and 2006 accidents are given in Table C.3 and Table C.4 in Appendix C.

3. Third, we take the best logit model obtained in the first $\operatorname{step}^{15}$ and reestimate it separately for each of the speed-limit data bins chosen in the second step. Then we test to see if there are statistically significant differences among the models estimated for the different speed-limit bins. This is done by using the likelihood ratio test, which is given by Equation (2.5) and explained in the end of CHAPTER 2. We use $5 \%$ confidence level for the likelihood ratio test statistic in Equation (2.5) to make inference on whether the collection of models estimated separately for the speed-limit data bins is statistically the same as the model estimated for the whole data sample which includes all speed limits together. In other words, if the left-hand-side of Equation (2.5) is between zero and the $95^{\text {th }}$ percentile of the chi-squared distribution given on the right-hand-side, then we conclude that the posted speed limit makes no statistically significant difference for the structure of accident severity models in the case of the considered road-class-accident-type combination. We conclude that there is a difference otherwise.

\subsection{Results: accident severity models}

For each of the road-class-accident-type combinations listed in Table C.1 and Table C.2 in Appendix C, we find and estimate the best multinomial logit model, as given in Equation (2.3) with the outcomes "1", "2" and "3" corresponding to

\footnotetext{
${ }^{14}$ We disregard accidents with more than one posted speed limit at the accident location.

15 In the third step we first remove the speed limit variable (if any) from the best final model because the speed limit is usually constant inside the speed limit data bins. In some cases of the 2004 accident severity models we have to remove additional explanatory variables that are constant inside some of the speed limit bins. For specific cases of the removal see Table 5.3.
} 
"fatality", "injury" and "PDO" accident severity levels respectively. Table C.5 and Table C.6 in Appendix C give the estimation results for the best models in the cases of 2004 and 2006 accidents.

In Table C.7 and Table C.8 we show the results of testing whether in twovehicle accidents cars and SUVs can be considered together or must be considered separately in our accident severity study. This testing is done by using the likelihood ratio test in exactly the same way as done in the accident causation study. If the likelihood ratio test indicates that cars and SUVs should be considered separately, then we apply the additional division shown inside the dotted box in Figure 3.2 and find the best model separately for each of the subcategories obtained by this additional division. For example, from the test results given in Table C.7 we see that the 2004 "car/SUV-truck accidents on rural interstates" category has to be divided into sub-categories 8a (car-truck accidents) and 8b (SUV-truck accidents).

\subsubsection{Effect of Speed Limit}

To judge whether speed limit makes any statistically significant difference for the resulting accident severity outcomes, we first study the severity model estimation results for the speed limit variable and its elasticities reported in Table 5.1 and Table 5.2 for 2004 and 2006 accidents respectively. In order to understand the results presented in these tables, refer to Equations (2.4) and (2.9), where outcomes "1", "2" and "3" correspond to "fatality", "injury" and "PDO" accident severity outcomes respectively. In Table 5.1 and Table 5.2 the elasticities are reported only for statistically significant coefficients of the speed limit variable (which are copied from Table C.5 on page 112 and Table C.6 on page 142). In Table 5.1 and Table 5.2 we also report all statistically insignificant coefficients of the speed limit variable, which are shown in the square brackets and are obtained by test-adding the speed limit variable into the AIC optimal 
models (note that this is done only as a test; in Table5.1, Table 5.2, Table C.5 and Table C.6 all significant coefficients and the corresponding elasticities are reported for the final models, which themselves do not contain any insignificant variables).

We find that

- Speed limit did not have any statistically significant effect on severity of 2004 and 2006 accidents of any type ${ }^{16}$ on interstate highways (both urban and rural). This indicates that the speed-limit increases in $2005 \mathrm{did}$ not affect the relative severity of accidents on interstates with different speed limits.

- Higher speed limit values did generally lead to higher probabilities of more severe accidents (fatality and injury) on road classes other than interstate highways. This effect is especially strong for 2004 and 2006 accidents on rural country roads, urban city maintained streets and urban U.S. routes.

- The speed limit variable seems to be inelastic for some of the road-classaccident-type combinations that display a statistically significant relationship between speed limit and accident severity. For others, especially in the case of fatal accident outcomes, elasticities of the speed limit can be quite high.

\footnotetext{
16 Remember that we consider only single- and two-vehicle accidents (except for two-truck accidents).
} 
Table 5.12004 accident severity models: results for speed limit ${ }^{17}$

\begin{tabular}{|c|c|c|c|c|c|c|c|c|c|}
\hline \multirow{2}{*}{ \# } & \multirow{2}{*}{\multicolumn{3}{|c|}{ Model name* }} & \multicolumn{2}{|c|}{$\begin{array}{c}\text { Speed limit } \\
\text { coefficient (t-ratio) }\end{array}$} & \multicolumn{4}{|c|}{$\begin{array}{l}\text { Averaged elasticities of } \\
\text { speed limit (SL) }\end{array}$} \\
\hline & & & & & & & & & $\bar{E}_{2: S L}^{(1)}=$ \\
\hline 1 & & & $(\mathrm{C} / \mathrm{S})+(\mathrm{C} / \mathrm{S})$ & $.108(3.61)$ & $.0255(5.15)$ & 4.49 & -.042 & .776 & -.297 \\
\hline 2 & 半 & & $(\mathrm{C} / \mathrm{S})+(\mathrm{T})$ & {$[.337(1.34)]$} & $.0414(3.42)$ & & & 1.35 & -.405 \\
\hline 3 & $\stackrel{2}{2}$ & & one vehicle & $.0382(3.47)$ & {$[-.00315(-1.03)$} & 1.75 & -.022 & & \\
\hline 4 & डी & & $(\mathrm{C} / \mathrm{S})+(\mathrm{C} / \mathrm{S})$ & $.0323(3.75)$ & $.0323(3.75)$ & 1.19 & -.001 & .927 & -.259 \\
\hline 5 & & & $(\mathrm{C} / \mathrm{S})+(\mathrm{T})$ & $-.00511(.000)]$ & {$[.0536(1.08)]$} & & & & \\
\hline 6 & & & one vehicle & {$[-.0974(-.853)]$} & {$[.00469(.354)]$} & & & & \\
\hline 7 & & & $(\mathrm{C} / \mathrm{S})+(\mathrm{C} / \mathrm{S})$ & $.000351(.000)]$ & {$[.0116(.646)]$} & & & & \\
\hline $8 a$ & & ๘) & $(\mathrm{C})+(\mathrm{T})$ & {$[.00133(.000)]$} & {$[.00870(.358)]$} & & & & \\
\hline $8 b$ & 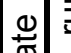 & & $(\mathrm{S})+(\mathrm{T})$ & {$[.0272(.000)]$} & {$[.0374(1.33)]$} & & & & \\
\hline 9 & 童 & & one vehicle & {$[-.0186(-1.49)]$} & {$[-.0186(-1.49)]$} & & & & \\
\hline$\overline{10}$ & & & $(\mathrm{C} / \mathrm{S})+(\mathrm{C} / \mathrm{S})$ & {$[.0171(1.41)]$} & {$[.0171(1.41)]$} & & & & \\
\hline 11 & & & $(\mathrm{C} / \mathrm{S})+(\mathrm{T})$ & {$[.0798(.518)]$} & {$[.000717(.026)]$} & & & & \\
\hline 12 & & 5 & one vehicle & {$[.0366(.938)]$} & {$[-.00262(-.240)]$} & & & & \\
\hline 13 & & & $(\mathrm{C} / \mathrm{S})+(\mathrm{C} / \mathrm{S})$ & {$[.0628(1.43)]$} & $.0306(3.90)$ & & & 1.03 & -.505 \\
\hline 14 & & & $(\mathrm{C} / \mathrm{S})+(\mathrm{T})$ & {$[.168(1.88)]$} & {$[.0239(1.36)]$} & & & & \\
\hline 15 & ه & & one vehicle & {$[.0313(1.09)]$} & {$[-.00313(-.422)$} & & & & \\
\hline $16 a$ & 항 & & $(\mathrm{C})+(\mathrm{C})$ & $.0340(5.14)$ & $.0340(5.14)$ & 1.27 & -.001 & .949 & -.319 \\
\hline $16 \mathrm{~b}$ & & & $(\mathrm{C})+(\mathrm{S})$ & $.0225(4.36)$ & $.0225(4.36)$ & .853 & -.001 & .652 & -.201 \\
\hline $16 \mathrm{c}$ & & & $(\mathrm{S})+(\mathrm{S})$ & .0315 (3.39) & .0315 (3.39) & 1.21 & -.005 & .951 & -.265 \\
\hline 17 & & & $(\mathrm{C} / \mathrm{S})+(\mathrm{T})$ & $.0418(2.89)$ & $.0418(2.89)$ & 1.64 & -.010 & 1.29 & -.363 \\
\hline 18 & & & one vehicle & {$[.0448(1.19)]$} & {$[.00242(.306)]$} & & & & \\
\hline 19 & & & $(\mathrm{C} / \mathrm{S})+(\mathrm{C} / \mathrm{S})$ & $.114(2.53)$ & $.0273(5.40)$ & 4.23 & -.009 & .775 & -.240 \\
\hline 20 & & $\stackrel{\pi}{=}$ & $(\mathrm{C} / \mathrm{S})+(\mathrm{T})$ & {$[-.0733(-.667)]$} & $.0676(3.17)$ & & & 2.11 & -.597 \\
\hline 21 & $\vec{d}$ & & one vehicle & {$[.0218(-.800)]$} & {$[-.00128(-.244)]$} & & & & \\
\hline 22 & $\frac{0}{5}$ & & $(\mathrm{C} / \mathrm{S})+(\mathrm{C} / \mathrm{S})$ & $.0938(2.76)$ & $.0304(11.8)$ & 3.14 & -.003 & .785 & -.232 \\
\hline $23 a$ & & & $(\mathrm{C})+(\mathrm{T})$ & .0469 (3.99) & .0469 (3.99) & 1.58 & -.003 & 1.30 & -.290 \\
\hline $23 \mathrm{~b}$ & ن & | & $(\mathrm{S})+(\mathrm{T})$ & $.0640(4.41)$ & $.0640(4.41)$ & 2.15 & -.005 & 1.82 & -.329 \\
\hline 24 & & & one vehicle & {$[.0132(.789)]$} & $-.000526(-.136)$ & & & & \\
\hline 25 & & & $(\mathrm{C} / \mathrm{S})+(\mathrm{C} / \mathrm{S})$ & $.340(2.48)$ & $.0409(4.54)$ & 17.0 & -.212 & 1.39 & -.685 \\
\hline 26 & & $\frac{\pi}{3}$ & $(\mathrm{C} / \mathrm{S})+(\mathrm{T})$ & {$[-.00980(-.271)]$} & $.0720(3.02)$ & & & 2.37 & -1.38 \\
\hline 27 & $\frac{9}{5}$ & & one vehicle & {$[-.00249(-.075)]$} & {$[.00304(.275)]$} & & & & \\
\hline 28 & ㅎำ & & $(\mathrm{C} / \mathrm{S})+(\mathrm{C} / \mathrm{S})$ & $.0263(5.76)$ & $.0263(5.76)$ & 1.04 & -.001 & .779 & -.265 \\
\hline 29 & 包罗 & 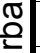 & $(\mathrm{C} / \mathrm{S})+(\mathrm{T})$ & $.0307(2.52)$ & $.0307(2.52)$ & 1.26 & -.002 & .986 & -.278 \\
\hline 30 & & 5 & one vehicle & {$[.00230(.055)]$} & {$[-.0139(-1.37)]$} & & & & \\
\hline
\end{tabular}

* "C", "S" and "T" mean car, SUV and truck respectively.

17 Refer to Equations (2.4) and (2.9), where outcomes "1", "2", "3" correspond to "fatality", "injury", "PDO". We report statistically significant coefficients of the speed limit variable and the corresponding elasticities. In the square brackets we report insignificant coefficients (obtained by test-adding the speed limit variable into the AIC optimal models). All coefficients are the components of vectors $\boldsymbol{\beta}_{1}$ and $\boldsymbol{\beta}_{2}$, multiplied by the speed limit variable in Equation (2.4). 
Table 5.2 2006 accident severity models: results for speed limit

\begin{tabular}{|c|c|c|c|c|c|c|c|c|}
\hline \multirow[b]{2}{*}{ \# } & \multirow{2}{*}{\multicolumn{2}{|c|}{ Model name* }} & \multicolumn{2}{|c|}{$\begin{array}{c}\text { Speed limit } \\
\text { coefficient (t-ratio) } \\
\end{array}$} & \multicolumn{4}{|c|}{$\begin{array}{l}\text { Averaged elasticities of } \\
\text { speed limit (SL) }\end{array}$} \\
\hline & & & fatality $\left[\boldsymbol{\beta}_{1}\right]$ & injury $\left[\boldsymbol{\beta}_{2}\right]$ & $\bar{E}_{1, S L}^{(1)}$ & $\begin{array}{l}\bar{E}_{1 ; ; L}^{(2)}= \\
=\bar{E}_{1 ; S L}^{(3)}\end{array}$ & $\bar{E}_{2 ; S L}^{(2)}$ & $\begin{array}{l}\bar{E}_{2 ; S L}^{(1)}= \\
=\bar{E}_{2 ; S L}^{(3)}\end{array}$ \\
\hline 1 & & $=(\mathrm{C} / \mathrm{S})+(\mathrm{C} / \mathrm{S})$ & $.0396(5.48)$ & $.0396(5.48)$ & 1.61 & -.016 & 1.20 & -.429 \\
\hline 2 & & $=(\mathrm{C} / \mathrm{S})+(\mathrm{T})$ & $.0648(3.06)$ & $.0648(3.06)$ & 2.77 & -.032 & 2.35 & -.453 \\
\hline 3 & శ్ & one vehicle & $.00506(2.04)$ & $.00506(2.04)$ & .235 & -.002 & .185 & -.052 \\
\hline $4 a$ & 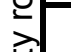 & $(\mathrm{C})+(\mathrm{C})$ & {$[.00689(.000)]$} & {$[.00507(.321)]$} & & & & \\
\hline $4 \mathrm{~b}$ & & $=(\mathrm{C})+(\mathrm{S})$ & {$[.0231(.000)]$} & $.0613(2.43)$ & & & 1.80 & -.405 \\
\hline $4 \mathrm{c}$ & & $(S)+(S)$ & {$[.0110(.000)]$} & {$[.0269(.843)]$} & & & & \\
\hline 5 & & $(\mathrm{C} / \mathrm{S})+(\mathrm{T})$ & {$[-.5454(-.518)]$} & {$[-.0288(-.301)]$} & & & & \\
\hline 6 & & one vehicle & {$[-.0852(-1.23)]$} & {$[.000725(.069)]$} & & & & \\
\hline 7 & & $(\mathrm{C} / \mathrm{S})+(\mathrm{C} / \mathrm{S})$ & {$[.103(1.28)]$} & {$[.00872(.884)]$} & & & & \\
\hline 8 & & $(\mathrm{C} / \mathrm{S})+(\mathrm{T})$ & {$[.150(.908)]$} & {$[.00133(.063)]$} & & & & \\
\hline 9 & $\frac{\Phi}{\omega}$ & one vehicle & {$[-.0237(-1.55)]$} & {$[-.0237(-1.55)]$} & & & & \\
\hline$\overline{10}$ & 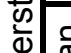 & $(\mathrm{C} / \mathrm{S})+(\mathrm{C} / \mathrm{S})$ & {$[11.04(.000)]$} & {$[-.00108(-.135)]$} & & & & \\
\hline 11 & $\stackrel{\Xi}{\Xi} \mid \frac{\pi}{\xi}$ & $(\mathrm{C} / \mathrm{S})+(\mathrm{T})$ & {$[-.00188(-.011)]$} & {$[.0120(.519)]$} & & & & \\
\hline 12 & & one vehicle & {$[.00776(.197)]$} & {$[.00384(.476)]$} & & & & \\
\hline 13 & & $=(\mathrm{C} / \mathrm{S})+(\mathrm{C} / \mathrm{S})$ & $.248(3.48)$ & $.0416(3.25)$ & 11.9 & -.495 & 1.32 & -.759 \\
\hline 14 & & $(\mathrm{C} / \mathrm{S})+(\mathrm{T})$ & $.127(2.50)$ & $.127(2.50)$ & 5.79 & -.541 & 5.36 & -.970 \\
\hline 15 & วั้ & one vehicle & $0.0636(2.34)$ & {$[.0127(2.25)]$} & 3.34 & -.029 & & \\
\hline 16 & बृ & $(\mathrm{C} / \mathrm{S})+(\mathrm{C} / \mathrm{S})$ & $.251(3.35)$ & $.0290(7.95)$ & 9.40 & -.015 & .835 & -.253 \\
\hline 17 & 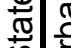 & $(\mathrm{C} / \mathrm{S})+(\mathrm{T})$ & {$[5.60(.000)]$} & {$[.452(1.73)]$} & & & & \\
\hline 18 & & one vehicle & {$[.0268(.418)]$} & {$[-.0115(-.827)]$} & & & & \\
\hline 19 & & $=(\mathrm{C} / \mathrm{S})+(\mathrm{C} / \mathrm{S})$ & $.0414(6.13)$ & $.0414(6.13)$ & 1.46 & -.001 & 1.12 & -.349 \\
\hline 20 & $\cong$ & $(\mathrm{C} / \mathrm{S})+(\mathrm{T})$ & {$[.0185(.000)]$} & {$[.540(1.43)]$} & & & & \\
\hline 21 & & one vehicle & {$[-.0800(-1.46)]$} & {$[-.00409(-.381)]$} & & & & \\
\hline $22 \mathrm{a}$ & 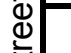 & $(\mathrm{C})+(\mathrm{C})$ & $.0251(6.33)$ & $.0251(6.33)$ & .810 & .000 & .626 & -.184 \\
\hline $22 \mathrm{~b}$ & क्ञा & $(\mathrm{C})+(\mathrm{S})$ & $.0218(4.65)$ & $.0218(4.65)$ & .727 & -.001 & .560 & -.167 \\
\hline $22 \mathrm{c}$ & 表文 & $(S)+(S)$ & $.0343(4.20)$ & $.0343(4.20)$ & 1.14 & -.001 & .865 & -.279 \\
\hline 23 & & $(\mathrm{C} / \mathrm{S})+(\mathrm{T})$ & $.0284(2.34)$ & $.0284(2.34)$ & .937 & -.001 & 831 & -.107 \\
\hline 24 & & one vehicle & {$[.00968(.382)]$} & {$[-.00128(-.240)]$} & & & & \\
\hline 25 & & $=(\mathrm{C} / \mathrm{S})+(\mathrm{C} / \mathrm{S})$ & {$[.0644(1.32)]$} & {$[.0272(1.84)]$} & & & & \\
\hline 26 & & $(\mathrm{C} / \mathrm{S})+(\mathrm{T})$ & $.0608(3.07)$ & $.0608(3.07)$ & 3.12 & -.078 & 2.28 & -.912 \\
\hline 27 & 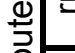 & one vehicle & {$[.0137(1.56)]$} & {$[.0137(1.56)]$} & & & & \\
\hline 28 & 흳 & $(\mathrm{C} / \mathrm{S})+(\mathrm{C} / \mathrm{S})$ & $.0154(2.14)$ & $.0154(2.14)$ & .613 & -.001 & .443 & -.171 \\
\hline 29 & 包) & $(\mathrm{C} / \mathrm{S})+(\mathrm{T})$ & $.0586(3.60)$ & $.0586(3.60)$ & 2.33 & -.027 & 2.02 & -.337 \\
\hline 30 & {$\left[\begin{array}{l}5 \\
\end{array}\right.$} & one vehicle & {$[.0327(.450)]$} & {$[.0134(.878)]$} & & & & \\
\hline
\end{tabular}

* "C", "S" and "T" mean car, SUV and truck respectively. 
Table 5.3 Speed limit effect on structure of 2004 accident severity models ${ }^{18}$

\begin{tabular}{|c|c|c|c|c|c|c|c|c|c|c|}
\hline \# & & & Model name & $M$ & K & $L L\left(\boldsymbol{\beta}_{\mathbf{m}}\right)$ & $\sum L L\left(\boldsymbol{\beta}_{\mathbf{m}}\right)$ & df & $p$-value & conclusion* \\
\hline 1 & \multirow{3}{*}{\multicolumn{2}{|c|}{ 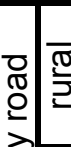 }} & (car/SUV)+(car/SUV) & 6 & 15 & -1656.7 & -1588.2 & 75 & $1.7 e-5$ & SL effect \\
\hline 2 & & & (car/SUV)+(truck) & 5 & 8 & -256.74 & -235.02 & 32 & 0.085 & \\
\hline 3 & & & one vehicle & 7 & 20 & -5066.7 & -4990.7 & 120 & 0.026 & SL effect \\
\hline 4 & \multirow{3}{*}{ క్ } & \multirow{3}{*}{ 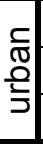 } & $($ car/SUV)+(car/SUV) & 7 & 9 & -691.21 & -657.69 & 54 & 0.11 & \\
\hline 5 & & & (car/SUV)+(truck) & 3 & 5 & -90.86 & -86.40 & 10 & 0.54 & \\
\hline 6 & & & one vehicle & 4 & 8 & -332.07 & -320.00 & 24 & 0.45 & \\
\hline 7 & & \multirow{4}{*}{ 党 } & (car/SUV)+(car/SUV) & 4 & 5 & -414.78 & -404.68 & 15 & 0.16 & \\
\hline $8 a^{* *}$ & & & (car)+(truck) & 3 & 5 & -84.02 & -79.32 & 10 & 0.49 & \\
\hline $8 b$ & $\frac{0}{\pi}$ & & (SUV)+(truck) & 2 & 8 & -49.65 & -45.94 & 8 & 0.49 & \\
\hline 9 & $\frac{\pi}{0}$ & & one vehicle & 4 & 11 & -1346.2 & -1324.2 & 33 & 0.17 & \\
\hline 10 & 苋 & \multirow{3}{*}{$\begin{array}{l}\frac{\mathrm{N}}{\mathrm{N}} \\
\frac{\mathrm{O}}{\mathrm{J}}\end{array}$} & (car/SUV)+(car/SUV) & 3 & 13 & -684.32 & -666.77 & 26 & 0.11 & \\
\hline 11 & & & (car/SUV)+(truck) & 2 & 6 & -299.29 & -296.98 & 6 & 0.60 & \\
\hline 12 & & & one vehicle & 5 & 12 & -761.47 & -738.41 & 48 & 0.55 & \\
\hline 13 & & \multirow{3}{*}{ 준 } & (car/SUV)+(car/SUV) & 2 & 11 & -1310.1 & -1293.1 & 11 & $3.8 \mathrm{e}-4$ & SL effect \\
\hline $14^{\star * *}$ & & & (car/SUV)+(truck) & 4 & 10 & -381.87 & -368.11 & 30 & 0.60 & \\
\hline 15 & $\Phi$ & & one vehicle & 6 & 13 & -2153.5 & -2117.0 & 65 & 0.23 & \\
\hline $16 a$ & 의 & \multirow{3}{*}{ 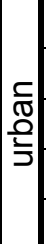 } & (car)+(car) & 8 & 7 & -1129.5 & -1094.6 & 49 & 0.026 & SL effect \\
\hline $16 \mathrm{~b}$ & $\stackrel{\oplus}{\oplus}$ & & (car)+(SUV) & 7 & 10 & -1557.2 & -1512.4 & 60 & $7.8 \mathrm{e}-3$ & SL effect \\
\hline $16 \mathrm{c}$ & 心) & & $(\mathrm{SUV})+(\mathrm{SUV})$ & 3 & 8 & -497.85 & -485.13 & 16 & 0.063 & \\
\hline 17 & & & (car/SUV)+(truck) & 6 & 4 & -177.67 & -162.33 & 20 & 0.059 & \\
\hline 18 & & & one vehicle & 7 & 10 & -618.56 & -569.55 & 60 & $1.4 \mathrm{e}-3$ & SL effect \\
\hline 19 & & \multirow{3}{*}{ 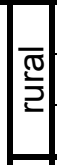 } & $(\mathrm{car} / \mathrm{SUV})+(\mathrm{car} / \mathrm{SUV})$ & 10 & 10 & -1376.0 & -1313.4 & 90 & $8.3 e-3$ & SL effect \\
\hline 20 & & & (car/SUV)+(truck) & 4 & 8 & -73.63 & -63.33 & 24 & 0.66 & \\
\hline 21 & 㐫 & & one vehicle & 6 & 13 & -946.00 & -905.80 & 65 & 0.095 & \\
\hline 22 & के & \multirow{4}{*}{\begin{tabular}{|l} 
\\
$\frac{\pi}{\sqrt{2}}$ \\
$\frac{0}{3}$
\end{tabular}} & $(\mathrm{car} / \mathrm{SUV})+(\mathrm{car} / \mathrm{SUV})$ & 10 & 20 & -14620 & -14435 & 180 & 3.3e-14 & SL effect \\
\hline $23 a$ & 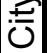 & & (car)+(truck) & 4 & 12 & -534.10 & -509.25 & 36 & 0.064 & \\
\hline $23 b$ & & & (SUV)+(truck) & 6 & 6 & -354.56 & -323.86 & 30 & $6.2 e-4$ & SL effect \\
\hline 24 & & & one vehicle & 5 & 22 & -3627.5 & -3560.3 & 88 & $1.0 \mathrm{e}-3$ & SL effect \\
\hline 25 & \multirow{3}{*}{\multicolumn{2}{|c|}{ 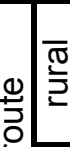 }} & (car/SUV)+(car/SUV) & 6 & 8 & -996.60 & -969.57 & 40 & 0.068 & \\
\hline 26 & & & (car/SUV)+(truck) & 2 & 11 & -275.63 & -262.02 & 11 & $4.3 e-3$ & SL effect \\
\hline 27 & & & one vehicle & 5 & 10 & -1236.7 & -1219.5 & 40 & 0.72 & \\
\hline 28 & $\infty$ & \multirow{3}{*}{$\begin{array}{l}\frac{c}{\mathbb{d}} \\
\frac{0}{丂} \\
\frac{0}{J}\end{array}$} & $(\mathrm{car} / \mathrm{SUV})+(\mathrm{car} / \mathrm{SUV})$ & 5 & 11 & -2361.9 & -2326.9 & 44 & $7.6 e-3$ & SL effect \\
\hline 29 & & & (car/SUV)+(truck) & 7 & 8 & -314.86 & -287.88 & 48 & 0.26 & \\
\hline 30 & & & one vehicle & 7 & 10 & -493.83 & -458.36 & 60 & 0.16 & \\
\hline
\end{tabular}

* - For models with "SL effect" conclusion speed limit is statistically significant for the structure of accident severity models, it is not significant otherwise.

** - Variables X33f and X15 are constant inside speed limit bins and have been removed from the best final logit model before carrying out the test.

${ }^{* * *}$ - Variable X33f is constant and has been removed from the best final logit model.

${ }^{18}$ The tests of the effect are done by using the likelihood ratio, refer to paragraph 3 on page 44 . 
Table 5.4 Speed limit effect on structure of 2006 accident severity models

\begin{tabular}{|c|c|c|c|c|c|c|c|c|c|c|}
\hline \# & & & Model name & $M$ & K & $L L\left(\boldsymbol{\beta}_{\mathbf{m}}\right)$ & $\sum L L\left(\boldsymbol{\beta}_{\mathbf{m}}\right)$ & df & $\mathrm{p}$-value & conclusion* \\
\hline 1 & \multirow{8}{*}{$\frac{\nabla}{\pi}$} & \multirow{3}{*}{\begin{tabular}{l} 
줄 \\
\multirow{2}{2}{}
\end{tabular}} & (car/SUV)+(car/SUV) & 4 & 9 & -847.91 & -813.01 & 27 & $1.2 \mathrm{e}-4$ & SL effect \\
\hline 2 & & & (car/SUV)+(truck) & 5 & 7 & -119.31 & -100.96 & 28 & 0.13 & \\
\hline 3 & & & one vehicle & 5 & 19 & -7154.2 & -7106.0 & 76 & 0.058 & \\
\hline $4 a$ & & \multirow{5}{*}{ 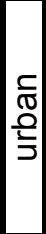 } & $($ car $)+($ car $)$ & 7 & 4 & -303.42 & -291.38 & 24 & 0.46 & \\
\hline $4 b$ & & & (car)+(SUV) & 4 & 3 & -235.35 & -226.24 & 9 & 0.033 & SL effect \\
\hline $4 c$ & & & (SUV)+(SUV) & 4 & 5 & -161.81 & -151.13 & 15 & 0.13 & \\
\hline 5 & & & (car/SUV)+(truck) & 3 & 4 & -15.658 & -98.097 & 8 & 0.17 & \\
\hline 6 & & & one vehicle & 5 & 8 & -342.22 & -330.11 & 32 & 0.84 & \\
\hline 7 & \multirow{6}{*}{ S } & \multirow{3}{*}{$\begin{array}{l}\overline{\widetilde{T}} \\
\stackrel{2}{2}\end{array}$} & (car/SUV)+(car/SUV) & 4 & 9 & -451.87 & -438.45 & 27 & 0.47 & \\
\hline 8 & & & (car/SUV)+(truck) & 2 & 6 & -98.695 & -97.460 & 6 & 0.87 & \\
\hline 9 & & & one vehicle & 5 & 12 & -1474.1 & -1465.6 & 48 & 0.53 & \\
\hline 10 & & \multirow{3}{*}{ 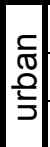 } & (car/SUV)+(car/SUV) & 8 & 9 & -836.55 & -811.72 & 63 & 0.89 & \\
\hline 11 & & & (car/SUV)+(truck) & 2 & 9 & -221.17 & -215.30 & 9 & 0.23 & \\
\hline 12 & & & one vehicle & 5 & 11 & -882.75 & -862.84 & 44 & 0.65 & \\
\hline 13 & \multirow{3}{*}{\multicolumn{2}{|c|}{ 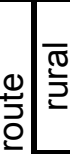 }} & (car/SUV)+(car/SUV) & 2 & 9 & -575.69 & -559.57 & 9 & $1.8 e-4$ & SL effect \\
\hline 14 & & & (car/SUV)+(truck) & 2 & 4 & -76.769 & -72.761 & 4 & 0.091 & \\
\hline 15 & & & one vehicle & 5 & 16 & -3707.8 & -3657.2 & 64 & 0.00204 & SL effect \\
\hline 16 & \multirow{3}{*}{$\begin{array}{l}\bar{\Phi} \\
\stackrel{\mathbb{N}}{\infty}\end{array}$} & \multirow{3}{*}{ 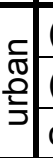 } & (car/SUV)+(car/SUV) & 8 & 10 & -3268.3 & -3203.0 & 70 & $1.5 e-4$ & SL effect \\
\hline 17 & & & (car/SUV)+(truck) & 5 & 5 & -153.32 & -146.63 & 20 & 0.86 & \\
\hline 18 & & & one vehicle & 6 & 8 & -747.88 & -715.34 & 40 & $7.4 \mathrm{e}-3$ & SL effect \\
\hline 19 & \multirow{8}{*}{ 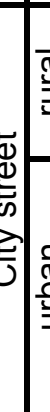 } & \multirow{3}{*}{ 잔. } & (car/SUV)+(car/SUV) & 7 & 7 & -1088.1 & -1046.1 & 42 & $1.3 e-3$ & SL effect \\
\hline 20 & & & (car/SUV)+(truck) & 3 & 2 & -2851.8 & -2834.5 & 4 & $5.3 e-6$ & SL effect \\
\hline 21 & & & one vehicle & 2 & 11 & -288.38 & -283.71 & 11 & 0.59 & \\
\hline $22 a$ & & \multirow{5}{*}{ 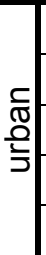 } & (car)+(car) & 6 & 15 & -6848.4 & -6782.1 & 75 & $4.8 e-4$ & SL effect \\
\hline $22 b$ & & & (car)+(SUV) & 6 & 14 & -4300.4 & -4247.2 & 70 & $3.3 e-2$ & SL effect \\
\hline $22 c$ & & & $($ SUV)+(SUV) & 2 & 10 & -1350.6 & -1338.5 & 10 & $7.4 \mathrm{e}-2$ & SL effect \\
\hline 23 & & & (car/SUV)+(truck) & 6 & 11 & -523.35 & -498.99 & 55 & 0.71 & \\
\hline 24 & & & one vehicle & 4 & 20 & -2432.0 & -2393.4 & 60 & 0.065 & \\
\hline 25 & \multirow{3}{*}{\multicolumn{2}{|c|}{ 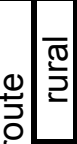 }} & (car/SUV)+(car/SUV) & 4 & 9 & -329.22 & -311.62 & 27 & 0.13 & \\
\hline 26 & & & (car/SUV)+(truck) & 3 & 7 & -243.30 & -233.82 & 14 & 0.17 & \\
\hline 27 & & & one vehicle & 2 & 14 & -1484.2 & 1472.3 & 14 & 0.049 & SL effect \\
\hline 28 & \multirow{3}{*}{\multicolumn{2}{|c|}{$\begin{array}{l}\frac{c}{2} \\
\frac{8}{3}\end{array}$}} & (car/SUV)+(car/SUV) & 2 & 7 & -969.51 & -961.10 & 7 & 0.019 & SL effect \\
\hline 29 & & & (car/SUV)+(truck) & 2 & 6 & -192.74 & -184.75 & 6 & 0.014 & SL effect \\
\hline 30 & & & one vehicle & 3 & 9 & -173.27 & -166.32 & 18 & 0.74 & \\
\hline
\end{tabular}

* - For models with "SL effect" conclusion speed limit is statistically significant for the structure of accident severity models, it is not significant otherwise. 
Next let us refer to Table 5.3 and Table 5.4, which are related to 2004 and 2006 accident data respectively. These tables give the results for the log-likelihood ratio tests of statistically significant differences among the models estimated for different speed-limit bins for each of the considered road-class-accident-type combination (the bins themselves are given in Table C.3 and Table C. 4 in Appendix $\mathrm{C}$ ). We find the following results:

- Speed limit does not have any statistically significant effect on the structure of severity models for 2004 and 2006 accidents on interstate highways (both urban and rural).

- Speed limit has a statistically significant effect on the structure of severity models for accidents on all other road classes (with the exception of 2004 accidents on urban county maintained roads, for which speed limit has no any statistically significant effect on the severity model structure).

\subsubsection{Effect of Other Explanatory Variables}

Now we use model estimation results given in Table C.5 and Table C. 6 in Appendix $\mathrm{C}$ and consider the influence of explanatory variables other than the posted speed limit on severity of 2004 and 2006 accidents. Similar to the accident causation study, we again limit our consideration to several of the most important and most statistically significant variables.

- Variables "wint" and "sum" (see pages 113 and 143): We find that the probability of higher severity of an accident generally decreases during winters and increases during summers (this effect was stronger is year 2004). This result appears to be an unexpected result. However, it can be explained. First, drivers do take some extra precautions during bad winter weather. Although these precautions are not sufficient to keep the drivers safe (refer to accident causation study results in Section 4.2.2), they can reduce probabilities of very severe accident outcomes. 
Second, it is very likely that the number of minor (PDO) accidents sharply increases during winters due to less safe weather and roadway conditions. This increase shifts the outcome probabilities (conditioned on the fact that an accident occurred and observed) toward less severe accident outcomes. In other words, the number of serious accidents (e.g. fatalities) might increase during winters, but the number of minor accidents is likely to increase much more. The summer seasonal effect is the opposite of the winter seasonal effect.

- Variables "dark" and "darklamp" (see pages 118, 119, 148 and 149): We find that the probability of higher severity of an accident generally increases when the road is dark, even if there are street lights. The explanation can be that during night time drivers have harder time controlling their vehicles and holding the road. Thus, drivers become more likely to be involved into serious accidents (such as head-on collisions, collisions with stationary objects, and rollovers).

- Variables "nojun" and "way4" (see pages 123, 153 and 154): We find that accidents are generally more likely to be more severe if they occur at road junctions, and especially at 4-way intersections. This effect mainly concerns two-vehicle accidents and it can be explained as follows. On one hand, two-vehicle collisions in which one vehicle hits a side of the other vehicle are most likely to occur at road junctions. On the other hand, side impacts are highly dangerous due to high driver and passenger vulnerability during such impacts.

- Variables "driver" and "env" (see pages 127, 128 and 155): We find that the probability of higher severity of an accident generally increases when the primary cause of the accident is driver-related, while the probability decreases when the primary cause is environment-related. This is a relatively strong effect. It is due to human mistakes being especially dangerous because they are totally unpredictable, while 
environment (e.g. weather-related) factors are observable and can be accounted for by taking additional precautions.

- Variables "hl5", "hl10" and "hl20" (see pages 128, 129, 156 \& 157): Depending on the road class and accident type, we find that an accident has higher probability of a severe outcome if help arrives more quickly. This effect can be due to a data selection bias because help is not needed at all in the case of minor accidents.

- Variable "moto" (see pages 130 and 157): Accidents involving a motorcycle are typically more severe. This is a strong effect. It is explained by very high vulnerability of motorcycle riders.

- Variable "vage" and "voldo" (see pages 132, 140, 158 and 165): We find that the probability of higher severity of an accident increases with the age of a vehicle involved into the accident. This may be an outgrowth of older vehicles being generally less safe than newer vehicles.

- Variable " $X_{27}$ " and "maxpass" (see pages 133, 140, 159 and 166): We find that the probability of higher severity of an accident typically increases with the number of occupants in vehicles involved into the accident. This effect is expected for three reasons. First, the likelihoods of at least one death and at least one injury increase when there are more occupants in a colliding vehicle. Second, occupants' bodies hit each other during a collision. Third, a more heavily occupied vehicle has higher mass and higher kinetic energy to dissipate during a collision.

- Variable "priv" (see pages 135 and 160): We find that accidents occurred in private drives are typically less severe. Such accidents are minor because vehicles generally travel at low speeds in private drives.

- Variable " $X_{33}$ " (see pages 137 and 163): We find that the probability of higher severity of an accident considerably increases when at least one of the vehicles involved in the accident caught fire. This is a very strong effect. Obviously, fire is very dangerous for drivers and passengers involved into an accident. 
- Variables " $X_{35}$ ", "ff" and "mm" (see pages 139, 141, 165 and 168): We find that generally the probability of higher severity of an accident increases when the driver at fault is female. In addition, when two female drivers are involved in a two-vehicle accident, then this accident is more likely to be reported as severe, as compared to the case when two male drivers are involved. This could be the result of physical and behavioral difference between genders.

The previous analysis is based on cross-sectional data comparing the effect of different speed limits on a class of roadways for a single year. This is an important consideration because it controls for possible changes in vehicle safety features, enforcement levels and driver behavior that may change from one year to the next. Still, it may also be of interest to compare 2004 and 2006 accident-severity models to see if the 2005 speed-limit increase (and the other factors that may have varied over this time period) significantly changed the estimated parameters in the accident severity models. To address this possibility, we consider; 1) interstates that remained had the same $55 \mathrm{mph}$ speed limits in 2004 and 2006 (which are in urban areas), and 2) interstates that were $65 \mathrm{mph}$ in 2004 and increased to $70 \mathrm{mph}$ in 2006 (which are in rural areas). The appropriate statistical test is (Washington et. al, 2003),

$$
-2\left[L L\left(\boldsymbol{\beta}_{\text {all }}\right)-L L\left(\boldsymbol{\beta}_{2004}\right)-L L\left(\boldsymbol{\beta}_{2006}\right)\right] \sim \chi_{d f=K}^{2}, \quad \text { Eq. } 5.1
$$

where $L L\left(\boldsymbol{\beta}_{\text {all }}\right)$ is the log-likelihood at converged values of $\boldsymbol{\beta}$ for the model estimated 2004 and 2006 data for the accident type being considered; $L L\left(\boldsymbol{\beta}_{2004}\right)$ is the log-likelihood of the model estimated for 2004 observations; $\operatorname{LL}\left(\boldsymbol{\beta}_{2006}\right)$ is the log-likelihood of the model estimated for 2006 observations; and $K$ is the number of parameters estimated for each model.

The estimation results show that the accident severity parameter estimates (for single and multivehicle crashes) did not significantly change from 2004 to 2006 
on Interstates that remained at $55 \mathrm{mph}$. This indicates that the "halo effect" of the increased speed limits (from $65 \mathrm{mph}$ to $70 \mathrm{mph}$ in rural areas) did not significantly affect those interstates that remained at $55 \mathrm{mph}$. Temporal stability tests also showed that accident severity parameter estimates (for single and multivehicle crashes) did not significantly change from 2004 to 2006 on Interstates that were $65 \mathrm{mph}$ in 2004 and either 65 or $70 \mathrm{mph}$ in 2006 . The temporal stability of these models adds further evidence to support the crosssectional finding that the higher range of speed limits in effect on Indiana interstates in 2006 has not significantly affected the severity of accidents. 


\section{CHAPTER 6. SPEED LIMITS AND SPEED-LIMIT COMPLIANCE}

In this chapter we will not focus on the accident-generating outcomes of increased speed limits, but instead try to provide insight into the factors that determine drivers' usual speed selection when faced with specific speed limits under low traffic-volume conditions (free-flow conditions as defined by the Transportation Research Board, 2000, see also Mannering et. al., 2005). To be sure, previous studies have looked at factors that affect usual driving speeds (Kweon and Kockelman, 2003). However, this chapter differs from previous work in that it considers normally selected driving speeds under a range of speed limits that concurrently exist on interstate highways in the study area. By considering such a range of speed limits on drivers' normally selected speed, it is hoped that some additional insight can be provided with regard to the tradeoffs drivers make with regard to speed, safety and the adherence to speed-limit laws.

\subsection{Effect of Speed Limits on Drivers' Choice of Speed}

To understand the effects that speed limits have on drivers' speed choice, some of the principles developed in previous literature relating to risk selection are used (Peltzman, 1975; Viscusi and Carvallo, 1995; Winston et. al., 2006). In so doing, drivers are assumed to maximize their utility from driving by trading off driving speed and safety. Figure 6.1 shows how speed limits can affect the tradeoff between drivers' choice of driving speed and safety. For illustrative purposes, consider safety as the probability of avoiding an accident. Following Winston, Maheshri and Mannering (2006) in Figure 6.1, the marginal rate of 
transformation between safety $S$ and driving speed $S$ is shown by the slope of line Ss (linearity is assumed for simplicity). Given this marginal rate of transformation between safety and speed, and the absence of a speed limit, the driver maximizes utility at equilibrium $A$ (reflecting the tangency of an indifference curve $U_{0}$ ), with $S^{*}$ and $s^{*}$ chosen as the optimal levels of safety and driving speed. In the presence of a speed limit below $A$ that is strictly followed, the trade-off between accident likelihood and speed will move to $B$. However, since $B$ is below drivers' optimal speed selection, drivers will tend toward their optimal speed/safety equilibrium (point $A$ ). They trade off the additional utility of driving faster against the risk of detection by law enforcement, their respect for the law, and so on, resulting in a new equilibrium at point $C$, which is likely to be somewhere between points $A$ and $B$ for most drivers.

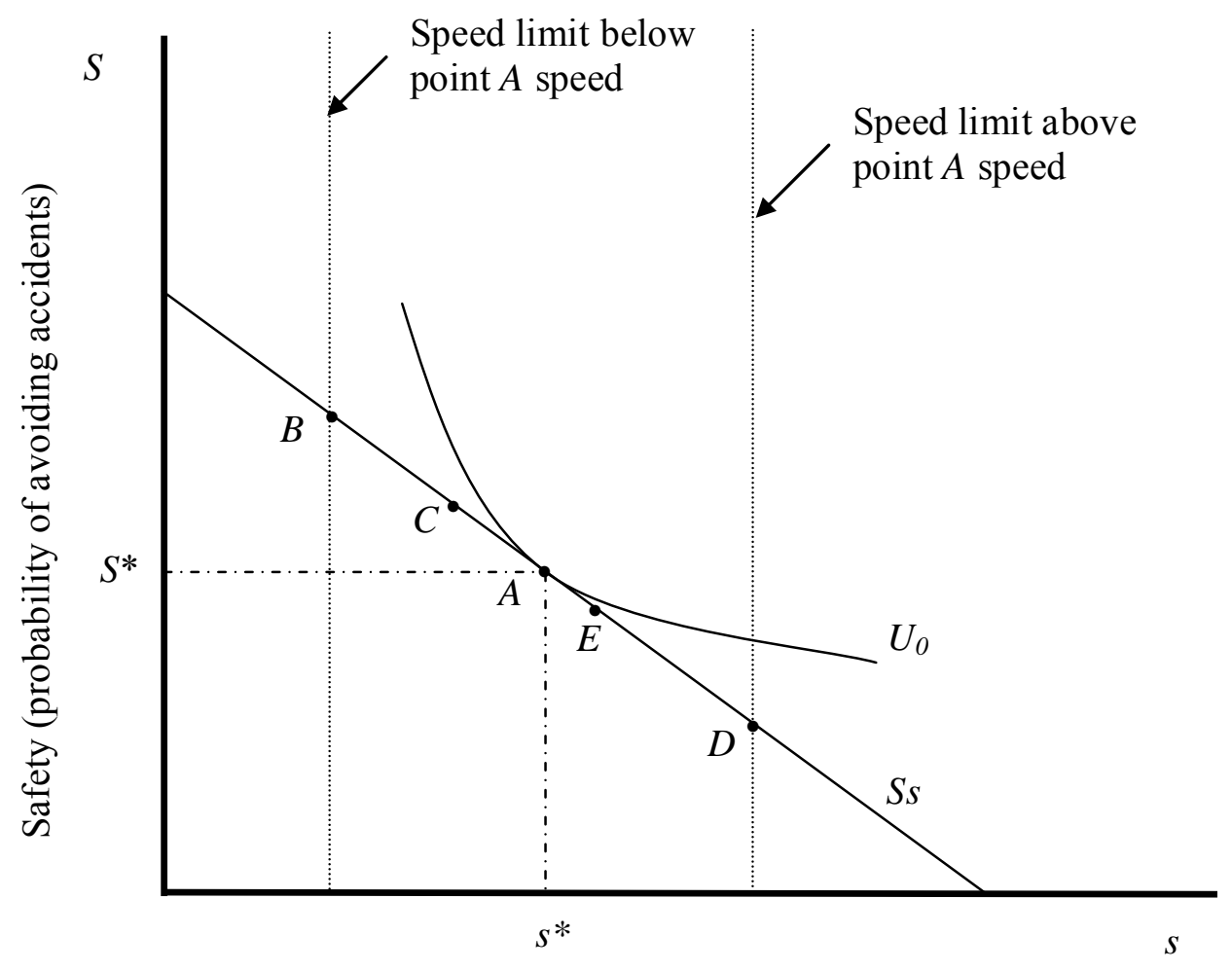

Figure 6.1 The effect of Speed Limits on Driving Speed 
One could also imagine a case where the speed limit is set above point $A$ (this may be the case for some older drivers, for example, that might have lower speed equilibrium points). For these drivers, point $D$ would be too risky. While one would expect these drivers to still choose point $A$, it is possible that they may try to maintain a higher speed and end up at some point to the left of $A$ (such as point $E$ ).

Given the range of driver abilities, values of time (utility of speed) and acceptance of risk, for any given section of highway and traffic flow conditions there will be a distribution of point $A$ 's that will determine the variance of traffic speed. Depending on traffic conditions and driver populations, the imposition of a speed limit will result in new equilibrium points that could increase or decrease this variance.

Please note in the above examples, for purposes of exposition, it is assumed that the slope of Ss will remain constant. In fact, the speed selection choices of other drivers in the presence of speed limits may alter risks and change the slope of Ss.

\subsection{Data}

Given the discussion relating to Figure 6.1, the empirical question becomes one of identifying the factors that determine individual drivers' choice of point $C$ (or point $E$ if speed limits are too high). To study this, a survey was developed and administered to Indiana residents in the late fall of 2005 on the campus of Purdue University. The timing of the survey was particularly relevant since Indiana raised the speed limits on its rural interstates from $65 \mathrm{mph}$ to $70 \mathrm{mph}$ on July 1, 2005. Thus survey respondents had current experience with interstate speed limits set at $55 \mathrm{mph}$ (urban areas), $65 \mathrm{mph}$ (some suburban areas that were not increased to $70 \mathrm{mph}$ ) and $70 \mathrm{mph}$ (rural areas). 
The survey questions are presented in Table 6.1. To avoid the interactions of traffic flow and speed, the speed-related questions (Questions 2, 3 and 4) focused on self-reported normal driving speeds on interstate highways (of specified speed limit) with little traffic. As can be seen from Table 6.1, a broad range of socioeconomic and opinion data were gathered.

The survey was administered to a sample of licensed drivers who indicated they regularly drove on Indiana freeways. The sample consisted of undergraduate and graduate students at Purdue, primarily from engineering disciplines. Because our study is exploratory in nature, no attempt was made to acquire a random sample of Purdue University students (or Indiana drivers). The implication of this will be discussed later in the chapter. In all, 250 surveys were administered (most in a classroom environment), and 204 surveys were returned with complete information. Of these 204 respondents, 194 reported normally driving above a $55 \mathrm{mph}$ speed limit, 190 reported normally driving above a $65 \mathrm{mph}$ speed limit, and 178 reported normally driving above a $70 \mathrm{mph}$ speed limit. This would suggest that for the majority of drivers in the survey, the speed limit is to the left of their point $A$ (see Figure 6.1). However, there is also evidence that a small number of survey respondents view the speed limit as being set above their point $A$. Of the 204 respondents, 1 person normally drove below the $55 \mathrm{mph}$ speed limit (9 reported they drive at the $55 \mathrm{mph}$ speed limit), 3 people normally drove below the $65 \mathrm{mph}$ speed limit (11 reported they drive at the $65 \mathrm{mph}$ speed limit), and 6 people drove below the $70 \mathrm{mph}$ speed limit (20 reported they drive at the $70 \mathrm{mph}$ speed limit).

Summary statistics for the sample are presented in Table 6.2. This table shows that the reported average normal driving speed was nearly $66 \mathrm{mph}$ on interstates posted with $55 \mathrm{mph}$ speed limits, about $74 \mathrm{mph}$ on interstates posted at $65 \mathrm{mph}$ and almost $78 \mathrm{mph}$ on interstates that are posted at $70 \mathrm{mph}$. 
Table 6.1 Survey Questions

1. Indiana recently raised the speed limit on some of its interstate highways from $65 \mathrm{mph}$ to $70 \mathrm{mph}$. Do you think this is:
$\square$ too fast
$\square$ about right
$\square$ still too slow

2. On an interstate with a $55 \mathrm{mph}$ speed limit and little traffic, about how fast do you normally drive? $\mathrm{mph}$

3. On an interstate with a $65 \mathrm{mph}$ speed limit and little traffic, about how fast do you normally drive? $\mathrm{mph}$

4. On an interstate with a $70 \mathrm{mph}$ speed limit and little traffic, about how fast do you normally drive? $\mathrm{mph}$

5. How would you rate the quality of pavements on Indiana interstates?
$\square$ poor $\square$ fair
$\square$ good
$\square$ very good
$\square$ don't know

6. Compared to adjacent states (Ohio, Illinois, Michigan and Kentucky), how would you rate the quality of pavements on Indiana interstates?
$\square$ worse than adjacent states
$\square$ about the same
$\square$ better than adjacent states
$\square$ don't know

7. Which one of these luxury car brands do you believe has the most prestige? (select one)
$\square$ Acura
$\square$ Audi
$\square$ BMW
$\square$ Cadillac $\quad \square$ Infiniti
$\square$ Jaguar
$\square$ Lexus
$\square$ Lincoln
$\square$ Mercedes

8. Which one of these of these vehicle brands do you believe provides the best value for the money? (select one)
$\square$ Chevrolet
$\square$ Dodge
$\square$ Ford
$\square$ Honda
$\square$ Hyundai
$\square$ Mazda
$\square$ Nissan
$\square$ Toyota
$\square$ female
$\square$ male
$\square$ single
$\square$ separated

$$
\square \text { divorced } \square \text { other }
$$

9. Are you?

10. Are you?

11. What is your age?

12. What is your highest completed level of education?
$\square$ some high school
$\square$ technical college degree
$\square$ post graduate degree
$\square$ high school diploma
$\square$ college degree

13. What is the approximate annual household income of the household you consider home?
$\square$ no income
$\square$ under $\$ 10,000$
$\square$ \$10,000-\$19,999
$\square$ \$20,000-\$29,999
$\square \$ 30,000-\$ 39,999$
$\square \$ 40,000-\$ 49,999$
$\square \$ 50,000-\$ 74,999$
$\square \$ 75,000-\$ 100,000$
$\square$ Over $\$ 100,000$

14. Including yourself, how many people live in the household you consider home?

15. How many children, in the household you consider home, are under age 6 ?

16. How many children, in the household you consider home, are aged 6 to 16 ?

17. How many people living, in the household you consider home, work outside the home?

18. How many licensed and operable motor vehicles does your "home" household have?

19. How many years have you had a driver's license? 
Kockelman and Bottom (2006) found, with observed speed data from Austin, Texas, that a $5 \mathrm{mph}$ increase in the speed limit was associated with a $3.2 \mathrm{mph}$ increase in average speeds. In the current sample, the observed $3.83 \mathrm{mph}$ increase in normal speeds between $65 \mathrm{mph}$ and $70 \mathrm{mph}$ posted interstates is close to this.

The data indicate that the amount driven above the speed limit declines with higher speed limits (presumably as the speed limit approaches point $A$ in Figure 6.1). In terms of the standard deviations of normal driving speeds, the greatest reported variance is at the $55 \mathrm{mph}$ speed limit $(6.24 \mathrm{mph})$, and the variances at $65 \mathrm{mph}$ and $70 \mathrm{mph}$ speed limits are roughly the same $(5.03 \mathrm{mph}$ and $5.24 \mathrm{mph}$, respectively). Although the variance differences among the three speed limits are small, which is consistent with the findings of Kockelman and Bottom (2006), there is a higher variance with the $55 \mathrm{mph}$ speed limit. While the sample is small and traffic-related effects are not accounted for (recall that only low-volume conditions are considered), in this sample it appears that a $55 \mathrm{mph}$ speed limit may be too slow to achieve minimum speed variance.

Table 6.2 shows that the values of other variables in the sample, particularly those relating to socioeconomic variables, reflect those of the Purdue University community - with an average age of only $25,25 \%$ being married, $18 \%$ having children under 6 years of age, and so on.

\subsection{Methodology}

As indicated in Table 6.1 and Table 6.2, the survey contains three questions relating to normal driving speed under various speed limits; 1) normal driving speed on an interstate with a $70 \mathrm{mph}$ speed limit and little traffic, 2) normal driving speed on an interstate with a $65 \mathrm{mph}$ speed limit and little traffic, and 3) normal driving speed on an interstate with a $55 \mathrm{mph}$ speed limit and little traffic. 
Table 6.2 Sample statistics (standard deviation in parentheses when appropriate)

Variable Values

Percent believing Indiana's recently raised the speed limits from 2/72/26

$65 \mathrm{mph}$ to $70 \mathrm{mph}$ is:

too fast/about right/still too slow

Average normal driving speed on an interstate with a $55 \mathrm{mph}$

$65.92(6.24)$

speed limit and little traffic

Average normal driving speed on an interstate with a $65 \mathrm{mph}$ speed limit and little traffic

$74.05(5.03)$

Average normal driving speed on an interstate with a $70 \mathrm{mph}$ speed limit and little traffic

Percent rating the quality of pavements on Indiana interstates as: 11/30/44/9/6 poor/fair/good/very good/don't know

Percent rating the quality of pavements on Indiana interstates as: $12 / 45 / 16 / 27$ worse than adjacent states/about the same/better than adjacent states/don't know

Percent believing the following luxury car brands provide the

$1 / 6 / 23 / 4 / 2 / 19 / 10 / 2 / 33$ most prestige:

Acura/ Audi/BMW/Cadillac/Infiniti/Jaguar/Lexus/Lincoln/Mercedes

Benz

Percent believing the following vehicle brands provide the best value for the money:

Chevrolet/Dodge/Ford/Honda/Hyundai/Kia/Mazda/Nissan/Toyota

Percent: female/male

$26 / 74$

Percent: married/single/separated/divorced/other

$25 / 71 / 0 / 0 / 4$

Average age

Percent with highest completed level of education: some high school/ high school diploma/technical college degree/college degree /post graduate degree

Percent with annual household income as: no income/ under $\$ 10,000 / \$ 10,000-\$ 19,999 / \$ 20,000-$ $\$ 29,999 / \$ 30,000-\$ 39,999 / \$ 40,000-\$ 49,999 / \$ 50,000-$ $\$ 74,999 / \$ 75,000-\$ 100,000 /$ Over $\$ 100,000$

Average number of people living in household

Average number of children in household that are under age 6

$0.18(0.50)$

Average number of children in household that are aged 6 to 16

$0.26(0.60)$

Average number of people in household that work outside the home

Average number of licensed motor vehicles in household

Average number of years licensed 
To develop a statistical model for each of these three questions, the use of ordinary least squares regressions is an obvious choice. Under standard linear regression assumptions, that includes the assumption that the model has all of the information relating to the regression equation and variables, estimated regression coefficients for the three equations are unbiased and efficient (Washington et. al., 2003). However, if some information is not taken into account, the properties relating to the unbiasedness and efficiency of estimated coefficients cannot be determined. A classic example of potentially missing information is the knowledge that the disturbance term in one regression equation is correlated with the disturbance term in another. This will be the case for the three equations relating to normal driving speed at different speed limits since the unobserved factors that determine driving speed for each speed limit will likely be highly correlated.

For model formulation, the speed variables are transformed by subtracting the speed limit from the reported normal driving speed - giving the number of miles per hour normally driven above the speed limit. This is formalized in the following equation system:

$$
\begin{aligned}
& \text { Speed }_{70}=\beta_{70} Z+\alpha_{70} X+\varepsilon_{70} \\
& \text { Speed }_{65}=\beta_{65} Z+\alpha_{65} X+\varepsilon_{65} \\
& \text { Speed }_{55}=\beta_{55} Z+\alpha_{55} X+\varepsilon_{55}
\end{aligned}
$$

In these equations, Speed $_{70}$, Speed $_{65}$ and Speed $_{55}$ are the number of miles per hour respondents normally drive above the speed limit (with little traffic) for 70 , 65 , and $55 \mathrm{mph}$ speed limits, respectively. These variables can take on positive values if respondents normally drive above the speed limit and negative values if they normally drive below it. Also in these equations, $Z$ is a vector of driver and driver-household characteristics, $X$ is a vector of vector of driver 
preferences and opinions, $\beta$ s, $\alpha$ 's, are vectors of estimable coefficients, and $\varepsilon$ 's are disturbance terms. Note that Equations 6.1, 6.2 and 6.3 do not directly interact with each other. That is, Speed $_{70}$, does not directly determine Speed $_{65}$, Speed $_{65}$ does not directly affect Speed $_{55}$, and so on, as one would expect in a classic simultaneous equation system (see, for example, Shankar and Mannering, 1998). However, because all three responses represented by Equations 6.1, 6.2 and 6.3 are from the same driver, these equations are likely to share unobserved characteristics. In this case, the equations are seemingly unrelated but there will be contemporaneous (cross-equation) correlation of error terms. If Equations 6.1, 6.2 and 6.3 are estimated separately by ordinary least squares, the coefficient estimates are consistent but not efficient. Efficient coefficient estimates are obtained by considering the contemporaneous correlation of disturbances $\varepsilon_{1}, \varepsilon_{2}$ and $\varepsilon_{3}$. Considering contemporaneous correlation in seemingly unrelated equations was first considered by Zellner (1962). Estimation of seemingly unrelated equations is accomplished using generalized least squares. Recall that under ordinary least squares assumptions the disturbance terms have equal variances and are not correlated, resulting in coefficients being estimated as,

$$
\hat{\beta}=\left(X^{T} X\right)^{-1} X^{T} Y
$$

where $\hat{\beta}$ is a $\mathrm{p} \times 1$ column vector (where $\mathrm{p}$ is the number of coefficients), $\mathrm{X}$ is an $\mathrm{n} \times \mathrm{p}$ matrix of data (where $\mathrm{n}$ is the number of observations), $X^{T}$ is the transpose of $X$, and $Y$ is an $n \times 1$ column vector. Generalized least squares generalizes this expression by using a matrix that considers for correlation among equation error terms $(\Omega)$, Equation 6.4 is rewritten as,

$$
\hat{\beta}=\left(X^{T} \Omega^{-1} X\right)^{-1} X^{T} \Omega^{-1} Y
$$

The most difficult aspect of generalized least squares estimation is obtaining an estimate of the $\Omega$ matrix. In seemingly unrelated regression estimation, $\Omega$ is 
estimated from initial ordinary least squares estimates of individual equations (Washington et. al. 2003).

\subsection{Estimation Results}

Table 6.3 presents the sample statistics for the variables found to be significant in the speed models. Excluding respondents with missing data, there were 195 valid observations. Following the numbers presented earlier in Table 6.2, with regard to the dependent variables, the table shows that drivers reported that they normally drove an average of nearly $11 \mathrm{mph}$ faster than the speed limit on interstates posted $55 \mathrm{mph}$, about $9 \mathrm{mph}$ faster than the speed limit on interstates posted at $65 \mathrm{mph}$, and nearly $8 \mathrm{mph}$ faster than the speed limit on interstates posted at $70 \mathrm{mph}$. The standard deviation of normal driving speeds above the posted speed limit was $6.21 \mathrm{mph}$ for $55 \mathrm{mph}$ speed limits, $5.10 \mathrm{mph}$ for $65 \mathrm{mph}$ speed limits and $5.35 \mathrm{mph}$ for $70 \mathrm{mph}$ speed limits.

Seemingly unrelated regression estimation results are presented in Table 6.4. In general, the models fit the data well (especially considering the variance in the data) and the coefficient estimates are of plausible sign and generally good statistical reliability. It should be noted that these same models were run as separate regressions using ordinary least squares. The ordinary least squares regression coefficients had noticeably higher standard errors resulting in lower $t$ statistics (in fact, three variables included in the models presented in Table 6.4 had $t$-statistics less than one). Thus, the efficiency gains from using seemingly unrelated regression estimation are considerable in this case.

The results in Table 6.4 show that men normally drive faster than the speed limit on interstates posted $65 \mathrm{mph}$. However, this coefficient estimate was not highly significant and the male indicator variable was not statistically significant ( $p$ values less than 0.10 ) on interstates posted $55 \mathrm{mph}$ and $65 \mathrm{mph}$. 
As expected, increasing driver age generally had a negative effect on the miles per hour normally driven above the speed limit. The age variable was significant for interstates posted $65 \mathrm{mph}$ and $70 \mathrm{mph}$, resulting in about a tenth of a mile per hour reduction for each year older the driver becomes. However, age was found to be statistically insignificant for interstates with $55 \mathrm{mph}$ speed limits.

Drivers from high-income households ( $\$ 75,000$ per year or greater) where found to be more likely to normally drive faster under all three speed-limit postings considered. Having a higher income resulted in about $2 \mathrm{mph}$ higher normal speeds for $55 \mathrm{mph}$ and $65 \mathrm{mph}$ speed limits and a little over $1 \mathrm{mph}$ higher speed for interstates with $70 \mathrm{mph}$ speed limits. This could be reflecting their higher value of time or perhaps higher vehicle quality.

Drivers from lower-income households ( $\$ 30,000$ per year or greater) where found to be more likely to normally drive slower on interstates with $55 \mathrm{mph}$ speed limits. However, this variable was not highly significant ( $p$-value of 0.12 ) and the effect on normal speed was less than $1 \mathrm{mph}$. Again, this could be a result of value of time and vehicle quality.

The number of children less than six years of age in the household caused normal driving speeds to be lower for all three speed-limit models. The effect was to lower normal driver speeds about $1 \mathrm{mph}$. This may be a result of higher safety awareness among drivers with younger children. 
Table 6.3 Statistics for variables found to be significant in model estimation

\begin{tabular}{|c|c|c|c|}
\hline Variable & Mean & $\begin{array}{l}\text { Standard } \\
\text { deviation }\end{array}$ & $\begin{array}{l}\text { Minimuml } \\
\text { Maximum }\end{array}$ \\
\hline \multicolumn{4}{|l|}{ Dependent variables } \\
\hline Miles per hour normally driven above a $55 \mathrm{mph}$ speed limit & 10.96 & 6.21 & $-5 / 35$ \\
\hline Miles per hour normally driven above a $65 \mathrm{mph}$ speed limit & 9.06 & 5.10 & $-5 / 25$ \\
\hline Miles per hour normally driven above a $70 \mathrm{mph}$ speed limit & 7.88 & 5.35 & $-10 / 20$ \\
\hline \multicolumn{4}{|l|}{ Driver/household attributes } \\
\hline Male indicator ( 1 if driver is male, 0 otherwise) & 0.742 & 0.439 & $0 / 1$ \\
\hline Driver age (years) & 25.21 & 6.58 & $17 / 51$ \\
\hline $\begin{array}{l}\text { High income indicator ( } 1 \text { if household's total annual income is } \\
\$ 75,000 \text { or greater, } 0 \text { otherwise) }\end{array}$ & 0.268 & 0.444 & $0 / 1$ \\
\hline $\begin{array}{l}\text { Low income indicator ( } 1 \text { if household's total annual income is } \\
\text { less than } \$ 30,000,0 \text { otherwise) }\end{array}$ & 0.289 & 0.454 & $0 / 1$ \\
\hline $\begin{array}{l}\text { Number of children under the age of } 6 \text { years old in the } \\
\text { household }\end{array}$ & 0.196 & 0.522 & $0 / 3$ \\
\hline $\begin{array}{l}\text { Late license indicator ( } 1 \text { if driver was first licensed at age } 17 \text { or } \\
\text { greater, } 0 \text { otherwise) }\end{array}$ & 0.361 & 0.481 & $0 / 1$ \\
\hline \multicolumn{4}{|l|}{ Driver opinions } \\
\hline $\begin{array}{l}\text { Good pavement indicator ( } 1 \text { if driver believes pavement quality } \\
\text { on Indiana interstates is good or very good, } 0 \text { otherwise) }\end{array}$ & 0.531 & 0.500 & $0 / 1$ \\
\hline $\begin{array}{l}\text { German prestige indicator ( } 1 \text { if driver believes if German-brand } \\
\text { vehicles are the most prestigious, } 0 \text { otherwise) }\end{array}$ & 0.634 & 0.483 & $0 / 1$ \\
\hline $\begin{array}{l}\text { Japanese prestige indicator ( } 1 \text { if driver believes if Japanese- } \\
\text { brand vehicles are the most prestigious, } 0 \text { otherwise) }\end{array}$ & 0.119 & 0.324 & $0 / 1$ \\
\hline
\end{tabular}


Table 6.4 Seemingly unrelated regression equation (SURE) estimation results for the number of miles per hour above the speed limit drivers report as their usual speed (t-statistics in parentheses) ${ }^{a}$

\begin{tabular}{|c|c|c|c|}
\hline Variable & $\begin{array}{l}\text { Estimated } \\
\text { Coefficient } \\
\text { (55 mph SL) }\end{array}$ & $\begin{array}{l}\text { Estimated } \\
\text { Coefficient } \\
\text { (65 mph SL) }\end{array}$ & $\begin{array}{l}\text { Estimated } \\
\text { Coefficient } \\
\text { (70 mph SL) }\end{array}$ \\
\hline Constant & $11.11(12.37)^{\star \star}$ & $10.88(10.27)^{\star \star}$ & $10.86(8.44)^{\star \star}$ \\
\hline \multicolumn{4}{|l|}{ Driver/household attributes } \\
\hline Male indicator ( 1 if driver is male, 0 otherwise) & - & $0.470(1.37)^{*}$ & - \\
\hline Driver age (years) & - & $-0.088(-2.47)^{\star \star}$ & $-0.129(-2.83)^{\star \star}$ \\
\hline $\begin{array}{l}\text { High income indicator ( } 1 \text { if household's total annual } \\
\text { income is } \$ 75,000 \text { or greater, } 0 \text { otherwise) }\end{array}$ & $1.958(2.06)^{\star *}$ & $2.099(2.71)^{\star *}$ & $1.255(1.52)^{*}$ \\
\hline $\begin{array}{l}\text { Low income indicator ( } 1 \text { if household's total annual } \\
\text { income is less than } \$ 30,000,0 \text { otherwise) }\end{array}$ & $-0.763(-1.17)$ & - & - \\
\hline $\begin{array}{l}\text { Number of children under the age of } 6 \text { years old in the } \\
\text { household }\end{array}$ & $-1.207(-1.54)^{*}$ & $-0.993(-1.48)^{*}$ & $-1.359(-1.87)^{\star *}$ \\
\hline $\begin{array}{l}\text { Late license indicator ( } 1 \text { if driver was first licensed at age } \\
17 \text { or greater, } 0 \text { otherwise) }\end{array}$ & $-3.851(-4.38)^{\star *}$ & $-2.148(-3.00)^{* *}$ & $-1.919(-2.50)^{\star *}$ \\
\hline \multicolumn{4}{|l|}{ Driver opinions } \\
\hline $\begin{array}{l}\text { Good pavement indicator ( } 1 \text { if driver believes pavement } \\
\text { quality on Indiana interstates is good or very good, } 0 \text { o.w.) }\end{array}$ & $1.160(1.46)^{*}$ & $1.067(1.62)^{*}$ & $1.006(1.43)^{*}$ \\
\hline $\begin{array}{l}\text { German prestige indicator ( } 1 \text { if driver believes if German- } \\
\text { brand vehicles are the most prestigious, } 0 \text { otherwise) }\end{array}$ & $1.137(1.85)^{\star *}$ & - & $0.582(1.35)^{\star}$ \\
\hline $\begin{array}{l}\text { Japanese prestige indicator ( } 1 \text { if driver believes if } \\
\text { Japanese-brand vehicles are the most prestigious, } 0 \text { o.w.) }\end{array}$ & $-1.348(-1.25)$ & $-0.814(-1.35)^{*}$ & - \\
\hline $\mathrm{R}^{2}$ & 0.226 & 0.200 & 0.170 \\
\hline Number of observations & & 195 & \\
\hline Equation system $\mathrm{R}^{2}$ & & 0.202 & \\
\hline
\end{tabular}

${ }^{a}$ One-tailed t-test results: ${ }^{* *}$ significantly different from zero at more than $95 \%$ confidence, ${ }^{*}$ significantly diff. from zero at $>90 \%$ conf. 
Individuals that were licensed at age 17 or later had normal driving speeds that were significantly slower under all three speed-limit postings (official licensing age is 16 years in Indiana). The magnitude of this slow down ranged from nearly $4 \mathrm{mph}$ on interstates with $55 \mathrm{mph}$ speed limits to about $2 \mathrm{mph}$ on interstates with $65 \mathrm{mph}$ or $70 \mathrm{mph}$ speed limits. This variable was also found to be significant in some previous safety-related research. For example, using a sample of Washington State drivers, Mannering (1993) found that the number of years after age 16 (the official licensing age in Washington) that women were licensed significantly increased the frequency of their roadway accidents. This variable may be capturing a self-selection process where individuals that are risk adverse or have poorer innate driving skills may wait longer to be licensed and drive more slowly.

Three preference/opinion variables were also found to influence normal driving speed. Those drivers that subjectively rated Indiana interstates as having pavements that were good or very good in quality were more likely to drive faster for all three speed limits considered (about $1 \mathrm{mph}$ faster). This could reflect the impression that high quality pavements are quieter and safer which allows individuals to drive faster while maintaining the same risk/ergonomic comfort levels.

Two variables relating to drivers' impressions of vehicle prestige also played a role in the choice of normal interstate speed under various speed limits. Individuals believing that German manufacturers (Mercedes Benz, BMW and Audi) made the most prestigious cars were more likely to drive faster than the speed limit on interstates posted $55 \mathrm{mph}$ and $70 \mathrm{mph}$. It is likely that this variable is capturing unobserved heterogeneity in the data (that is correlated with vehicle manufacturer perceptions) relating to drivers' perceptions of risk, the utility derived from higher speed driving, and so on. In a similar vein, those drivers believing that Japanese manufacturers made the most prestigious 
vehicles drove slower (less above the speed limit) on interstates posted $55 \mathrm{mph}$ and $65 \mathrm{mph}$.

Given these estimation results, and the observation that drivers' reported speeds are generally well above the speed limit, what does this say about how speed limits should be set? And, what does this say about enforcement of posted speed limits? The results of this study cannot answer these question directly, but it seems clear that drivers do not believe that driving above the speed limit significantly threatens their safety. In fact, a recent survey of 988 Indiana drivers from Bureau of Motor Vehicle offices in Tippecanoe County indicated that $35 \%$ did not believe their safety was threatened until they were driving $20 \mathrm{mph}$ over the speed limit, and an additional 3\% thought that speed was snot a threat to their safety at all (Mannering, 2007). It appears as though setting speed limits that will be respected requires a complex assessment of society's desire for safety and individual drivers' perception of the speed-safety relationship and the trade-offs they are willing to make - which includes the effect of enforcement. 


\section{CHAPTER 7. SUMMARY AND CONCLUSIONS}

The findings of our study are drawn from three statistical models. First we develop a binary model to study the probability that unsafe speed will be determined to be the primary cause of the accident (as reported by the officer). Second, we develop a series of models to study accident severity defined by the injury level of the most severely injured person in the accident. The alternatives are no-injury, injury and fatality. And third, we develop a model to predict normal interstate driving speeds reported by Indiana drivers on interstate highways that are posted $55 \mathrm{mph}, 65 \mathrm{mph}$ and $70 \mathrm{mph}$ under low traffic conditions (level of service A).

For interstate highways, we find that speed limits had no statistically significant effect on the probability that unsafe speed will be listed as the primary cause of the accident except with 2006 data on one vehicle accidents on rural interstates. In this case higher speed limits actually reduced the likelihood that unsafe speed was listed as the primary cause of the accident. As mentioned earlier, some caution should be exercised in interpreting these results because the determination of the officer at the accident scene as to what constitutes the primary cause is an opinion that may be influenced by a multitude of factors (including the posted speed limit itself).

With regard to the effect of speed limits on the severity of vehicle accidents on interstate highways, we find that, for both 2004 and 2006 accident data, speed limits have an insignificant effect on accident severity on interstate highways. This is an important finding because the July 1, 2005 increase in maximum 
interstate speeds from $65 \mathrm{mph}$ to $70 \mathrm{mph}$ has been the focus of considerable media attention. Thus, our analysis covers data from the $70 \mathrm{mph}$ maximum speed limit era (2006) as well as the 65 mph maximum speed limit era (2004). We exclude 2005 data because it is a transition year with speed limits being raised in mid year.

The finding that the speed-safety relationship on interstate highways is not statistically significant, at least in the ranges we consider (maximum of $65 \mathrm{mph}$ in 2004 and $70 \mathrm{mph}$ in 2006), can be the result of a number of factors. First, drivers seem to have a tendency to compensate for posted speed limits. Based on the survey of Indiana drivers conducted in the Fall of 2005, compliance with speed limits improves from $55 \mathrm{mph}$ to $65 \mathrm{mph}$ interstates and $65 \mathrm{mph}$ to $70 \mathrm{mph}$ interstates. Our survey indicated that average driving speeds under free flow conditions were nearly $66 \mathrm{mph}$ on interstates posted $55 \mathrm{mph}, 74 \mathrm{mph}$ on interstates posted $65 \mathrm{mph}$, and almost $78 \mathrm{mph}$ on interstates posted $70 \mathrm{mph}$. Our survey also shows that the higher speed limits of 65 and $70 \mathrm{mph}$ have a lower variance of speeds. The reduction in speed variance as the posted speed limit increases has been discussed in the literature by many including Renski et al., 1999. The "speed variance reduction effect" can be explained by an analysis of Figure 6.1 (Chapter 6 ) - drivers have an optimal speed under specific traffic conditions and tend towards that speed while weighing the probability of being detected by law enforcement.

Countering this reduction in speed variance is the fact that at higher speeds, vehicles travel larger distances during driver reaction times. Thus, drivers have less time to react to changing conditions on the road (such as weather conditions or an object on the roadway surface), resulting in an increase in the frequency and severity of unsafe-speed-related accidents. In addition, a vehicle generally loses stability and roadway traction with an increase in speed because of increased aerodynamic and centripetal forces acting on the vehicle. Also, the 
average kinetic energy of vehicles increases with their speeds. Because this energy must be dissipated during an accident, collisions tend to become more severe as speeds increase.

Another factor that could be playing a role in our interstate speed limit finding is that the high design standards of interstate highways (full access control, high design speeds, median design, shoulders, etc.) may act to mitigate the physics associated with higher speeds. However, with current interstates designed for $70 \mathrm{mph}$ speeds, it is unclear if this finding would hold for maximum speed limits of $75 \mathrm{mph}$ or more. Also, the level of enforcement could be playing a role.

The finding that higher speed limits do not significantly affect the severity of accidents on interstates suggests that the reduction in speed variance, the fact that average speeds do not seem to change that much (average speeds only go up about $3.8 \mathrm{mph}$ between interstates posted $65 \mathrm{mph}$ and $70 \mathrm{mph}$ ) and the high design standards of interstate highways are enough to offset the physics associated with higher speed travel. It also suggests that increasing speed limits on rural interstates from $65 \mathrm{mph}$ to $70 \mathrm{mph}$ (for vehicles under 13 tons gross weight) did not adversely affect the relative severity of accidents on lower and higher speed-limit interstate segments.

For non-interstate highways, the results are quite different. In 2004, for the probability that unsafe speed being listed as the primary cause of the accident, higher speed limits increase this probability for one-vehicle accidents on rural county-maintained roads and for multivehicle accidents on a number of roadway classes. Interestingly, higher speed limits decrease the likelihood of unsafe speed being listed as the primary cause for one-vehicle accidents on rural US routes and urban streets. In 2006, higher speed limits were found to increase this probability for multivehicle accidents on urban state routes but to decrease it for single-vehicle accidents on rural state routes. The conclusion that can be 
drawn from these findings is that the effect of speed limits on the probability of unsafe speed being reported as the primary cause of the accident varies by roadway type and also varies by accident year, suggesting that the increase in speed limits in 2005 has had an effect on the speed-limit causality of accidents and/or officer reporting tendencies.

In terms of accident severities on non-interstate highways, both 2004 and 2006 accident data show that higher speed limits are associated with a greater likelihood of injury and/or fatality on some (but not all) roadway types (county, state, city and US routes) and accident types (single and mutivehicle). The data also show that the speed-limit effects on accident severity vary from 2004 to 2006, suggesting that drivers may still be adjusting their speed behavior in response to the new speed limits and/or this may be reflecting a natural evolution of driver behavior over time.

This study's findings have a number of implications for speed limit polices in the State of Indiana. Our findings suggest that the effect of speed limits on the probability of unsafe speed being listed as the primary cause of the accident and on accident severity are not significant for the speed-limit ranges that we have studied $(55 \mathrm{mph}, 65 \mathrm{mph}, 70 \mathrm{mph})$. Whether this finding would hold true if speed limits were increased further to $75 \mathrm{mph}$ or $80 \mathrm{mph}$ remains an open question. To be sure, the additional speed would increase stopping distances and the energy that would need to be dissipated in the accident. Also, at some point, higher speed limits may start increasing the variance in driver speeds as some drivers continue to drive at or above the speed limit while others drive below the speed limit because the speed limit may have been raised above their "optimum" speed. And, increasing the speed limit above $70 \mathrm{mph}$ would mean increasing it above the roadway's design speed, which could have additional implications. With these factors considered (along with other factors that may come into play such as drivers' variations in driver behavior in response to 
speed limits), we speculate that there will be a point beyond which higher speed limits will significantly increase the severity of accidents on interstates. This is because at some speed-limit level the "traffic calming" effects of higher speed limits will likely become insufficient to offset the physics involved as they now seem to be doing.

With regard to speed limit policies on roadways other than interstate highways, our results suggest that considerable caution should be exercised. The findings show that, on some non-interstate roadway and accident type combinations, higher speed limits significantly increase likelihood that unsafe speed will be listed as the primary cause of the accident and also on some non-interstate roadway and accident type combinations significantly increase the likelihood that accidents will result in injuries and fatalities. Thus, changing speed limits on non-interstate highways should be done on a case-by-case basis taking into account past accident history as well as the specific geometrics and access control of the facility, as these factors can vary widely even within the same class of highways (non-interstate). 


\section{LIST OF REFERENCES}

Abdel-Aty, M., 2003, "Analysis of driver injury severity levels at multiple locations using ordered probit models", Journal of Safety Research, 34, 597

Carson, J., and Mannering, F., 2001, "The effect of ice warning signs on iceaccident frequencies and severities", Accident Analysis and Prevention, 33, 99

Chang, L.-Y., and Mannering, F., 1999, "Analysis of injury severity and vehicle occupancy in truck- and non-truck-involved accidents", Accident Analysis and Prevention, 31, 579

Duncan, C. S., Khattak, A. J., and Council, F. M., 1998, "Applying the ordered probit model to injury severity in truck-passenger car rear-end collisions", Transportation Research Record, 1635, 63

Islam, S., and Mannering, F., 2006, "Driver aging and its effect on male and female single-vehicle accident injuries: some additional evidence", Journal of Safety Research, 37, 267

Karlaftis, M. G., and Tarko, A. P., 1998, "Heterogeneity considerations in accident modeling", Accident Analysis and Prevention, 30, 425

Khan, N. M., 2002, "An analysis of speed limit policies for Indiana", Ph.D. Dissertation, Purdue University, UMI Dissertation Publishing Number: 3104970; http://docs.lib.purdue.edu/dissertations/AAl3104970

Khattak, A. J., 2001, "Injury severity in multivehicle rear-end crashes", Transportation Research Record, 1746, 59

Khorashadi, A., Niemeier, D., Shankar, V., and Mannering, F., 2005, "Differences in rural and urban driver-injury severities in accidents involving large-trucks: an exploratory analysis", Accident Analysis and Prevention, 37, 910

Kockelman, K., Bottom, J., 2006, "Safety impacts and other implications of raised speed limits on high-speed roads", National Cooperative Research Program, Project 17-23, Washington, DC. 
Kockelman, K. M., and Kweon, Y.-J., 2002, "Driver injury severity: an application of ordered probit models", Accident Analysis and Prevention, 34, 313

Kweon, Y.-J., and Kockelman, K. M., 2003, "Overall injury risk to different drivers: combining exposure, frequency, and severity models", Accident Analysis and Prevention, 35, 441

Kweon, Y.-J., and Kockelman, K., 2003, "Driver attitudes and choices: Seatbelt use, speed limits, alcohol consumption, and crash histories", presented at the $82^{\text {nd }}$ Annual Meeting of the Transportation Research Board, Washington, DC.

Lee, J., and Mannering, F., 2002, "Impact of roadside features on the frequency and severity of run-off-roadway accidents: an empirical analysis", Accident Analysis and Prevention, 34, 149

Mannering, F., 1993, "Male/female driver characteristics and accident risk: Some new evidence", Accident Analysis and Prevention, 25(1), 77-84.

Mannering, F., 2007, "Speed limits and safety: A statistical analysis of driver perceptions", Working Paper, Purdue University, West Lafayette, IN.

Mannering, F., Kilareski, W. and Washburn, S. 2005, "Principles of highway engineering and traffic analysis", John Wiley and Sons, New York, NY. Third edition, 384 pages.

Milton, J., and Mannering, F., 1998, "The relationship among highway geometrics, traffic-related elements and motor-vehicle accident frequencies", Transportation, 25, 395

O'Donnell, C. J., and Connor, D. H., 1996, "Predicting the severity of motor vehicle accident injuries using models of ordered multiple choice", Accident Analysis and Prevention, 28, 739

Peltzman, S., 1975, "The effects of automobile safety regulation", Journal of Political Economy, 83, 677-725.

Renski, H., Khattak, A. J., and Council, F. M., 1999, "Effect of speed limit increases on crash injury severity - analysis of single-vehicle crashes on North Carolina interstate highways", Transportation Research Record, 1665, 100

Savolainen, P. T., 2006, "An evaluation of motorcycle safety in Indiana", Ph.D. Dissertation, Purdue University, UMI Dissertation Publishing 
Savolainen, P. T., Mannering, F., 2006a, "Additional evidence on the effectiveness of motorcycle training and motorcyclists' risk-taking behavior", submitted to Transportation Research Record

Savolainen, P. T., Mannering, F., 2006b, "Probabilistic models of motorcyclists' injury severities in single- and multi-vehicle crashes", submitted to Accident Analysis and Prevention

Shankar, V., Mannering, F., Barfield, W., 1996, "Statistical analysis of accident severity on rural freeways", Accident Analysis and Prevention, 28, 391

Shankar, V., Mannering, F., 1998, "Modeling the endogeneity of lane-mean speeds and lane-speed deviations: A structural equations approach", Transportation Research Part A, 32(5), 311-322.

Shankar, V., Milton, J., Mannering, F., 1997, "Modeling accident frequencies as zero-altered probability processes: an empirical inquiry", Accident Analysis and Prevention, 29, 829

Transportation Research Board, 2000, "Highway Capacity Manual”, National Research Council, Washington, DC.

Tsay, R. S., 2002, "Analysis of financial time series: financial econometrics", A Wiley-Interscience Publication, John Wiley \& Sons, Inc

Ulfarsson, G. F., 2001, "Injury severity analysis for car, pickup, sport utility vehicle and minivan drivers: male and female differences", Ph.D. Dissertation, University of Washington, UMI Dissertation Publishing

Ulfarsson, G. F., Mannering, F. L., 2004, "Differences in male and female injury severities in sport-utility vehicle, minivan, pickup and passenger car accidents", Accident Analysis and Prevention, 36, 135

Viscusi, W. K., Carvallo, G., 1994, "The effect of product safety regulation on safety precautions", Risk Analysis, 14, 917-930.

Washington, S. P., Karlaftis, M. G., Mannering, F. L., 2003, "Statistical and econometric methods for transportation data analysis", Chapman \& Hall/CRC, Boca Raton, Florida

Wikipedia, the free encyclopedia; http://en.wikipedia.org

Winston, C., Maheshri, V., Mannering, F., 2006, "An exploration of the offset hypothesis using disaggregate data: The case of airbags and antilock brakes", Journal of Risk and Uncertainty, 32(2), 83-99. 
Yamamoto, T., Shankar, V. N., 2004, "Bivariate ordered-response probit model of driver's and passenger's injury severities in collisions with fixed objects", Accident Analysis and Prevention, 36, 869

Zellner, A., 1962. An efficient method of estimating seemingly unrelated regressions and tests for aggregation bias. Journal of the American Statistical Association 57, 348-368. 
Appendix A.

\section{List of explanatory variables:}

$\mathrm{X}_{3}-$ Collision date

$\mathrm{X}_{4}$ - Day of the week

$X_{5}-$ Collision time

$\mathrm{X}_{13}$ - Construction

(no; yes; buck-up of traffic outside of but due to construction zone)

$\mathrm{X}_{14}$ - Light condition

(daylight; dawn / dusk; dark with street lights on; dark with no lights)

$\mathrm{X}_{15}$ - Weather condition

(clear; cloudy; sleet/hail / freezing rain; fog / smoke / smog; rain; snow; severe cross wind)

$\mathrm{X}_{16}$ - Surface condition

(dry; wet; muddy; snow / slush; ice; loose material on roadway; water)

$\mathrm{X}_{17}$ - Type of median

(drivable; curbed; barrier wall; none)

$\mathrm{X}_{18}$ - Type of roadway junction

(no junction involved; four-way intersection; ramp T-intersection; $Y$-intersection; traffic circle / roundabout; five point or more; interchange)

$\mathrm{X}_{19}$ - Road character

(straight / level; straight / grade; straight / hillcrest; curve / level; curve / grade; curve / hillcrest; non roadway crash)

$\mathrm{X}_{20}$ - Primary contributing circumstance

(alcoholic beverages; illegal drugs; driver asleep or fatigue; prescription drugs; driver illness; unsafe speed; failure to yield right of way; disregard signal / red signal; left of center; improper passing; improper turning; improper lane usage; following too closely; unsafe backing; overcorrecting / oversteering; ran off road right; ran off road left; wrong way on one way; pedestrian action; passenger distraction; violation of license restriction; jackknifing; cell phone usage; other telematics in use; other (explain in narrative); driver distracted [explain in narrative]; speed too fast for weather conditions; engine failure or defective; accelerator failure or defective; brake failure or defective; tire failure or defective; 
headlight defective or not on; other lights defective; steering failure; window / windshield defective; oversize / overweight load; insecure / leaky load; tow hitch failure; other explained in narrative; glare; roadway surface condition; holes / ruts in surface; shoulder defective; road under construction; severe crosswinds; obstruction not marked; lane marking obscured; view obstructed; animal on roadway; traffic control problem; other [explained in narrative]; utility work)

$X_{22}$ - Time when help arrived

$\mathrm{X}_{25}$ - Vehicle type, considered for the vehicle at fault, i.e. for the vehicle that contributed to the primary cause of an accident

(passenger car / station wagon; pickup; van; sport utility vehicle; truck [single 2 axle, 6 tires]; truck [single 3 or more axles]; truck / trailer [not semi]; tractor / one semi trailer; tractor / double trailer; tractor / triple trailer tractor [cab only, no trailer]; motor home / recreational vehicle; motorcycle; bus/seats 9-15 persons with driver; bus / seats 15+ persons with driver; school bus; unknown type; farm vehicle; combination vehicle; pedestrian; bicycle)

$\mathrm{X}_{26}$ - Vehicle year, considered for all vehicles involved

$X_{27}-$ Number of occupants, considered for all vehicles involved

$\mathrm{X}_{28}$ - Vehicle license state, considered for the vehicle at fault, i.e. for the vehicle that contributed to the primary cause of an accident (Indiana; Indiana's neighboring states [IL, $\mathrm{KY}, \mathrm{OH}, \mathrm{MI}]$; other US states; Canada / Mexico / U.S. Territories; other foreign countries)

$\mathrm{X}_{29}$ - Speed limit, considered only if known and the same speed limit value for all vehicles involved

$X_{30}$ - Road type, considered for the vehicle at fault, i.e. for the vehicle contributed to the primary cause of an accident (one lane [one way]; two lanes [one way]; multi-lanes [one way]; two lanes [two way]; multi-lane undivided [two way]; multi-lane undivided 2way left [two way]; multi-lane divided 3 or more lanes [two way]; alley; private drive)

$X_{31}$ - Traffic control, considered for the vehicle at fault, i.e. for the vehicle contributed to the primary cause of an accident

(officer / crossing guard / flagman; RR crossing gate / flagman; $R R$ crossing flashing signal; $R R$ crossing sign; traffic control signal; flashing signal; stop sign; yield sign; lane control; no passing zone; other regulatory sign / marking; none) 
$\mathrm{X}_{33}$ - Fire, considered for all vehicles involved (no; yes)

$\mathrm{X}_{34}$ - Driver age, considered for all drivers involved

$\mathrm{X}_{35}$ - Driver gender, considered for all drivers involved 
Appendix B.

Table B.1 Road classes \& accident types in 2004 accident causation study

\begin{tabular}{|c|c|c|c|c|c|c|c|}
\hline \multirow[b]{3}{*}{ \# } & \multirow{3}{*}{\multicolumn{3}{|c|}{$\begin{array}{c}\text { Road-class-accident-type } \\
\text { combination }\end{array}$}} & \multicolumn{4}{|c|}{ Number of observations } \\
\hline & & & & \multirow[b]{2}{*}{ all } & \multicolumn{3}{|c|}{ available for the models ${ }^{*}$} \\
\hline & & & & & total & unsafe- & other \\
\hline 1 & \multirow{6}{*}{ 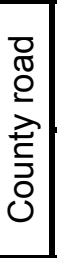 } & \multirow{3}{*}{$\stackrel{\bar{\pi}}{\stackrel{0}{2}}$} & $\left(\operatorname{car} / S U V^{* *}\right)+(\operatorname{car} / S U V)$ & 7249 & 5198 & 518 & 4680 \\
\hline 2 & & & $\left(\right.$ car/SUV)+(truck $\left.{ }^{* * *}\right)$ & 647 & 617 & 28 & 589 \\
\hline 3 & & & one vehicle & 18045 & 11998 & 1877 & 10121 \\
\hline 4 & & \multirow{3}{*}{ 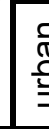 } & (car/SUV)+(car/SUV) & 1854 & 1490 & 97 & 1393 \\
\hline 5 & & & (car/SUV)+(truck) & 143 & 121 & 7 & 114 \\
\hline 6 & & & one vehicle & 972 & 689 & 142 & 547 \\
\hline 7 & \multirow{6}{*}{ 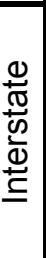 } & \multirow{3}{*}{ 宽 } & (car/SUV)+(car/SUV) & 1041 & 995 & 168 & 827 \\
\hline 8 & & & (car/SUV)+(truck) & 811 & 338 & 77 & 311 \\
\hline 9 & & & one vehicle & 3347 & 1617 & 430 & 1187 \\
\hline 10 & & \multirow{3}{*}{\begin{tabular}{|l}
$\frac{7}{\pi}$ \\
o \\
$\frac{0}{J}$
\end{tabular}} & (car/SUV)+(car/SUV) & 2227 & 1386 & 131 & 1255 \\
\hline 11 & & & (car/SUV)+(truck) & 3306 & 922 & 84 & 838 \\
\hline 12 & & & one vehicle & 1605 & 1442 & 463 & 979 \\
\hline 13 & \multirow{6}{*}{ 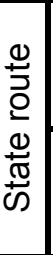 } & \multirow{3}{*}{ 㞼 } & (car/SUV)+(car/SUV) & 4774 & 2311 & 170 & 2141 \\
\hline 14 & & & (car/SUV)+(truck) & 682 & 665 & 41 & 624 \\
\hline 15 & & & one vehicle & 9775 & 6432 & 540 & 5892 \\
\hline 16 & & \multirow{3}{*}{$\begin{array}{l}\frac{}{\mathbb{d}} \\
\text { 일 }\end{array}$} & $(\mathrm{car} / \mathrm{SUV})+(\mathrm{car} / \mathrm{SUV})$ & 7999 & 4698 & 191 & 4507 \\
\hline 17 & & & (car/SUV)+(truck) & 636 & 633 & 16 & 617 \\
\hline 18 & & & one vehicle & 1488 & 1004 & 99 & 905 \\
\hline 19 & \multirow{6}{*}{ 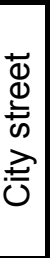 } & \multirow{3}{*}{ 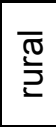 } & $(\mathrm{car} / \mathrm{SUV})+(\mathrm{car} / \mathrm{SUV})$ & 3778 & 2648 & 155 & 2493 \\
\hline 20 & & & (car/SUV)+(truck) & 261 & 261 & 9 & 252 \\
\hline 21 & & & one vehicle & 2387 & 2187 & 265 & 1922 \\
\hline 22 & & \multirow{3}{*}{ 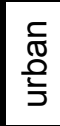 } & (car/SUV)+(car/SUV) & 62701 & 50180 & 1901 & 48279 \\
\hline 23 & & & $($ car/SUV)+(truck) & 3574 & 3105 & 85 & 3200 \\
\hline 24 & & & one vehicle & 12205 & 7988 & 1134 & 6854 \\
\hline 25 & \multirow{6}{*}{ 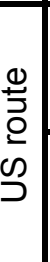 } & \multirow{3}{*}{$\overline{\frac{\pi}{2}}$} & (car/SUV)+(car/SUV) & 2588 & 2005 & 152 & 1853 \\
\hline 26 & & & (car/SUV)+(truck) & 566 & 563 & 52 & 511 \\
\hline 27 & & & one vehicle & 4202 & 2667 & 247 & 2420 \\
\hline 28 & & \multirow{3}{*}{$\begin{array}{l}\frac{1}{\mathbb{\pi}} \\
\frac{0}{5} \\
\frac{0}{J}\end{array}$} & (car/SUV)+(car/SUV) & 6895 & 6462 & 328 & 6134 \\
\hline 29 & & & (car/SUV)+(truck) & 750 & 734 & 37 & 697 \\
\hline 30 & & & one vehicle & 1061 & 988 & 97 & 891 \\
\hline
\end{tabular}

* - observations available for the best estimated statistical models after exclusion of all missing observations

** - "SUV" includes sport utility vehicles, pickups and vans

*** - "truck" includes any possible kind of truck or tractor 
Table B.2 Road classes \& accident types in 2006 accident causation study

\begin{tabular}{|c|c|c|c|c|c|c|c|}
\hline \multirow[b]{3}{*}{ \# } & \multirow{3}{*}{\multicolumn{3}{|c|}{$\begin{array}{c}\text { Road-class-accident-type } \\
\text { combination }\end{array}$}} & \multicolumn{4}{|c|}{ Number of observations } \\
\hline & & & & \multirow[b]{2}{*}{ all } & \multicolumn{3}{|c|}{ available for the models ${ }^{*}$} \\
\hline & & & & & tatol & unsafe- & other \\
\hline 1 & \multirow{7}{*}{ 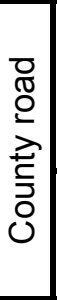 } & \multirow{4}{*}{$\stackrel{\overline{0}}{\frac{\pi}{2}}$} & $\left(\mathrm{car} / \mathrm{SUV}^{* *}\right)+(\mathrm{car} / \mathrm{SUV})$ & 5956 & 1698 & 111 & 1587 \\
\hline $2 a$ & & & $($ car $)+\left(\right.$ truck $\left.^{* \star *}\right)$ & 194 & 194 & 6 & 188 \\
\hline $2 b$ & & & $(\mathrm{SUV})+\left(\right.$ truck $\left.^{* * *}\right)$ & 150 & 126 & 2 & 124 \\
\hline 3 & & & one vehicle & 16132 & 3518 & 500 & 3018 \\
\hline 4 & & \multirow{3}{*}{$\begin{array}{l}0 \\
\frac{1}{2}\end{array}$} & (car/SUV)+(car/SUV) & 1485 & 1483 & 66 & 1417 \\
\hline 5 & & & (car/SUV)+(truck) & 83 & 79 & 5 & 74 \\
\hline 6 & & & one vehicle & 797 & 752 & 103 & 649 \\
\hline $7 a$ & \multirow{9}{*}{ 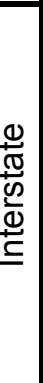 } & \multirow{5}{*}{ 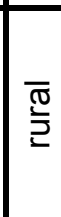 } & (car)+(car) & 395 & 354 & 37 & 317 \\
\hline $7 \mathrm{~b}$ & & & (car)+(SUV) & 518 & 476 & 59 & 417 \\
\hline $7 c$ & & & (SUV)+(SUV) & 210 & 209 & 26 & 183 \\
\hline 8 & & & (car/SUV)+(truck) & 757 & 742 & 97 & 645 \\
\hline 9 & & & one vehicle & 3730 & 3637 & 489 & 3148 \\
\hline 10 & & \multirow{4}{*}{$\begin{array}{l}\frac{5}{\pi} \\
\frac{0}{\zeta} \\
\frac{0}{\zeta}\end{array}$} & (car/SUV)+(car/SUV) & 2392 & 2203 & 172 & 2031 \\
\hline $11 \mathrm{a}$ & & & (car)+(truck) & 627 & 541 & 47 & 494 \\
\hline $11 \mathrm{~b}$ & & & (SUV)+(truck) & 220 & 209 & 20 & 189 \\
\hline 12 & & & one vehicle & 1883 & 303 & 75 & 228 \\
\hline 13 & \multirow{6}{*}{ 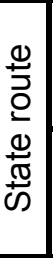 } & \multirow{3}{*}{ 㞼 } & (car/SUV)+(car/SUV) & 4577 & 4377 & 217 & 4160 \\
\hline 14 & & & (car/SUV)+(truck) & 522 & 511 & 30 & 481 \\
\hline 15 & & & one vehicle & 10155 & 9421 & 546 & 8875 \\
\hline 16 & & \multirow{3}{*}{ 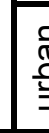 } & (car/SUV)+(car/SUV) & 7461 & 6241 & 220 & 6021 \\
\hline 17 & & & (car/SUV)+(truck) & 509 & 508 & 23 & 485 \\
\hline 18 & & & one vehicle & 1700 & 1603 & 126 & 1477 \\
\hline 19 & \multirow{8}{*}{ 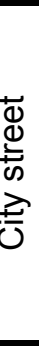 } & \multirow{4}{*}{$\frac{\overline{\sqrt[0]{0}}}{\underline{2}}$} & (car/SUV)+(car/SUV) & 3778 & 1294 & 55 & 1239 \\
\hline $20 a$ & & & (car)+(truck) & 97 & 97 & 4 & 93 \\
\hline $20 b$ & & & (SUV)+(truck) & 57 & 57 & 3 & 54 \\
\hline 21 & & & one vehicle & 2104 & 1929 & 225 & 1704 \\
\hline 22 & & \multirow{3}{*}{ 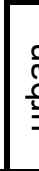 } & (car/SUV)+(car/SUV) & 50034 & 20704 & 563 & 20141 \\
\hline $23 a$ & & & (car)+(truck) & 1526 & 945 & 25 & 920 \\
\hline $23 b$ & & & (SUV)+(truck) & 788 & 481 & 8 & 473 \\
\hline 24 & & & one vehicle & 110682 & 9011 & 945 & 8066 \\
\hline 25 & \multirow{7}{*}{$\begin{array}{l}\stackrel{\Phi}{J} \\
\stackrel{0}{0} \\
\stackrel{\infty}{\supset}\end{array}$} & \multirow{3}{*}{ 苞 } & (car/SUV)+(car/SUV) & 2478 & 2353 & 129 & 2224 \\
\hline 26 & & & (car/SUV)+(truck) & 474 & 457 & 27 & 430 \\
\hline 27 & & & one vehicle & 4204 & 4151 & 262 & 3889 \\
\hline 28 & & \multirow{4}{*}{$\begin{array}{l}\frac{c}{\widetilde{N}} \\
\stackrel{0}{J}\end{array}$} & (car/SUV)+(car/SUV) & 6925 & 6140 & 206 & 5934 \\
\hline $29 a$ & & & (car)+(truck) & 346 & 342 & 12 & 330 \\
\hline $29 \mathrm{~b}$ & & & (SUV)+(truck) & 226 & 226 & 2 & 224 \\
\hline 30 & & & one vehicle & 1209 & 1148 & 90 & 1058 \\
\hline
\end{tabular}

* - observations available for the best estimated statistical models after exclusion of all missing observations

** - "SUV" includes sport utility vehicles, pickups and vans

*** - "truck" includes any possible kind of truck or tractor 
Table B.3 Binary logit models for 2004 accident causation ${ }^{19}$

\begin{tabular}{|c|c|c|c|c|c|c|c|c|}
\hline \multirow[t]{2}{*}{ \# } & & \multirow{2}{*}{\multicolumn{2}{|c|}{ Model name }} & \multicolumn{2}{|c|}{ Log-likelihood } & \multirow{2}{*}{$R^{2}$} & \multicolumn{2}{|c|}{ Coefficient (t-ratio) } \\
\hline & & & & model & restricted $^{*}$ & & $X_{29}$ & constant \\
\hline 1 & \multirow{3}{*}{\multicolumn{2}{|c|}{ 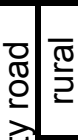 }} & (car/SUV)+(car/SUV) & -1426.7 & -1685.9 & .154 & & $-1.14(-6.71)$ \\
\hline 2 & & & (car/SUV)+(truck) & -87.04 & -113.95 & .236 & & \\
\hline 3 & & & one vehicle & -4468.1 & -5203.8 & .141 & $.00859(2.83)$ & $-.743(-3.30)$ \\
\hline 4 & \multirow{3}{*}{\multicolumn{2}{|c|}{ S. }} & (car/SUV)+(car/SUV) & -246.94 & -307.15 & .196 & $.0368(2.24)$ & $-1.42(-2.12)$ \\
\hline $5^{\text {** }}$ & & & (car/SUV)+(truck) & -16.14 & -26.74 & .396 & & $-2.57(-2.00)$ \\
\hline 6 & & \begin{tabular}{l}
$\frac{c}{\mathbb{0}}$ \\
$\frac{0}{5}$ \\
\multirow{2}{*}{}
\end{tabular} & one vehicle & -305.79 & -350.52 & .128 & & \\
\hline 7 & \multirow{3}{*}{\multicolumn{2}{|c|}{ 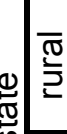 }} & (car/SUV)+(car/SUV) & -327.44 & -451.78 & .275 & & $.985(-2.40)$ \\
\hline 8 & & & (car/SUV)+(truck) & -128.79 & -193.32 & .334 & & \\
\hline 9 & & & one vehicle & -564.03 & -936.51 & .398 & & \\
\hline 10 & $\stackrel{\infty}{\Phi}$ & \multirow{3}{*}{$\begin{array}{l}\frac{c}{\mathbb{N}} \\
\frac{2}{3}\end{array}$} & (car/SUV)+(car/SUV) & -339.99 & -436.39 & .221 & & $-1.52(-4.83)$ \\
\hline 11 & 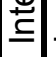 & & (car/SUV)+(truck) & -203.66 & -306.50 & .336 & & $-.928(-2.92)$ \\
\hline 12 & & & one vehicle & -700.30 & -942.58 & .257 & & \\
\hline 13 & \multirow{3}{*}{\multicolumn{2}{|c|}{$\frac{0}{5}$}} & (car/SUV)+(car/SUV) & -537.67 & -607.23 & .115 & & $-1.72(-6.68)$ \\
\hline 14 & & & (car/SUV)+(truck) & -128.69 & -153.94 & .164 & & $-2.08(-6.50)$ \\
\hline 15 & & & one vehicle & -1452.8 & -1854.5 & .217 & & \\
\hline 16 & \multirow{3}{*}{\multicolumn{2}{|c|}{ 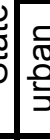 }} & $($ car/SUV)+(car/SUV) & -716.67 & -798.76 & .103 & $.0212(2.51)$ & $-2.77(-7.83)$ \\
\hline 17 & & & (car/SUV)+(truck) & -57.96 & -74.64 & .224 & & $-4.05(-8.74)$ \\
\hline 18 & & & one vehicle & -274.39 & -323.30 & .103 & & $-1.03(-2.08)$ \\
\hline 19 & & \multirow{3}{*}{ 을 } & $($ (car/SUV)+(car/SUV) & -525.56 & -586.00 & .094 & $.0225(2.50)$ & $-2.87(-5.48)$ \\
\hline 20 & + & & (car/SUV)+(truck) & -35.71 & -39.15 & .088 & & $-3.69(-8.93)$ \\
\hline 21 & $\stackrel{\oplus}{=}$ & & one vehicle & -681.68 & -807.55 & .156 & & \\
\hline 22 & $\geqslant$ & \multirow{3}{*}{$\begin{array}{l}\frac{1}{\pi} \\
\frac{0}{J} \\
\frac{0}{J}\end{array}$} & (car/SUV)+(car/SUV) & -6259.1 & -7117.7 & .121 & & $-2.43(-20.9)$ \\
\hline 23 & $\bar{U}$ & & (car/SUV)+(truck) & -323.90 & -389.67 & .169 & & $-.983(-2.22)$ \\
\hline 24 & & & one vehicle & -2776.2 & -3263.2 & .149 & $-.0117(-3.82)$ & \\
\hline 25 & \multirow{3}{*}{\multicolumn{2}{|c|}{ 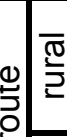 }} & $($ car/SUV)+(car/SUV) & -404.95 & -475.98 & .149 & & $-2.14(-8.87)$ \\
\hline 26 & & & (car/SUV)+(truck) & -147.16 & -173.39 & .151 & & $-.822(-3.51)$ \\
\hline 27 & & & one vehicle & -593.93 & -822.88 & .278 & $-.0422(-3.17)$ & $3.48(4.66)$ \\
\hline 28 & \multirow{3}{*}{$\stackrel{\omega}{\supset}$} & \multirow{3}{*}{\begin{tabular}{l|}
$\frac{c}{\widetilde{J}}$ \\
$\frac{0}{5}$ \\
\end{tabular}} & (car/SUV)+(car/SUV) & -1088.6 & -1200.4 & .093 & $.0181(2.33)$ & $-2.32(-6.06)$ \\
\hline 29 & & & (car/SUV)+(truck) & -117.72 & -146.59 & .197 & & \\
\hline 30 & & & one vehicle & -206.07 & -255.30 & .193 & & $-.916(-2.15)$ \\
\hline
\end{tabular}

- positive coefficient

- negative coefficient

* - restricted log-likelihood found by setting all coefficients except intercepts to zero

** - models are estimated by using procedure A on page 32, except the models marked by bold numbers and estimated by using procedure $B$ on page 33

" $\mathrm{X}_{29}$ " - "posted speed limit (if the same for all vehicles involved)" quantitative variable

"constant" - "constant term (intercept)" quantitative variable

19 Refer to Equation (2.3), where outcomes "1" and "2" correspond to "unsafe-speed-related cause" and "any other cause". Only statistically significant coefficients, which are components of vector $\boldsymbol{\beta}_{1}$ in Equation (2.3), are given in the table. 
Table B.3 (Continued)

\begin{tabular}{|c|c|c|c|c|c|c|}
\hline \multirow{2}{*}{$\#$} & \multicolumn{5}{|c|}{ Coefficient (t-ratio) } \\
\hline & wint $\left[\mathrm{X}_{3}\right]$ & mon $\left[\mathrm{X}_{4}\right]$ & tues $\left[\mathrm{X}_{4}\right]$ & wed $\left[\mathrm{X}_{4}\right]$ & fr $\left[\mathrm{X}_{4}\right]$ & sat $\left[\mathrm{X}_{4}\right]$ \\
\hline 1 & & $.382(2.91)$ & & & & \\
\hline 2 & & & & & & \\
\hline 3 & $.370(6.32)$ & & & & & \\
\hline 4 & & & & & & \\
\hline 5 & & & & & & \\
\hline 6 & & & & & & \\
\hline 7 & $.916(4.40)$ & & & & & \\
\hline $\mathbf{8}$ & & & & & & \\
\hline 9 & $1.34(8.52)$ & & & & & \\
\hline 10 & & & & & & \\
\hline 11 & $.630(4.44)$ & & & & & \\
\hline 12 & $.662(4.59)$ & & & & & \\
\hline 13 & $.613(3.57)$ & & & & & \\
\hline 14 & & & & & & \\
\hline 15 & $.562(5.60)$ & & & & & \\
\hline 16 & & & & & & \\
\hline 17 & $1.59(2.39)$ & & & & & \\
\hline 18 & & & & & & \\
\hline 19 & & & & & & \\
\hline 20 & & & & & & \\
\hline 21 & $.426(2.94)$ & & & $-.608(-2.23)$ & & \\
\hline 22 & $.425(7.71)$ & & & & & \\
\hline 23 & & & & & & \\
\hline 24 & $.262(3.57)$ & & & & & \\
\hline 25 & & & & & & \\
\hline 26 & & & & & & \\
\hline 27 & $.514(3.13)$ & & & & & \\
\hline 28 & $.431(3.52)$ & & & & & \\
\hline 29 & & & & & & \\
\hline 30 & & & & & & \\
\hline
\end{tabular}

- positive coefficient

- negative coefficient

"wint" - "winter season" indicator variable

"mon" - "Monday" indicator variable

"tues" - "Tuesday" indicator variable

"wed" - "Wednesday" indicator variable

"fr" _ _Friday" indicator variable

"sat" _ - "Saturday" indicator variable 
Table B.3 (Continued)

\begin{tabular}{|c|c|c|c|c|c|c|c|}
\hline \multirow{2}{*}{ \# } & \multicolumn{7}{|c|}{ Coefficient (t-ratio) } \\
\hline & sund $\left[\mathrm{X}_{4}\right]$ & wday $\left[\mathrm{X}_{4}\right]$ & $\begin{array}{c}\text { jobend } \\
{\left[\mathrm{X}_{5}\right]}\end{array}$ & peak $\left[X_{5}\right]$ & nigh $\left[X_{5}\right]$ & $\begin{array}{c}\text { nocons } \\
{\left[\mathrm{X}_{13}\right]}\end{array}$ & light $\left[X_{14}\right]$ \\
\hline \multicolumn{8}{|l|}{1} \\
\hline \multicolumn{8}{|l|}{2} \\
\hline 3 & & & & & & & $.416(7.64)$ \\
\hline 4 & & & & & & & $-.515(-2.28)$ \\
\hline \multicolumn{8}{|l|}{5} \\
\hline 6 & & & & $.535(2.27)$ & & & \\
\hline 7 & & & & & & & $-.509(-2.35)$ \\
\hline 8 & $1.14(2.29)$ & & & & & & \\
\hline \multicolumn{8}{|l|}{9} \\
\hline 10 & & $-.660(-2.90)$ & & & & & \\
\hline \multicolumn{8}{|l|}{11} \\
\hline 12 & & $-.333(-2.51)$ & & & & $962(4.98)$ & \\
\hline \multicolumn{8}{|l|}{13} \\
\hline \multicolumn{8}{|l|}{14} \\
\hline 15 & & & & & & & $.693(7.26)$ \\
\hline \multicolumn{8}{|c|}{$1.64(3.67)$} \\
\hline \multicolumn{8}{|l|}{17} \\
\hline 18 & & & & & & & $.745(2.50)$ \\
\hline 19 & $.597(2.39)$ & & $382(2.07)$ & & & & \\
\hline \multicolumn{8}{|l|}{20} \\
\hline \multirow{2}{*}{\multicolumn{8}{|c|}{$\frac{21}{21}$}} \\
\hline 22 & & & & & & & \\
\hline \multicolumn{8}{|c|}{$.677(-2.25)$} \\
\hline \multicolumn{8}{|l|}{24} \\
\hline \multicolumn{8}{|l|}{25} \\
\hline \multirow{2}{*}{\multicolumn{8}{|c|}{26}} \\
\hline & & & & & & & \\
\hline 28 & & $-.280(-2.03)$ & & & & & \\
\hline 29 & & $-1.08(-3.42)$ & & & & & \\
\hline 30 & & & & & & & \\
\hline
\end{tabular}

- positive coefficient

- negative coefficient

"sund" - "Sunday" indicator variable

"wday" - "weekday (Monday through Friday)" indicator variable

"jobend" - "evening rush hours: from 16:00 to 19:00" indicator variable ${ }^{20}$

"peak" - "rush hours: 7:00 to 9:00 OR 17:00 to 19:00" indicator variable

"nigh" - "late night hours: 1:00 to 5:00" indicator variable

"nocons" - "no construction at the accident location" indicator variable

"light" - - "daylight time OR street lights lit up during dark time" indicator variable

${ }^{20}$ We use military 24-hour time everywhere in our research. 
Table B.3 (Continued)

\begin{tabular}{|c|c|c|c|c|c|c|}
\hline \multirow{2}{*}{ \# } & \multicolumn{6}{|c|}{ Coefficient (t-ratio) } \\
\hline & dark $\left[\mathrm{X}_{14}\right]$ & day $\left[X_{14}\right]$ & precip $\left[\mathrm{X}_{15}\right]$ & snow $\left[\mathrm{X}_{15}\right]$ & $\operatorname{dry}\left[\mathrm{X}_{16}\right]$ & slush $\left[\mathrm{X}_{16}\right]$ \\
\hline 1 & & & & & $-1.90(-17.7)$ & \\
\hline 2 & & & & & $-2.32(-5.00)$ & \\
\hline 3 & & & & & $-1.20(-20.0)$ & \\
\hline 4 & & & & & $-1.91(-7.22)$ & \\
\hline 5 & & & & & $-4.40(-2.44)$ & \\
\hline 6 & & & & & $\mid-1.25(-6.25)$ & \\
\hline 7 & & & & & $-2.08(-9.83)$ & \\
\hline 8 & & & & & $-2.57(-7.55)$ & \\
\hline 9 & $-.627(-4.10)$ & & $.466(3.04)$ & & $-2.88(-11.5)$ & \\
\hline 10 & & & & $1.66(5.37)$ & $-1.72(-7.58)$ & \\
\hline 11 & & $-.599(-2.28)$ & & & $-2.46(-8.85)$ & \\
\hline 12 & & & $.947(5.24)$ & & $-1.38(-7.94)$ & \\
\hline 13 & & & & & \begin{tabular}{|l|}
$-1.21(-7.05)$ \\
\end{tabular} & \\
\hline 14 & & & & $1.50(2.88)$ & $-1.39(-3.66)$ & \\
\hline 15 & & & $.317(2.66)$ & & $-1.55(-12.0)$ & \\
\hline 16 & & & & & $-1.61(-10.5)$ & \\
\hline 17 & & & & & & $1.49(2.29)$ \\
\hline 18 & & & $.670(.318)$ & & $-.941(-2.83)$ & \\
\hline 19 & & & & & $-1.28(-7.36)$ & \\
\hline 20 & & $.562(4.11)$ & $.376(2.22)$ & & $-1.01(-6.33)$ & $2.30(3.00)$ \\
\hline 21 & & & & & & \\
\hline 22 & & & $.468(6.69)$ & & $-1.30(-17.5)$ & \\
\hline 23 & & & & & $-1.70(-7.20)$ & \\
\hline 24 & & & $.241(2.68)$ & & $-1.01(-11.5)$ & \\
\hline 25 & & & & & $-1.74(-8.98)$ & \\
\hline 26 & $-1.58(-2.54)$ & & & & $-1.90(-6.04)$ & \\
\hline 27 & $-1.01(-6.32)$ & & & & $\mid-2.06(-10.7)$ & \\
\hline 28 & & & & & $-1.31(-11.1)$ & \\
\hline 29 & & $.752(-2.06)$ & & & $\mid-1.21(-3.35)$ & \\
\hline 30 & & & & & $-1.93(-6.62)$ & \\
\hline
\end{tabular}

- positive coefficient

- negative coefficient

"dark" - "dark time with no street lights" indicator variable

"day" - - "daylight time" indicator variable

"precip" - "precipitation: rain OR snow OR sleet OR hail OR freezing rain" indicator variable

"snow" - "snowing weather" indicator variable

"dry" _ - "roadway surface is dry" indicator variable

"slush" - "roadway surface is covered by snow/slush" indicator variable 
Table B.3 (Continued)

\begin{tabular}{|c|c|c|c|c|c|c|}
\hline \multirow{2}{*}{ \# } & \multicolumn{6}{|c|}{ Coefficient (t-ratio) } \\
\hline & $\operatorname{driv}\left[X_{17}\right]$ & wall $\left[\mathrm{X}_{17}\right]$ & nojun $\left[\mathrm{X}_{18}\right]$ & $\operatorname{ramp}\left[\mathrm{X}_{18}\right]$ & way4 $\left[\mathrm{X}_{18}\right]$ & $\mathrm{T}\left[\mathrm{X}_{18}\right]$ \\
\hline 1 & & & $482(4.03)$ & & & \\
\hline 2 & & & & & & \\
\hline 3 & & & $-.300(-3.83)$ & & & \\
\hline 4 & & & & & & \\
\hline 5 & & & & & & \\
\hline 6 & & & & & & \\
\hline 7 & & & $.976(3.15)$ & & & \\
\hline 8 & & & & $-1.90(-2.27)$ & & \\
\hline 9 & & & & & & \\
\hline 10 & & & & & & \\
\hline 11 & & & & & & \\
\hline 12 & $-.698(-3.66)$ & & $.533(-3.03)$ & & & \\
\hline 13 & & & $.465(2.62)$ & & & \\
\hline 14 & & & & & & $905(2.19)$ \\
\hline 15 & & &. $.540(-4.09)$ & & & \\
\hline 16 & & & & & $-.462(-2.76)$ & \\
\hline 17 & & & & & & \\
\hline 18 & & & $-.560(-2.31)$ & & & \\
\hline 19 & $-.542(-2.99)$ & & & & & \\
\hline 20 & & & & & & \\
\hline 21 & & & & & & \\
\hline 22 & & & & & $-.210(-3.76)$ & \\
\hline 23 & & $1.47(3.75)$ & & & & \\
\hline 24 & & & & $1.22(5.13)$ & & \\
\hline 25 & & & .691(3.49) & & & \\
\hline 26 & & & & & & \\
\hline 27 & & $1.31(5.24)$ & & & & \\
\hline 28 & & & .383(3.17) & & & \\
\hline 29 & & & & & $-1.30(-2.85)$ & \\
\hline 30 & & & & & & \\
\hline
\end{tabular}

- positive coefficient

- negative coefficient

"driv" - "road median is a drivable" indicator variable

"wall" _ "road median is a barrier wall" indicator variable

"nojun" - "no road junction at the accident location" indicator variable

"ramp" - "accident location is near or on a ramp" indicator variable

"way4" - "accident location is at a 4-way intersection" indicator variable

"T" - "accident location is at a T-intersection" indicator variable 
Table B.3 (Continued)

\begin{tabular}{|c|c|c|c|c|c|c|}
\hline \multirow{2}{*}{ \# } & \multicolumn{6}{|c|}{ Coefficient (t-ratio) } \\
\hline & curve $\left[\mathrm{X}_{19}\right]$ & $\operatorname{sg}\left[X_{19}\right]$ & sl $\left[X_{19}\right]$ & $\operatorname{str}\left[X_{19}\right]$ & $\operatorname{cl}\left[\mathrm{X}_{19}\right]$ & $\mathrm{h} \mid 5\left[\mathrm{X}_{22}\right]$ \\
\hline 1 & & & & & & \\
\hline 2 & & & & & & \\
\hline 3 & $.892(14.9)$ & & & & & \\
\hline 4 & & & $-1.08(-4.10)$ & & & \\
\hline 5 & & & & & & \\
\hline 6 & & & & & $.892(3.42)$ & \\
\hline 7 & & & & & & .499(2.13) \\
\hline 8 & & & & & & \\
\hline 9 & & & $-.482(-3.26)$ & & & $.421(2.24)$ \\
\hline 10 & & & & & & \\
\hline 11 & & & & & & \\
\hline 12 & & & & $-.983(-5.73)$ & & \\
\hline 13 & & & & & & \\
\hline 14 & & & & & & \\
\hline 15 & & & & $-.959(-9.56)$ & & \\
\hline 16 & & & & & & \\
\hline 17 & & & & & & $-1.92(-2.49)$ \\
\hline 18 & $.582(2.18)$ & & & & & \\
\hline 19 & $.865(3.47)$ & & & & & \\
\hline 20 & & & $-1.14(-8.21)$ & & & \\
\hline 21 & & & & & & \\
\hline 22 & $.884(9.43)$ & & & & & \\
\hline 23 & & & & & & \\
\hline 24 & (896(11.5) & & & & & \\
\hline 25 & & & & & & $-.463(-2.05)$ \\
\hline 26 & & & & & & $-.856(-2.00)$ \\
\hline 27 & & & & $-1.35(-7.54)$ & & \\
\hline 28 & & $.608(3.18)$ & & & & $-.283(-2.32)$ \\
\hline 29 & & & & & & \\
\hline 30 & .981(3.22) & & & & & \\
\hline
\end{tabular}

- positive coefficient

- negative coefficient

"curve" - "road is at curve" indicator variable

"sg" - - "road is straight AND at grade" indicator variable

"sl" _ - "road is straight AND level" indicator variable

"str" _ _road is straight" indicator variable

"cl" _ - "road is at-curve AND level" indicator variable

"hl5" _ - "help arrived in 5 minutes or less after the crash" indicator variable 
Table B.3 (Continued)

\begin{tabular}{|c|c|c|c|c|c|c|}
\hline \multirow{2}{*}{ \# } & \multicolumn{6}{|c|}{ Coefficient (t-ratio) } \\
\hline & $\mathrm{hl} 10\left[\mathrm{X}_{22}\right]$ & hg30 $\left[X_{22}\right]$ & $\mathrm{hg} 60\left[\mathrm{X}_{22}\right]$ & $\operatorname{car}\left[\mathrm{X}_{25}\right]$ & heavy $\left[X_{25}\right]$ & moto $\left[X_{25}\right]$ \\
\hline 1 & & & & & & \\
\hline 2 & & & & & & \\
\hline 3 & . $.141(2.41)$ & & & $.220(3.87)$ & & \\
\hline 4 & & & & & & \\
\hline 5 & & & & & & \\
\hline 6 & & & & & & \\
\hline 7 & & & & & & \\
\hline 8 & & & & & $-2.32(-5.44)$ & \\
\hline 9 & & & & & & \\
\hline 10 & & $-.736(-2.16)$ & & & & \\
\hline 11 & & & & & $-1.86(-5.15)$ & \\
\hline 12 & & $-.393(-2.12)$ & & & & \\
\hline 13 & & & & & & \\
\hline 14 & & & & & & \\
\hline 15 & & & & & & \\
\hline 16 & & & & & & \\
\hline 17 & & & & & & \\
\hline 18 & & & & & & \\
\hline 19 & & & & & & \\
\hline 20 & & & & & & \\
\hline 21 & & & & & & \\
\hline 22 & & & & & & \\
\hline 23 & & & & & $-.936(-3.74)$ & \\
\hline 24 & & & & & $-1.44(-4.96)$ & \\
\hline 25 & & & & . $.411(2.06)$ & & \\
\hline 26 & & & & & & \\
\hline 27 & & & $-.657(-2.03)$ & & & \\
\hline 28 & & & & & & \\
\hline 29 & & & & & $-1.18(-2.88)$ & \\
\hline 30 & & & & & & $2.49(5.38)$ \\
\hline
\end{tabular}

- positive coefficient

- negative coefficient

"hl10" - "help arrived in 10 minutes or less after the crash" indicator variable

"hg30" - "help arrived in more than 30 minutes after the crash" indicator variable

"hg60" - "help arrived in more than 1 hour after the crash" indicator variable

"car" - "the vehicle at fault is a car" indicator variable

"heavy" - "the vehicle at fault is a truck or a tractor" indicator variable

"moto" - "the vehicle at fault is a motorcycle" indicator variable 
Table B.3 (Continued)

\begin{tabular}{|c|c|c|c|c|c|c|}
\hline \multirow{2}{*}{ \# } & \multicolumn{6}{|c|}{ Coefficient (t-ratio) } \\
\hline & pickup $\left[\mathrm{X}_{25}\right]$ & vage $\left[X_{26}\right]$ & voldg $\left[\mathrm{X}_{26}\right]$ & $\operatorname{v7g}\left[X_{26}\right]$ & $X_{27}$ & Ind $\left[\mathrm{X}_{28}\right]$ \\
\hline 1 & & & & & & \\
\hline 2 & & & & & & \\
\hline 3 & & & .256(4.66) & & $.131(4.84)$ & $-.353(-2.79)$ \\
\hline 4 & & & & & & \\
\hline 5 & & & & & $-2.47(-2.15)$ & \\
\hline 6 & & & & & & \\
\hline 7 & & & & & & $-.667(-3.22)$ \\
\hline 8 & & & & & $-.586(-3.35)$ & \\
\hline 9 & & & & & & \\
\hline 10 & & & & & & \\
\hline 11 & & & & & $.273(2.08)$ & \\
\hline 12 & & & & & & \\
\hline 13 & & & & & & \\
\hline 14 & $942(2.34)$ & & & & & \\
\hline 15 & & $.0411(4.64)$ & & & & \\
\hline 16 & & & & & & \\
\hline 17 & & & & & & \\
\hline 18 & & & & & & \\
\hline 19 & & & & & & \\
\hline 20 & & & & & & \\
\hline 21 & & & & & & \\
\hline 22 & & $.0255(5.39)$ & & & & \\
\hline 23 & & $0.0629(3.29)$ & & & & \\
\hline 24 & & & & & & \\
\hline 25 & & & & & $-.302(-2.24)$ & \\
\hline 26 & & & & & & \\
\hline 21 & & & & & & \\
\hline 28 & & & & & & \\
\hline 29 & & & & & & \\
\hline 30 & & & & $-.724(-2.38)$ & .320(2.34) & \\
\hline
\end{tabular}

- positive coefficient

- negative coefficient

"pickup" - "the vehicle at fault is a pickup" indicator variable

"vage" - "age (in years) of the vehicle at fault" quantitative variable

"voldg" - "the vehicle at fault is more than 7 years old" indicator variable

"v7g" - "age of the vehicle at fault is $>3$ and $\leq 7$ years" indicator variable

" $\mathrm{X}_{27}$ " - "number of occupants in the vehicle at fault" quantitative variable

"Ind" - - "license state of the vehicle at fault is Indiana" indicator variable 
Table B.3 (Continued)

\begin{tabular}{|c|c|c|c|c|c|c|c|}
\hline \multirow{2}{*}{ \# } & \multicolumn{7}{|c|}{ Coefficient (t-ratio) } \\
\hline & $\begin{array}{r}\text { othUS } \\
{\left[\mathrm{X}_{28}\right]} \\
\end{array}$ & $\begin{array}{c}\text { neighs } \\
{\left[\mathrm{X}_{28}\right]}\end{array}$ & w2 [ $\left.\mathrm{X}_{30}\right]$ & $\ln 2\left[X_{30}\right]$ & $\mathrm{r} 22\left[\mathrm{X}_{30}\right]$ & $\begin{array}{l}\mathrm{rmu} 2 \\
{\left[\mathrm{X}_{30}\right]} \\
\end{array}$ & $\begin{array}{c}\text { rmu22 } \\
{\left[\mathrm{X}_{30}\right]} \\
\end{array}$ \\
\hline \multicolumn{8}{|l|}{1} \\
\hline \multirow{2}{*}{\multicolumn{8}{|c|}{$\frac{2}{3}$}} \\
\hline & & & & & & & \\
\hline \multicolumn{8}{|l|}{4} \\
\hline \multicolumn{8}{|c|}{$2.34(2.11)$} \\
\hline 6 & & & & & & $2.28(3.65)$ & \\
\hline \multicolumn{8}{|l|}{7} \\
\hline \multicolumn{8}{|l|}{8} \\
\hline \multicolumn{8}{|l|}{9} \\
\hline \multirow{2}{*}{\multicolumn{8}{|c|}{$\begin{array}{l}10 \\
11\end{array}$}} \\
\hline & & & & & & & \\
\hline \multicolumn{8}{|l|}{12} \\
\hline \multicolumn{8}{|l|}{13} \\
\hline \multicolumn{8}{|l|}{14} \\
\hline 15 & & & & & $-.420(-3.41)$ & & \\
\hline 16 & & & & & & & $-2.07(-2.89$ \\
\hline \multicolumn{8}{|r|}{$-2.01(-<.08$} \\
\hline \multicolumn{8}{|l|}{18} \\
\hline 19 & $929(1.97)$ & & $720(2.27)$ & & & & \\
\hline \multicolumn{8}{|l|}{$\frac{20}{21}$} \\
\hline \multicolumn{8}{|c|}{$.312(5.83)$} \\
\hline \multicolumn{8}{|l|}{23} \\
\hline \multicolumn{8}{|l|}{24} \\
\hline \multicolumn{8}{|l|}{25} \\
\hline \multicolumn{8}{|l|}{26} \\
\hline \multicolumn{8}{|l|}{27} \\
\hline 28 & & .653(3.66) & & & & $.357(2.61)$ & \\
\hline 29 & & & & & & & \\
\hline 30 & & & & & & & \\
\hline
\end{tabular}

- positive coefficient

- negative coefficient

"othUS" - "license state of the vehicle at fault is a U.S. state except Indiana and its neighboring states (IL, KY, OH, MI)" indicator variable

"neighs" - "license state of the vehicle at fault is an Indiana's neighboring state (IL, KY, OH, MI)" indicator variable

"w2" - - "road traveled by the vehicle at fault is two-way" indicator variable

"In2" - "road traveled by the vehicle at fault is two-lane" indicator variable

"r22" - "road traveled by the vehicle at fault is two-lane AND two-way" indicator variable

"rmu2" _ - "road traveled by the vehicle at fault is multi-lane AND undivided two-way" indicator variable

"rmu22" - "road traveled by the vehicle at fault is multi-lane AND undivided two-way left" indicator variable 
Table B.3 (Continued)

\begin{tabular}{|c|c|c|c|c|c|c|}
\hline \multirow{2}{*}{ \# } & \multicolumn{6}{|c|}{ Coefficient (t-ratio) } \\
\hline & priv $\left[\mathrm{X}_{30}\right]$ & stopsig $\left[X_{31}\right]$ & nosig $\left[X_{31}\right]$ & nopass $\left[X_{31}\right]$ & Incontr $\left[X_{31}\right]$ & $\operatorname{sign}\left[X_{31}\right]$ \\
\hline 1 & & $-.441(-2.98)$ & & & & \\
\hline 2 & & & & & & \\
\hline 3 & & & $-.519(-9.34)$ & & & \\
\hline 4 & & & & $1.55(4.73)$ & & \\
\hline 5 & & & & & & \\
\hline 6 & & & & $.613(2.25)$ & & \\
\hline 7 & & & & & & \\
\hline 8 & & & & & & $1.30(3.88)$ \\
\hline 9 & & & $-.600(-2.97)$ & & & \\
\hline$\overline{10}$ & & & & & $.674(2.72)$ & \\
\hline 11 & & & & & & \\
\hline 12 & & & & & & \\
\hline 13 & & $-1.54(-3.25)$ & & & & \\
\hline 14 & & & & & & \\
\hline 15 & & $.996(2.67)$ & & & & \\
\hline 16 & & $-1.32(-2.58)$ & & & & \\
\hline 17 & & & & & & \\
\hline 18 & & & $-.665(-2.63)$ & & & \\
\hline 19 & & & & & & \\
\hline 20 & & & & & & \\
\hline 21 & & $619 / 726)$ & & & & \\
\hline $\begin{array}{l}22 \\
23\end{array}$ & & $-.619(-1.36)$ & & & & \\
\hline 24 & $-.599(-2.09$ & & & & & $.175(2.51)$ \\
\hline 25 & & $-1.66(-2.75)$ & & & & \\
\hline 26 & & & & & & \\
\hline 27 & & & $-.351(-2.04)$ & & & \\
\hline 28 & & & & & & \\
\hline 29 & & & & & & \\
\hline 30 & & & & & & \\
\hline
\end{tabular}

- positive coefficient

- negative coefficient

"priv" _ - "road traveled by the vehicle at fault is a private drive" indicator variable

"stopsig" - "traffic control device for the vehicle at fault is a «stop sign»" indicator variable

"nosig" - "no any traffic control device for the vehicle at fault" indicator variable

"nopass" - "traffic control device for the vehicle at fault is a «no passing zone»" indicator variable

"Incontr" - "traffic control device for the vehicle at fault is a «lane control»" indicator variable

"sign" _ - "traffic control device for the vehicle at fault is any traffic sign" indicator Variable 
Table B.3 (Continued)

\begin{tabular}{|c|c|c|c|c|c|c|}
\hline \multirow{2}{*}{$\#$} & \multicolumn{6}{|c|}{ Coefficient (t-ratio) } \\
\hline & $X_{33}$ & $X_{34}$ & age4 $\left[X_{34}\right]$ & $X_{35}$ & $\operatorname{maxpass}\left[\mathrm{X}_{27}\right]$ & $\mathrm{mm}\left[\mathrm{X}_{35}\right]$ \\
\hline 1 & & & $-.0183(-5.71)$ & & $.132(3.13)$ & \\
\hline 2 & & $-.0581(-8.00)$ & & & & \\
\hline 3 & & $-.0293(-13.1)$ & & $-.248(-4.29)$ & & \\
\hline 4 & & $\mid-.0321(-3.45)$ & & & & \\
\hline 5 & & & & & $2.15(2.61)$ & \\
\hline 6 & & $-.0381(-8.19)$ & & & & \\
\hline 7 & & & & & & \\
\hline 8 & & & & & & \\
\hline 9 & & $-.0157(-4.01)$ & & & & \\
\hline 10 & & & & & & $.569(2.72)$ \\
\hline 11 & & & & & & \\
\hline 12 & & & & & & \\
\hline$\overline{13}$ & $2.74(3.85)$ & $-.0143(-2.75)$ & & & & \\
\hline 14 & & & $-1.84(-2.84)$ & & & \\
\hline 15 & & $-.0307(-9.37)$ & & & & \\
\hline 16 & & & & & & \\
\hline 17 & & & & & & \\
\hline 18 & & $-.0313(3.78)$ & & & & \\
\hline 19 & & $-.0201(-3.52)$ & & & & \\
\hline 20 & & \begin{tabular}{|l|}
$-358(-7.93)$ \\
\end{tabular} & & $-.563(-3.69)$ & & \\
\hline 21 & & & & & & \\
\hline 22 & & 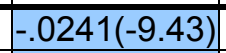 & & $-.211(-3.99)$ & & \\
\hline 23 & & $-.0335(-3.79)$ & & & & \\
\hline 24 & & $-.0367(-14.3)$ & & $-.422(-5.70)$ & & \\
\hline 25 & & & & & & $.498(2.58)$ \\
\hline 26 & & & & & & \\
\hline 27 & & $-.0263(-4.90)$ & & $.333(-2.01)$ & & \\
\hline 28 & & \begin{tabular}{|c|}
$-.0179(-4.69)$ \\
\end{tabular} & & & & \\
\hline 29 & & & & & & \\
\hline 30 & & $-.0203(-2.25)$ & & & & \\
\hline
\end{tabular}

- positive coefficient

- negative coefficient

"X $\mathrm{X}_{33}$ " - "at least one of the vehicles involved was on fire" indicator variable

" $\mathrm{X}_{34}$ " - "age (in years) of the driver at fault" quantitative variable

"age4" - "age of the driver at fault is $\geq 40$ and $<50$ " indicator variable

"X $X_{35}$ " - "gender of the driver at fault: 1 - female, 0 - male" indicator variable

"maxpass" - "the largest number of occupants in all vehicles involved" indicator variable

"mm" _ _ - "two male drivers involved into a two-vehicle accident" indicator variable 
Table B.4 Binary logit models for 2006 accident causation

\begin{tabular}{|c|c|c|c|c|c|c|c|c|}
\hline \multirow{2}{*}{ \# } & & \multirow{2}{*}{\multicolumn{2}{|c|}{ Model name }} & \multicolumn{2}{|c|}{ Log-likelihood } & \multirow{2}{*}{$R^{2}$} & \multicolumn{2}{|c|}{ Coefficient (t-ratio) } \\
\hline & & & & model & restricted $^{*}$ & & $\mathrm{X}_{29}$ & constant \\
\hline $1^{* *}$ & \multirow{4}{*}{ 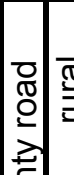 } & & (car/SUV)+(car/SUV) & -342.72 & -41.06 & .164 & & \\
\hline $2 a$ & & $\bar{\pi}$ & (car)+(truck) & -18.849 & -26.763 & .296 & & $-5.12(-5.10)$ \\
\hline $2 b$ & & & (SUV)+(truck) & -5.0592 & -10.270 & .507 & & \\
\hline 3 & & & one vehicle & -1204.3 & -1438.2 & .163 & & \\
\hline 4 & & $(\mathrm{car} / \mathrm{SUV})+(\mathrm{car} / \mathrm{SUV})$ & -227.21 & -269.91 & .158 & & $-2.63(-12.2)$ \\
\hline 5 & & & (car/SUV)+(truck) & -15.788 & -18.893 & .164 & & $-3.54(-4.94)$ \\
\hline 6 & & & one vehicle & -256.10 & -300.36 & .147 & & \\
\hline $7 a$ & & & (car)+(car) & -85.811 & -118.56 & .276 & & $-1.50(-3.53)$ \\
\hline $7 b$ & & & (car)+(SUV) & -127.43 & -178.37 & .286 & & $-2.14(-3.06)$ \\
\hline $7 c$ & & & (SUV)+(SUV) & -50.205 & -78.501 & .360 & & $-1.98(-4.10)$ \\
\hline 8 & $\stackrel{0}{\frac{\pi}{\sigma}}$ & & (car/SUV)+(truck) & -222.35 & -287.72 & .227 & & $-1.08(-2.67)$ \\
\hline 9 & $\frac{\pi}{\omega}$ & & one vehicle & -918.40 & -1435.7 & .360 & $-.0439(-5.72)$ & $3.59(6.96)$ \\
\hline 10 & $\stackrel{\bar{\Phi}}{=}$ & \multirow{4}{*}{ 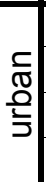 } & $(\mathrm{car} / \mathrm{SUV})+(\mathrm{car} / \mathrm{SUV})$ & -508.16 & -603.72 & .158 & & $-2.77(-6.43)$ \\
\hline $11 \mathrm{a}$ & & & (car)+(truck) & -116.08 & -159.73 & .273 & & $-1.31(-3.03)$ \\
\hline $11 b$ & & & (SUV)+(truck) & -32.424 & -65.943 & .508 & & $-4.48(-3.00)$ \\
\hline 12 & & & one vehicle & -136.07 & -169.56 & .198 & & $-1.04(-2.03)$ \\
\hline 13 & \multirow{3}{*}{\multicolumn{2}{|c|}{\begin{tabular}{l|l}
0 \\
\multirow{2}{*}{} \\
0
\end{tabular}}} & $($ car/SUV)+(car/SUV) & -774.29 & -863.45 & .103 & & $-1.68(-7.77)$ \\
\hline 14 & & & (car/SUV)+(truck) & -92.951 & -114.16 & .186 & & $-2.63(-6.19)$ \\
\hline 15 & & & one vehicle & -1616.3 & -2084.9 & .225 & $-.0373(-5.34)$ & $1.07(2.68)$ \\
\hline 16 & $\stackrel{\oplus}{=}$ & \multirow{3}{*}{$\begin{array}{l}\frac{c}{\pi} \\
\frac{\pi}{3} \\
\frac{0}{J}\end{array}$} & $($ car/SUV)+(car/SUV) & -850.84 & -952.04 & .106 & $.0277(3.47)$ & $-2.50(-6.45)$ \\
\hline 17 & 岕. & & (car/SUV)+(truck) & -81.158 & -93.656 & .133 & & $-3.76(-11.3)$ \\
\hline 18 & & & one vehicle & -358.35 & -441.38 & .188 & & $.615(2.03)$ \\
\hline 19 & & \multirow{4}{*}{ 艿 } & $(\mathrm{car} / \mathrm{SUV})+(\mathrm{c}$ & -211.60 & -227.51 & .070 & & $-3.41(-18.0)$ \\
\hline $20 a$ & & & (car)+ & -13.637 & -16.670 & .182 & & $(-4.32)$ \\
\hline $20 b$ & 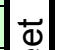 & & $($ SUV)+ & -11.753 & -11.753 & .000 & & $(-4.87)$ \\
\hline 21 & $\stackrel{\Phi}{=}$ & & one vehicle & -597.08 & -694.78 & .141 & & $-1.63(-6.62)$ \\
\hline 22 & का & \multirow{4}{*}{$\begin{array}{l}\frac{\sqrt{\pi}}{\pi} \\
\frac{0}{3}\end{array}$} & (car/SUV)+(car/SUV) & -2265.6 & -2584.8 & .123 & & $-3.02(-12.6)$ \\
\hline $23 a$ & U) & & (car)+(truck) & -93.362 & -115.47 & .191 & & $-5.49(-10.0)$ \\
\hline $23 b$ & & & (SUV)+(truck) & -29.107 & -40.704 & .285 & & $-6.80(-6.78)$ \\
\hline 24 & & & one vehicle & -2597.7 & -3024.6 & .141 & & $-.556(-4.78)$ \\
\hline 25 & & \multirow{3}{*}{ 离 } & $($ (car/SUV)+(car/SUV) & -456.42 & -499.97 & .087 & & \\
\hline 26 & & & (car/SUV)+(truck) & -80.524 & -102.56 & .215 & & $-4.55(-8.95)$ \\
\hline 27 & $\stackrel{\frac{0}{3}}{3}$ & & one vehicle & \begin{tabular}{|l|}
-708.44 \\
\end{tabular} & -977.40 & .275 & & $-1.60(-5.96)$ \\
\hline 28 & 은 & \multirow{4}{*}{ 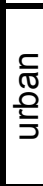 } & $(\mathrm{car} / \mathrm{SUV})+(\mathrm{car} / \mathrm{SUV})$ & -834.94 & -901.81 & .074 & & $-1.68(-6.19)$ \\
\hline $29 a$ & 包 & & (car)+(truck) & -46.549 & -51.986 & .105 & & $-3.60(-10.6)$ \\
\hline $29 b$ & & & (SUV)+(truck) & -11.446 & -11.446 & .000 & & $-4.72(-6.64)$ \\
\hline 30 & & & one vehicle & -250.31 & -315.51 & .207 & & $-1.71(-5.00)$ \\
\hline $\begin{array}{l}\ldots \\
* \\
* *\end{array}$ & \multicolumn{8}{|c|}{$\begin{array}{l}\text { - positive coefficient } \ldots . . . \text { - negative coefficient } \\
\text { - restricted log-likelihood found by setting all coefficients except intercepts to zero } \\
\text { - models are estimated by using procedure } A \text { on page } 32 \text {, except the models } \\
\text { marked by bold numbers and estimated by using procedure B on page } 33\end{array}$} \\
\hline
\end{tabular}

" $\mathrm{X}_{29}$ " - "posted speed limit (if the same for all vehicles involved)" quantitative variable "constant" - "constant term (intercept)" quantitative variable 
Table B.4 (Continued)

\begin{tabular}{|c|c|c|c|c|c|c|}
\hline \multirow{2}{*}{ \# } & \multicolumn{6}{|c|}{ Coefficient (t-ratio) } \\
\hline & nigh $\left[X_{5}\right]$ & dayt $\left[X_{5}\right]$ & lunch $\left[X_{5}\right]$ & light $\left[X_{14}\right]$ & $\operatorname{dark}\left[\mathrm{X}_{14}\right]$ & $\operatorname{day}\left[\mathrm{X}_{14}\right]$ \\
\hline \multicolumn{7}{|l|}{$1^{* \star}$} \\
\hline \multicolumn{7}{|l|}{$2 a$} \\
\hline \multicolumn{7}{|l|}{$2 b$} \\
\hline 3 & & & & & & $.368(3.59)$ \\
\hline \multicolumn{7}{|l|}{4} \\
\hline \multicolumn{7}{|l|}{5} \\
\hline \multicolumn{7}{|l|}{6} \\
\hline \multicolumn{7}{|l|}{$7 a$} \\
\hline \multirow{2}{*}{\multicolumn{7}{|c|}{$\begin{array}{ll}7 b \\
7 c\end{array}$}} \\
\hline & & & & & & \\
\hline \multicolumn{7}{|l|}{8} \\
\hline \multicolumn{7}{|c|}{$-825(-4.07)$} \\
\hline 10 & \multicolumn{6}{|r|}{$-.381(-2.15)$} \\
\hline $11 \mathrm{a}$ & $1.48(2.73)$ & & & & & \\
\hline \multirow{2}{*}{\multicolumn{7}{|c|}{$-2.64(-3.47)$}} \\
\hline & & & & & & \\
\hline \multicolumn{7}{|l|}{13} \\
\hline \multicolumn{7}{|c|}{$1.06(2.36)$} \\
\hline \multicolumn{7}{|c|}{$.792(8.08)$} \\
\hline 16 & $1.41(3.56)$ & & & & & \\
\hline \multicolumn{7}{|c|}{$1.1+10.00$} \\
\hline \multicolumn{7}{|c|}{$.511(2.37)$} \\
\hline \multicolumn{7}{|l|}{19} \\
\hline \multirow{2}{*}{\multicolumn{7}{|c|}{$\frac{20 a}{20 b}$}} \\
\hline & & & & & & \\
\hline \multicolumn{7}{|c|}{$.527(3.37)$} \\
\hline 22 & & $-.216(-2.45)$ & & & & \\
\hline \multicolumn{7}{|l|}{$23 \mathrm{a}$} \\
\hline \multicolumn{7}{|l|}{$23 b$} \\
\hline \multicolumn{7}{|l|}{24} \\
\hline \multirow{2}{*}{\multicolumn{7}{|c|}{$\frac{25}{76}$}} \\
\hline & & & & & & \\
\hline 27 & & & & & $-.721(-4.92)$ & \\
\hline 28 & & & & $-.609(-2.91)$ & & \\
\hline $29 a$ & & & & & & \\
\hline $29 b$ & & & & & & \\
\hline 30 & & & & & & \\
\hline$\ldots$ & positive & ficient & $\ldots$ & - negative & oefficient. & \\
\hline
\end{tabular}

"nigh" - "late night hours: from 1:00 to 5:00" indicator variable

"dayt" - "day hours: from 9:00 to 17:00" indicator variable

"lunch" - "lunch hours: 11:00 to 14:00" indicator variable

"light" - "daylight time OR street lights lit up during dark time" indicator variable

"dark" - - "dark time with no street lights" indicator variable

"day" _ - "daylight time" indicator variable 
Table B.4 (Continued)

\begin{tabular}{|c|c|c|c|c|c|c|}
\hline \multirow{2}{*}{ \# } & \multicolumn{6}{|c|}{ Coefficient (t-ratio) } \\
\hline & $\begin{array}{c}\text { darklamp } \\
{\left[\mathrm{X}_{14}\right]}\end{array}$ & precip $\left[\mathrm{X}_{15}\right]$ & snow $\left[X_{15}\right]$ & dry $\left[\mathrm{X}_{16}\right]$ & slush $\left[\mathrm{X}_{16}\right]$ & ice $\left[X_{16}\right]$ \\
\hline $1^{\text {**}}$ & & & & $-1.88(-8.85)$ & & \\
\hline $2 a$ & & $3.68(3.29)$ & & & & \\
\hline \multicolumn{7}{|l|}{$2 b$} \\
\hline 3 & & & & $-1.28(-12.6)$ & & \\
\hline 4 & & & & $-1.90(-6.62)$ & & \\
\hline \multicolumn{7}{|l|}{5} \\
\hline 6 & & & $1.34(2.95)$ & $-.730(-3.18)$ & & \\
\hline$\overline{7 a}$ & & & & $-2.77(-6.39)$ & & \\
\hline $7 b$ & $1.42(3.10)$ & & & $-2.34(-7.03)$ & & \\
\hline $7 c$ & & & $3.49(3.00)$ & $-2.09(-3.70)$ & & \\
\hline 8 & & $1.02(2.34)$ & & \begin{tabular}{|l|}
$-1.37(-3.04)$ \\
\end{tabular} & & \\
\hline 9 & & $.753(4.35)$ & & $-2.57(-12.4)$ & & \\
\hline 10 & & $.718(2.30)$ & & $-1.21(-3.91)$ & & \\
\hline $11 a$ & & & & $-2.34(-6.59)$ & & \\
\hline $11 b$ & & & $6.96(4.53)$ & & & \\
\hline 12 & & $1.96(5.97)$ & & & & \\
\hline 13 & & & & $-1.70(-11.7)$ & & \\
\hline 14 & & & & $-1.44(-3.39)$ & & \\
\hline 15 & & $.342(2.56)$ & & $-1.39(-10.2)$ & & \\
\hline 16 & & & & $-1.62(-11.4)$ & & \\
\hline 17 & & & & & & $4.24(3.41)$ \\
\hline 18 & & & & $-1.46(-7.22)$ & & \\
\hline 19 & & $1.13(3.93)$ & & & & \\
\hline \multicolumn{7}{|l|}{$20 a$} \\
\hline \multicolumn{7}{|l|}{$20 \mathrm{~b}$} \\
\hline 21 & & & $.904(2.42)$ & & $1.22(3.29)$ & \\
\hline 22 & & $.305(2.44)$ & & $-1.58(-12.0)$ & & \\
\hline \multirow{2}{*}{\multicolumn{7}{|c|}{$23 a$}} \\
\hline & \multicolumn{6}{|c|}{$23 b$} \\
\hline 24 & & & & $-.985(-13.3)$ & & \\
\hline 25 & & & & $-1.57(-8.64)$ & & \\
\hline 26 & & $2.44(5.48)$ & & & & \\
\hline 27 & & $.468(2.48)$ & & $-1.87(-8.86)$ & & \\
\hline 28 & & & & $-1.41(-9.68)$ & & \\
\hline $29 a$ & & & & & $2.76(2.62)$ & \\
\hline $29 b$ & & & & & & \\
\hline 30 & & $1.99(8.17)$ & & & & \\
\hline
\end{tabular}

- positive coefficient

....- negative coefficient.

"darklamp" - "dark time with street lights on" indicator variable

"precip" - - "precipitation: rain OR snow OR sleet OR hail OR freezing rain" indicator variable

"snow" _ - "snowing weather" indicator variable

"dry" _ - "roadway surface is dry" indicator variable

"slush" _ - "roadway surface is covered by snow/slush" indicator variable

"ice" _ _ _ " "roadway surface is icy" indicator variable 
Table B.4 (Continued)

\begin{tabular}{|c|c|c|c|c|c|c|}
\hline \multirow{2}{*}{ \# } & \multicolumn{6}{|c|}{ Coefficient (t-ratio) } \\
\hline & $\operatorname{driv}\left[\mathrm{X}_{17}\right]$ & wall $\left[\mathrm{X}_{17}\right]$ & nojun $\left[\mathrm{X}_{18}\right]$ & $\operatorname{ramp}\left[\mathrm{X}_{18}\right]$ & way4 $\left[\mathrm{X}_{18}\right]$ & $\mathrm{T}\left[\mathrm{X}_{18}\right]$ \\
\hline 1** & & & & & $-.786(-2.54)$ & \\
\hline \multicolumn{7}{|l|}{$2 a$} \\
\hline $2 b$ & & & & & & \\
\hline 3 & & $1.64(2.35)$ & $-.639(-5.53)$ & & & \\
\hline \multicolumn{7}{|l|}{4} \\
\hline \multicolumn{7}{|l|}{5} \\
\hline 6 & & $2.33(2.29)$ & & & & $.729(-3.18)$ \\
\hline \multicolumn{7}{|l|}{$7 a$} \\
\hline $7 b$ & & & $1.66(2.82)$ & & & \\
\hline \multicolumn{7}{|l|}{$7 c$} \\
\hline \multicolumn{7}{|l|}{8} \\
\hline 9 & & $.302(2.18)$ & $-1.20(-5.30)$ & & & \\
\hline 10 & & $.637(2.64)$ & & & & \\
\hline 11a & & & & & & \\
\hline $11 b$ & & $3.16(2.10)$ & & & & \\
\hline \multicolumn{7}{|l|}{12} \\
\hline$\overline{13}$ & & & $.357(2.40)$ & & & \\
\hline 14 & $1.17(2.45)$ & & & & & \\
\hline 15 & & & & $.990(2.36)$ & & \\
\hline \multirow{2}{*}{\multicolumn{7}{|c|}{16}} \\
\hline & & & & & & \\
\hline \multicolumn{7}{|l|}{18} \\
\hline \multicolumn{7}{|l|}{19} \\
\hline \multicolumn{7}{|l|}{$20 \mathrm{a}$} \\
\hline \multicolumn{7}{|l|}{$20 b$} \\
\hline 21 & & & & $1.25(2.10)$ & & \\
\hline 22 & $.180(2.01)$ & & & & $-.234(-2.52)$ & \\
\hline $23 a$ & & & & & & $1.31(2.72)$ \\
\hline \multicolumn{7}{|l|}{ 23b } \\
\hline 24 & & & & & & $.401(4.14)$ \\
\hline \multirow{2}{*}{\multicolumn{7}{|c|}{$\frac{25}{26}$}} \\
\hline & & & \\
\hline 27 & $-.765(-3.56)$ & & & & & \\
\hline 28 & & & $.307(2.13)$ & & & \\
\hline \multicolumn{7}{|l|}{$29 a$} \\
\hline \multirow{2}{*}{\multicolumn{7}{|c|}{$29 b$}} \\
\hline & & & & & & \\
\hline.. & - positive co & ficient & & - negative & coefficient. & \\
\hline
\end{tabular}

"driv" - "road median is a drivable" indicator variable

"wall" - "road median is a barrier wall" indicator variable

"nojun" - "no road junction at the accident location" indicator variable

"ramp" - "accident location is near or on a ramp" indicator variable

"way4" - "accident location is at a 4-way intersection" indicator variable

"T" - "accident location is at a T-intersection" indicator variable 
Table B.4 (Continued)

\begin{tabular}{|c|c|c|c|c|c|c|}
\hline \multirow{2}{*}{ \# } & \multicolumn{6}{|c|}{ Coefficient (t-ratio) } \\
\hline & $Y\left[X_{18}\right]$ & $\operatorname{int}\left[\mathrm{X}_{18}\right]$ & curve $\left[\mathrm{X}_{19}\right]$ & $\operatorname{sg}\left[X_{19}\right]$ & sl $\left[X_{19}\right]$ & $\operatorname{str}\left[X_{19}\right]$ \\
\hline \multicolumn{7}{|l|}{$1^{\star \star}$} \\
\hline \multicolumn{7}{|l|}{$2 a$} \\
\hline \multicolumn{7}{|l|}{$2 b$} \\
\hline 3 & & & $1.05(9.61)$ & & & \\
\hline \multicolumn{7}{|l|}{4} \\
\hline \multicolumn{7}{|l|}{5} \\
\hline 6 & & & & & & $-.857(-3.73)$ \\
\hline \multicolumn{7}{|l|}{$7 a$} \\
\hline \multicolumn{7}{|l|}{$7 b$} \\
\hline \multirow{2}{*}{\multicolumn{7}{|c|}{$\frac{7 c}{8}$}} \\
\hline & & & & & & \\
\hline 9 & & & & & $-.418(-3.33)$ & \\
\hline \multicolumn{2}{|c|}{$-.410(-0.00)$} & & & & & \\
\hline \multicolumn{7}{|c|}{ 11a } \\
\hline \multicolumn{7}{|c|}{$11 b$} \\
\hline 12 & & & & & & $-1.62(-4.84)$ \\
\hline \multicolumn{7}{|l|}{13} \\
\hline 14 & $3.27(3.48)$ & & & $1.17(2.71)$ & & \\
\hline 15 & & & & & & $-1.05(-10.6)$ \\
\hline \multicolumn{7}{|l|}{16} \\
\hline \multicolumn{7}{|l|}{17} \\
\hline 18 & & & & & & $-1.18(-5.70)$ \\
\hline 19 & & & $1.34(3.18)$ & & & \\
\hline \multicolumn{7}{|c|}{$20 \mathrm{a}$} \\
\hline \multicolumn{7}{|c|}{$20 b$} \\
\hline 21 & & & $1.15(7.43)$ & & & \\
\hline 22 & & & $.898(5.40)$ & & & \\
\hline $23 a$ & & & $1.90(2.84)$ & & & \\
\hline \multicolumn{7}{|c|}{$23 b$} \\
\hline 24 & & & $1.11(13.9)$ & & & \\
\hline 25 & & & & & & $-.640(-2.84)$ \\
\hline \multicolumn{7}{|l|}{26} \\
\hline 27 & $2.15(3.85)$ & & $1.00(6.70)$ & & & \\
\hline \multicolumn{7}{|l|}{28} \\
\hline \multicolumn{7}{|l|}{$29 a$} \\
\hline $29 b$ & & & & & & \\
\hline 30 & & $1.45(2.24)$ & $.813(2.91)$ & & & \\
\hline
\end{tabular}

- positive coefficient

.... - negative coefficient.

"Y" - "accident location is at a Y-intersection" indicator variable

"int" - "accident location is near or on an interchange" indicator variable

"curve" - "road is at curve" indicator variable

"sg" - "road is straight AND at grade" indicator variable

"sl" - "road is straight AND level" indicator variable

"str" _ - "road is straight" indicator variable 
Table B.4 (Continued)

\begin{tabular}{|c|c|c|c|c|c|c|}
\hline \multirow{2}{*}{ \# } & \multicolumn{6}{|c|}{ Coefficient (t-ratio) } \\
\hline & $\mathrm{Cl}\left[\mathrm{X}_{19}\right]$ & $\operatorname{cg}\left[X_{19}\right]$ & $\operatorname{lev}\left[X_{19}\right]$ & $\operatorname{grd}\left[\mathrm{X}_{19}\right]$ & $\mathrm{h} \mid 5\left[\mathrm{X}_{22}\right]$ & $\mathrm{hl} 10\left[\mathrm{X}_{22}\right]$ \\
\hline \multicolumn{7}{|l|}{$1^{\star \star}$} \\
\hline \multicolumn{7}{|l|}{$2 a$} \\
\hline \multirow{2}{*}{\multicolumn{7}{|c|}{$\begin{array}{c}2 \boldsymbol{2 b} \\
3\end{array}$}} \\
\hline & & & & & & \\
\hline 4 & $1.32(3.09)$ & & & & & \\
\hline \multicolumn{7}{|l|}{5} \\
\hline \multicolumn{7}{|l|}{6} \\
\hline $7 a$ & $1.39(2.17)$ & & & & & \\
\hline $7 b$ & $1.61(2.93)$ & & & & & \\
\hline \multirow{2}{*}{\multicolumn{7}{|c|}{$\frac{7 c}{8}$}} \\
\hline & & & & & & \\
\hline \multicolumn{4}{|c|}{$324(206)$} & & $.324(2.06)$ & \\
\hline \multicolumn{7}{|l|}{10} \\
\hline $11 a$ & & & & & & \\
\hline \multicolumn{7}{|c|}{$11 b$} \\
\hline \multicolumn{7}{|l|}{12} \\
\hline 13 & & $.937(3.86)$ & & & & \\
\hline \multicolumn{7}{|l|}{14} \\
\hline \multicolumn{7}{|l|}{15} \\
\hline 16 & & & $-.490(-2.92)$ & & & \\
\hline 17 & & & & $1.11(2.28)$ & & \\
\hline \multicolumn{7}{|l|}{18} \\
\hline \multirow{2}{*}{\multicolumn{7}{|c|}{19}} \\
\hline & & & & & & \\
\hline \multicolumn{7}{|l|}{$20 b$} \\
\hline 21 & & & & & & $.403(2.61)$ \\
\hline \multicolumn{7}{|l|}{22} \\
\hline \multicolumn{7}{|l|}{ 23a } \\
\hline \multirow{2}{*}{\multicolumn{7}{|c|}{$\frac{200}{24}$}} \\
\hline & & & & & & \\
\hline \multicolumn{7}{|l|}{25} \\
\hline 26 & & & & & & $.957(2.04)$ \\
\hline 27 & & & & & & $.630(4.39)$ \\
\hline \multicolumn{7}{|l|}{28} \\
\hline \multirow{2}{*}{\multicolumn{7}{|c|}{$29 a$}} \\
\hline & & & & & & \\
\hline 30 & & & & & & \\
\hline
\end{tabular}

"cl" _ _ "road is at-curve AND level" indicator variable

"cg" - "road is at-curve AND grade" indicator variable

"lev" _ - "road is 'at-curve or straight' AND level" indicator variable

"grd" - - "road is 'at-curve or straight' AND grade" indicator variable

"hl5" _ - "help arrived in 5 minutes or less after the crash" indicator variable

"hl10" - "help arrived in 10 minutes or less after the crash" indicator variable 
Table B.4 (Continued)

\begin{tabular}{|c|c|c|c|c|c|c|}
\hline \multirow{2}{*}{ \# } & \multicolumn{6}{|c|}{ Coefficient (t-ratio) } \\
\hline & $\operatorname{car}\left[\mathrm{X}_{25}\right]$ & heavy $\left[X_{25}\right]$ & $\operatorname{van}\left[X_{25}\right]$ & truck3 $\left[\mathrm{X}_{25}\right]$ & $\operatorname{trac} 1\left[\mathrm{X}_{25}\right]$ & vage $\left[X_{26}\right]$ \\
\hline \multicolumn{7}{|l|}{$1^{* *}$} \\
\hline \multicolumn{7}{|l|}{$2 a$} \\
\hline \multirow{2}{*}{\multicolumn{7}{|c|}{$\frac{2 b}{3}$}} \\
\hline & & & & & & \\
\hline \multicolumn{7}{|l|}{4} \\
\hline 5 & & & $2.85(2.01)$ & & & \\
\hline \multicolumn{7}{|l|}{6} \\
\hline \multirow{2}{*}{\multicolumn{7}{|c|}{$7 a$}} \\
\hline & & & & & & \\
\hline \multicolumn{7}{|l|}{$7 c$} \\
\hline 8 & & $-1.18(-4.12)$ & & & & \\
\hline 9 & & $-.509(-2.61)$ & & & & \\
\hline \multicolumn{7}{|c|}{$40,12.01$} \\
\hline $11 \mathrm{a}$ & & & & & $-1.28(-2.77)$ & \\
\hline \multirow{2}{*}{\multicolumn{7}{|c|}{$\frac{11 b}{12}$}} \\
\hline & & & & & & \\
\hline \multicolumn{7}{|l|}{13} \\
\hline 14 & & & & $1.33(1.98)$ & & \\
\hline 15 & & & $-.733(-2.65)$ & & & $.0184(2.03)$ \\
\hline \multirow{2}{*}{\multicolumn{7}{|c|}{$\frac{16}{17}$}} \\
\hline & & & & & & \\
\hline \multicolumn{7}{|l|}{18} \\
\hline \multicolumn{7}{|l|}{19} \\
\hline \multicolumn{7}{|l|}{$20 \mathrm{a}$} \\
\hline \multirow{2}{*}{\multicolumn{7}{|c|}{$\frac{20 b}{21}$}} \\
\hline \multirow{2}{*}{\multicolumn{6}{|c|}{$.0277(3.44)$}} & \\
\hline & & & & & & $\overline{.0277(3.44)}$ \\
\hline $23 a$ & $1.71(3.17)$ & & & & & \\
\hline \multicolumn{7}{|c|}{\begin{tabular}{l|ll}
$23 b$ & $1.1(1)$ \\
\end{tabular}} \\
\hline 24 & & $-1.17(-3.74)$ & & & & \\
\hline \multirow{2}{*}{\multicolumn{7}{|c|}{$\frac{25}{26}$}} \\
\hline & & & & & & \\
\hline 27 & & & & & $1.04(3.77)$ & \\
\hline \multicolumn{7}{|l|}{28} \\
\hline \\
\hline \multirow{2}{*}{\multicolumn{7}{|c|}{$29 b$}} \\
\hline & & & & & & \\
\hline$\ldots$ & - nositi & fficient & & - negative & oefficient. & \\
\hline
\end{tabular}

"car" _ - "the vehicle at fault is a car" indicator variable

"heavy" - "the vehicle at fault is a truck or a tractor" indicator variable

"van" - "the vehicle at fault is a van" indicator variable

"track3" - "the vehicle at fault is a truck (single 3 or more axes)" indicator variable

"trac1" - "the vehicle at fault is a tractor/one semi trailer" indicator variable

"vage" - "age (in years) of the vehicle at fault" quantitative variable 
Table B.4 (Continued)

\begin{tabular}{|c|c|c|c|c|c|c|}
\hline \multirow{2}{*}{$\#$} & \multicolumn{6}{|c|}{ Coefficient (t-ratio) } \\
\hline & voldg $\left[\mathrm{X}_{26}\right]$ & $X_{27}$ & Ind $\left[\mathrm{X}_{28}\right]$ & neighs $\left[\mathrm{X}_{28}\right]$ & neighc $\left[\mathrm{X}_{28}\right]$ & w2 $\left[X_{30}\right]$ \\
\hline \multicolumn{7}{|l|}{$1^{* \star}$} \\
\hline \multicolumn{7}{|l|}{$2 a$} \\
\hline \multirow{2}{*}{\multicolumn{7}{|c|}{$4.40(2.10)$}} \\
\hline & & & & & & \\
\hline \multicolumn{7}{|l|}{4} \\
\hline \multicolumn{7}{|l|}{5} \\
\hline 6 & $.522(2.49)$ & & & & & \\
\hline \multicolumn{7}{|l|}{$7 a$} \\
\hline $7 b$ & & $-.527(-2.07)$ & & & & \\
\hline $7 c$ & $1.33(2.42)$ & & & & & \\
\hline \multicolumn{7}{|c|}{$\ldots-1-\cdots-1$} \\
\hline \multicolumn{7}{|l|}{9} \\
\hline \multicolumn{7}{|l|}{10} \\
\hline $11 \mathrm{a}$ & & & $.910(2.08)$ & & & \\
\hline \multicolumn{7}{|c|}{$11 b$} \\
\hline \multirow{2}{*}{\multicolumn{6}{|c|}{$\frac{12}{13}$}} & \\
\hline \multirow{2}{*}{\multicolumn{7}{|c|}{14}} \\
\hline & & & & & & \\
\hline \multicolumn{7}{|l|}{15} \\
\hline \multicolumn{7}{|l|}{16} \\
\hline \multirow{2}{*}{\multicolumn{7}{|c|}{$\frac{17}{18}$}} \\
\hline & & & & & & \\
\hline \multicolumn{7}{|l|}{19} \\
\hline \multicolumn{7}{|c|}{$20 \mathrm{a}$} \\
\hline \multirow{2}{*}{\multicolumn{7}{|c|}{$\frac{20 b}{21}$}} \\
\hline & & & & & & \\
\hline 22 & & & & & & $.601(3.57)$ \\
\hline \multicolumn{7}{|c|}{$23 a$} \\
\hline \multicolumn{7}{|c|}{$23 b$} \\
\hline 24 & & $.0805(3.26)$ & & & & \\
\hline 25 & & & $-.734(-3.35)$ & & & \\
\hline \multicolumn{7}{|l|}{26} \\
\hline \multicolumn{7}{|l|}{$\frac{27}{30}$} \\
\hline \multirow{2}{*}{\multicolumn{7}{|c|}{$\begin{array}{c}\mathbf{2 8} \\
29 a\end{array}$}} \\
\hline & & & & & & \\
\hline $29 b$ & & & & & & \\
\hline 30 & & & & $.959(2.98)$ & & \\
\hline
\end{tabular}

- positive coefficient

.... - negative coefficient.

"voldg" - "the vehicle at fault is more than 7 years old" indicator variable

"X $\mathrm{X}_{27 "} \quad$ - "number of occupants in the vehicle at fault" quantitative variable

"Ind" _ - "license state of the vehicle at fault is Indiana" indicator variable

"neighs" - "license state of the vehicle at fault is Indiana's neighboring state (IL, KY, OH, MI)" indicator variable

"neighc" - "license state of the vehicle at fault is from Canada, Mexico, or US territories" indicator variable

"w2" - "road traveled by the vehicle at fault is two-way" indicator variable 
Table B.4 (Continued)

\begin{tabular}{|c|c|c|c|c|c|c|}
\hline \multirow{2}{*}{ \# } & \multicolumn{6}{|c|}{ Coefficient (t-ratio) } \\
\hline & $\mathrm{r} 11\left[\mathrm{X}_{30}\right]$ & r21 $\left[X_{30}\right]$ & rmd2 $\left[X_{30}\right]$ & priv $\left[X_{30}\right]$ & $\mathrm{w} 1\left[\mathrm{X}_{30}\right]$ & stopsig $\left[X_{31}\right]$ \\
\hline $1^{* *}$ & & & & & & $-.658(-2.23)$ \\
\hline $2 a$ & & & & & & \\
\hline $2 b$ & & & & & & \\
\hline 3 & & & & & & \\
\hline 4 & & & & & & \\
\hline 5 & & & & & & \\
\hline 6 & & & & & & \\
\hline $7 a$ & & & & & $-2.67(-2.49)$ & \\
\hline $7 b$ & & & & & & \\
\hline $7 c$ & & & & & & \\
\hline 8 & & & & & $-1.63(-2.59)$ & \\
\hline 9 & & & & & & \\
\hline 10 & & & $.664(2.31)$ & & & \\
\hline 11a & & & & & & \\
\hline 11b & $4.12(2.51)$ & & & & & \\
\hline 12 & & & & & & \\
\hline 13 & & & & & & \\
\hline 14 & & & & & & \\
\hline 15 & & & & & & \\
\hline 16 & & & & & & \\
\hline 17 & & & & & & \\
\hline 18 & & & & & & \\
\hline 19 & & & & & & $-1.64(-2.26)$ \\
\hline $20 \mathrm{a}$ & & & & & & \\
\hline $20 \mathrm{~b}$ & & & & & & \\
\hline 21 & & & & & & \\
\hline 22 & & & & & & $-.458(-3.12)$ \\
\hline $23 \mathrm{a}$ & & & & & & \\
\hline $23 b$ & & & & & & \\
\hline 24 & & & & $-.611(-3.14)$ & & \\
\hline 25 & & & & & & \\
\hline 26 & & & & & & \\
\hline 27 & & & & & & \\
\hline 28 & & & & & & \\
\hline $29 a$ & & $2.22(2.22)$ & & & & \\
\hline $29 b$ & & & & & & \\
\hline 30 & & & & & & \\
\hline
\end{tabular}

"r11" - "road traveled by the vehicle at fault is one-lane \& one-way" indicator var.

"r21" _ - "road traveled by the veh. at fault is two-lanes AND one-way" indic. var.

"rmd2" - "road traveled by the vehicle at fault is multy-lane divided 3 or more AND two-way" indicator variable

"priv" _ - "road traveled by the vehicle at fault is private drive" indicator variable

"w1" _ _road traveled by the vehicle at fault is one-way" indicator variable

"stopsig" - "traffic control device for the vehicle at fault is «stop sign»" indicator var. 
Table B.4 (Continued)

\begin{tabular}{|c|c|c|c|c|c|c|}
\hline \multirow{2}{*}{ \# } & \multicolumn{6}{|c|}{ Coefficient (t-ratio) } \\
\hline & nopass $\left[X_{31}\right]$ & Incontr $\left[\mathrm{X}_{31}\right]$ & $\mathrm{fl}\left[\mathrm{X}_{31}\right]$ & $\mathrm{X}_{33}$ & $X_{34}$ & age1 $\left[\mathrm{X}_{34}\right]$ \\
\hline $1^{\text {**}}$ & & & & & $-.0213(-4.20)$ & \\
\hline \multicolumn{7}{|l|}{$2 a$} \\
\hline \multicolumn{7}{|l|}{$2 b$} \\
\hline 3 & $.501(3.69)$ & & & & $-.0391(-11.3)$ & \\
\hline \multicolumn{7}{|l|}{4} \\
\hline \multicolumn{7}{|l|}{5} \\
\hline 6 & & & & & $-.448(-5.98)$ & \\
\hline \multicolumn{7}{|l|}{$7 a$} \\
\hline \multicolumn{7}{|l|}{$7 b$} \\
\hline \multicolumn{7}{|l|}{$7 c$} \\
\hline \multicolumn{7}{|l|}{8} \\
\hline 9 & & & & & $-.0182(-4.10)$ & \\
\hline \multicolumn{7}{|l|}{10} \\
\hline \multirow{2}{*}{\multicolumn{7}{|c|}{$11 \mathrm{a}$}} \\
\hline \multicolumn{2}{|l|}{$11 b$} & & & & & \\
\hline 12 & & $1.09(3.01)$ & & & $-.0229(-2.07)$ & \\
\hline \multicolumn{7}{|l|}{$\overline{13}$} \\
\hline \multicolumn{7}{|l|}{14} \\
\hline 15 & & & & & $-.0346(-9.64)$ & \\
\hline 16 & & & & & $-.0201(-4.56)$ & \\
\hline \multicolumn{7}{|c|}{, } \\
\hline 18 & & & & & $-.0525(-6.49)$ & \\
\hline \multicolumn{7}{|l|}{19} \\
\hline \multirow{2}{*}{\multicolumn{7}{|c|}{$\frac{20 a}{20 b}$}} \\
\hline & & & & & & \\
\hline \multicolumn{7}{|c|}{$-.0393(-6.33)$} \\
\hline 22 & & & & $1.38(3.29)$ & $-.0169(-5.86)$ & \\
\hline \multicolumn{6}{|c|}{$4.18(4.23)$} & $1.05(2.24)$ \\
\hline \multicolumn{7}{|c|}{$1.56(2.01)$} \\
\hline 24 & & & & & $-.0400(-13.1)$ & \\
\hline \multicolumn{7}{|l|}{25} \\
\hline \multicolumn{7}{|r|}{$1.08(2.19)$} \\
\hline \multicolumn{7}{|c|}{$\begin{array}{r}-.0195(-3.99) \\
\end{array}$} \\
\hline \multicolumn{7}{|c|}{$[-.0149(-3.42)$} \\
\hline \\
\hline $29 b$ & & & & & & \\
\hline 30 & & & & & $-.0481(-5.01)$ & \\
\hline
\end{tabular}

"nopass" - "traffic control device for the vehicle at fault is a «no passing zone»" indicator variable

"Incontr" - "traffic control device for the vehicle at fault is a «lane control»" indicator variable

"fl" _ - "traffic control device for the vehicle at fault is flashing signal" indicator

" $\mathrm{X}_{33}$ " _ - "at least one of the vehicles involved was on fire" indicator variable

"X $\mathrm{X}_{34}$ _ - "age (in years) of the driver at fault" quantitative variable 
Table B.4 (Continued)

\begin{tabular}{|c|c|c|c|c|c|c|}
\hline \multirow{2}{*}{ \# } & \multicolumn{6}{|c|}{ Coefficient (t-ratio) } \\
\hline & $x_{35}$ & $\begin{array}{c}\operatorname{maxpass} \\
{\left[\mathrm{X}_{27}\right]}\end{array}$ & $\begin{array}{c}\text { youngdrv } \\
{\left[\mathrm{X}_{34}\right]}\end{array}$ & olddrv $\left[X_{34}\right]$ & $\mathrm{mm}\left[\mathrm{X}_{35}\right]$ & \\
\hline $1^{\text {**}}$ & & $-.383(-3.91)$ & & & & \\
\hline $2 a$ & & & & & & \\
\hline $2 b$ & & & & $-1.32(-3.34)$ & & \\
\hline 3 & $-.305(-2.74)$ & & & & & \\
\hline 4 & & & & & & \\
\hline 5 & $2.29(2.13)$ & & & & & \\
\hline 6 & & & & & & \\
\hline $7 a$ & & $.496(2.78)$ & & & & \\
\hline $7 b$ & $.818(2.46)$ & & & & & \\
\hline $7 c$ & & & & & & \\
\hline 8 & & & & & & \\
\hline 9 & & & & & & \\
\hline 10 & & $.189(2.82)$ & & & & \\
\hline $11 \mathrm{a}$ & & & & & & \\
\hline $11 b$ & & & & & & \\
\hline 12 & & & & & & \\
\hline 13 & & & $-.0186(-2.88)$ & & & \\
\hline 14 & & & & & & \\
\hline 15 & & & & & & \\
\hline 16 & & & & & $.457(3.14)$ & \\
\hline 17 & & & & & & \\
\hline 18 & & & & & & \\
\hline 19 & & & & & & \\
\hline $20 a$ & & & & & & \\
\hline $20 b$ & & & & & & \\
\hline 21 & $-.322(-1.97)$ & & & & & \\
\hline$\overline{22}$ & & & & & $.254(2.72)$ & \\
\hline $23 a$ & & & & & & \\
\hline $23 b$ & $2.66(3.08)$ & & & & & \\
\hline 24 & $-.456(-5.71)$ & & & & & \\
\hline 25 & & & $-.0218(-3.14)$ & & & \\
\hline 26 & & & & & & \\
\hline 27 & & & & & & \\
\hline 28 & & & & & $.493(3.33)$ & \\
\hline $29 a$ & & & & & & \\
\hline $29 \mathrm{~b}$ & & & & & & \\
\hline 30 & & & & & & \\
\hline
\end{tabular}

... - positive coefficient … - negative coefficient.

"X ${ }_{35}$ " - "gender of the driver at fault: 1 - female, 0 - male" indicator variable

"maxpass" - "the largest number of occupants in all vehicles involved" indicator variable

"youngdrv" - "the driver at fault is younger than the other driver involved" indicator variable

"olddrv" - "the driver at fault is older than the other driver involved" indicator var.

"mm" - - "two male drivers involved into a two-vehicle accident" indicator var. 
Table B.5 Tests of car-SUV separation in 2004 accident causation study ${ }^{21}$

\begin{tabular}{|c|c|c|c|c|c|c|c|c|c|c|}
\hline \# & & & Model name & $M$ & K & $L L\left(\boldsymbol{\beta}_{\mathbf{m}}\right)$ & $\sum L L\left(\boldsymbol{\beta}_{\mathbf{m}}\right)$ & df & $p$-value & conclusion* \\
\hline \multirow{2}{*}{2} & \multirow{2}{*}{\multicolumn{2}{|c|}{ 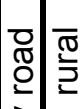 }} & (car/SUV)+(car/SUV) & 3 & 7 & -1426.61 & -1418.48 & 14 & 0.30 & Car $=$ SUV \\
\hline & & & (car/SUV)+(truck) & 2 & 2 & -87.04 & -86.24 & 2 & 0.45 & Car $=$ SUV \\
\hline \multirow{2}{*}{$\begin{array}{l}4 \\
5\end{array}$} & \multirow{2}{*}{ 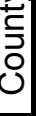 } & \multirow{2}{*}{$\begin{array}{l}\frac{c}{\sqrt{\sigma}} \\
\frac{0}{5} \\
\frac{0}{J}\end{array}$} & $(\mathrm{car} / \mathrm{SUV})+(\mathrm{car} / \mathrm{SUV})$ & 3 & 6 & -246.94 & -240.33 & 12 & 0.35 & Car $=$ SUV \\
\hline & & & (car/SUV)+(truck) & 2 & 5 & -16.14 & -15.11 & 5 & 0.84 & Car $=$ SUV \\
\hline \multirow{2}{*}{$\frac{7}{8}$} & \multirow[b]{2}{*}{$\stackrel{0}{\pi}$} & $\bar{\pi}$ & (car/SUV)+(car/SUV) & 3 & 7 & -327.44 & -317.43 & 14 & 0.13 & Car $=$ SUV \\
\hline & & 구 & (car/SUV)+(truck) & 2 & 6 & -128.79 & -127.07 & 6 & 0.75 & Car $=$ SUV \\
\hline \multirow{2}{*}{$\frac{10}{11}$} & \multirow{2}{*}{ 离 } & $\frac{c}{\sigma}$ & (car/SUV)+(car/SUV) & 3 & 7 & -339.99 & -333.09 & 14 & 0.46 & Car $=$ SUV \\
\hline & & 吕 & (car/SUV)+(truck) & 2 & 7 & -203.66 & -201.12 & 7 & 0.65 & Car $=$ SUV \\
\hline \multirow{2}{*}{$\begin{array}{l}13 \\
14\end{array}$} & \multirow{2}{*}{$\begin{array}{l}\frac{\Phi}{5} \\
\\
0\end{array}$} & & (car/SUV)+(car/SUV) & 3 & 7 & -537.67 & -530.30 & 14 & 0.40 & Car $=$ SUV \\
\hline & & 2 & (car/SUV)+(truck) & 2 & 6 & -128.69 & -123.18 & 6 & 0.88 & Car $=$ SUV \\
\hline \multirow{2}{*}{$\begin{array}{l}16 \\
17 \\
\end{array}$} & \multirow{2}{*}{$\begin{array}{l}\frac{0}{ \pm} \\
\frac{\pi}{\omega} \\
\end{array}$} & $\frac{c}{\varpi}$ & $(\mathrm{car} / \mathrm{SUV})+(\mathrm{car} / \mathrm{SUV})$ & 3 & 7 & -716.67 & -707.19 & 14 & 0.17 & Car $=$ SUV \\
\hline & & 旁 & (car/SUV)+(truck) & 2 & 4 & -57.96 & -57.47 & 4 & 0.91 & Car $=$ SUV \\
\hline \multirow{4}{*}{$\begin{array}{l}\frac{19}{20} \\
22 \\
23\end{array}$} & \multirow{4}{*}{ 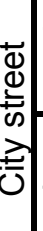 } & $\bar{\sigma}$ & (car/SUV)+(car/SUV) & 3 & 11 & -525.56 & -519.80 & 22 & 0.97 & Car $=$ SUV \\
\hline & & $\overline{2}$ & (car/SUV)+(truck) & 2 & 2 & -35.71 & -35.33 & 2 & 0.68 & Car $=$ SUV \\
\hline & & $\frac{1}{\pi}$ & $(\mathrm{car} / \mathrm{SUV})+(\mathrm{car} / \mathrm{SUV})$ & 3 & 12 & -6259.14 & -6246.99 & 24 & 0.44 & Car $=$ SUV \\
\hline & & 峜 & (car/SUV)+(truck) & 2 & 7 & -323.90 & -322.02 & 7 & 0.81 & Car $=$ SUV \\
\hline \multirow{4}{*}{$\frac{25}{26}$} & \multirow{4}{*}{\multicolumn{2}{|c|}{ 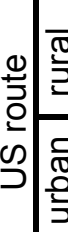 }} & (car/SUV)+(car/SUV) & 3 & 7 & -404.95 & -399.41 & 14 & 0.68 & Car $=$ SUV \\
\hline & & & (car/-SUV)+(truck) & 2 & 5 & -147.16 & -143.83 & 5 & 0.25 & Car $=$ SUV \\
\hline & & & $(\mathrm{car} / \mathrm{SUV})+(\mathrm{car} / \mathrm{SUV})$ & 3 & 11 & -1088.61 & -1081.30 & 22 & 0.88 & Car $=$ SUV \\
\hline & & & (car/SUV)+(truck) & 2 & 5 & -117.72 & -115.99 & 5 & 0.63 & Car $=$ SUV \\
\hline
\end{tabular}

* For all models 1-29 we find that "Car = SUV", which means that in 2004 unsafespeed-related accident causation study cars and SUVs can be considered together.

${ }^{21}$ These tests are intended for testing whether in two-vehicle accidents cars and SUVs can be considered together or must be considered separately. The testing is done for all two-vehicle accident best final models by using the likelihood ratio test given in Equation (2.5). Please refer to Equation (2.5) for explanation of the quantities reported in the table. The $p$-values given in the next to last column are the probability values of the test statistic under the zero hypothesis (which is that cars and SUVs can be considered together). 
Table B.6 Tests of car-SUV separation in 2006 accident causation study

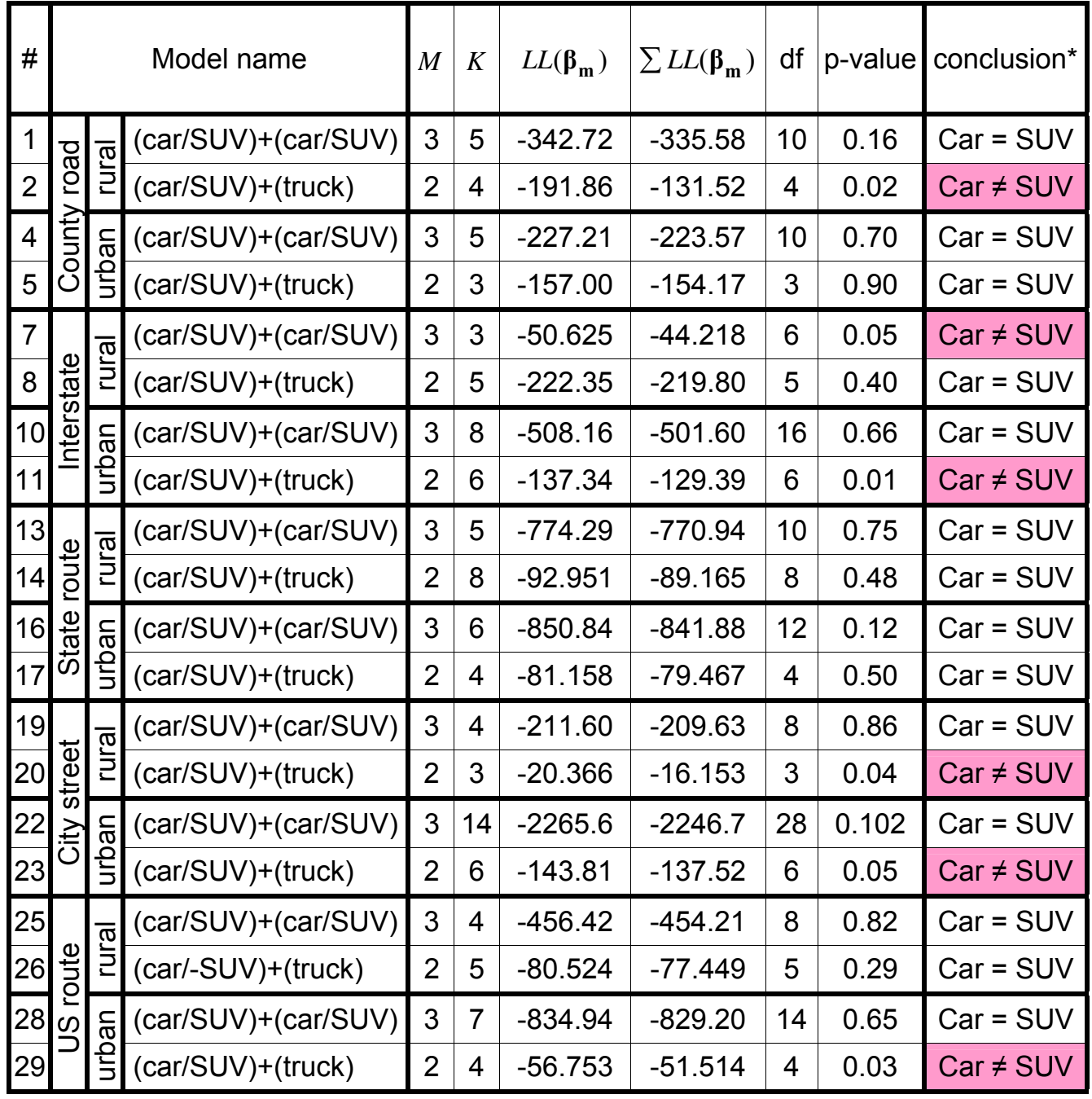

* For all models 2, 7, 11, 20, 23 and 29 we find that "Car $\neq$ SUV", which means that for these models cars and SUVs must be considered separately in 2006 accident causation study. For all other models we find that "Car = SUV", which means that cars and SUVs can be considered together. 
Appendix C.

Table C.1 Road classes and accident types in 2004 accident severity study

\begin{tabular}{|c|c|c|c|c|c|c|c|c|}
\hline \multirow{3}{*}{$\#$} & \multirow{3}{*}{\multicolumn{3}{|c|}{$\begin{array}{c}\text { Road-class-accident-type } \\
\text { combination }\end{array}$}} & \multicolumn{5}{|c|}{ Number of observations } \\
\hline & & & & \multirow{2}{*}{ all } & \multicolumn{4}{|c|}{ available for the models* } \\
\hline & & & & & total & fatal & injury & PDO \\
\hline 1 & \multirow{6}{*}{ 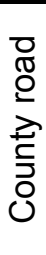 } & \multirow{3}{*}{ 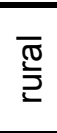 } & $\left(\mathrm{car} / \mathrm{SUV}^{* *}\right)+(\mathrm{car} / \mathrm{SUV})$ & 7260 & 2788 & 22 & 741 & 2025 \\
\hline 2 & & & $($ car/SUV)+(truck***) & 649 & 615 & 5 & 143 & 467 \\
\hline 3 & & & one vehicle & 18121 & 9433 & 115 & 2289 & 7029 \\
\hline 4 & & \multirow{3}{*}{ 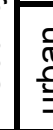 } & $(\mathrm{car} / \mathrm{SUV})+(\mathrm{car} / \mathrm{SUV})$ & 1861 & 1400 & 1 & 293 & 1106 \\
\hline 5 & & & (car/SUV)+(truck) & 143 & 120 & 0 & 21 & 99 \\
\hline 6 & & & one vehicle & 980 & 713 & 3 & 165 & 545 \\
\hline 7 & \multirow{7}{*}{ 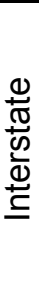 } & \multirow{4}{*}{ 휼 } & $($ car/SUV)+(car/SUV) & 1044 & 955 & $\overline{3}$ & 157 & 795 \\
\hline $8 a$ & & & (car)+(truck) & 516 & 470 & 6 & 78 & 386 \\
\hline $8 \mathrm{~b}$ & & & (SUV)+(truck) & 295 & 244 & 1 & 58 & 185 \\
\hline 9 & & & one vehicle & 3351 & 3284 & 23 & 510 & 2751 \\
\hline 10 & & \multirow{3}{*}{$\begin{array}{l}\frac{}{\mathbb{ত}} \\
\frac{0}{J}\end{array}$} & $(\mathrm{car} / \mathrm{SUV})+(\mathrm{car} / \mathrm{SUV})$ & 2234 & 1698 & 4 & 258 & 1436 \\
\hline 11 & & & (car/SUV)+(truck) & 1085 & 756 & 5 & 122 & 629 \\
\hline 12 & & & one vehicle & 1614 & 1526 & 14 & 345 & 1167 \\
\hline 13 & \multirow{8}{*}{ 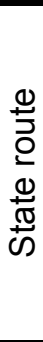 } & \multirow{3}{*}{$\overline{\widetilde{\alpha}}$} & $($ car/SUV $)+($ car/SUV $)$ & 4788 & 1979 & 23 & 638 & 1318 \\
\hline 14 & & & (car/SUV)+(truck) & 683 & 653 & 22 & 195 & 436 \\
\hline 15 & & & one vehicle & 9798 & 6368 & 50 & 1200 & 5118 \\
\hline $16 a$ & & \multirow{5}{*}{$\begin{array}{l}\frac{c}{\mathbb{0}} \\
\text { 인 }\end{array}$} & (car)+(car) & 2701 & 2318 & 2 & 581 & 1735 \\
\hline $16 b$ & & & (car)+(SUV) & 3872 & 3344 & 4 & 783 & 2557 \\
\hline $16 \mathrm{c}$ & & & $($ SUV)+(SUV) & 1473 & 1084 & 6 & 231 & 847 \\
\hline 17 & & & (car/SUV)+(truck) & 641 & 339 & 2 & 69 & 268 \\
\hline 18 & & & one vehicle & 1502 & 1381 & 9 & 308 & 1064 \\
\hline 19 & \multirow{7}{*}{ 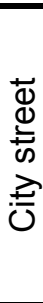 } & \multirow{3}{*}{$\stackrel{\bar{\pi}}{\underline{2}}$} & (car/SUV)+(car/SUV) & 3820 & 2610 & 4 & 591 & 2015 \\
\hline 20 & & & (car/SUV)+(truck) & 263 & 175 & 1 & 35 & 739 \\
\hline 21 & & & one vehicle & 2412 & 2033 & 13 & 495 & 1525 \\
\hline 22 & & \multirow{4}{*}{ 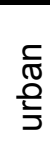 } & (car/SUV)+(car/SUV) & 63367 & 33141 & 28 & 7290 & 25823 \\
\hline $23 a$ & & & (car)+(truck) & 2321 & 1421 & 3 & 236 & 1182 \\
\hline $23 b$ & & & (SUV)+(truck) & 1273 & 990 & 3 & 145 & 842 \\
\hline 24 & & & one vehicle & 12549 & 6431 & 64 & 2249 & 4118 \\
\hline 25 & \multirow{6}{*}{$\begin{array}{l}\stackrel{\Phi}{J} \\
\stackrel{0}{0} \\
\stackrel{\omega}{J}\end{array}$} & \multirow{3}{*}{$\begin{array}{l}\bar{\pi} \\
\stackrel{\sqrt[T]{2}}{2}\end{array}$} & (car/SUV)+(car/SUV) & 2592 & 1499 & 17 & 484 & 998 \\
\hline 26 & & & (car/SUV)+(truck) & 566 & 413 & 21 & 154 & 238 \\
\hline 27 & & & one vehicle & 4211 & 2982 & 24 & 508 & 2450 \\
\hline 28 & & \multirow{3}{*}{$\begin{array}{l}\frac{c}{\sqrt{0}} \\
\frac{0}{J} \\
\frac{0}{J}\end{array}$} & $($ car/SUV)+(car/SUV) & 6931 & 4839 & 6 & 1219 & 3614 \\
\hline 29 & & & (car/SUV)+(truck) & 752 & 704 & 3 & 163 & 538 \\
\hline 30 & & & one vehicle & 1073 & 1063 & 13 & 292 & 758 \\
\hline
\end{tabular}

* - observations available for the best final estimated statistical models after exclusion of all missing observations

** - "SUV" includes sport utility vehicles, pickups and vans

*** - "truck" includes any possible kind of truck or tractor 
Table C.2 Road classes and accident types in 2006 accident severity study

\begin{tabular}{|c|c|c|c|c|c|c|c|c|}
\hline \multirow{3}{*}{ \# } & \multirow{3}{*}{\multicolumn{3}{|c|}{$\begin{array}{c}\text { Road-class-accident-type } \\
\text { combination }\end{array}$}} & \multicolumn{5}{|c|}{ Number of observations } \\
\hline & & & & \multirow{2}{*}{ all } & \multicolumn{4}{|c|}{ available for the models* } \\
\hline & & & & & total & fatal & injury & PDO \\
\hline 1 & \multirow{8}{*}{ 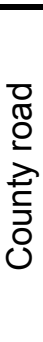 } & & $\left(\right.$ car/SUV $\left.{ }^{* *}\right)+($ car/SUV $)$ & 5966 & 4323 & 14 & 369 & 3940 \\
\hline 2 & & & $(\mathrm{car} / \mathrm{SUV})+\left(\right.$ truck $\left.^{* * *}\right)$ & 345 & 286 & 3 & 42 & 241 \\
\hline 3 & & & one vehicle & 16165 & 14733 & 143 & 3313 & 11277 \\
\hline $4 a$ & & \multirow{5}{*}{ 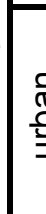 } & (car)+(car) & 536 & 489 & 0 & 107 & 275 \\
\hline $4 \mathrm{~b}$ & & & (car)+(SUV) & 691 & 232 & 0 & 40 & 192 \\
\hline $4 c$ & & & (SUV)+(SUV) & 261 & 225 & 0 & 37 & 188 \\
\hline 5 & & & (car/SUV)+(truck) & 80 & 80 & 1 & 5 & 74 \\
\hline 6 & & & one vehicle & 800 & 745 & 2 & 174 & 569 \\
\hline 7 & \multirow{6}{*}{ 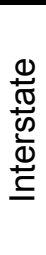 } & \multirow{3}{*}{$\begin{array}{l}\overline{\widetilde{\pi}} \\
\frac{2}{2}\end{array}$} & $($ car/SUV)+(car/SUV) & 1124 & 994 & 6 & 167 & 821 \\
\hline 8 & & & (car/SUV)+(truck) & 758 & 649 & 11 & 79 & 559 \\
\hline 9 & & & one vehicle & 3736 & 3676 & 22 & 571 & 3083 \\
\hline 10 & & \multirow{3}{*}{$\begin{array}{l}\text { 党 } \\
\text { 光 }\end{array}$} & (car/SUV)+(car/SUV) & 2395 & 2178 & 1 & 303 & 1874 \\
\hline 11 & & & (car/SUV)+(truck) & 850 & 692 & 2 & 89 & 601 \\
\hline 12 & & & one vehicle & 1884 & 1834 & 13 & 397 & 1424 \\
\hline 13 & \multirow{6}{*}{ 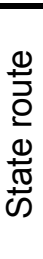 } & \multirow{3}{*}{$\frac{\bar{\pi}}{2}$} & (car/SUV)+(car/SUV) & 4582 & 763 & 28 & 274 & 461 \\
\hline 14 & & & (car/SUV)+(truck) & 524 & 125 & 10 & 18 & 97 \\
\hline 15 & & & one vehicle & 10172 & 9611 & 81 & 1659 & 7871 \\
\hline 16 & & \multirow{3}{*}{$\begin{array}{l}\frac{1}{\pi} \\
\frac{0}{5} \\
\text { 이 }\end{array}$} & (car/SUV)+(car/SUV) & 7483 & 6224 & 7 & 1398 & 4819 \\
\hline 17 & & & (car/SUV)+(truck) & 510 & 482 & 2 & 65 & 415 \\
\hline 18 & & & one vehicle & 1715 & 1579 & 16 & 404 & 1159 \\
\hline 19 & \multirow{8}{*}{ 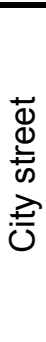 } & \multirow{3}{*}{ 㞼 } & $(\mathrm{car} / \mathrm{SUV})+(\mathrm{car} / \mathrm{SUV})$ & 2926 & 2103 & 1 & 473 & 1629 \\
\hline 20 & & & (car/SUV)+(truck) & 155 & 84 & 0 & 9 & 75 \\
\hline 21 & & & one vehicle & 2115 & 624 & 4 & 164 & 456 \\
\hline $22 a$ & & \multirow{5}{*}{$\begin{array}{l}\text { त } \\
\stackrel{c}{\varrho}\end{array}$} & (car)+(car) & 20109 & 13499 & 2 & 2983 & 10514 \\
\hline $22 \mathrm{~b}$ & & & (car)+(SUV) & 23000 & 8282 & 8 & 1866 & 6408 \\
\hline 22c & & & $($ SUV)+(SUV) & 7216 & 2544 & 3 & 601 & 1940 \\
\hline 23 & & & (car/SUV)+(truck) & 2323 & 1570 & 2 & 175 & 1393 \\
\hline 24 & & & one vehicle & 10869 & 4152 & 40 & 1561 & 2551 \\
\hline 25 & \multirow{6}{*}{$\begin{array}{l}\stackrel{\Phi}{ \pm} \\
\stackrel{0}{0} \\
\stackrel{\omega}{د}\end{array}$} & \multirow{3}{*}{$\overline{\frac{\pi}{2}}$} & $($ (car/SUV)+(car/SUV) & 2481 & 541 & 16 & 185 & 340 \\
\hline 26 & & & (car/SUV)+(truck) & 566 & 386 & 9 & 108 & 269 \\
\hline 27 & & & one vehicle & 4257 & 4019 & 26 & 642 & 3351 \\
\hline 28 & & \multirow{3}{*}{$\begin{array}{l}\text { 동 } \\
\text { 잏 }\end{array}$} & (car/SUV)+(car/SUV) & 6941 & 1680 & 2 & 463 & 1215 \\
\hline 29 & & & (car/SUV)+(truck) & 572 & 467 & 5 & 62 & 400 \\
\hline 30 & & & one vehicle & 1211 & 285 & 4 & 98 & 183 \\
\hline
\end{tabular}

* - observations available for the best final estimated statistical models after exclusion of all missing observations

** - "SUV" includes sport utility vehicles, pickups and vans

*** - "truck" includes any possible kind of truck or tractor 
Table C. 3 Speed limit data bins chosen in 2004 accident severity study

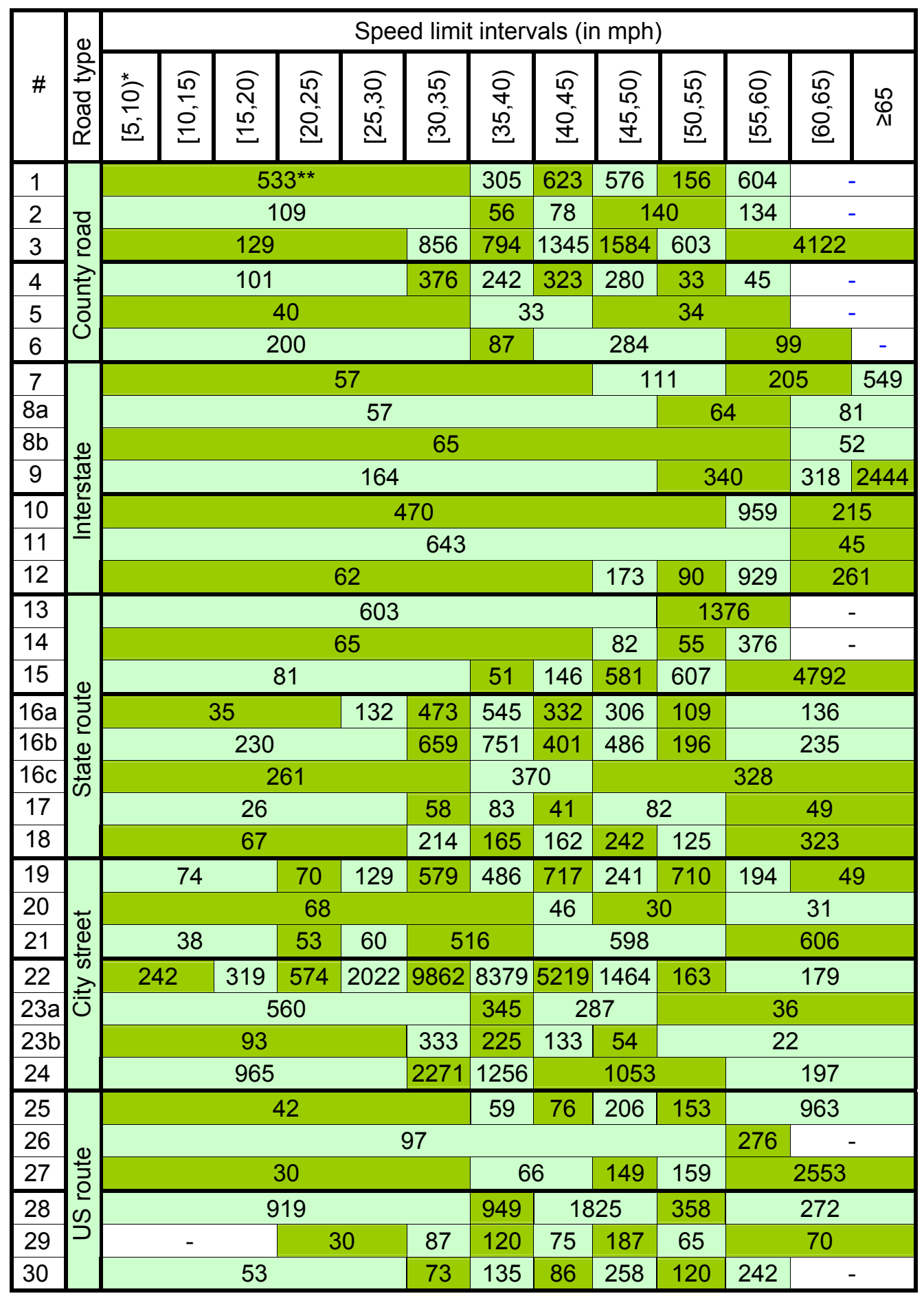

* - Interval $[5,10)$ includes speed limits larger or equal to $5 \mathrm{mph}$ and smaller than $10 \mathrm{mph}$. All other intervals are similarly defined.

** - Numbers printed on top the speed limit data bins inside the table, give data sample sizes in the corresponding bins. 
Table C.4 Speed limit data bins chosen in 2006 accident severity study

\begin{tabular}{|c|c|c|c|c|c|c|c|c|c|c|c|c|c|c|c|c|}
\hline \multirow[b]{2}{*}{ \# } & \multirow[b]{2}{*}{ 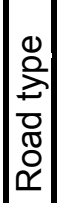 } & \multicolumn{15}{|c|}{ Speed limit intervals (in mph) } \\
\hline & & $\frac{\stackrel{*}{O}}{\sqrt{2}}$ & $\begin{array}{l}\frac{10}{2} \\
\stackrel{0}{\Xi}\end{array}$ & 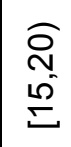 & $\begin{array}{l}\widehat{N} \\
\stackrel{N}{0} \\
\stackrel{N}{N}\end{array}$ & 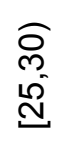 & $\begin{array}{l}\widehat{1} \\
\text { m } \\
\text { on } \\
\text { m }\end{array}$ & $\begin{array}{l}\widehat{o} \\
\text { to } \\
\text { p. } \\
\text { n. }\end{array}$ & \begin{tabular}{l}
$\widehat{b}$ \\
\multirow{8}{*}{} \\
d
\end{tabular} & $\begin{array}{l}\widehat{\circ} \\
0 \\
0 \\
0 \\
0\end{array}$ & $\begin{array}{l}\widehat{10} \\
0 \\
0 \\
0 \\
0\end{array}$ & $\begin{array}{l}\widehat{8} \\
6 \\
10 \\
0\end{array}$ & $\begin{array}{l}18 \\
0 \\
8 \\
8 \\
\mathbb{0}\end{array}$ & $\begin{array}{l}\widehat{a} \\
i 0^{0} \\
0\end{array}$ & $\begin{array}{l}\widehat{5} \\
\stackrel{0}{0} \\
\stackrel{0}{0}\end{array}$ & $\stackrel{2}{\stackrel{2}{\Lambda}}$ \\
\hline 1 & \multirow{8}{*}{ 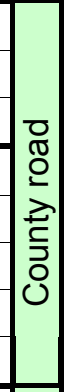 } & \multicolumn{8}{|c|}{$807^{\star *}$} & 345 & 98 & \multicolumn{2}{|c|}{223} & \multicolumn{3}{|c|}{ - } \\
\hline 2 & & \multirow{2}{*}{\multicolumn{6}{|c|}{$\begin{array}{c}45 \\
1127\end{array}$}} & 35 & 54 & \multicolumn{2}{|c|}{70} & 82 & & \multicolumn{3}{|c|}{ - } \\
\hline 3 & & & & & & & & \multicolumn{2}{|c|}{3233} & 2993 & 1021 & \multicolumn{4}{|c|}{6359} & - \\
\hline $4 a$ & & \multicolumn{6}{|c|}{26} & 72 & 110 & 111 & 9 & 11 & & \multicolumn{3}{|c|}{-} \\
\hline $4 b$ & & \multicolumn{6}{|c|}{84} & 51 & 44 & \multicolumn{4}{|c|}{53} & \multicolumn{3}{|c|}{-} \\
\hline $4 c$ & & \multicolumn{6}{|c|}{63} & 43 & 47 & \multicolumn{3}{|c|}{47} & \multicolumn{4}{|c|}{-} \\
\hline 5 & & \multicolumn{6}{|c|}{21} & \multicolumn{2}{|c|}{25} & & 16 & & & - & - & \\
\hline 6 & & & & 44 & & & 143 & 128 & 108 & & 295 & & & & - & \\
\hline 7 & & & & & & & 73 & & & & & 22 & & 177 & 336 & - \\
\hline 8 & & & & & & & 6 & & & & & & 2 & & & - \\
\hline 9 & $\frac{\Phi}{\sigma}$ & & & & & & & & & 7 & 4 & 43 & & 1019 & 2054 & - \\
\hline 10 & $\frac{\omega}{\omega}$ & & & 53 & & & 50 & 74 & 93 & 146 & 206 & 11 & & 33 & 31 & - \\
\hline 11 & I & & & & & & 21 & & & & & & 5. & & & - \\
\hline 12 & & & & & & & & & & 20 & 77 & 1131 & 2 & & 119 & - \\
\hline 13 & & & & & & 252 & & & & & & 5 & & & & - \\
\hline 14 & (1) & & & & & & 7 & & & & & & 68 & & & - \\
\hline 15 & 苛 & & & & & & & & & 935 & 748 & 7267 & & 296 & & - \\
\hline 16 & 0 & & & 47 & & 339 & 1470 & 1508 & 970 & 981 & 367 & & 442 & & & - \\
\hline 17 & $\frac{\pi}{0}$ & & & & 212 & & & & 580 & 71 & 46 & & 45 & & & - \\
\hline 18 & & & & & 77 & & & 205 & 172 & 282 & 143 & & 368 & & & - \\
\hline 19 & & & & 40 & & 100 & 523 & 438 & 577 & 208 & & & 117 & & & - \\
\hline 20 & & & & & 1665 & & & & 660 & & 381 & & & & - & \\
\hline 21 & $\mathbb{\Phi}$ & & & & & & & & & & & 2 & & & & - \\
\hline $22 a$ & ڤे & & & 50 & & 985 & 5440 & 3629 & 1928 & & 667 & & & & - & \\
\hline $22 b$ & $1 \geq$ & & & 35 & & 572 & 2901 & 2313 & 1620 & & & 541 & & & & - \\
\hline $22 c$ & & & & & 11 & & & & & & 1333 & & & & & - \\
\hline 23 & & & & 6 & & 134 & 561 & 442 & 227 & & & 120 & & & & - \\
\hline 24 & & & & 593 & & & 1598 & 818 & & & & 723 & & & & - \\
\hline 25 & & & & & & 8 & & & & 12 & 11 & 244 & 3 & & & - \\
\hline 26 & 0 & & & & & 84 & & & & & 24 & 40 & 6 & & & - \\
\hline 27 & సँّ & & & & & & 3134 & & & & & & & 869 & & - \\
\hline 28 & $\omega$ & & & & & 13 & & & & & & 737 & & & & - \\
\hline 29 & 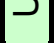 & & & & & 391 & & & & & & 7 & & & & - \\
\hline 30 & & & & & & 0 & & & & 11 & 6 & & 61 & & & - \\
\hline
\end{tabular}

* - Interval $[5,10)$ includes speed limits larger or equal to $5 \mathrm{mph}$ and smaller than $10 \mathrm{mph}$. All other intervals are similarly defined.

** - Numbers printed on top the speed limit data bins inside the table, give data sample sizes in the corresponding bins. 
Table C.5 Multinomial logit models for 2004 accident severity $^{22}$

\begin{tabular}{|c|c|c|c|c|c|c|c|c|}
\hline \multirow{3}{*}{ \# } & \multirow{3}{*}{\multicolumn{3}{|c|}{ Model name }} & \multirow{2}{*}{\multicolumn{2}{|c|}{ Log-likelihood }} & \multirow{3}{*}{$R^{2}$} & \multirow{2}{*}{\multicolumn{2}{|c|}{$\frac{\text { Coefficient (t-ratio) }}{\mathrm{X}_{29}}$}} \\
\hline & & & & & & & & \\
\hline & & & & model & restricted* & & fatality $\left[\boldsymbol{\beta}_{1}\right]$ & injury $\left[\boldsymbol{\beta}_{2}\right]$ \\
\hline 1 & \multirow{3}{*}{\multicolumn{2}{|c|}{ 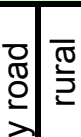 }} & (car/SUV)+(car/SUV) & -1636.3 & -1735.9 & .057 & $.108(3.61)$ & $.0255(5.15)$ \\
\hline 2 & & & (car/SUV)+(truck) & -250.47 & -287.90 & .130 & & $.0414(3.42)$ \\
\hline 3 & & & one vehicle & -5060.1 & -5816.0 & .130 & $.0382(3.47)$ & \\
\hline 4 & \multirow{3}{*}{\multicolumn{2}{|c|}{ כ) }} & (car/SUV)+(car/SUV) & -683.95 & -726.22 & .058 & $.0323(3.75)$ & $.0323(3.75)$ \\
\hline $5^{* *}$ & & & (car/SUV)+(truck) & -104.80 & -131.83 & .205 & & \\
\hline 6 & & & one vehicle & -359.79 & -404.07 & .110 & & \\
\hline 7 & \multirow{4}{*}{\multicolumn{2}{|c|}{ 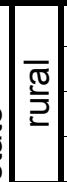 }} & (car/SUV)+(car/SUV) & -456.77 & -473.11 & .035 & & \\
\hline $8 a$ & & & (car)+(truck) & -198.37 & -219.34 & .095 & & \\
\hline $8 b$ & & & $($ SUV)+(truck) & -114.91 & -140.04 & .179 & & \\
\hline 9 & $\frac{\pi}{0}$ & & one vehicle & -1360.9 & -1550.4 & .122 & & \\
\hline 10 & 这 & \multirow{3}{*}{ 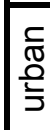 } & (car/SUV)+(car/SUV) & -710.09 & -751.00 & .054 & & \\
\hline 11 & $\subseteq$ & & (car/SUV)+(truck) & -336.87 & -363.30 & .073 & & \\
\hline 12 & & & one vehicle & -772.32 & -887.87 & .130 & & \\
\hline 13 & & \multirow{3}{*}{ 쥴 } & (car/SUV)+(car/SUV) & -1302.1 & -1360.4 & .043 & & $.0306(3.90)$ \\
\hline 14 & & & (car/SUV)+(truck) & -362.86 & -405.25 & .105 & & \\
\hline 15 & $\Phi$ & & one vehicle & -2188.0 & -2714.2 & .194 & & \\
\hline $16 a$ & 苛 & \multirow{5}{*}{\begin{tabular}{|l} 
\\
$\frac{1}{\widetilde{0}}$ \\
$\frac{0}{5}$ \\
$\frac{0}{5}$
\end{tabular}} & (car)+(car) & -1116.2 & -1157.9 & .036 & $.0340(5.14)$ & $.0340(5.14)$ \\
\hline $16 b$ & 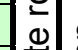 & & (car)+(SUV) & -1547.8 & -1608.4 & .038 & $.0225(4.36)$ & $.0225(4.36)$ \\
\hline $16 c$ & . & & (SUV)+(SUV) & -492.07 & -517.33 & .049 & $.0315(3.39)$ & $.0315(3.39)$ \\
\hline 17 & (s) & & (car/SUV)+(truck) & -173.40 & -183.09 & .053 & $.0418(2.89)$ & $.0418(2.89)$ \\
\hline 18 & & & one vehicle & -677.08 & -784.90 & .137 & & \\
\hline 19 & & \multirow{3}{*}{$\frac{\overline{0}}{\underline{2}}$} & $(\mathrm{car} / \mathrm{SUV})+(\mathrm{car} / \mathrm{SUV})$ & -1358.7 & -1425.1 & .047 & $.114(2.53)$ & $.0273(5.40)$ \\
\hline 20 & & & $($ car/SUV)+(truck) & -68.07 & -93.51 & .272 & & $.0676(3.17)$ \\
\hline 21 & + & & one vehicle & -1025.6 & -1203.4 & .148 & & \\
\hline 22 & 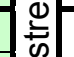 & \multirow{4}{*}{$\begin{array}{l} \\
\frac{1}{\sqrt{0}} \\
\frac{0}{\frac{0}{J}} \\
\end{array}$} & $($ car/SUV)+(car/SUV) & -14547 & -15236 & .045 & $.0938(2.76)$ & $.0304(11.8)$ \\
\hline $23 a$ & $\geqslant$ & & (car)+(truck) & -526.00 & -580.96 & .095 & $.0469(3.99)$ & $.0469(3.99)$ \\
\hline $23 b$ & ن. & & (SUV)+(truck) & -344.88 & -366.77 & .060 & $.0640(4.41)$ & $.0640(4.41)$ \\
\hline 24 & & & one vehicle & -4004.8 & -4493.6 & .109 & & \\
\hline 25 & & \multirow{3}{*}{ 휸 } & (car/SUV)+(car/SUV) & -978.44 & -1029.3 & .049 & $.340(2.48)$ & $.0409(4.54)$ \\
\hline 26 & & & (car/SUV)+(truck) & -270.09 & -309.90 & .128 & & $.0720(3.02)$ \\
\hline 27 & 站 & & one vehicle & -1251.3 & -1496.3 & .164 & & \\
\hline 28 & 힁 & \multirow{3}{*}{$\begin{array}{l}\frac{c}{\sqrt{d}} \\
\text { 은 } \\
\end{array}$} & (car/SUV)+(car/SUV) & -2345.1 & -2457.6 & .046 & $.0263(5.76)$ & $.0263(5.76)$ \\
\hline 29 & D. & & (car/SUV)+(truck) & -311.62 & -336.81 & .075 & $.0307(2.52)$ & $.0307(2.52)$ \\
\hline 30 & & & one vehicle & -549.94 & -639.11 & .140 & & \\
\hline \multicolumn{9}{|c|}{$\begin{array}{l}\ldots . . . \text { - positive coefficient } \\
\text { * } \\
\text { - restricted log-likelihood found by setting all coefficients except intercepts to zero } \\
\text { (with the exception of model } 5 \text {, in case of which intercepts are also set to zero) } \\
\text { - models are estimated by using procedure } A \text { on page } 32 \text {, except the models } \\
\text { marked by bold numbers and estimated by using procedure B on page } 33\end{array}$} \\
\hline
\end{tabular}

22 See Equation (2.4), where outcomes "1", "2", "3" correspond to "fatality", "injury", "PDO”. Only statistically significant coefficients, which are components of vectors $\boldsymbol{\beta}_{\mathbf{1}}$ and $\boldsymbol{\beta}_{\mathbf{2}}$, are given. 
Table C.5 (Continued)

\begin{tabular}{|c|c|c|c|c|c|c|}
\hline \multirow{3}{*}{ \# } & \multicolumn{6}{|c|}{ Coefficient (t-ratio) } \\
\hline & \multicolumn{2}{|c|}{ constant } & \multicolumn{2}{|c|}{ wint $\left[\mathrm{X}_{3}\right]$} & \multicolumn{2}{|c|}{$\operatorname{sum}\left[\mathrm{X}_{3}\right]$} \\
\hline & fatality & injury & fatality & injury & fatality & injury \\
\hline 1 & $-12.0(-6.27)$ & $-3.30(-12.4)$ & $-.260(-2.61)$ & $-.260(-2.61)$ & & \\
\hline 2 & $-7.41(-5.59)$ & $-4.07(-6.90)$ & & & & \\
\hline 3 & $-6.43(-10.7)$ & $-1.45(-13.7)$ & & & $.232(3.51)$ & $.232(3.51)$ \\
\hline 4 & $-9.13(-8.46)$ & $-3.57(-8.23)$ & & & & \\
\hline 5 & & $-2.78(-3.72)$ & & & & \\
\hline 6 & $-6.90(-9.19)$ & $-2.50(-9.49)$ & & & & \\
\hline 7 & $-6.25(-10.2)$ & $-2.33(-10.7)$ & & & & \\
\hline $8 a$ & $-5.97(-5.85)$ & $-1.87(-7.79)$ & & & & \\
\hline $8 b$ & $-6.76(-5.65)$ & $-3.35(-4.72)$ & & & & \\
\hline 9 & $-4.84(-20.4)$ & $-3.04(-12.7)$ & & & & \\
\hline 10 & $-8.01(-8.12)$ & $-2.40(-11.2)$ & & & & \\
\hline 11 & $-6.23(-10.0)$ & $-2.53(-10.4)$ & & & & \\
\hline 12 & $-5.88(-12.5)$ & $-3.25(-9.55)$ & & & $1.13(2.09)$ & \\
\hline 13 & $-5.24(-13.2)$ & $-3.47(-7.44)$ & & & & \\
\hline 14 & $-4.30(-9.91)$ & $-2.88(-7.34)$ & $-.619(-2.58)$ & $-.619(-2.58)$ & & \\
\hline 15 & $-4.31(-16.9)$ & $-1.64(-11.7)$ & & & & \\
\hline $16 \mathrm{a}$ & $-9.26(-8.86)$ & $-3.02(-3.09)$ & & & & \\
\hline $16 \mathrm{~b}$ & $-8.88(-11.6)$ & $-3.14(-10.3)$ & & & & \\
\hline $16 c$ & $-7.13(-8.91)$ & $-3.22(-7.13)$ & & & & $.412(2.37)$ \\
\hline 17 & $-7.42(-6.33)$ & $-3.22(-5.16)$ & & & & \\
\hline 18 & $-6.31(-10.7)$ & $-1.60(-11.3)$ & & & $1.96(2.76)$ & \\
\hline 19 & $-11.3(-5.04)$ & $-2.25(-8.63)$ & $-.276(-2.34)$ & $-.276(-2.34)$ & & \\
\hline 20 & $-6.22(-5.18)$ & $-5.50(-4.69)$ & & & & \\
\hline 21 & $-5.62(-17.1)$ & $-3.16(-14.6)$ & $-.317(-2.24)$ & $-.317(-2.24)$ & & \\
\hline 22 & $-14.0(-8.80)$ & $-2.93(-26.5)$ & & $-.0872(-2.45)$ & & \\
\hline $23 a$ & $-9.67(-8.74)$ & $-3.87(-8.47)$ & & & & \\
\hline $23 b$ & $-8.24(-9.27)$ & $-4.38(-7.74)$ & & & & \\
\hline 24 & $-7.38(-16.7)$ & $-4.30(-13.9)$ & $-1.09(-2.65)$ & $-.410(-5.95)$ & & \\
\hline 25 & $-22.6(-3.00)$ & $-3.11(-6.50)$ & $-.287(-2.12)$ & $-.287(-2.12)$ & & \\
\hline 26 & $-4.73(-6.79)$ & $-5.49(-4.06)$ & & & & \\
\hline 27 & $-5.56(-22.6)$ & $-1.86(-11.2)$ & & & & \\
\hline 28 & $-8.57(-15.6)$ & $-3.12(-9.77)$ & & & & \\
\hline 29 & $-8.67(-7.46)$ & $-4.01(-6.27)$ & & & & \\
\hline 30 & $-5.20(-10.1)$ & $-1.61(-9.51)$ & & & $.429(2.43)$ & $.429(2.43)$ \\
\hline $\begin{array}{l}\ldots . \\
\ldots .\end{array}$ & neative & $\begin{array}{l}\text { ficient } \\
\text { fficient }\end{array}$ & & & & \\
\hline
\end{tabular}

"constant" - "constant term (intercept)" quantitative variable

"wint" - - "winter season" indicator variable

"sum" _ - "summer season" indicator variable 
Table C.5 (Continued)

\begin{tabular}{|c|c|c|c|c|c|c|}
\hline \multirow{3}{*}{ \# } & \multicolumn{6}{|c|}{ Coefficient (t-ratio) } \\
\hline & \multicolumn{2}{|c|}{ fall $\left[X_{3}\right]$} & \multicolumn{2}{|c|}{$\operatorname{mon}\left[\mathrm{X}_{4}\right]$} & \multicolumn{2}{|c|}{ tues $\left[\mathrm{X}_{4}\right]$} \\
\hline & fatality & injury & fatality & injury & fatality & injury \\
\hline \multicolumn{7}{|l|}{1} \\
\hline 2 & & & $2.67(2.11)$ & $.933(3.29)$ & & \\
\hline 3 & & & & & & \\
\hline 4 & $-.445(-2.68)$ & $-.445(-2.68)$ & & & & \\
\hline \multirow{2}{*}{\multicolumn{7}{|c|}{5}} \\
\hline & & & & & & \\
\hline \multicolumn{7}{|l|}{7} \\
\hline \multicolumn{7}{|l|}{$8 a$} \\
\hline $8 b$ & & & & & & $1.10(2.57)$ \\
\hline 9 & & $-.264(-2.04)$ & & & & \\
\hline 10 & & & & & $-.457(-2.14)$ & $-.457(-2.14)$ \\
\hline \multicolumn{7}{|l|}{11} \\
\hline 12 & & & & & & \\
\hline \multicolumn{2}{|l|}{$\frac{15}{13}$} & & & & & \\
\hline \multicolumn{7}{|c|}{$.524(1.99)$} \\
\hline \multicolumn{7}{|l|}{15} \\
\hline \multicolumn{7}{|l|}{$16 a$} \\
\hline \multirow{2}{*}{\multicolumn{7}{|c|}{$\frac{16 \mathrm{~b}}{16 \mathrm{c}}$}} \\
\hline \multirow{2}{*}{\multicolumn{7}{|c|}{$.823(2.50)$}} \\
\hline & & & & & & \\
\hline \multicolumn{7}{|l|}{18} \\
\hline \multirow{2}{*}{\multicolumn{7}{|c|}{19}} \\
\hline & \multicolumn{6}{|c|}{20} \\
\hline 21 & & & $-.374(-2.09)$ & $-.374(-2.09)$ & & \\
\hline 22 & & & & & $1.03(2.23)$ & \\
\hline \multicolumn{7}{|c|}{ 23a } \\
\hline \multirow{2}{*}{\multicolumn{7}{|c|}{$23 b$}} \\
\hline & & & & & & \\
\hline \multicolumn{7}{|l|}{25} \\
\hline \multicolumn{4}{|c|}{$1.08(2.04)$} & & & \\
\hline \multicolumn{5}{|l|}{27} & & \\
\hline 29 & & $-.679(-2.54)$ & & & & \\
\hline 30 & & & & & & \\
\hline
\end{tabular}

- positive coefficient

- negative coefficient

"fall" _ - "fall season" indicator variable

"mon" - "Monday" indicator variable

"tues" - "Tuesday" indicator variable 
Table C.5 (Continued)

\begin{tabular}{|c|c|c|c|c|c|c|}
\hline \multirow{3}{*}{ \# } & \multicolumn{6}{|c|}{ Coefficient (t-ratio) } \\
\hline & \multicolumn{2}{|c|}{ sund $\left[\mathrm{X}_{4}\right]$} & \multicolumn{2}{|c|}{ sat $\left[\mathrm{X}_{4}\right]$} & \multicolumn{2}{|c|}{ wed $\left[\mathrm{X}_{4}\right]$} \\
\hline & fatality & injury & fatality & injury & fatality & injury \\
\hline \multirow{2}{*}{\multicolumn{7}{|c|}{$\frac{1}{2}$}} \\
\hline & & & & & & \\
\hline \multicolumn{7}{|l|}{3} \\
\hline \multicolumn{7}{|l|}{4} \\
\hline \multirow{2}{*}{\multicolumn{7}{|c|}{$\begin{array}{l}5 \\
6\end{array}$}} \\
\hline & & & & & & \\
\hline \multicolumn{7}{|l|}{7} \\
\hline \multicolumn{7}{|l|}{$8 a$} \\
\hline \multicolumn{7}{|l|}{$\frac{00}{9}$} \\
\hline \multicolumn{7}{|l|}{10} \\
\hline \multicolumn{7}{|l|}{11} \\
\hline \multicolumn{7}{|l|}{12} \\
\hline 13 & $1.20(2.50)$ & & & & & \\
\hline \multirow{2}{*}{\multicolumn{7}{|c|}{$\begin{array}{l}\mathbf{1 4} \\
15\end{array}$}} \\
\hline & & & & & & \\
\hline \multicolumn{7}{|l|}{$16 \mathrm{a}$} \\
\hline \multicolumn{7}{|l|}{$16 \mathrm{~b}$} \\
\hline \multirow{2}{*}{\multicolumn{7}{|c|}{$\frac{16 c}{17}$}} \\
\hline & & & & & & \\
\hline \multicolumn{7}{|l|}{18} \\
\hline \multicolumn{7}{|l|}{19} \\
\hline \multirow{2}{*}{\multicolumn{7}{|c|}{$2.08(2.64)$}} \\
\hline & & & & & & \\
\hline \\
\hline \multicolumn{7}{|l|}{ 23a } \\
\hline \multirow{2}{*}{\multicolumn{7}{|c|}{24}} \\
\hline & & & & & & \\
\hline \multicolumn{7}{|l|}{25} \\
\hline \multicolumn{7}{|l|}{26} \\
\hline 27 & & & & & $-.397(-2.21)$ & $-.397(-2.21)$ \\
\hline \multicolumn{7}{|l|}{28} \\
\hline 29 & & & & & & \\
\hline 30 & & & & & & \\
\hline
\end{tabular}

- positive coefficient

- negative coefficient

\begin{tabular}{ll}
\hline "sund" $\quad$ - "Sunday" indicator variable \\
"sat" & - "Saturday" indicator variable \\
"wed" $\quad$ - "Wednesday" indicator variable
\end{tabular} 
Table C.5 (Continued)

\begin{tabular}{|c|c|c|c|c|c|c|}
\hline \multirow{3}{*}{ \# } & \multicolumn{6}{|c|}{ Coefficient (t-ratio) } \\
\hline & \multicolumn{2}{|c|}{ thday $\left[\mathrm{X}_{4}\right]$} & \multicolumn{2}{|c|}{$\operatorname{nigh}\left[\mathrm{X}_{5}\right]$} & \multicolumn{2}{|c|}{$\operatorname{morn}\left[X_{5}\right]$} \\
\hline & fatality & injury & fatality & injury & fatality & injury \\
\hline \multicolumn{7}{|l|}{1} \\
\hline \multirow{2}{*}{\multicolumn{7}{|c|}{2}} \\
\hline 3 & & & & & & \\
\hline \multicolumn{7}{|l|}{$\frac{3}{4}$} \\
\hline \multicolumn{7}{|l|}{5} \\
\hline \multicolumn{7}{|l|}{6} \\
\hline \multirow{2}{*}{\multicolumn{7}{|c|}{$\begin{array}{r}7 \\
8 a\end{array}$}} \\
\hline & & & & & & \\
\hline \multicolumn{7}{|l|}{$8 b$} \\
\hline \multicolumn{7}{|l|}{9} \\
\hline 10 & & & & & $-.414(-2.33)$ & $-.414(-2.33)$ \\
\hline \multicolumn{7}{|l|}{11} \\
\hline 12 & $-.517(-2.56)$ & $-.517(-2.56)$ & & & & \\
\hline \multirow{2}{*}{\multicolumn{7}{|c|}{13}} \\
\hline \multirow{2}{*}{\multicolumn{7}{|c|}{$\frac{14}{15}$}} \\
\hline & & & & & & \\
\hline \multicolumn{7}{|l|}{$16 \mathrm{a}$} \\
\hline \multirow{2}{*}{\multicolumn{7}{|c|}{$\frac{16 b}{16 c}$}} \\
\hline \multirow{2}{*}{\multicolumn{7}{|c|}{$\begin{array}{r}10 c \\
17\end{array}$}} \\
\hline & & & & & & \\
\hline \multicolumn{7}{|l|}{18} \\
\hline \multirow{2}{*}{\multicolumn{7}{|c|}{$\begin{array}{l}19 \\
20\end{array}$}} \\
\hline & & & & & & \\
\hline \multicolumn{7}{|l|}{21} \\
\hline \multicolumn{7}{|l|}{22} \\
\hline $23 a$ & & & $3.30(2.30)$ & & & \\
\hline \multicolumn{7}{|l|}{ 23b } \\
\hline \multicolumn{7}{|l|}{24} \\
\hline \multirow{2}{*}{\multicolumn{7}{|c|}{25}} \\
\hline \multirow{2}{*}{\multicolumn{7}{|c|}{27}} \\
\hline & & & & & & \\
\hline 28 & & & & & & \\
\hline 29 & & & & & & \\
\hline 30 & $1.73(2.71)$ & & & & & \\
\hline
\end{tabular}

- positive coefficient

- negative coefficient

"thday" - "Thursday" indicator variable

"nigh" - "late night hours: 1:00 to 5:00" indicator variable ${ }^{23}$

"morn" - "morning hours: 5:00 to 9:00" indicator variable

${ }^{23}$ We use military 24-hour time everywhere in our research. 
Table C.5 (Continued)

\begin{tabular}{|c|c|c|c|c|c|c|}
\hline \multirow{3}{*}{ \# } & \multicolumn{6}{|c|}{ Coefficient (t-ratio) } \\
\hline & \multicolumn{2}{|c|}{ dayt $\left[\mathrm{X}_{5}\right]$} & \multicolumn{2}{|c|}{ nocons $\left[\mathrm{X}_{13}\right]$} & \multicolumn{2}{|c|}{ cons $\left[\mathrm{X}_{13}\right]$} \\
\hline & fatality & injury & fatality & injury & fatality & injury \\
\hline \multirow{2}{*}{\multicolumn{7}{|c|}{1}} \\
\hline \multirow{2}{*}{\multicolumn{7}{|c|}{$\frac{2}{3}$}} \\
\hline & & & & & & \\
\hline \multicolumn{7}{|l|}{4} \\
\hline \multicolumn{7}{|l|}{5} \\
\hline \multirow{2}{*}{\multicolumn{7}{|c|}{$\frac{6}{7}$}} \\
\hline & & & & & & \\
\hline \multicolumn{7}{|l|}{$8 a$} \\
\hline \multirow{2}{*}{\multicolumn{7}{|c|}{$\begin{array}{r}8 \boldsymbol{b} \\
9\end{array}$}} \\
\hline & & & & & & \\
\hline \multicolumn{7}{|l|}{10} \\
\hline \multicolumn{7}{|l|}{11} \\
\hline 12 & & & $.510(2.19)$ & $.510(2.19)$ & & \\
\hline \multicolumn{7}{|l|}{13} \\
\hline \multicolumn{7}{|c|}{14} \\
\hline \multicolumn{7}{|c|}{15} \\
\hline $16 \mathrm{a}$ & $-.228(-2.15)$ & $-.228(-2.15)$ & & & & \\
\hline \multicolumn{7}{|c|}{$16 \mathrm{~b}$} \\
\hline \multirow{2}{*}{\multicolumn{7}{|c|}{$2.58(2.09)$}} \\
\hline & & & & & & \\
\hline \multicolumn{7}{|c|}{$.324(2.21)$} \\
\hline \multicolumn{7}{|c|}{19} \\
\hline \multicolumn{7}{|c|}{20} \\
\hline \multicolumn{7}{|c|}{21} \\
\hline \multirow{2}{*}{\multicolumn{7}{|c|}{$\begin{array}{l}22 \\
23 a\end{array}$}} \\
\hline & & & & & & \\
\hline \multicolumn{7}{|c|}{$23 b$} \\
\hline 24 & & & $.672(2.73)$ & $.672(2.73)$ & & \\
\hline \multicolumn{7}{|c|}{25} \\
\hline \multicolumn{7}{|l|}{26} \\
\hline 27 & $.282(2.40)$ & $.282(2.40)$ & & & & \\
\hline $28 \quad \longrightarrow$ & & & & & & \\
\hline 29 & & & & & & \\
\hline 30 & & & & & & \\
\hline
\end{tabular}

- positive coefficient

- negative coefficient

"dayt" - "day hours: 9:00 to 17:00" indicator variable

"nocons" - "no construction at the accident location" indicator variable

"cons" - "construction at the accident location" indicator variable 
Table C.5 (Continued)

\begin{tabular}{|c|c|c|c|c|c|c|}
\hline \multirow{3}{*}{ \# } & \multicolumn{6}{|c|}{ Coefficient (t-ratio) } \\
\hline & \multicolumn{2}{|c|}{ light $\left[\mathrm{X}_{14}\right]$} & \multicolumn{2}{|c|}{ dark $\left[\mathrm{X}_{14}\right]$} & \multicolumn{2}{|c|}{ day $\left[\mathrm{X}_{14}\right]$} \\
\hline & fatality & injury & fatality & injury & fatality & injury \\
\hline \multicolumn{7}{|l|}{1} \\
\hline \multicolumn{7}{|l|}{2} \\
\hline 3 & & & & & & $.199(3.74)$ \\
\hline \multicolumn{7}{|l|}{4} \\
\hline \multicolumn{7}{|l|}{5} \\
\hline \multicolumn{7}{|l|}{6} \\
\hline 7 & & & $845(4.25)$ & $.845(4.25)$ & & \\
\hline \multicolumn{7}{|l|}{$8 a$} \\
\hline $8 b$ & & & $1.22(3.07)$ & $1.22(3.07)$ & & \\
\hline \multicolumn{7}{|l|}{9} \\
\hline \multicolumn{7}{|l|}{10} \\
\hline \multicolumn{7}{|l|}{11} \\
\hline \multicolumn{7}{|l|}{12} \\
\hline 13 & & & $1.06(2.35)$ & & & \\
\hline 14 & & & $1.24(2.44)$ & & & \\
\hline 15 & & $261(3.10)$ & & & & \\
\hline \multicolumn{7}{|l|}{$16 a$} \\
\hline \multicolumn{7}{|l|}{$16 \mathrm{~b}$} \\
\hline \multicolumn{7}{|l|}{$16 c$} \\
\hline \multicolumn{7}{|l|}{17} \\
\hline \multicolumn{7}{|l|}{18} \\
\hline 19 & & & & & & $-.297(-2.75)$ \\
\hline \multicolumn{7}{|l|}{20} \\
\hline \multicolumn{7}{|l|}{21} \\
\hline \multicolumn{7}{|l|}{22} \\
\hline \multicolumn{7}{|l|}{$23 a$} \\
\hline \multicolumn{7}{|l|}{$23 b$} \\
\hline 24 & & & & & $-.824(-3.15)$ & \\
\hline 25 & & & $1.29(2.55)$ & & & \\
\hline \multicolumn{7}{|l|}{26} \\
\hline \multicolumn{7}{|l|}{27} \\
\hline 28 & & & & & & \\
\hline 29 & & & & & & \\
\hline 30 & & & & & & $.642(4.04)$ \\
\hline
\end{tabular}

- positive coefficient

- negative coefficient

"light" _ - "daylight time OR street lights lit up during dark time" indicator variable

"dark" _ - "dark time with no street lights" indicator variable

"day" _ - "daylight time" indicator variable 
Table C.5 (Continued)

\begin{tabular}{|c|c|c|c|c|c|c|}
\hline \multirow{3}{*}{ \# } & \multicolumn{6}{|c|}{ Coefficient (t-ratio) } \\
\hline & \multicolumn{2}{|c|}{ dawn $\left[\mathrm{X}_{14}\right]$} & \multicolumn{2}{|c|}{ darklamp $\left[\mathrm{X}_{14}\right]$} & \multicolumn{2}{|c|}{ precip $\left[\mathrm{X}_{15}\right]$} \\
\hline & fatality & injury & fatality & injury & fatality & injury \\
\hline \multirow{2}{*}{\multicolumn{7}{|c|}{1}} \\
\hline \multirow{2}{*}{\multicolumn{7}{|c|}{$\frac{2}{3}$}} \\
\hline & & & & & & \\
\hline \multicolumn{7}{|l|}{4} \\
\hline 5 & & & & & & $1.46(2.39)$ \\
\hline \multicolumn{7}{|l|}{6} \\
\hline \multirow{2}{*}{\multicolumn{7}{|c|}{$\frac{7}{8 a}$}} \\
\hline & & & & & & \\
\hline \multicolumn{7}{|l|}{$8 b$} \\
\hline \multirow{2}{*}{\multicolumn{7}{|c|}{10}} \\
\hline & & & & & & \\
\hline 12 & & & & & $-1.02(-6.41)$ & $-1.02(-6.41)$ \\
\hline \multicolumn{7}{|r|}{ 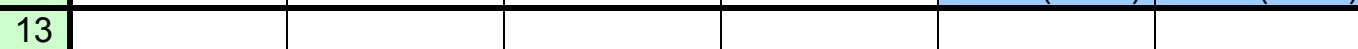 } \\
\hline \multirow{2}{*}{\multicolumn{7}{|c|}{$\begin{array}{l}\mathbf{1 4} \\
15\end{array}$}} \\
\hline 15 & & & & & & \\
\hline \multicolumn{7}{|l|}{$16 \mathrm{a}$} \\
\hline \multicolumn{7}{|l|}{$16 \mathrm{~b}$} \\
\hline \multirow{2}{*}{\multicolumn{7}{|c|}{$\frac{16 c}{17}$}} \\
\hline & & & & & & \\
\hline \multicolumn{7}{|l|}{18} \\
\hline \multicolumn{7}{|l|}{19} \\
\hline \multicolumn{7}{|c|}{$2.78(2.53)$} \\
\hline 21 & & & & & & \\
\hline $.188(4.76)$ & & & $.188(4.76)$ & $.188(4.76)$ & & \\
\hline \multicolumn{7}{|c|}{$.545(2.55)$} \\
\hline \multirow{2}{*}{\multicolumn{7}{|c|}{$.641(2.00)$}} \\
\hline & & & & & & \\
\hline \multicolumn{7}{|l|}{25} \\
\hline \multicolumn{7}{|l|}{26} \\
\hline 27 & & & & & & \\
\hline 29 & & & $.643(2.17)$ & $.643(2.17)$ & & \\
\hline 30 & & & & & & \\
\hline
\end{tabular}

- positive coefficient

- negative coefficient

"dawn" _ - "dawn OR dask" indicator variable

"darklamp" - "dark AND street lights on" indicator variable

"precip" - - "precipitation: rain OR snow OR sleet OR hail OR freezing rain" indicator variable 
Table C.5 (Continued)

\begin{tabular}{|c|c|c|c|c|c|c|}
\hline \multirow{3}{*}{ \# } & \multicolumn{6}{|c|}{ Coefficient (t-ratio) } \\
\hline & \multicolumn{2}{|c|}{ snow $\left[\mathrm{X}_{15}\right]$} & \multicolumn{2}{|c|}{ clear $\left[\mathrm{X}_{15}\right]$} & \multicolumn{2}{|c|}{ clo $\left[\mathrm{X}_{15}\right]$} \\
\hline & fatality & injury & fatality & injury & fatality & injury \\
\hline \multicolumn{7}{|l|}{1} \\
\hline \multicolumn{7}{|l|}{2} \\
\hline \multicolumn{7}{|l|}{3} \\
\hline \multicolumn{7}{|l|}{4} \\
\hline \multicolumn{7}{|l|}{5} \\
\hline \multicolumn{7}{|l|}{6} \\
\hline \multicolumn{7}{|l|}{7} \\
\hline \multicolumn{7}{|l|}{$8 a$} \\
\hline \multicolumn{7}{|l|}{$8 b$} \\
\hline 9 & & $-1.14(-6.01)$ & & & & \\
\hline \multicolumn{7}{|l|}{10} \\
\hline \multicolumn{7}{|l|}{11} \\
\hline \multicolumn{7}{|l|}{12} \\
\hline \multicolumn{7}{|l|}{13} \\
\hline \multicolumn{7}{|l|}{14} \\
\hline \multicolumn{7}{|l|}{15} \\
\hline \multicolumn{7}{|l|}{$16 a$} \\
\hline \multicolumn{7}{|l|}{$16 \mathrm{~b}$} \\
\hline \multicolumn{7}{|l|}{$16 c$} \\
\hline \multicolumn{7}{|l|}{17} \\
\hline 18 & \begin{tabular}{|l|}
$-.779(-2.39)$ \\
\end{tabular} & $-.779(-2.39)$ & & & & \\
\hline \multicolumn{7}{|l|}{19} \\
\hline \multicolumn{7}{|l|}{20} \\
\hline \multicolumn{7}{|l|}{21} \\
\hline 22 & & & & & & \\
\hline $23 a$ & & & & & & \\
\hline $23 b$ & & & & & & \\
\hline 24 & & & & & & \\
\hline 25 & & & & & & \\
\hline 26 & & & & & $1.64(2.99)$ & \\
\hline 27 & & & & & & \\
\hline 28 & & $-1.01(-2.82)$ & & & & \\
\hline 29 & & & $615(2.88)$ & $.615(2.88)$ & & \\
\hline 30 & & & & & & \\
\hline
\end{tabular}

- positive coefficient

- negative coefficient

"snow" - "snowing weather" indicator variable

"clear" - "clear weather" indicator variable

"clo" _ - "cloudy weather" indicator variable 
Table C.5 (Continued)

\begin{tabular}{|c|c|c|c|c|c|c|}
\hline \multirow{3}{*}{ \# } & \multicolumn{6}{|c|}{ Coefficient (t-ratio) } \\
\hline & \multicolumn{2}{|c|}{ rain $\left[\mathrm{X}_{15}\right]$} & \multicolumn{2}{|c|}{ soil $\left[X_{15}\right]$} & \multicolumn{2}{|c|}{ dry $\left[\mathrm{X}_{16}\right]$} \\
\hline & fatality & injury & fatality & injury & fatality & injury \\
\hline 1 & & & & & & \\
\hline 2 & $3.23(2.57)$ & & & & & \\
\hline 3 & & & & & & \\
\hline 4 & & & & & & \\
\hline 5 & & & & & & \\
\hline 6 & & & & & & \\
\hline 7 & & $.644(2.58)$ & & & & \\
\hline $8 a$ & & & $1.89(1.99)$ & $1.89(1.99)$ & & \\
\hline $8 b$ & & & & & & \\
\hline 9 & & & & & & \\
\hline 10 & & & & & & \\
\hline 11 & & & & & & \\
\hline 12 & & & & & & \\
\hline 13 & & & & & & \\
\hline 14 & & & & & & \\
\hline 15 & & & & & & \\
\hline $16 a$ & & & & & & \\
\hline $16 \mathrm{~b}$ & & & & & & \\
\hline $16 c$ & & & & & & \\
\hline 17 & & & & & & \\
\hline 18 & & & & & & \\
\hline 19 & & & & & & \\
\hline 20 & & & & & & \\
\hline 21 & & & & & & \\
\hline 22 & & & & & & \\
\hline $23 a$ & & & & & & \\
\hline $23 b$ & & & & & & \\
\hline 24 & & & & & $.861(2.52)$ & $.324(5.05)$ \\
\hline 25 & & & & & & \\
\hline 26 & & & & & & \\
\hline 27 & & & & & & \\
\hline 28 & & & & & & \\
\hline 29 & & & & & & \\
\hline 30 & & & & & & \\
\hline
\end{tabular}

- positive coefficient

- negative coefficient

"rain" - "rainy weather" indicator variable

"soil" _ _ "blowing sand OR soil OR snow" indicator variable

"dry" - - "roadway surface is dry" indicator variable 
Table C.5 (Continued)

\begin{tabular}{|c|c|c|c|c|c|c|}
\hline \multirow{3}{*}{ \# } & \multicolumn{6}{|c|}{ Coefficient (t-ratio) } \\
\hline & \multicolumn{2}{|c|}{ slush $\left[\mathrm{X}_{16}\right]$} & \multicolumn{2}{|c|}{ driv $\left[\mathrm{X}_{17}\right]$} & \multicolumn{2}{|c|}{ nomed $\left[X_{17}\right]$} \\
\hline & fatality & injury & fatality & injury & fatality & injury \\
\hline 1 & & & & & & $.304(2.60)$ \\
\hline 2 & & & & & & \\
\hline 3 & $-1.46(-2.48)$ & $-.430(-4.63)$ & & & & \\
\hline 4 & & & & & & \\
\hline 5 & & & & & & \\
\hline 6 & & & & & & \\
\hline 7 & & & & & & \\
\hline $8 a$ & $2.70(2.18)$ & & & & & \\
\hline $8 b$ & & & & & & \\
\hline 9 & & & & & & \\
\hline 10 & & & & & & \\
\hline 11 & & & & & & \\
\hline 12 & & & & & & \\
\hline 13 & & & & & & \\
\hline 14 & & & & & & \\
\hline 15 & & & & & & \\
\hline $16 a$ & & & & $-.387(-3.09)$ & & \\
\hline $16 \mathrm{~b}$ & & & & & & \\
\hline $16 c$ & & & & & & \\
\hline 17 & & & & & & \\
\hline 18 & & & & & & \\
\hline 19 & & & & & & \\
\hline 20 & & & $-1.14(-2.38)$ & $-1.14(-2.38)$ & & \\
\hline 21 & $-.713(-2.67)$ & $-.713(-2.67)$ & & & & \\
\hline 22 & $-.236(-2.84)$ & $-.236(-2.84)$ & & & & $-.112(-3.54)$ \\
\hline $23 a$ & & & & & & \\
\hline $23 b$ & & & & & & \\
\hline 24 & & & $.361(6.27)$ & $.361(6.27)$ & & \\
\hline 25 & & & $-1.66(-2.19)$ & & & \\
\hline 26 & & & & & & \\
\hline 27 & & & & & & \\
\hline 28 & & $-.870(-2.97)$ & & & & \\
\hline 29 & & & & & & \\
\hline 30 & $-.779(-2.16)$ & $-.779(-2.16)$ & & & $-1.62(-2.03)$ & \\
\hline
\end{tabular}

- positive coefficient

- negative coefficient

"slush" - "roadway surface is covered by snow/slush" indicator variable

"driv" _ - "road median is a drivable" indicator variable

"nomed" - "no median" indicator variable 
Table C.5 (Continued)

\begin{tabular}{|c|c|c|c|c|c|c|}
\hline \multirow{3}{*}{ \# } & \multicolumn{6}{|c|}{ Coefficient (t-ratio) } \\
\hline & \multicolumn{2}{|c|}{ curb $\left[\mathrm{X}_{17}\right]$} & \multicolumn{2}{|c|}{ nojun $\left[\mathrm{X}_{18}\right]$} & \multicolumn{2}{|c|}{ way4 $\left[\mathrm{X}_{18}\right]$} \\
\hline & fatality & injury & fatality & injury & fatality & injury \\
\hline 1 & & & & & & $.240(2.29)$ \\
\hline 2 & & & & & & $699(2.81)$ \\
\hline 3 & & & & & & \\
\hline 4 & & & & & $.542(3.93)$ & $.542(3.93)$ \\
\hline 5 & & & & & & \\
\hline 6 & & & & & & \\
\hline 7 & & & $.498(2.11)$ & $.498(2.11)$ & & \\
\hline $8 a$ & & & & & & \\
\hline $8 b$ & & & & & & \\
\hline 9 & & & & $-.367(-2.09)$ & & \\
\hline 10 & & & & & & \\
\hline 11 & & & & & & \\
\hline 12 & & & & & & \\
\hline 13 & & & & & & \\
\hline 14 & & & & & & \\
\hline 15 & & & & & & \\
\hline 16a & & & & & & \\
\hline $16 \mathrm{~b}$ & $.407(2.67)$ & $.407(2.67)$ & & & & \\
\hline $16 c$ & & & & & & \\
\hline 17 & & & & & & \\
\hline 18 & & & & & & \\
\hline 19 & & & $-.278(-2.82)$ & & & \\
\hline 20 & & & & & & \\
\hline 21 & $-1.21(-3.07)$ & $-1.21(-3.07)$ & & & & \\
\hline 22 & & & $-.371(-11.3)$ & $-.371(-11.3)$ & & \\
\hline 23a & & & $-.472(-2.75)$ & & & \\
\hline $23 b$ & & & & & & \\
\hline 24 & & & & & $.298(3.41)$ & $.298(3.41)$ \\
\hline 25 & & & & & & \\
\hline 26 & & & & & $.517(2.16)$ & $517(2.16)$ \\
\hline 27 & & & & & & \\
\hline 28 & & & $-.182(-2.44)$ & $-.182(-2.44)$ & & \\
\hline 29 & & & & & $.441(2.17)$ & $441(2.17)$ \\
\hline 30 & & & & & & \\
\hline
\end{tabular}

- positive coefficient

- negative coefficient

"curb" - "road median is curbed" indicator variable

"nojun" - "no road junction at the accident location" indicator variable

"way4" - "accident location is at a 4-way intersection" indicator variable 
Table C.5 (Continued)

\begin{tabular}{|c|c|c|c|c|c|c|}
\hline \multirow{3}{*}{ \# } & \multicolumn{6}{|c|}{ Coefficient (t-ratio) } \\
\hline & \multicolumn{2}{|c|}{$\mathrm{T}\left[\mathrm{X}_{18}\right]$} & \multicolumn{2}{|c|}{ ramp $\left[\mathrm{X}_{18}\right]$} & \multicolumn{2}{|c|}{ curve $\left[\mathrm{X}_{19}\right]$} \\
\hline & fatality & injury & fatality & injury & fatality & injury \\
\hline \multicolumn{7}{|l|}{1} \\
\hline \multirow{2}{*}{\multicolumn{7}{|c|}{$\frac{2}{3}$}} \\
\hline & & & & & & \\
\hline \multicolumn{7}{|l|}{4} \\
\hline \multirow{2}{*}{\multicolumn{7}{|c|}{$\begin{array}{l}5 \\
6\end{array}$}} \\
\hline & & & & & & \\
\hline \multicolumn{7}{|l|}{7} \\
\hline \multicolumn{7}{|l|}{$8 a$} \\
\hline \multirow{2}{*}{\multicolumn{7}{|c|}{8}} \\
\hline & & & & & & \\
\hline 10 & & & $-.394(-2.28)$ & $-.394(-2.28)$ & & \\
\hline \multicolumn{7}{|l|}{11} \\
\hline \multicolumn{7}{|c|}{12} \\
\hline \multirow{2}{*}{\multicolumn{7}{|c|}{$\frac{13}{14}$}} \\
\hline 14 & & & & & & \\
\hline \multicolumn{7}{|c|}{15} \\
\hline \multicolumn{7}{|c|}{$16 a$} \\
\hline \multirow{2}{*}{\multicolumn{7}{|c|}{$\frac{16 \mathrm{~b}}{16 c}$}} \\
\hline & & & & & & \\
\hline \multicolumn{7}{|c|}{17} \\
\hline \multicolumn{7}{|l|}{18} \\
\hline \multicolumn{7}{|c|}{19} \\
\hline 20 & & $1.82(2.83)$ & & & & \\
\hline 21 & & & & & $.311(2.30)$ & $311(2.30)$ \\
\hline \multicolumn{7}{|c|}{22} \\
\hline \multicolumn{7}{|c|}{ 23a } \\
\hline \multirow{2}{*}{\multicolumn{7}{|c|}{$23 b$}} \\
\hline & & & & & & \\
\hline \multicolumn{7}{|c|}{25} \\
\hline \multicolumn{7}{|l|}{26} \\
\hline 27 & & & & & & $.278(2.01)$ \\
\hline \multirow{2}{*}{\multicolumn{7}{|c|}{28}} \\
\hline & & & & & & \\
\hline 30 & & & & & & \\
\hline
\end{tabular}

- positive coefficient

- negative coefficient

"T" - "accident location is at a T-intersection" indicator variable

"ramp" - "accident location is near or on a ramp" indicator variable

"curve" - "road is at curve" indicator variable 
Table C.5 (Continued)

\begin{tabular}{|c|c|c|c|c|c|c|}
\hline \multirow{3}{*}{ \# } & \multicolumn{6}{|c|}{ Coefficient (t-ratio) } \\
\hline & \multicolumn{2}{|c|}{$\operatorname{sg}\left[X_{19}\right]$} & \multicolumn{2}{|c|}{$\mathrm{sl}\left[\mathrm{X}_{19}\right]$} & \multicolumn{2}{|c|}{$\operatorname{str}\left[\mathrm{X}_{19}\right]$} \\
\hline & fatality & injury & fatality & injury & fatality & injury \\
\hline \multirow{2}{*}{\multicolumn{7}{|c|}{1}} \\
\hline & \multicolumn{6}{|c|}{2} \\
\hline 3 & & & & & $-.176(-2.93)$ & $-.176(-2.93)$ \\
\hline \multicolumn{7}{|l|}{4} \\
\hline \multicolumn{7}{|l|}{5} \\
\hline \multicolumn{7}{|l|}{6} \\
\hline \multirow{2}{*}{\multicolumn{7}{|c|}{$\frac{7}{8 a}$}} \\
\hline & & & & & & \\
\hline \multicolumn{7}{|c|}{$-1.39(-2.36)-1.39(-2.36)$} \\
\hline 9 & & & $-.256(-2.33)$ & $-.256(-2.33)$ & & \\
\hline \multicolumn{7}{|l|}{10} \\
\hline \multirow{2}{*}{\multicolumn{7}{|c|}{$\frac{11}{12}$}} \\
\hline & & & & & & \\
\hline \multicolumn{7}{|l|}{13} \\
\hline \multicolumn{7}{|c|}{14} \\
\hline 15 & & & & & $-1.20(-4.13)$ & $-.291(-3.12)$ \\
\hline \multicolumn{7}{|c|}{$16 \mathrm{a}$} \\
\hline \multirow{2}{*}{\multicolumn{7}{|c|}{$\frac{16 b}{16 c}$}} \\
\hline \multirow{2}{*}{\multicolumn{7}{|c|}{17}} \\
\hline & & & & & & \\
\hline \multicolumn{7}{|l|}{18} \\
\hline \multicolumn{7}{|c|}{19} \\
\hline \multirow{2}{*}{\multicolumn{7}{|c|}{$\frac{20}{21}$}} \\
\hline & & & & & & \\
\hline \multicolumn{7}{|c|}{22} \\
\hline \multirow{2}{*}{\multicolumn{7}{|c|}{$\frac{23 a}{23 h}$}} \\
\hline & \multicolumn{6}{|c|}{$23 b$} \\
\hline 24 & & & - $173(2.68)$ & . $173(2.68)$ & & \\
\hline \multicolumn{7}{|c|}{25} \\
\hline \multirow{2}{*}{\multicolumn{7}{|c|}{$\begin{array}{l}26 \\
27\end{array}$}} \\
\hline & & & & & & \\
\hline 28 & & & & & & \\
\hline 29 & & & & & & \\
\hline 30 & & & & & & \\
\hline
\end{tabular}

- positive coefficient

- negative coefficient

"sg" - - "road is straight AND at grade" indicator variable

"sl" _ - "road is straight AND level" indicator variable

"str" _ - "road is straight" indicator variable 
Table C.5 (Continued)

\begin{tabular}{|c|c|c|c|c|c|c|}
\hline \multirow{3}{*}{ \# } & \multicolumn{6}{|c|}{ Coefficient (t-ratio) } \\
\hline & \multicolumn{2}{|c|}{$\mathrm{cl}\left[\mathrm{X}_{19}\right]$} & \multicolumn{2}{|c|}{$\operatorname{sh}\left[\mathrm{X}_{19}\right]$} & \multicolumn{2}{|c|}{$\mathrm{cq}\left[\mathrm{X}_{19}\right]$} \\
\hline & fatality & injury & fatality & injury & fatality & injury \\
\hline 1 & & & $.329(2.05)$ & $.329(2.05)$ & & \\
\hline \multicolumn{7}{|l|}{2} \\
\hline 3 & & & & & & \\
\hline \multicolumn{7}{|l|}{4} \\
\hline \multirow{2}{*}{\multicolumn{7}{|c|}{$\frac{5}{6}$}} \\
\hline & & & & & & \\
\hline \multicolumn{7}{|l|}{7} \\
\hline \multicolumn{7}{|c|}{$8 a$} \\
\hline \multicolumn{4}{|c|}{9} & & & \\
\hline \multicolumn{7}{|c|}{10} \\
\hline 11 & & & & & $.999(2.40)$ & $.999(2.40)$ \\
\hline \multicolumn{7}{|c|}{12} \\
\hline \multicolumn{7}{|c|}{13} \\
\hline \multirow{2}{*}{\multicolumn{7}{|c|}{$\begin{array}{l}\mathbf{1 4} \\
15\end{array}$}} \\
\hline & & & & & & \\
\hline \multicolumn{7}{|c|}{$16 a$} \\
\hline \multirow{2}{*}{\multicolumn{7}{|c|}{$\frac{16 b}{16 c}$}} \\
\hline & & & & & & \\
\hline \multicolumn{7}{|c|}{17} \\
\hline \multicolumn{7}{|c|}{18} \\
\hline \multicolumn{7}{|c|}{$\frac{19}{20}$} \\
\hline \multirow{2}{*}{\multicolumn{7}{|c|}{$\frac{20}{21}$}} \\
\hline & & & & & & \\
\hline \multicolumn{7}{|c|}{22} \\
\hline \multicolumn{7}{|c|}{$23 a$} \\
\hline \multirow{2}{*}{\multicolumn{7}{|c|}{$\frac{23 b}{24}$}} \\
\hline & & & & & & \\
\hline \multicolumn{7}{|c|}{25} \\
\hline \multicolumn{7}{|c|}{$1.62(2.39)$} \\
\hline \multirow{2}{*}{\multicolumn{7}{|c|}{$\frac{27}{28}$}} \\
\hline & & & & & & \\
\hline \multicolumn{7}{|c|}{29} \\
\hline 30 & $2.73(4.20)$ & & & & & \\
\hline
\end{tabular}

- positive coefficient

- negative coefficient

"cl" - - "road is at-curve AND level" indicator variable

"sh" _ - "road is straight AND hillcrest" indicator variable

"cg" _ - "road is at-curve AND at grade" indicator variable 
Table C.5 (Continued)

\begin{tabular}{|c|c|c|c|c|c|c|}
\hline \multirow{3}{*}{ \# } & \multicolumn{6}{|c|}{ Coefficient (t-ratio) } \\
\hline & \multicolumn{2}{|c|}{$\operatorname{lev}\left[\mathrm{X}_{19}\right]$} & \multicolumn{2}{|c|}{ driver $\left[\mathrm{X}_{20}\right]$} & \multicolumn{2}{|c|}{$\operatorname{veh}\left[X_{20}\right]$} \\
\hline & fatality & injury & fatality & injury & fatality & injury \\
\hline \multirow{2}{*}{\multicolumn{7}{|c|}{$\begin{array}{l}1 \\
2\end{array}$}} \\
\hline & & & & & & \\
\hline \multicolumn{7}{|l|}{3} \\
\hline \multicolumn{7}{|l|}{4} \\
\hline \multicolumn{7}{|l|}{5} \\
\hline 6 & & & $1.27(4.94)$ & $1.27(4.94)$ & & \\
\hline \multicolumn{7}{|c|}{ 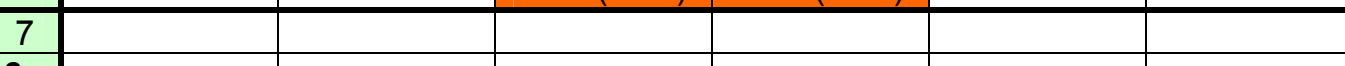 } \\
\hline \multicolumn{7}{|l|}{$8 a$} \\
\hline $8 b$ & & & $1.96(2.89)$ & $1.96(2.89)$ & & \\
\hline 9 & & & & $1.46(11.6)$ & & \\
\hline \multicolumn{7}{|l|}{10} \\
\hline \multicolumn{7}{|l|}{11} \\
\hline 12 & & $-.397(-2.76)$ & & $1.44(6.83)$ & & \\
\hline 13 & & & $.573(2.66)$ & $.573(2.66)$ & & \\
\hline \multirow{2}{*}{\multicolumn{7}{|c|}{$-1.58(-2.32)$}} \\
\hline & & & & & & \\
\hline \multicolumn{7}{|l|}{ 16a } \\
\hline \multirow{2}{*}{\multicolumn{7}{|c|}{$\frac{16 \mathrm{~b}}{1 \mathrm{c}}$}} \\
\hline & & & & & & \\
\hline \multicolumn{7}{|l|}{17} \\
\hline \multicolumn{7}{|l|}{18} \\
\hline \multicolumn{7}{|l|}{19} \\
\hline \multirow{2}{*}{\multicolumn{7}{|c|}{$145(971)$}} \\
\hline & & & & $1.45(9.71)$ & & \\
\hline \multicolumn{7}{|c|}{22} \\
\hline \multirow{2}{*}{\multicolumn{7}{|c|}{$23 a \mid$}} \\
\hline & & & & & & \\
\hline \multicolumn{7}{|c|}{$1.04(10.5)$} \\
\hline \multicolumn{7}{|l|}{25} \\
\hline \multirow{2}{*}{\multicolumn{7}{|c|}{$1.01(2.30)$}} \\
\hline & & & & & & \\
\hline 28 & & & $.569(2.37)$ & $.569(2.37)$ & & \\
\hline 29 & & & & & & \\
\hline 30 & & & & & & \\
\hline
\end{tabular}

- positive coefficient

- negative coefficient

"lev" _ - "road is at level" indicator variable

"driver" - "primary cause of accident is driver-related" indicator variable

"veh" - "primary cause of accident is vehicle-related" indicator variable 
Table C.5 (Continued)

\begin{tabular}{|c|c|c|c|c|c|c|}
\hline \multirow{3}{*}{ \# } & \multicolumn{6}{|c|}{ Coefficient (t-ratio) } \\
\hline & \multicolumn{2}{|c|}{ env $\left[X_{20}\right]$} & \multicolumn{2}{|c|}{$\mathrm{hl} 5\left[\mathrm{X}_{22}\right]$} & \multicolumn{2}{|c|}{$\mathrm{hl} 10\left[\mathrm{X}_{22}\right]$} \\
\hline & fatality & injury & fatality & injury & fatality & injury \\
\hline \multicolumn{7}{|l|}{1} \\
\hline 2 & & & & & $1.16(5.04)$ & $1.16(5.04)$ \\
\hline 3 & $-2.90(-6.30)$ & $-1.27(-19.7)$ & & & & \\
\hline \multicolumn{7}{|l|}{4} \\
\hline 5 & & & & & & \\
\hline 6 & & & & & $.804(4.26)$ & $.804(4.26)$ \\
\hline \multicolumn{7}{|l|}{7} \\
\hline \multicolumn{7}{|l|}{$8 a$} \\
\hline $8 b$ & & & & & & $1.10(3.20)$ \\
\hline 9 & & & & & & \\
\hline \multicolumn{7}{|l|}{10} \\
\hline \multicolumn{7}{|l|}{11} \\
\hline \multicolumn{7}{|l|}{12} \\
\hline 13 & & & & & $.631(6.37)$ & $631(6.37)$ \\
\hline \multicolumn{7}{|l|}{14} \\
\hline 15 & & $-1.78(-18.5)$ & & & & \\
\hline $16 \mathrm{a}$ & & & $.700(6.33)$ & $.700(6.33)$ & & \\
\hline $16 \mathrm{~b}$ & & & $.662(7.07)$ & $.662(7.07)$ & & \\
\hline $16 c$ & & & & $.442(2.64)$ & & \\
\hline \multicolumn{7}{|l|}{17} \\
\hline 18 & & $-1.62(-7.63)$ & $.887(6.27)$ & $.887(6.27)$ & & \\
\hline 19 & & & & & $.742(7.20)$ & . $742(7.20)$ \\
\hline \multicolumn{7}{|l|}{20} \\
\hline \multicolumn{7}{|l|}{21} \\
\hline 22 & & & & & $1.47(2.35)$ & $.777(22.8)$ \\
\hline $23 a$ & & & $.969(6.00)$ & $.969(6.00)$ & & \\
\hline $23 b$ & & & & $.629(3.08)$ & & \\
\hline 24 & & & & & $.872(13.6)$ & $.872(13.6)$ \\
\hline 25 & & & & & $.647(5.65)$ & \\
\hline \multicolumn{7}{|l|}{26} \\
\hline 27 & & $-1.59(-12.7)$ & & & & \\
\hline 28 & & & $.690(9.24)$ & $.690(9.24)$ & & \\
\hline 29 & & & $.974(4.62)$ & $.974(4.62)$ & & \\
\hline 30 & & $-1.34(-6.07)$ & $.965(6.09)$ & & & \\
\hline
\end{tabular}

- positive coefficient

- negative coefficient

"env" - "primary cause of accident is environment-related" indicator variable

"hl5" - "help arrived in 5 minutes or less after the crash" indicator variable

"hl10" - "help arrived in 10 minutes or less after the crash" indicator variable 
Table C.5 (Continued)

\begin{tabular}{|c|c|c|c|c|c|c|}
\hline \multirow{3}{*}{ \# } & \multicolumn{6}{|c|}{ Coefficient (t-ratio) } \\
\hline & \multicolumn{2}{|c|}{$\mathrm{hl} 20\left[\mathrm{X}_{22}\right]$} & \multicolumn{2}{|c|}{$\mathrm{hg} 30\left[\mathrm{X}_{22}\right]$} & \multicolumn{2}{|c|}{$\operatorname{car}\left[\mathrm{X}_{25}\right]$} \\
\hline & fatality & injury & fatality & injury & fatality & injury \\
\hline 1 & $2.27(2.21)$ & $.740(6.37)$ & & & & \\
\hline \multicolumn{7}{|l|}{2} \\
\hline 3 & $1.38(5.19)$ & .705 (12.3) & & & & \\
\hline 4 & $.758(3.78)$ & $.758(3.78)$ & & & & \\
\hline \multirow{2}{*}{\multicolumn{7}{|c|}{$\begin{array}{l}5 \\
6\end{array}$}} \\
\hline & & & & & & \\
\hline \multicolumn{7}{|l|}{7} \\
\hline \multicolumn{7}{|l|}{$8 a$} \\
\hline \multicolumn{7}{|c|}{$106(799)$} \\
\hline$\frac{9}{10}$ & $886(4.97)$ & $\frac{1.06(7.99)}{886(4.97)}$ & & & & \\
\hline 11 & $1.27(4.82)$ & $1.27(4.82)$ & & & & \\
\hline 12 & $.938(5.52)$ & $.938(5.52)$ & & & & \\
\hline \multicolumn{7}{|l|}{13} \\
\hline 14 & $1.55(5.23)$ & $1.55(5.23)$ & & & & \\
\hline 15 & $.872(9.39)$ & $.872(9.39)$ & & & & \\
\hline \multicolumn{7}{|c|}{$16 a$} \\
\hline \multirow{2}{*}{\multicolumn{7}{|c|}{$\frac{16 b}{16 c}$}} \\
\hline & & & & & & \\
\hline \multicolumn{7}{|l|}{17} \\
\hline \multicolumn{7}{|l|}{18} \\
\hline \multicolumn{7}{|l|}{19} \\
\hline 20 & $1.88(2.80)$ & $1.88(2.80)$ & & & & \\
\hline 21 & $.881(6.04)$ & $.881(6.04)$ & & & & \\
\hline \multicolumn{7}{|l|}{22} \\
\hline $23 a$ & & & & & $.575(2.87)$ & $.575(2.87)$ \\
\hline \multirow{2}{*}{\multicolumn{7}{|c|}{$\frac{23 b}{24}$}} \\
\hline & & & & & & \\
\hline \multicolumn{7}{|c|}{25} \\
\hline \multirow{2}{*}{$\begin{array}{l}26 \\
27 \\
\end{array}$} & & & $-2.40(-3.22)$ & $-2.40(-3.22)$ & & \\
\hline & $.851(6.24)$ & $.851(6.24)$ & & & & \\
\hline \multicolumn{7}{|l|}{28} \\
\hline 29 & & & & & & \\
\hline 30 & & & & & & \\
\hline
\end{tabular}

- positive coefficient

- negative coefficient

"hl20" - "help arrived in 20 minutes or less after the crash" indicator variable

"hg30" - "help arrived in more than 30 minutes after the crash" indicator variable

"car" - "the vehicle at fault is a car" indicator variable 
Table C.5 (Continued)

\begin{tabular}{|c|c|c|c|c|c|c|}
\hline \multirow{3}{*}{ \# } & \multicolumn{6}{|c|}{ Coefficient (t-ratio) } \\
\hline & & & \multicolumn{2}{|c|}{ heavy $\left[\mathrm{X}_{25}\right]$} & \multicolumn{2}{|c|}{ moto $\left[X_{25}\right]$} \\
\hline & fatality & injury & fatality & injury & fatality & injury \\
\hline \multirow{2}{*}{\multicolumn{7}{|c|}{1}} \\
\hline & & & & & & \\
\hline 3 & & & & & $2.93(12.2)$ & $2.93(12.2)$ \\
\hline \multicolumn{7}{|l|}{4} \\
\hline \multicolumn{7}{|l|}{5} \\
\hline 6 & & & & & $2.32(3.69)$ & $2.32(3.69)$ \\
\hline \multicolumn{7}{|c|}{$2.0<-10.04$} \\
\hline \multicolumn{7}{|l|}{$8 a$} \\
\hline \multirow{2}{*}{\multicolumn{7}{|c|}{$\begin{array}{c}80 \\
9\end{array}$}} \\
\hline & & & & & & \\
\hline \multicolumn{7}{|l|}{10} \\
\hline \multicolumn{7}{|l|}{11} \\
\hline 12 & & & & & $2.86(4.51)$ & $2.86(4.51)$ \\
\hline \multicolumn{7}{|l|}{13} \\
\hline 14 & & & $-1.19(-2.07)$ & $-1.19(-2.07)$ & & \\
\hline 15 & & & & & $3.09(9.82)$ & $3.09(9.82)$ \\
\hline \multicolumn{7}{|l|}{$16 a$} \\
\hline \multicolumn{7}{|l|}{$16 \mathrm{~b}$} \\
\hline \multirow{2}{*}{\multicolumn{7}{|c|}{\begin{tabular}{|c|}
$16 c$ \\
17
\end{tabular}}} \\
\hline & & & & & & \\
\hline 18 & & & & & $1.94(4.97)$ & $1.94(4.97)$ \\
\hline \multicolumn{7}{|l|}{19} \\
\hline \multicolumn{7}{|l|}{20} \\
\hline 21 & & & & & & $3.46(8.00)$ \\
\hline \multicolumn{7}{|l|}{22} \\
\hline \multirow{2}{*}{\multicolumn{7}{|c|}{$\frac{23 a}{23 b}$}} \\
\hline & & & & & & \\
\hline \multicolumn{7}{|c|}{$2.31(11.5)$} \\
\hline 25 & & $.550(-2.78)$ & & & & \\
\hline \multicolumn{7}{|l|}{26} \\
\hline 27 & & & & & $4.19(5.67)$ & $4.19(5.67)$ \\
\hline \multicolumn{7}{|l|}{28} \\
\hline 29 & & & & & & \\
\hline 30 & & & & & & \\
\hline
\end{tabular}

- positive coefficient

- negative coefficient

"SUV" - "the vehicle at fault is a SUV" indicator variable

"heavy" - "the vehicle at fault is a truck or a tractor" indicator variable

"moto" - "the vehicle at fault is a motorcycle" indicator variable 
Table C.5 (Continued)

\begin{tabular}{|c|c|c|c|c|c|c|}
\hline \multirow{3}{*}{ \# } & \multicolumn{6}{|c|}{ Coefficient (t-ratio) } \\
\hline & \multicolumn{2}{|c|}{ pickup $\left[\mathrm{X}_{25}\right]$} & \multicolumn{2}{|c|}{$\operatorname{van}\left[\mathrm{X}_{25}\right]$} & \multicolumn{2}{|c|}{ trac1 $\left[\mathrm{X}_{25}\right]$} \\
\hline & fatality & injury & fatality & injury & fatality & injury \\
\hline \multicolumn{7}{|l|}{1} \\
\hline \multicolumn{7}{|l|}{2} \\
\hline \multicolumn{7}{|l|}{3} \\
\hline \multicolumn{7}{|l|}{4} \\
\hline \multicolumn{7}{|l|}{5} \\
\hline \multicolumn{7}{|l|}{6} \\
\hline \multicolumn{7}{|l|}{7} \\
\hline \multicolumn{7}{|l|}{$8 a$} \\
\hline \multicolumn{7}{|l|}{$8 b$} \\
\hline \multicolumn{7}{|l|}{9} \\
\hline \multicolumn{7}{|l|}{10} \\
\hline \multicolumn{7}{|l|}{11} \\
\hline \multicolumn{7}{|l|}{12} \\
\hline \multicolumn{7}{|l|}{13} \\
\hline \multicolumn{7}{|l|}{14} \\
\hline \multicolumn{7}{|l|}{15} \\
\hline \multicolumn{7}{|l|}{$16 a$} \\
\hline $16 \mathrm{~b}$ & & & $.300(2.08)$ & $300(2.08)$ & & \\
\hline $16 c$ & & $-.351(-2.11)$ & & & & \\
\hline \multicolumn{7}{|l|}{17} \\
\hline \multicolumn{7}{|l|}{18} \\
\hline 19 & & & $-.428(-2.20)$ & $-.428(-2.20)$ & & \\
\hline \multicolumn{7}{|l|}{20} \\
\hline \multicolumn{7}{|l|}{21} \\
\hline \multicolumn{7}{|l|}{22} \\
\hline $23 a$ & & & & & & \\
\hline $23 b$ & & & & & $-.905(-2.71)$ & $-.905(-2.71)$ \\
\hline 24 & & & & & & \\
\hline 25 & & & & & & \\
\hline 26 & & & & & & \\
\hline 27 & & & & & & \\
\hline 28 & & $-.291(-2.83)$ & & & & \\
\hline 29 & & & & & & \\
\hline 30 & & & & & & \\
\hline
\end{tabular}

- positive coefficient

- negative coefficient

"pickup" - "the vehicle at fault is a pickup" indicator variable

"van" - "the vehicle at fault is a van" indicator variable

"trac1" - "the vehicle at fault is a tractor OR one semi-trailer" indicator variable 
Table C.5 (Continued)

\begin{tabular}{|c|c|c|c|c|c|c|}
\hline \multirow{3}{*}{ \# } & \multicolumn{6}{|c|}{ Coefficient (t-ratio) } \\
\hline & \multicolumn{2}{|c|}{ vage $\left[X_{26}\right]$} & \multicolumn{2}{|c|}{ voldg $\left[\mathrm{X}_{26}\right]$} & \multicolumn{2}{|c|}{$v 7 g\left[X_{26}\right]$} \\
\hline & fatality & injury & fatality & injury & fatality & injury \\
\hline 1 & $128(3.91)$ & & & & & \\
\hline \multicolumn{7}{|l|}{2} \\
\hline 3 & $.0311(6.59)$ & $.0311(6.59)$ & & & & \\
\hline 4 & & $.0420(3.14)$ & & & & \\
\hline 5 & & & $-1.25(-3.74)$ & $-1.25(-3.74)$ & & \\
\hline 6 & & & & & $-.532(-2.47)$ & $-.532(-2.47)$ \\
\hline \multicolumn{7}{|l|}{7} \\
\hline $8 a$ & & & & & $-.797(-2.55)$ & \begin{tabular}{|l}
$-.797(-2.55)$ \\
\end{tabular} \\
\hline \multicolumn{7}{|l|}{$8 b$} \\
\hline 9 & & & & $.228(2.04)$ & & \\
\hline \multicolumn{7}{|l|}{10} \\
\hline \multicolumn{7}{|l|}{11} \\
\hline \multicolumn{7}{|l|}{12} \\
\hline \multicolumn{7}{|l|}{13} \\
\hline 14 & $.0601(3.35)$ & $.0601(3.35)$ & & & & \\
\hline 15 & $.0332(4.37)$ & $.0332(4.37)$ & & & & \\
\hline \multicolumn{7}{|c|}{$16 a$} \\
\hline \multicolumn{7}{|c|}{$16 \mathrm{~b}$} \\
\hline \multicolumn{7}{|c|}{$16 c$} \\
\hline \multicolumn{7}{|l|}{17} \\
\hline \multicolumn{7}{|l|}{18} \\
\hline \multicolumn{7}{|l|}{19} \\
\hline \multicolumn{7}{|l|}{20} \\
\hline 21 & $.0216(2.02)$ & $.0216(2.02)$ & & & & \\
\hline 22 & & & $1.40(2.74)$ & $.110(3.72)$ & & \\
\hline \multicolumn{7}{|c|}{$23 a$} \\
\hline \multicolumn{7}{|c|}{$23 b$} \\
\hline 24 & & & & $.315(5.53)$ & & \\
\hline \multicolumn{7}{|l|}{25} \\
\hline \multicolumn{7}{|l|}{26} \\
\hline 27 & $.0329(3.27)$ & $.0329(3.27)$ & & & & \\
\hline \multicolumn{7}{|l|}{28} \\
\hline \multicolumn{7}{|l|}{29} \\
\hline 30 & & & & & & \\
\hline
\end{tabular}

- positive coefficient

- negative coefficient

"vage" - "age (in years) of the vehicle at fault" quantitative variable

"voldg" - "the vehicle at fault is more than 7 years old" indicator variable

"v7g" - "age of the vehicle at fault is $\geq 3$ and $\leq 7$ years" indicator variable 
Table C.5 (Continued)

\begin{tabular}{|c|c|c|c|c|c|c|}
\hline \multirow{3}{*}{ \# } & \multicolumn{6}{|c|}{ Coefficient (t-ratio) } \\
\hline & \multicolumn{2}{|c|}{ v3g $\left[X_{26}\right]$} & \multicolumn{2}{|c|}{$\mathrm{X}_{27}$} & \multicolumn{2}{|c|}{ Ind $\left[\mathrm{X}_{28}\right]$} \\
\hline & fatality & injury & fatality & injury & fatality & injury \\
\hline \multirow{2}{*}{\multicolumn{7}{|c|}{$\frac{1}{2}$}} \\
\hline & 2 & & & & & \\
\hline 3 & & & $.0382(3.47)$ & $.0382(3.47)$ & & \\
\hline \multicolumn{7}{|l|}{4} \\
\hline \multirow{2}{*}{\multicolumn{7}{|c|}{6}} \\
\hline & & & & & & \\
\hline \multicolumn{7}{|l|}{7} \\
\hline \multirow{2}{*}{\multicolumn{7}{|c|}{$.303(2.37)$}} \\
\hline & & & & & & \\
\hline \multicolumn{7}{|l|}{10} \\
\hline \multicolumn{7}{|l|}{11} \\
\hline 12 & & & & & & \\
\hline \multicolumn{6}{|l|}{13} & \\
\hline \multicolumn{7}{|l|}{14} \\
\hline \multicolumn{7}{|l|}{15} \\
\hline \multicolumn{7}{|l|}{$16 a$} \\
\hline $16 \mathrm{~b}$ & $-.406(-2.71)$ & $-.406(-2.71)$ & $-.142(-2.15)$ & $-.142(-2.15)$ & $404(2.04)$ & $404(2.04)$ \\
\hline \multirow{2}{*}{\multicolumn{7}{|c|}{$\frac{16 c}{17}$}} \\
\hline & & & & & & \\
\hline \multicolumn{7}{|l|}{18} \\
\hline 19 & & & $-.238(-3.14)$ & $-.238(-3.14)$ & & \\
\hline \multicolumn{7}{|l|}{20} \\
\hline 21 & & & $.133(2.26)$ & $.133(2.26)$ & & \\
\hline 22 & & & $-.0628(-2.89)$ & $-.0628(-2.89)$ & & \\
\hline \multicolumn{7}{|l|}{ 23a } \\
\hline \multicolumn{7}{|l|}{$23 b$} \\
\hline 24 & & & $.0817(3.11)$ & $.0817(3.11)$ & $477(4.10)$ & $477(4.10)$ \\
\hline \multicolumn{7}{|l|}{25} \\
\hline \multirow{2}{*}{\multicolumn{7}{|c|}{$\frac{26}{27}$}} \\
\hline & & & & & & \\
\hline \multicolumn{7}{|l|}{\begin{tabular}{l|}
28 \\
0
\end{tabular}} \\
\hline 29 & & & & & & \\
\hline 30 & & & & & & \\
\hline
\end{tabular}

- positive coefficient

- negative coefficient

"v3g" - "age of the vehicle at fault is $>1$ and $\leq 3$ years" indicator variable

"X $\mathrm{X}_{27}$ " - "number of occupants in the vehicle at fault" quantitative variable

"Ind" _ - "license state of the vehicle at fault is Indiana" indicator variable 
Table C.5 (Continued)

\begin{tabular}{|c|c|c|c|c|c|c|}
\hline \multirow{3}{*}{ \# } & \multicolumn{6}{|c|}{ Coefficient (t-ratio) } \\
\hline & \multicolumn{2}{|c|}{ othUS $\left[\mathrm{X}_{28}\right]$} & \multicolumn{2}{|c|}{$\operatorname{lnm}\left[\mathrm{X}_{30}\right]$} & \multicolumn{2}{|c|}{$\mathrm{r} 22\left[\mathrm{X}_{30}\right]$} \\
\hline & fatality & injury & fatality & injury & fatality & injury \\
\hline \multicolumn{7}{|l|}{1} \\
\hline \multirow{2}{*}{\multicolumn{7}{|c|}{$\frac{2}{3}$}} \\
\hline & & & & & & \\
\hline \multicolumn{7}{|l|}{4} \\
\hline \multicolumn{7}{|l|}{5} \\
\hline 6 & $3.60(2.82)$ & $3.60(2.82)$ & & & & \\
\hline \multicolumn{7}{|l|}{7} \\
\hline \multicolumn{7}{|l|}{$\frac{8 a}{8 b}$} \\
\hline \multicolumn{7}{|l|}{$\begin{array}{r}8 b \\
9\end{array}$} \\
\hline 10 & $-.970(-2.20)$ & $-.970(-2.20)$ & & & & \\
\hline 11 & $-.695(-2.28)$ & $-.695(-2.28)$ & & & & \\
\hline \multicolumn{7}{|l|}{12} \\
\hline 13 & & & & & & $.369(3.18)$ \\
\hline \multirow{2}{*}{\multicolumn{7}{|c|}{$\begin{array}{l}14 \\
15\end{array}$}} \\
\hline & & & & & & \\
\hline \multicolumn{7}{|l|}{$16 \mathrm{a}$} \\
\hline \multicolumn{7}{|l|}{$\frac{16 \mathrm{~b}}{16 \mathrm{c}}$} \\
\hline \multirow{2}{*}{\multicolumn{7}{|c|}{17}} \\
\hline & & & & & & \\
\hline 18 & $-1.12(-2.26)$ & $-1.12(-2.26)$ & $.414(2.85)$ & $.414(2.85)$ & & \\
\hline \multicolumn{7}{|c|}{ 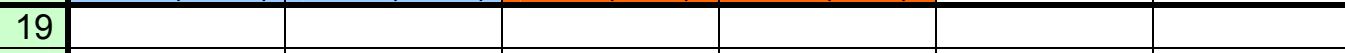 } \\
\hline \multirow{2}{*}{\multicolumn{7}{|c|}{$\frac{20}{21}$}} \\
\hline & & & & & & \\
\hline \multicolumn{7}{|l|}{22} \\
\hline \multicolumn{7}{|l|}{ 23a } \\
\hline \multirow{2}{*}{\multicolumn{7}{|c|}{24}} \\
\hline & & & & & & \\
\hline \multicolumn{7}{|l|}{25} \\
\hline \multicolumn{7}{|l|}{26} \\
\hline \multirow{2}{*}{\multicolumn{7}{|c|}{28}} \\
\hline & & & & & & \\
\hline 29 & & & & & & \\
\hline 30 & & & & & & \\
\hline
\end{tabular}

- positive coefficient

- negative coefficient

"othUS" - "license state of the vehicle at fault is a U.S. state except Indiana and its neighboring states (IL, $\mathrm{KY}, \mathrm{OH}, \mathrm{MI}$ )" indicator variable

"Inm" _ "road traveled by the vehicle at fault is multi-lane" indicator variable

"r22" - - "road traveled by the vehicle at fault is two-lane AND two-way" indicator variable 
Table C.5 (Continued)

\begin{tabular}{|c|c|c|c|c|c|c|}
\hline \multirow{3}{*}{ \# } & \multicolumn{6}{|c|}{ Coefficient (t-ratio) } \\
\hline & \multicolumn{2}{|c|}{ rmu22 $\left[X_{30}\right]$} & \multicolumn{2}{|c|}{ rmd2 $\left[\mathrm{X}_{30}\right]$} & \multicolumn{2}{|c|}{ priv $\left[\mathrm{X}_{30}\right]$} \\
\hline & fatality & injury & fatality & injury & fatality & injury \\
\hline \multirow{2}{*}{\multicolumn{7}{|c|}{1}} \\
\hline & & & & & & \\
\hline \multicolumn{7}{|l|}{3} \\
\hline \multicolumn{7}{|l|}{4} \\
\hline 5 & & & & $2.06(2.99)$ & & \\
\hline \multicolumn{7}{|l|}{6} \\
\hline \multicolumn{7}{|l|}{7} \\
\hline \multicolumn{7}{|l|}{$\frac{8 a}{8 b}$} \\
\hline \multicolumn{7}{|l|}{ 9 } \\
\hline 10 & $3.89(3.15)$ & & & & & \\
\hline \multicolumn{7}{|l|}{11} \\
\hline \multicolumn{7}{|l|}{12} \\
\hline \multicolumn{7}{|l|}{13} \\
\hline \multirow{2}{*}{\multicolumn{7}{|c|}{$\begin{array}{l}\mathbf{1 4} \\
15\end{array}$}} \\
\hline & & & & & & \\
\hline \multirow{2}{*}{\multicolumn{7}{|c|}{$\frac{16 \mathrm{a}}{16 \mathrm{~b}}$}} \\
\hline \multirow{2}{*}{\multicolumn{7}{|c|}{$\begin{array}{l}16 \mathrm{~b} \\
16 c\end{array}$}} \\
\hline & & & & & & \\
\hline \multicolumn{7}{|l|}{17} \\
\hline \multicolumn{7}{|l|}{18} \\
\hline \multirow{2}{*}{\multicolumn{7}{|c|}{$\frac{19}{20}$}} \\
\hline & & & & & & \\
\hline \multicolumn{7}{|r|}{$-1.14(-3.10)$} \\
\hline 22 & & & & & $-.489(-3.05)$ & $-.489(-3.05)$ \\
\hline $23 a$ & & & $374(1.98)$ & $.374(1.98)$ & & \\
\hline \multicolumn{7}{|l|}{$23 b$} \\
\hline 24 & & & & & & $-.490(-4.09)$ \\
\hline \multicolumn{7}{|l|}{25} \\
\hline \multirow{2}{*}{\multicolumn{7}{|c|}{$\begin{array}{l}26 \\
26\end{array}$}} \\
\hline & & & & & & \\
\hline \multicolumn{7}{|l|}{28} \\
\hline 29 & & & & & & \\
\hline 30 & & & & & & \\
\hline
\end{tabular}

- positive coefficient

- negative coefficient

\footnotetext{
"rmu22" - "road traveled by the vehicle at fault is multi-lane AND undivided two-way left" indicator variable

"rmd2" - "road traveled by the vehicle at fault is multi-lane AND divided three or more" indicator variable

"priv" _ - "road traveled by the vehicle at fault is a private drive" indicator variable
} 
Table C.5 (Continued)

\begin{tabular}{|c|c|c|c|c|c|c|}
\hline \multirow{3}{*}{ \# } & \multicolumn{6}{|c|}{ Coefficient (t-ratio) } \\
\hline & \multicolumn{2}{|c|}{ stopsig $\left[X_{31}\right]$} & \multicolumn{2}{|c|}{ nosig $\left[\mathrm{X}_{31}\right]$} & \multicolumn{2}{|c|}{ nopass $\left[X_{31}\right]$} \\
\hline & fatality & injury & fatality & injury & fatality & injury \\
\hline 1 & $.260(2.50)$ & $.260(2.50)$ & & & & \\
\hline \multicolumn{7}{|l|}{2} \\
\hline 3 & & & & & & \\
\hline \multicolumn{7}{|l|}{4} \\
\hline \multirow{2}{*}{\multicolumn{7}{|c|}{$\begin{array}{l}5 \\
6\end{array}$}} \\
\hline & & & & & & \\
\hline \multicolumn{7}{|l|}{7} \\
\hline \multicolumn{7}{|l|}{$8 a$} \\
\hline \multirow{2}{*}{\multicolumn{7}{|c|}{$\begin{array}{r}\boldsymbol{8 b} \\
9\end{array}$}} \\
\hline & & & & & & \\
\hline \multicolumn{7}{|l|}{10} \\
\hline \multicolumn{7}{|l|}{11} \\
\hline \multicolumn{7}{|l|}{12} \\
\hline 13 & $1.24(2.48)$ & $.444(3.02)$ & & & & \\
\hline \multicolumn{7}{|l|}{14} \\
\hline 15 & & & & & $.198(2.02)$ & $198(2.02)$ \\
\hline \multicolumn{7}{|l|}{$\frac{16 \mathrm{a}}{16 \mathrm{~b}}$} \\
\hline \multirow{2}{*}{\multicolumn{7}{|c|}{$\frac{16 \mathrm{~b}}{16 \mathrm{c}}$}} \\
\hline & & & & & & \\
\hline \multicolumn{7}{|c|}{$3.51(2.41)$} \\
\hline \multicolumn{7}{|l|}{18} \\
\hline \multicolumn{7}{|l|}{19} \\
\hline \multirow{2}{*}{\multicolumn{7}{|c|}{$\frac{20}{21}$}} \\
\hline & & & & & & \\
\hline \multirow{2}{*}{\multicolumn{6}{|c|}{$. .100(-2.11)--.100(-2.11)$}} & \\
\hline & & & & & & \\
\hline \multicolumn{7}{|l|}{$23 b$} \\
\hline \multicolumn{7}{|l|}{24} \\
\hline 25 & $.505(2.82)$ & $.505(2.82)$ & & & & \\
\hline \multicolumn{7}{|l|}{26} \\
\hline 27 & & & & $-.286(-2.45)$ & & \\
\hline \multicolumn{7}{|l|}{28} \\
\hline 29 & & & & & & \\
\hline 30 & & & & & & \\
\hline
\end{tabular}

- positive coefficient

- negative coefficient

"stopsig" - "traffic control device for the vehicle at fault is a «stop sign»" indicator variable

"nosig" - "no any traffic control device for the vehicle at fault" indicator variable

"nopass" - "traffic control device for the vehicle at fault is a «no passing zone»" indicator variable 
Table C.5 (Continued)

\begin{tabular}{|c|c|c|c|c|c|c|}
\hline \multirow{3}{*}{ \# } & \multicolumn{6}{|c|}{ Coefficient (t-ratio) } \\
\hline & \multicolumn{2}{|c|}{$\operatorname{sig}\left[\mathrm{X}_{31}\right]$} & \multicolumn{2}{|c|}{ other $\left[X_{31}\right]$} & \multicolumn{2}{|c|}{$X_{33}$} \\
\hline & fatality & injury & fatality & injury & fatality & injury \\
\hline 1 & & & & & $2.36(1.99)$ & $1.85(3.11)$ \\
\hline \multicolumn{7}{|l|}{2} \\
\hline 3 & & & $1.56(2.85)$ & $.631(2.52)$ & $2.24(5.03)$ & $1.00(3.88)$ \\
\hline \multirow{2}{*}{\multicolumn{7}{|c|}{4}} \\
\hline & & & & & & \\
\hline 6 & & & & & $2.81(2.36)$ & $2.81(2.36)$ \\
\hline \multicolumn{7}{|c|}{$-2=1$} \\
\hline $8 a$ & & & & & $6.11(4.48)$ & \\
\hline \multicolumn{7}{|l|}{$\begin{array}{r}8 \boldsymbol{b b} \\
9\end{array}$} \\
\hline 10 & & & & & $1.78(2.03)$ & $1.78(2.03)$ \\
\hline 11 & & & & & $4.51(4.33)$ & \\
\hline \multicolumn{7}{|l|}{12} \\
\hline 13 & & & & & $2.62(2.82)$ & \\
\hline 14 & & & & & $2.81(2.74)$ & \\
\hline 15 & & & & & $1.96(3.13)$ & \\
\hline \multirow{2}{*}{\multicolumn{7}{|c|}{$\frac{16 a}{16 b}$}} \\
\hline & & & & & & \\
\hline \multicolumn{7}{|c|}{$5.07(3.73)$} \\
\hline \multicolumn{7}{|l|}{$\frac{17}{18}$} \\
\hline 18 & & & & & & \\
\hline \multicolumn{7}{|l|}{19} \\
\hline \multicolumn{7}{|l|}{20} \\
\hline \multicolumn{7}{|l|}{21} \\
\hline 22 & & & & & $1.95(8.94)$ & $1.95(8.94)$ \\
\hline 23a & & & & & & $2.67(3.01)$ \\
\hline $23 b$ & $.268(2.44)$ & $.268(2.44)$ & & & $1.16(4.19)$ & $1.16(4.19)$ \\
\hline \multicolumn{7}{|l|}{25} \\
\hline \multirow{2}{*}{\multicolumn{7}{|c|}{$3.21(3.57)$}} \\
\hline & & & & & & \\
\hline 28 & & & & & $1.91(3.60)$ & $1.91(3.60)$ \\
\hline 29 & & & & & & \\
\hline 30 & & & & & & \\
\hline
\end{tabular}

- positive coefficient

- negative coefficient

"sig" _ - "traffic control device for the vehicle at fault is a signal" indicator variable

"other" - "traffic control device for the vehicle at fault is an «other regulatory sign or marking»" indicator variable

" $\mathrm{X}_{33}$ " $\quad$ - "at least one of the vehicles involved was on fire" indicator variable 
Table C.5 (Continued)

\begin{tabular}{|c|c|c|c|c|c|c|}
\hline \multirow{3}{*}{ \# } & \multicolumn{6}{|c|}{ Coefficient (t-ratio) } \\
\hline & \multicolumn{2}{|c|}{$X_{34}$} & \multicolumn{2}{|c|}{ age2 $\left[\mathrm{X}_{34}\right]$} & \multicolumn{2}{|c|}{ age3 $\left[X_{34}\right]$} \\
\hline & fatality & injury & fatality & injury & fatality & injury \\
\hline \multirow{2}{*}{\multicolumn{7}{|c|}{$\frac{1}{2}$}} \\
\hline & & & & & & \\
\hline 3 & $-.0071(3.93$ & & & & & \\
\hline \multicolumn{7}{|l|}{4} \\
\hline \multirow{2}{*}{\multicolumn{7}{|c|}{6}} \\
\hline & & & & & & \\
\hline \multicolumn{7}{|l|}{7} \\
\hline \multicolumn{7}{|l|}{$8 a$} \\
\hline \multirow{2}{*}{\multicolumn{7}{|c|}{$-.576(-3.67)$}} \\
\hline & & & & & & \\
\hline 10 & & & & & $-.377(-2.09)$ & $-.377(-2.09)$ \\
\hline \multicolumn{7}{|l|}{11} \\
\hline \multicolumn{7}{|l|}{12} \\
\hline \multirow{2}{*}{\multicolumn{7}{|c|}{$\frac{13}{14}$}} \\
\hline \multirow{2}{*}{\multicolumn{7}{|c|}{15}} \\
\hline & & & & & & \\
\hline \multicolumn{7}{|l|}{$\frac{16 a}{16 b}$} \\
\hline \multirow{2}{*}{\multicolumn{7}{|c|}{$\begin{array}{ll}16 b \\
16 c\end{array}$}} \\
\hline & & & & & & \\
\hline \multicolumn{7}{|l|}{17} \\
\hline \multicolumn{7}{|l|}{18} \\
\hline \multirow{2}{*}{\multicolumn{7}{|c|}{$\frac{19}{20}$}} \\
\hline \multirow{2}{*}{\multicolumn{7}{|c|}{$\begin{array}{l}20 \\
21\end{array}$}} \\
\hline & & & & & & \\
\hline \multirow{2}{*}{\multicolumn{7}{|c|}{$.0346(3.54) .00180(2.20)$}} \\
\hline & & & & & & \\
\hline \multicolumn{7}{|l|}{$23 b$} \\
\hline \multicolumn{7}{|c|}{$.381(2.20)$} \\
\hline \multirow{2}{*}{\multicolumn{7}{|c|}{$\frac{25}{26}$}} \\
\hline & & & & & & \\
\hline \multicolumn{7}{|l|}{27} \\
\hline \multicolumn{7}{|l|}{28} \\
\hline 29 & & & & & & \\
\hline 30 & & & & & & \\
\hline
\end{tabular}

- positive coefficient

- negative coefficient

" $\mathrm{X}_{34} " \quad-\quad$ - "age (in years) of the driver at fault" quantitative variable

"age2" - "age of the driver at fault is $\geq 24$ and $<30$ " indicator variable

"age3" - "age of the driver at fault is $\geq 30$ and $<40$ " indicator variable 
Table C.5 (Continued)

\begin{tabular}{|c|c|c|c|c|c|c|}
\hline \multirow{3}{*}{ \# } & \multicolumn{6}{|c|}{ Coefficient (t-ratio) } \\
\hline & \multicolumn{2}{|c|}{ age $5\left[X_{34}\right]$} & \multicolumn{2}{|c|}{$\mathrm{X}_{35}$} & \multicolumn{2}{|c|}{ oldvage $\left[\mathrm{X}_{26}\right]$} \\
\hline & fatality & injury & fatality & injury & fatality & injury \\
\hline 1 & $1.09(2.37)$ & & & & & \\
\hline 2 & & & & $.540(2.11)$ & & \\
\hline 3 & & & & $.290(5.39)$ & & \\
\hline 4 & & & $-.403(-2.51)$ & $-.403(-2.51)$ & & \\
\hline \multirow{2}{*}{\multicolumn{7}{|c|}{5}} \\
\hline & & & & & & \\
\hline \multicolumn{7}{|l|}{7} \\
\hline \multicolumn{7}{|l|}{$8 a$} \\
\hline \multirow{2}{*}{\multicolumn{7}{|c|}{$365(3.44)$}} \\
\hline & & & $.365(3.44)$ & $.365(3.44)$ & & \\
\hline \multirow{2}{*}{\multicolumn{7}{|c|}{$\begin{array}{l}10 \\
11\end{array}$}} \\
\hline \multicolumn{6}{|l|}{11} & \\
\hline 12 & & & & $.533(3.94)$ & & \\
\hline 13 & & & $-2.02(-2.69)$ & & & \\
\hline \multicolumn{7}{|l|}{14} \\
\hline 15 & & & $-.900(-2.42)$ & $.229(2.74)$ & & \\
\hline \multicolumn{7}{|l|}{$\frac{16 a}{16 a}$} \\
\hline \multicolumn{7}{|l|}{$16 \mathrm{~b}$} \\
\hline $16 c$ & & & & & & $.0428(2.73)$ \\
\hline \multicolumn{7}{|l|}{17} \\
\hline \multicolumn{7}{|l|}{18} \\
\hline \multicolumn{7}{|l|}{19} \\
\hline \multirow{2}{*}{\multicolumn{7}{|c|}{$-1.33(-2.11)$}} \\
\hline & & & & & & \\
\hline \multicolumn{7}{|l|}{22} \\
\hline \multicolumn{7}{|c|}{$-.556(-2.82)$} \\
\hline $23 b$ & & & & & & \\
\hline 24 & & & & .412(7.07) & & \\
\hline \multicolumn{7}{|l|}{25} \\
\hline \multirow{2}{*}{\multicolumn{7}{|c|}{$\begin{array}{l}26 \\
27\end{array}$}} \\
\hline & & & & & & \\
\hline \multicolumn{7}{|l|}{\begin{tabular}{l|}
28 \\
0
\end{tabular}} \\
\hline 29 & & & & & & \\
\hline 30 & & & & & & \\
\hline
\end{tabular}

- positive coefficient

- negative coefficient

\begin{tabular}{ll}
\hline "age5" & - "age of the driver at fault is $\geq 50$ years" indicator variable \\
"X & - "gender of the driver at fault: $1-$ female, $0-$ male" indicator variable \\
"oldvage" & - "age (in years) of the oldest vehicle involved" quantitative variable
\end{tabular} 
Table C.5 (Continued)

\begin{tabular}{|c|c|c|c|c|c|c|}
\hline \multirow{3}{*}{ \# } & \multicolumn{6}{|c|}{ Coefficient (t-ratio) } \\
\hline & \multicolumn{2}{|c|}{ voldo $\left[\mathrm{X}_{26}\right]$} & \multicolumn{2}{|c|}{$\operatorname{maxpass}\left[\mathrm{X}_{27}\right]$} & \multicolumn{2}{|c|}{ age5y $\left[X_{34}\right]$} \\
\hline & fatality & injury & fatality & injury & fatality & injury \\
\hline 1 & & & $-.691(-1.99)$ & $.133(3.60)$ & & \\
\hline \multicolumn{7}{|l|}{2} \\
\hline \multicolumn{7}{|l|}{3} \\
\hline 4 & & & $.186(2.71)$ & $.186(2.71)$ & & \\
\hline 5 & & & & $.863(2.60)$ & & \\
\hline \multicolumn{7}{|l|}{6} \\
\hline \multirow{2}{*}{\multicolumn{7}{|c|}{$\frac{7}{8 a}$}} \\
\hline & & & & & & \\
\hline \multicolumn{7}{|c|}{$-1.50(-3.39)$} \\
\hline 9 & & & & & & \\
\hline \multicolumn{7}{|c|}{$.141(2.42)$} \\
\hline \multicolumn{7}{|l|}{11} \\
\hline \multirow{2}{*}{\multicolumn{5}{|c|}{$\frac{12}{13}$}} & & \\
\hline & & & & & & \\
\hline & \multicolumn{2}{|c|}{$282(234)$} & & $.282(2.34)$ & & \\
\hline \multicolumn{7}{|l|}{15} \\
\hline $16 a$ & & & $.182(3.32)$ & $.182(3.32)$ & & \\
\hline $16 \mathrm{~b}$ & & & $.207(4.04)$ & $.207(4.04)$ & & \\
\hline \multicolumn{7}{|l|}{$16 c$} \\
\hline \multirow{2}{*}{\multicolumn{7}{|c|}{$\frac{71}{18}$}} \\
\hline & & & & & & \\
\hline 19 & & & $.198(3.40)$ & $.198(3.40)$ & & \\
\hline \multicolumn{7}{|l|}{20} \\
\hline \multicolumn{7}{|l|}{21} \\
\hline 22 & & & $.190(10.8)$ & $.190(10.8)$ & & \\
\hline \multicolumn{7}{|l|}{$23 a$} \\
\hline \multicolumn{7}{|c|}{$.415(2.07)$} \\
\hline \multirow{2}{*}{\multicolumn{7}{|c|}{25}} \\
\hline & & & & & & \\
\hline \multicolumn{7}{|c|}{$.592(2.60)$} \\
\hline$\frac{21}{28}$ & & & $.132(4.10)$ & $.132(4.10)$ & & \\
\hline 29 & & & & $.244(2.10)$ & & \\
\hline 30 & & & & & & \\
\hline
\end{tabular}

- positive coefficient

- negative coefficient

"voldo" - "age of the oldest vehicle involved is > 7 years" indicator variable

"maxpass" - "the largest number of occupants in all vehicles involved" quantitative variable

"age5y" - "age of the youngest driver is $\geq 50$ years" indicator variable 
Table C.5 (Continued)

\begin{tabular}{|c|c|c|c|c|c|c|}
\hline \multirow{3}{*}{ \# } & \multicolumn{6}{|c|}{ Coefficient (t-ratio) } \\
\hline & \multicolumn{2}{|c|}{$\mathrm{ff}\left[\mathrm{X}_{35}\right]$} & \multicolumn{2}{|c|}{$\mathrm{mm}\left[\mathrm{X}_{35}\right]$} & \multicolumn{2}{|c|}{$\mathrm{mf}\left[\mathrm{X}_{35}\right]$} \\
\hline & fatality & injury & fatality & injury & fatality & injury \\
\hline \multirow{2}{*}{\multicolumn{7}{|c|}{1}} \\
\hline & & & & & & \\
\hline \multicolumn{7}{|l|}{3} \\
\hline 4 & & & & $-.676(-3.68)$ & & \\
\hline \multicolumn{7}{|l|}{5} \\
\hline 6 & & & & & & \\
\hline \multicolumn{7}{|l|}{7} \\
\hline \multicolumn{7}{|l|}{$8 a$} \\
\hline \multicolumn{7}{|l|}{$\begin{array}{r}8 \boldsymbol{b} \\
9\end{array}$} \\
\hline 10 & $.448(2.66)$ & $.448(2.66)$ & & & & \\
\hline \multicolumn{7}{|l|}{11} \\
\hline \multicolumn{7}{|l|}{12} \\
\hline \multirow{2}{*}{\multicolumn{7}{|c|}{13}} \\
\hline \multirow{2}{*}{\multicolumn{7}{|c|}{$\begin{array}{l}\mathbf{1 4} \\
15\end{array}$}} \\
\hline & & & & & & \\
\hline $16 \mathrm{a}$ & & $.280(2.53)$ & & & & \\
\hline $16 \mathrm{~b}$ & & $.462(4.21)$ & & & & \\
\hline \multicolumn{7}{|l|}{$16 c$} \\
\hline \multirow{2}{*}{\multicolumn{6}{|c|}{$\frac{17}{18}$}} & \\
\hline & & & & & & \\
\hline 19 & & & $-.245(-2.27)$ & $-.245(-2.27)$ & & \\
\hline \multicolumn{7}{|l|}{20} \\
\hline \multicolumn{7}{|l|}{21} \\
\hline 22 & & & & $-.262(-7.73)$ & & \\
\hline $23 a$ & & & & & $.473(2.49)$ & $.473(2.49)$ \\
\hline \multirow{2}{*}{\multicolumn{7}{|c|}{$\frac{23 b}{24}$}} \\
\hline & & & & & & \\
\hline \multicolumn{7}{|c|}{25} \\
\hline \multicolumn{7}{|c|}{$-1.97(-2.50)$} \\
\hline \\
\hline 28 & & & $-.306(-3.73)$ & $-.306(-3.73)$ & & \\
\hline 29 & & & & & & \\
\hline 30 & & & & & & \\
\hline
\end{tabular}

- positive coefficient

- negative coefficient

"ff" - "two female drivers involved into a two-vehicle accident" indicator variable

"mm" - "two male drivers involved into a two-vehicle accident" indicator variable

"mf" - "male and female drivers involved into a two-vehicle accident" indicator variable 
Table C.6 Multinomial logit models for 2006 accident severity

\begin{tabular}{|c|c|c|c|c|c|c|c|c|}
\hline \multirow{3}{*}{ \# } & \multirow{3}{*}{\multicolumn{2}{|c|}{ 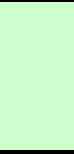 }} & \multirow{3}{*}{ Model name } & \multirow{2}{*}{\multicolumn{2}{|c|}{ Log-likelihood }} & \multirow{3}{*}{$R^{2}$} & \multirow{2}{*}{\multicolumn{2}{|c|}{$\begin{array}{c}\text { Coefficient (t-ratio) } \\
\mathrm{X}_{29}\end{array}$}} \\
\hline & & & & & & & & \\
\hline & & & & model & restricted* & & fatality $\left[\boldsymbol{\beta}_{1}\right]$ & injury $\left[\boldsymbol{\beta}_{2}\right]$ \\
\hline 1 & \multirow{8}{*}{ 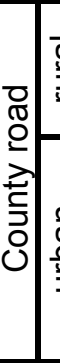 } & \multirow{3}{*}{$\frac{\sqrt[\pi]{2}}{2}$} & (car/SUV)+(car/SUV) & -2345.1 & -2457.6 & .046 & $.0396(5.48)$ & $.0396(5.48)$ \\
\hline 2 & & & (car/SUV)+(truck) & -113.99 & -135.50 & .159 & $.0648(3.06)$ & $.0648(3.06)$ \\
\hline 3 & & & one vehicle & -7152.1 & -8621.2 & .170 & $.00506(2.04)$ & $.00506(2.04$ \\
\hline $4 a$ & & & $($ car $)+($ car $)$ & -356.34 & -256.92 & -.387 & & \\
\hline $4 b$ & & \multirow{4}{*}{$\begin{array}{l}\frac{c}{\nwarrow} \\
\stackrel{9}{2}\end{array}$} & (car)+(SUV) & -232.26 & -106.65 & $\mathrm{n} / \mathrm{a}$ & & $.0613(2.43)$ \\
\hline $4 c$ & & & (SUV)+(SUV) & -183.12 & -100.57 & -.821 & & \\
\hline 5 & & & (car/SUV)+(truck) & -16.716 & -24.014 & .304 & & \\
\hline 6 & & & one vehicle & -367.48 & -418.24 & .121 & & \\
\hline 7 & \multirow{3}{*}{\multicolumn{2}{|c|}{ 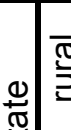 }} & (car/SUV)+(car/SUV) & -459.31 & -485.53 & .054 & & \\
\hline 8 & & & (car/SUV)+(truck) & -271.78 & -294.68 & .078 & & \\
\hline 9 & & & one vehicle & -1491.0 & -1718.3 & .132 & & \\
\hline 10 & $\frac{5}{0}$ & \multirow{3}{*}{\begin{tabular}{|l}
\multicolumn{2}{c}{} \\
$\frac{c}{2}$ \\
$\frac{0}{3}$ \\
\end{tabular}} & $($ (car/SUV)+(car/SUV) & -853.29 & -887.05 & .038 & & \\
\hline 11 & 全 & & (car/SUV)+(truck) & -252.55 & -278.96 & .097 & & \\
\hline 12 & & & one vehicle & -900.79 & -1032.2 & .127 & & \\
\hline 13 & \multirow{3}{*}{$\begin{array}{l}\frac{0}{5} \\
\end{array}$} & \multirow{3}{*}{ 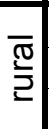 } & (car/SUV)+(car/SUV) & -560.40 & -605.43 & .074 & $.248(3.48)$ & $.0416(3.25)$ \\
\hline 14 & & & (car/SUV)+(truck) & -72.403 & -84.740 & .146 & $.127(2.50)$ & $.127(2.50)$ \\
\hline 15 & & & one vehicle & -3704.3 & -4873.2 & .240 & $.0636(2.34)$ & \\
\hline 16 & \multirow{3}{*}{ 竧 } & \multirow{3}{*}{\begin{tabular}{l|}
$\frac{0}{0}$ \\
$\frac{0}{5}$ \\
$\frac{1}{5}$
\end{tabular}} & (car/SUV)+(car/SUV) & -3225.9 & -3368.2 & .042 & $.252(3.35)$ & $.0290(7.95)$ \\
\hline 17 & & & (car/SUV)+(truck) & -192.97 & -203.31 & .051 & & \\
\hline 18 & & & one vehicle & -899.36 & -982.58 & .085 & & \\
\hline 19 & \multirow{3}{*}{\multicolumn{2}{|c|}{$\stackrel{\bar{\pi}}{\underline{2}}$}} & $($ (car/SUV)+(car/SUV) & -1068.42 & -1129.4 & .054 & $.0414(6.13)$ & $.0414(6.13)$ \\
\hline 20 & & & (car/SUV)+(truck) & -77.136 & -28.602 & $\mathrm{n} / \mathrm{a}$ & & \\
\hline 21 & & & one vehicle & -317.09 & -382.38 & .171 & & \\
\hline $22 a$ & \multirow{5}{*}{ 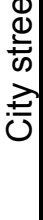 } & \multirow{5}{*}{$\begin{array}{l}\frac{c}{\mathbb{d}} \\
\stackrel{2}{y}\end{array}$} & (car)+(car) & -6828.1 & -7148.6 & .045 & $.0251(6.33)$ & $.0251(6.33)$ \\
\hline $22 b$ & & & (car)+(SUV) & -4289.5 & -4480.3 & .043 & $.0218(4.65)$ & $.0218(4.65)$ \\
\hline $22 c$ & & & (SUV)+(SUV) & -1341.6 & -1413.2 & .051 & $.0343(4.20)$ & $.0343(4.20)$ \\
\hline 23 & & & (car/SUV)+(truck) & -520.63 & -563.91 & .077 & $.0284(2.34)$ & $.0284(2.34)$ \\
\hline 24 & & & one vehicle & -2681.6 & -2955.4 & .093 & & \\
\hline 25 & & \multirow{3}{*}{ : } & $($ car/SUV)+(car/SUV) & -380.51 & -412.77 & .078 & & \\
\hline 26 & & & (car/SUV)+(truck) & -237.97 & -268.53 & .113 & $.0608(3.07)$ & $.0608(3.07)$ \\
\hline 27 & $\frac{0}{5}$ & & one vehicle & -1491.0 & -1917.7 & .223 & & \\
\hline 28 & \multirow{3}{*}{$\begin{array}{l}\text { 힌 } \\
\text { 心 } \\
\end{array}$} & \multirow{3}{*}{$\begin{array}{l}\frac{7}{\pi} \\
\frac{0}{3} \\
\frac{1}{3}\end{array}$} & (car/SUV)+(car/SUV) & -967.22 & -1003.9 & .037 & $.0154(2.14)$ & $.0154(2.14)$ \\
\hline 29 & & & (car/SUV)+(truck) & -185.87 & -209.82 & .114 & $.0586(3.60)$ & $.0586(3.60)$ \\
\hline 30 & & & one vehicle & -176.49 & -202.75 & .129 & & \\
\hline
\end{tabular}

- positive coefficient $\quad . .$. - negative coefficient

* - restricted log-likelihood found by setting all coefficients except intercepts to zero

** - models are estimated by using procedure A on page 32 , except the models marked by bold numbers and estimated by using procedure $B$ on page 33

$\mathrm{X}_{29}$ " - "posted speed limit (if the same for all vehicles involved)" quantitative variable 
Table C.6 (Continued)

\begin{tabular}{|c|c|c|c|c|c|c|}
\hline \multirow{2}{*}{$\#$} & \multicolumn{3}{|c|}{ Coefficient (t-ratio) } \\
\hline & \multicolumn{2}{|c|}{ constant } & \multicolumn{2}{c|}{ wint $\left[\mathrm{X}_{3}\right]$} & \multicolumn{2}{c|}{ sum $\left[\mathrm{X}_{3}\right]$} \\
\hline & fatality & injury & fatality & injury & fatality & injury \\
\hline 1 & $-9.88(-8.23)$ & $-3.45(-10.0)$ & & & & \\
\hline $\mathbf{2}$ & $-6.57(-4.36)$ & $-5.00(-4.82)$ & & & & \\
\hline 3 & $-5.31(-21.2)$ & $-2.25(-14.9)$ & & & & \\
\hline $\mathbf{4 a}$ & & $.954(5.64)$ & & & & \\
\hline $\mathbf{4 b}$ & & $-4.09(-3.78)$ & & & & \\
\hline $\mathbf{4 c}$ & & & & & & \\
\hline $\mathbf{5}$ & $-8.86(-4.10)$ & $-7.25(-3.69)$ & & & & \\
\hline 6 & $-7.00(-9.20)$ & $-2.54(-8.65)$ & & & & \\
\hline 7 & $-6.27(-11.2)$ & $-2.89(-10.2)$ & & & & \\
\hline $\mathbf{8}$ & $-5.01(-10.8)$ & $-3.24(-8.56)$ & & & & \\
\hline 9 & $-10.2(-7.26)$ & $-3.76(-22.9)$ & & & & \\
\hline 10 & $-8.42(-8.23)$ & $-2.90(-12.5)$ & & & & \\
\hline $\mathbf{1 1}$ & $-6.05(-8.32)$ & $-2.70(-10.9)$ & & & & \\
\hline 12 & $-6.62(-15.0)$ & $-3.84(-15.8)$ & & $-.414(-2.69)$ & & \\
\hline 13 & $-18.5(-4.74)$ & $-5.00(-5.88)$ & & & & \\
\hline $\mathbf{1 4}$ & $-9.24(-3.42)$ & $-8.38(-3.12)$ & & & & \\
\hline 15 & $-7.83(-5.28)$ & $-1.78(-16.9)$ & & & & \\
\hline 16 & $-19.1(-4.58)$ & $-2.77(-16.6)$ & & & & \\
\hline $\mathbf{1 7}$ & $-3.43(-3.37)$ & $-2.22(-12.6)$ & & & & \\
\hline 18 & $-4.93(-14.3)$ & $-1.99(-8.01)$ & & & & \\
\hline 19 & $-9.35(-9.03)$ & $-3.58(-11.8)$ & & & & \\
\hline $\mathbf{2 0}$ & & $-2.08(-4.04)$ & & & & \\
\hline 21 & $-4.65(-8.25)$ & $-1.07(-3.87)$ & & & & \\
\hline $22 a$ & $-9.47(-13.1)$ & $-2.58(-15.9)$ & & & & \\
\hline $22 b$ & $-8.25(-15.5)$ & $-2.27(-11.9)$ & & & & \\
\hline $22 c$ & $-8.34(-12.6)$ & $-3.04(-9.33)$ & & & & \\
\hline 23 & $-8.69(-7.93)$ & $-4.19(-8.75)$ & & & & \\
\hline 24 & $-5.63(-21.9)$ & $-3.42(-15.1)$ & & & & \\
\hline 25 & $-4.44(-9.52)$ & $-2.04(4.11)$ & & & & \\
\hline $\mathbf{2 6}$ & $-7.48(-6.35)$ & $-4.32(-3.99)$ & & & & \\
\hline 27 & $-4.93(-10.5)$ & $-1.24(-3.40)$ & & & & \\
\hline 28 & $-8.27(-7.86)$ & $-2.13(-6.45)$ & & & & \\
\hline $\mathbf{2 9}$ & $-11.4(-4.75)$ & $-3.56(-5.04)$ & & & & \\
\hline 30 & $-5.39(-7.12)$ & $-2.32(-4.48)$ & & & & \\
\hline
\end{tabular}

- positive coefficient

- negative coefficient

"constant" - "constant term (intercept)" quantitative variable

"wint" - - "winter season" indicator variable

"sum" _ _ - "summer season" indicator variable 
Table C.6 (Continued)

\begin{tabular}{|c|c|c|c|c|c|c|}
\hline \multirow{3}{*}{ \# } & \multicolumn{6}{|c|}{ Coefficient (t-ratio) } \\
\hline & \multicolumn{2}{|c|}{ fall $\left[X_{3}\right]$} & \multicolumn{2}{|c|}{$\operatorname{mon}\left[\mathrm{X}_{4}\right]$} & \multicolumn{2}{|c|}{ sund $\left[\mathrm{X}_{4}\right]$} \\
\hline & fatality & injury & fatality & injury & fatality & injury \\
\hline 1 & $2.28(4.01)$ & & & & & \\
\hline \multirow{2}{*}{\multicolumn{7}{|c|}{$\frac{2}{3}$}} \\
\hline & & & & & & \\
\hline \multicolumn{7}{|l|}{$4 a$} \\
\hline \multirow{2}{*}{\multicolumn{7}{|c|}{$\frac{4 b}{4 c}$}} \\
\hline & & & & & & \\
\hline \multicolumn{7}{|c|}{$3.49(2.53)$} \\
\hline \multicolumn{7}{|c|}{$1-1$} \\
\hline \multicolumn{7}{|l|}{7} \\
\hline \multirow{2}{*}{\multicolumn{7}{|c|}{$\frac{8}{9}$}} \\
\hline & & & & & & \\
\hline \multicolumn{7}{|l|}{10} \\
\hline \multirow{2}{*}{\multicolumn{7}{|c|}{$-1.18(-3.64)$}} \\
\hline & & & & & & \\
\hline \multirow{2}{*}{\multicolumn{7}{|c|}{13}} \\
\hline 14 & & & & & & \\
\hline \multicolumn{7}{|l|}{15} \\
\hline \multicolumn{7}{|l|}{16} \\
\hline \multirow{2}{*}{\multicolumn{7}{|c|}{$\frac{17}{18}$}} \\
\hline 18 & & & & & & \\
\hline \multicolumn{7}{|l|}{19} \\
\hline \multirow{2}{*}{\multicolumn{7}{|c|}{$\frac{20}{21}$}} \\
\hline & & & & & & \\
\hline \multicolumn{7}{|l|}{$22 \mathrm{a}$} \\
\hline \multirow{2}{*}{\multicolumn{7}{|c|}{$1.79(2.52)$}} \\
\hline & & & & & & \\
\hline \multicolumn{7}{|c|}{$.876(2.19)$} \\
\hline 24 & & & & & & \\
\hline \multicolumn{3}{|c|}{$2.14(4.11)$} & & & & \\
\hline \multirow{2}{*}{\multicolumn{7}{|c|}{$-1.49(-3.92)$}} \\
\hline & & & & & & \\
\hline \multicolumn{7}{|l|}{28} \\
\hline 29 & $-.714(-2.00)$ & $-.714(-2.00)$ & & & & \\
\hline 30 & & & & & & \\
\hline
\end{tabular}

- positive coefficient

- negative coefficient

"fall" _ - "fall season" indicator variable

"mon" - "Monday" indicator variable

"sund" - "Sunday" indicator variable 
Table C.6 (Continued)

\begin{tabular}{|c|c|c|c|c|c|c|}
\hline \multirow{3}{*}{ \# } & \multicolumn{6}{|c|}{ Coefficient (t-ratio) } \\
\hline & \multicolumn{2}{|c|}{ wday $\left[\mathrm{X}_{4}\right]$} & \multicolumn{2}{|c|}{ wed $\left[\mathrm{X}_{4}\right]$} & \multicolumn{2}{|c|}{ jobend $\left[X_{5}\right]$} \\
\hline & fatality & injury & fatality & injury & fatality & injury \\
\hline \multirow{2}{*}{\multicolumn{7}{|c|}{1}} \\
\hline & & & & & & \\
\hline 3 & $-.159(-3.41)$ & $-.159(-3.41)$ & & & & \\
\hline \multicolumn{7}{|l|}{$4 a$} \\
\hline \multirow{2}{*}{\multicolumn{7}{|c|}{$4 b$}} \\
\hline \multirow{2}{*}{\multicolumn{7}{|c|}{$\frac{46}{5}$}} \\
\hline & & & & & & \\
\hline \multicolumn{7}{|l|}{6} \\
\hline \multirow{2}{*}{\multicolumn{7}{|c|}{$\frac{7}{8}$}} \\
\hline & & & & & & \\
\hline \multicolumn{7}{|l|}{9} \\
\hline \multicolumn{7}{|l|}{10} \\
\hline \multirow{2}{*}{\multicolumn{7}{|c|}{11}} \\
\hline 12 & & & & & & \\
\hline \multicolumn{7}{|l|}{13} \\
\hline \multicolumn{7}{|l|}{14} \\
\hline \multirow{2}{*}{\multicolumn{7}{|c|}{$\frac{15}{116}$}} \\
\hline \multicolumn{6}{|l|}{16} & \\
\hline 17 & $-2.81(-1.97)$ & & & & & \\
\hline \multicolumn{7}{|c|}{ ) } \\
\hline \multicolumn{7}{|l|}{19} \\
\hline \multirow{2}{*}{\multicolumn{7}{|c|}{$\frac{20}{21}$}} \\
\hline & & & & & & \\
\hline \multicolumn{7}{|c|}{ 22a } \\
\hline \multirow{2}{*}{\multicolumn{7}{|c|}{$22 \mathrm{c}$}} \\
\hline & & & & & & \\
\hline \multicolumn{7}{|l|}{23} \\
\hline 24 & & & & $-.264(-2.58)$ & $.214(2.45)$ & $.214(2.45)$ \\
\hline \multicolumn{7}{|l|}{25} \\
\hline \multirow{2}{*}{\multicolumn{7}{|c|}{$\begin{array}{l}26 \\
27\end{array}$}} \\
\hline \multirow{2}{*}{\multicolumn{7}{|c|}{28}} \\
\hline & & & & & & \\
\hline 29 & & & & & & \\
\hline 30 & & & & & & \\
\hline
\end{tabular}

- positive coefficient

- negative coefficient

"wday" - - "any weekday except Saturday and Sunday" indicator variable

"wed" _ - "Wednesday" indicator variable

"jobend" - "end of job hours: from 16:00 to 19:00" indicator variable 
Table C.6 (Continued)

\begin{tabular}{|c|c|c|c|c|c|c|}
\hline \multirow{3}{*}{ \# } & \multicolumn{6}{|c|}{ Coefficient (t-ratio) } \\
\hline & \multicolumn{2}{|c|}{ peak $\left[X_{5}\right]$} & \multicolumn{2}{|c|}{ nigh $\left[X_{5}\right]$} & \multicolumn{2}{|c|}{ dayt $\left[X_{5}\right]$} \\
\hline & fatality & injury & fatality & injury & fatality & injury \\
\hline 1 & & & $2.15(2.32)$ & & & \\
\hline \multicolumn{7}{|l|}{2} \\
\hline 3 & & & & & & \\
\hline \multicolumn{7}{|l|}{$4 a$} \\
\hline \multicolumn{7}{|l|}{$4 b$} \\
\hline \multirow{2}{*}{\multicolumn{7}{|c|}{$\frac{4 c}{5}$}} \\
\hline & & & & & & \\
\hline \multicolumn{7}{|l|}{6} \\
\hline 7 & & & $1.06(2.27)$ & $1.06(2.27)$ & & \\
\hline 8 & & & & & $-.641(-2.62)$ & $-.641(-2.62)$ \\
\hline 9 & & & & & & $.252(2.46)$ \\
\hline \multicolumn{7}{|l|}{10} \\
\hline \multirow{2}{*}{\multicolumn{7}{|c|}{$\begin{array}{l}11 \\
12\end{array}$}} \\
\hline & & & & & & \\
\hline \multicolumn{7}{|l|}{13} \\
\hline \multicolumn{7}{|l|}{14} \\
\hline 15 & & & & & & $.178(2.59)$ \\
\hline \multicolumn{7}{|l|}{16} \\
\hline \multirow{2}{*}{\multicolumn{7}{|c|}{17}} \\
\hline & & & & & & \\
\hline \multicolumn{7}{|l|}{19} \\
\hline \multicolumn{7}{|l|}{20} \\
\hline 21 & & & & & & $.477(2.11)$ \\
\hline \multirow{2}{*}{\multicolumn{7}{|c|}{$\frac{-.117(-2.39)}{-.137(-2.20)}$}} \\
\hline & & $-.137(-2.20)$ & & & & \\
\hline \multicolumn{7}{|l|}{$22 \mathrm{c}$} \\
\hline \multicolumn{7}{|l|}{$\begin{array}{l}23 \\
24\end{array}$} \\
\hline \multicolumn{7}{|l|}{25} \\
\hline \multicolumn{7}{|l|}{26} \\
\hline \multicolumn{7}{|l|}{27} \\
\hline \multicolumn{7}{|c|}{\begin{tabular}{l|l}
28 \\
0
\end{tabular}} \\
\hline 29 & & & & & & \\
\hline 30 & & & & & & \\
\hline
\end{tabular}

- positive coefficient

- negative coefficient

"peak" - "rush hours: 7:00 to 9:00 OR 17:00 to 19:00" indicator variable

"nigh" - "late night hours: from 1:00 to 5:00" indicator variable

"dayt" - "day hours: from 9:00 to 17:00" indicator variable 
Table C.6 (Continued)

\begin{tabular}{|c|c|c|c|c|c|c|}
\hline \multirow{3}{*}{ \# } & \multicolumn{6}{|c|}{ Coefficient (t-ratio) } \\
\hline & \multicolumn{2}{|c|}{ lunch $\left[X_{5}\right]$} & \multicolumn{2}{|c|}{ even $\left[X_{5}\right]$} & \multicolumn{2}{|c|}{ nocons $\left[\mathrm{X}_{13}\right]$} \\
\hline & fatality & injury & fatality & injury & fatality & injury \\
\hline \multicolumn{7}{|l|}{1} \\
\hline 2 & $-1.25(-2.44)$ & $-1.25(-2.44)$ & & & & \\
\hline \multicolumn{7}{|c|}{ 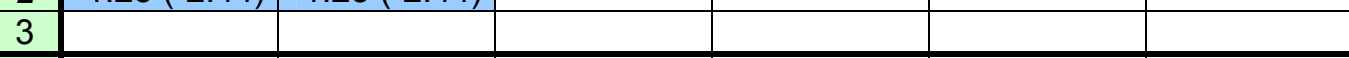 } \\
\hline \multicolumn{7}{|l|}{$4 a$} \\
\hline \multicolumn{7}{|l|}{$4 b$} \\
\hline $4 c$ & & & $-3.10(-5.13)$ & $-3.10(-5.13)$ & & \\
\hline \multicolumn{7}{|c|}{$6,1,1, \ldots}$, \\
\hline \multicolumn{7}{|l|}{6} \\
\hline \multirow{2}{*}{\multicolumn{7}{|c|}{$\begin{array}{l}7 \\
8\end{array}$}} \\
\hline \multirow{2}{*}{\multicolumn{7}{|c|}{8}} \\
\hline & & & & & & \\
\hline$\overline{10}$ & $.581(3.23)$ & $.581(3.23)$ & & & & \\
\hline \multicolumn{7}{|l|}{11} \\
\hline 12 & & & & & & \\
\hline \multicolumn{7}{|l|}{13} \\
\hline \multicolumn{7}{|l|}{14} \\
\hline \multicolumn{7}{|l|}{15} \\
\hline \multirow{2}{*}{\multicolumn{7}{|c|}{$\frac{16}{17}$}} \\
\hline & & & & & & \\
\hline \multicolumn{7}{|l|}{18} \\
\hline \multicolumn{7}{|l|}{19} \\
\hline \multirow{2}{*}{\multicolumn{7}{|c|}{$\frac{20}{21}$}} \\
\hline & & & & & & \\
\hline \multicolumn{7}{|c|}{$22 \mathrm{a}$} \\
\hline \multicolumn{7}{|c|}{$22 \mathrm{~b}$} \\
\hline \multirow{2}{*}{\multicolumn{7}{|c|}{23}} \\
\hline & & & & & & \\
\hline \multicolumn{7}{|c|}{24} \\
\hline \multicolumn{7}{|c|}{25} \\
\hline \multicolumn{7}{|l|}{26} \\
\hline 27 & & & & & $-.677(-2.06)$ & $-.677(-2.06)$ \\
\hline 28 & & & & & & \\
\hline 29 & & & & & & \\
\hline 30 & & & & & & \\
\hline
\end{tabular}

.... - positive coefficient

- negative coefficient

"lunch" - "lunch hours: 11:00 to 14:00" indicator variable

"even" - "evening hours: 17:00 to 22:00" indicator variable

"nocons" - "no construction at the accident location" indicator variable 
Table C.6 (Continued)

\begin{tabular}{|c|c|c|c|c|c|c|}
\hline \multirow{3}{*}{ \# } & \multicolumn{6}{|c|}{ Coefficient (t-ratio) } \\
\hline & \multicolumn{2}{|c|}{ cons $\left[\mathrm{X}_{13}\right]$} & \multicolumn{2}{|c|}{ dark $\left[\mathrm{X}_{14}\right]$} & \multicolumn{2}{|c|}{ day $\left[X_{14}\right]$} \\
\hline & fatality & injury & fatality & injury & fatality & injury \\
\hline \multirow{2}{*}{\multicolumn{7}{|c|}{$\frac{1}{2}$}} \\
\hline & & & & & & \\
\hline 3 & & & & & $-.738(-4.06)$ & \\
\hline \multicolumn{7}{|l|}{$4 a$} \\
\hline \multirow{2}{*}{\multicolumn{7}{|c|}{$\frac{4 b}{4 c}$}} \\
\hline & & & & & & \\
\hline \multicolumn{7}{|l|}{5} \\
\hline \multicolumn{7}{|l|}{6} \\
\hline 7 & & & $.482(2.12)$ & $.482(2.12)$ & & \\
\hline \multicolumn{7}{|l|}{8} \\
\hline \multicolumn{7}{|l|}{9} \\
\hline 10 & & & & & $-.425(-2.95)$ & $-.425(-2.95)$ \\
\hline \multicolumn{7}{|l|}{11} \\
\hline 12 & & & $1.40(2.33)$ & $1.40(2.33)$ & & \\
\hline 13 & & & $1.24(2.85)$ & & & \\
\hline \multicolumn{7}{|l|}{14} \\
\hline 15 & & & & & $-.931(-3.80)$ & \\
\hline \multicolumn{7}{|l|}{16} \\
\hline 17 & & & & $1.52(3.16)$ & & \\
\hline 18 & & & & & $.529(4.35)$ & $.529(4.35)$ \\
\hline 19 & $-1.51(-2.06)$ & $-1.51(-2.06)$ & & & & \\
\hline \multicolumn{7}{|c|}{\begin{tabular}{|l|l|}
20 & \\
\end{tabular}} \\
\hline \multicolumn{7}{|c|}{21} \\
\hline $22 \mathrm{a}$ & & & & & & \\
\hline \multicolumn{7}{|c|}{$-.150(-2.26)$} \\
\hline \multicolumn{7}{|c|}{$\frac{22 c}{23}$} \\
\hline$\frac{23}{24}$ & \multicolumn{5}{|c|}{23} & \\
\hline \multicolumn{7}{|l|}{25} \\
\hline \multicolumn{7}{|l|}{26} \\
\hline 27 & & & & & $.235(2.33)$ & $.235(2.33)$ \\
\hline \multicolumn{7}{|l|}{28} \\
\hline 29 & & & & & & \\
\hline 30 & & & & & & \\
\hline
\end{tabular}

- positive coefficient

- negative coefficient

"cons" - - "construction at the accident location" indicator variable

"dark" - - "dark time with no street lights" indicator variable

"day" _ - "daylight time" indicator variable 
Table C.6 (Continued)

\begin{tabular}{|c|c|c|c|c|c|c|}
\hline \multirow{3}{*}{ \# } & \multicolumn{6}{|c|}{ Coefficient (t-ratio) } \\
\hline & \multicolumn{2}{|c|}{ dawn $\left[\mathrm{X}_{14}\right]$} & \multicolumn{2}{|c|}{ darklamp $\left[\mathrm{X}_{14}\right]$} & \multicolumn{2}{|c|}{ precip $\left[\mathrm{X}_{15}\right]$} \\
\hline & fatality & injury & fatality & injury & fatality & injury \\
\hline \multicolumn{7}{|l|}{$\frac{1}{2}$} \\
\hline \multirow{2}{*}{\multicolumn{7}{|c|}{$\frac{2}{3}$}} \\
\hline & & & & & & \\
\hline \multicolumn{7}{|l|}{$4 a$} \\
\hline \multicolumn{7}{|l|}{$4 b$} \\
\hline $4 c$ & \\
\hline \multirow{2}{*}{\multicolumn{7}{|c|}{6}} \\
\hline & & & & & & \\
\hline \multicolumn{7}{|l|}{7} \\
\hline \multirow{2}{*}{\multicolumn{7}{|c|}{$\frac{8}{9}$}} \\
\hline 9 & & & & & & \\
\hline \multicolumn{7}{|l|}{10} \\
\hline \multirow{2}{*}{\multicolumn{7}{|c|}{$.722(2.45)$}} \\
\hline & & & & & & \\
\hline \multicolumn{7}{|l|}{13} \\
\hline 14 & & & $2.97(2.42)$ & $2.97(2.42)$ & & \\
\hline 15 & & & & & $-1.09(-2.56)$ & \\
\hline \multicolumn{7}{|l|}{16} \\
\hline \multirow{2}{*}{\multicolumn{7}{|c|}{$\frac{17}{10}$}} \\
\hline 18 & & & & & & \\
\hline \multicolumn{7}{|l|}{$\frac{10}{19}$} \\
\hline \multicolumn{7}{|c|}{20} \\
\hline \multicolumn{7}{|c|}{21} \\
\hline \multirow{2}{*}{\multicolumn{7}{|c|}{$\frac{22 a}{22 b}$}} \\
\hline & & & & & & \\
\hline \multicolumn{7}{|c|}{$22 \mathrm{c}$} \\
\hline \multicolumn{7}{|c|}{$3.14(2.21)$} \\
\hline \multirow{2}{*}{\multicolumn{7}{|c|}{$\frac{24}{25}$}} \\
\hline & & & & & & \\
\hline \multicolumn{7}{|l|}{26} \\
\hline \multicolumn{7}{|c|}{27} \\
\hline 28 & & & & & & \\
\hline 29 & & & & & & \\
\hline 30 & & & & & & \\
\hline
\end{tabular}

- positive coefficient

- negative coefficient

\footnotetext{
"dawn" _ - "dawn OR dask" indicator variable

"darklamp" - "dark AND street lights on" indicator variable

"precip" - " "precipitation: rain OR snow OR sleet OR hail OR freezing rain" indicator variable
} 
Table C.6 (Continued)

\begin{tabular}{|c|c|c|c|c|c|c|}
\hline \multirow{3}{*}{ \# } & \multicolumn{6}{|c|}{ Coefficient (t-ratio) } \\
\hline & \multicolumn{2}{|c|}{ snow $\left[\mathrm{X}_{15}\right]$} & \multicolumn{2}{|c|}{ clear $\left[\mathrm{X}_{15}\right]$} & \multicolumn{2}{|c|}{$\operatorname{dry}\left[\mathrm{X}_{16}\right]$} \\
\hline & fatality & injury & fatality & injury & fatality & injury \\
\hline \multirow{2}{*}{\multicolumn{7}{|c|}{$\frac{1}{2}$}} \\
\hline & & & & & & \\
\hline \multicolumn{7}{|c|}{$.0929(2.11)$} \\
\hline \multicolumn{7}{|l|}{$4 a$} \\
\hline \multicolumn{7}{|l|}{$\frac{4 b}{4 c}$} \\
\hline $4 c$ & & & & & & \\
\hline \multicolumn{7}{|l|}{5} \\
\hline \multicolumn{7}{|l|}{6} \\
\hline \multirow{2}{*}{\multicolumn{7}{|c|}{$\frac{7}{8}$}} \\
\hline & & & & & & \\
\hline \multicolumn{7}{|l|}{9} \\
\hline \multirow{2}{*}{\multicolumn{7}{|c|}{$\frac{10}{11}$}} \\
\hline & & & & & & \\
\hline \multicolumn{5}{|c|}{$.574(4.44)$} & $.574(4.44)$ & $.574(4.44)$ \\
\hline \multirow{2}{*}{\multicolumn{7}{|c|}{$\begin{array}{l}13 \\
14\end{array}$}} \\
\hline & & & & & & \\
\hline \multicolumn{7}{|l|}{15} \\
\hline \multicolumn{7}{|l|}{16} \\
\hline \multirow{2}{*}{\multicolumn{7}{|c|}{$\frac{17}{18}$}} \\
\hline & & & & & & \\
\hline \multicolumn{7}{|l|}{19} \\
\hline \multicolumn{7}{|l|}{20} \\
\hline 21 & & & $.518(2.46)$ & $.518(2.46)$ & & \\
\hline $22 \mathrm{a}$ & & & & $-.143(-3.36)$ & & \\
\hline \multicolumn{7}{|c|}{$22 \mathrm{~b}$} \\
\hline $22 \mathrm{c}$ & & & & & & \\
\hline \multicolumn{7}{|c|}{23} \\
\hline 24 & $-.651(-3.07)$ & $-.651(-3.07)$ & & & $.385(4.78)$ & $.385(4.78)$ \\
\hline \multicolumn{7}{|c|}{\begin{tabular}{|l|l|}
25 & \\
\end{tabular}} \\
\hline \multirow{2}{*}{\multicolumn{7}{|c|}{26}} \\
\hline & & & & & & \\
\hline \multicolumn{7}{|c|}{28} \\
\hline 29 & & & & & & \\
\hline 30 & & & & & & \\
\hline
\end{tabular}

.... - positive coefficient

.... - negative coefficient

"snow" - "snowing weather" indicator variable

"clear" - "clear weather" indicator variable

"dry" _ - "roadway surface is dry" indicator variable 
Table C.6 (Continued)

\begin{tabular}{|c|c|c|c|c|c|c|}
\hline \multirow{3}{*}{ \# } & \multicolumn{6}{|c|}{ Coefficient (t-ratio) } \\
\hline & \multicolumn{2}{|c|}{ wet $\left[\mathrm{X}_{16}\right]$} & \multicolumn{2}{|c|}{ ice $\left[\mathrm{X}_{16}\right]$} & \multicolumn{2}{|c|}{ lose $\left[X_{16}\right]$} \\
\hline & fatality & injury & fatality & injury & fatality & injury \\
\hline \multirow{2}{*}{\multicolumn{7}{|c|}{1}} \\
\hline & & & & & & \\
\hline 3 & & & & & $.428(3.30)$ & $.428(3.30)$ \\
\hline \multicolumn{7}{|l|}{$4 a$} \\
\hline \multirow{2}{*}{\multicolumn{7}{|c|}{$4 c$}} \\
\hline & & & & & & \\
\hline \multicolumn{7}{|l|}{5} \\
\hline \multirow{2}{*}{\multicolumn{7}{|c|}{$\frac{6}{7}$}} \\
\hline & & & & & & \\
\hline \multicolumn{7}{|l|}{8} \\
\hline \multicolumn{7}{|l|}{9} \\
\hline \multicolumn{7}{|l|}{10} \\
\hline \multirow{2}{*}{\multicolumn{7}{|c|}{$\begin{array}{l}11 \\
12\end{array}$}} \\
\hline & & & & & & \\
\hline \multicolumn{7}{|l|}{13} \\
\hline \multirow{2}{*}{\multicolumn{7}{|c|}{$\frac{14}{15}$}} \\
\hline & & & & & & \\
\hline \multicolumn{7}{|l|}{16} \\
\hline \multirow{2}{*}{\multicolumn{7}{|c|}{$\frac{17}{18}$}} \\
\hline & & & & & & \\
\hline \multicolumn{7}{|l|}{19} \\
\hline \multicolumn{7}{|l|}{20} \\
\hline 21 & & & $-2.16(-2.10)$ & $-2.16(-2.10)$ & & \\
\hline \multicolumn{7}{|c|}{$22 \mathrm{a}$} \\
\hline \multirow{2}{*}{\multicolumn{7}{|c|}{$\begin{array}{ll}22 b \\
22 c\end{array}$}} \\
\hline & & & & & & \\
\hline \multirow{2}{*}{\multicolumn{7}{|c|}{$.584(3.01)$}} \\
\hline & & & & & & \\
\hline \multicolumn{7}{|l|}{25} \\
\hline \multirow{2}{*}{\multicolumn{7}{|c|}{27}} \\
\hline & & & & & & \\
\hline \multicolumn{7}{|l|}{28} \\
\hline 29 & & & & & & \\
\hline 30 & & & & & & \\
\hline
\end{tabular}

- positive coefficient

- negative coefficient

"wet" - "roadway surface is wet" indicator variable

"ice" - "roadway surface is icy" indicator variable

"lose" - "roadway surface has loose material on it" indicator variable 
Table C.6 (Continued)

\begin{tabular}{|c|c|c|c|c|c|c|}
\hline \multirow{3}{*}{ \# } & \multicolumn{6}{|c|}{ Coefficient (t-ratio) } \\
\hline & \multicolumn{2}{|c|}{ water $\left[\mathrm{X}_{16}\right]$} & \multicolumn{2}{|c|}{ driv $\left[\mathrm{X}_{17}\right]$} & \multicolumn{2}{|c|}{ wall $\left[\mathrm{X}_{17}\right]$} \\
\hline & fatality & injury & fatality & injury & fatality & injury \\
\hline 1 & & & & & & \\
\hline \multicolumn{2}{|l|}{2} & & & & & \\
\hline \multicolumn{7}{|l|}{3} \\
\hline \multicolumn{7}{|l|}{$4 a$} \\
\hline \multicolumn{7}{|l|}{$4 b$} \\
\hline $4 c$ & & & $-1.13(-2.41)$ & & & \\
\hline \multicolumn{7}{|c|}{ …ㄴ. } \\
\hline \multicolumn{7}{|l|}{6} \\
\hline 7 & & & $.467(2.55)$ & $.467(2.55)$ & & \\
\hline \multicolumn{7}{|c|}{ 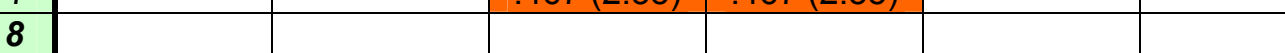 } \\
\hline \multicolumn{7}{|l|}{9} \\
\hline 10 & & & $.439(2.44)$ & $439(2.44)$ & & \\
\hline \multirow{2}{*}{\multicolumn{7}{|c|}{$2.30(2.40)$}} \\
\hline & & & & & & \\
\hline \multicolumn{7}{|l|}{13} \\
\hline \multicolumn{7}{|c|}{14} \\
\hline \multicolumn{7}{|l|}{15} \\
\hline \multicolumn{7}{|c|}{\begin{tabular}{|l|l|}
16 \\
17
\end{tabular}} \\
\hline 17 & & & & & & \\
\hline \multicolumn{7}{|l|}{18} \\
\hline \multicolumn{7}{|l|}{19} \\
\hline \multirow{2}{*}{\multicolumn{7}{|c|}{$\frac{20}{21}$}} \\
\hline & & & & & & \\
\hline \multicolumn{7}{|c|}{$22 \mathrm{a}$} \\
\hline \multirow{2}{*}{\multicolumn{7}{|c|}{$22 \mathrm{~b}$}} \\
\hline $22 c$ & & & & & & \\
\hline \multicolumn{7}{|c|}{23} \\
\hline 24 & & & $.281(3.83)$ & $.281(3.83)$ & & \\
\hline 25 & & & & & & \\
\hline 26 & & & & & $-2.10(-1.97)$ & \\
\hline 27 & & & & & & \\
\hline 28 & & & & & & \\
\hline 29 & & & & & & \\
\hline 30 & & & & & & \\
\hline
\end{tabular}

- positive coefficient

- negative coefficient

"water" - "roadway surface has water on it" indicator variable

"driv" - - "road median is a drivable" indicator variable

"wall" _ _ " "road median is a wall" indicator variable 
Table C.6 (Continued)

\begin{tabular}{|c|c|c|c|c|c|c|}
\hline \multirow{3}{*}{ \# } & \multicolumn{6}{|c|}{ Coefficient (t-ratio) } \\
\hline & \multicolumn{2}{|c|}{ nomed $\left[\mathrm{X}_{17}\right]$} & \multicolumn{2}{|c|}{ curb $\left[\mathrm{X}_{17}\right]$} & \multicolumn{2}{|c|}{ nojun $\left[\mathrm{X}_{18}\right]$} \\
\hline & fatality & injury & fatality & injury & fatality & injury \\
\hline \multicolumn{7}{|l|}{1} \\
\hline 2 & $-2.73(-2.10)$ & & & & & \\
\hline 3 & & & $-1.71(-2.57)$ & & $.167(2.35)$ & $.167(2.35)$ \\
\hline \multicolumn{7}{|l|}{$4 a$} \\
\hline \multirow{2}{*}{\multicolumn{7}{|c|}{$\begin{array}{l}4 b \\
4 c\end{array}$}} \\
\hline & & & & & & \\
\hline \multicolumn{7}{|l|}{5} \\
\hline \multicolumn{7}{|l|}{6} \\
\hline 7 & & & & & $.591(2.19)$ & $.591(2.19)$ \\
\hline \multicolumn{7}{|l|}{8} \\
\hline \multicolumn{7}{|l|}{9} \\
\hline 10 & & & & & $.536(3.73)$ & $.536(3.73)$ \\
\hline \multicolumn{7}{|l|}{11} \\
\hline 12 & & & \\
\hline 13 & & & & & & \\
\hline \multicolumn{7}{|l|}{14} \\
\hline \multicolumn{7}{|l|}{15} \\
\hline 16 & & & & & $-.367(-5.86)$ & $-.367(-5.86)$ \\
\hline \multicolumn{7}{|l|}{17} \\
\hline 18 & & & & & $-.548(-4.14)$ & $-.548(-4.14)$ \\
\hline 19 & & $-.236(-2.13)$ & & & & \\
\hline \multicolumn{7}{|l|}{20} \\
\hline \multicolumn{7}{|c|}{21} \\
\hline $22 \mathrm{a}$ & & $-.112(-2.37)$ & & & $-.389(-8.68)$ & $-.389(-8.68)$ \\
\hline $22 \mathrm{~b}$ & & & & & $-.252(-4.18)$ & $-.252(-4.18)$ \\
\hline \multicolumn{7}{|l|}{$22 \mathrm{c}$} \\
\hline \multicolumn{7}{|l|}{$\begin{array}{l}23 \\
24\end{array}$} \\
\hline 24 & & & & & & \\
\hline 25 & & & $-1.21(-2.38)$ & $-1.21(-2.38)$ & & \\
\hline \multirow{2}{*}{\multicolumn{7}{|c|}{$\begin{array}{l}26 \\
27\end{array}$}} \\
\hline \multirow{2}{*}{\multicolumn{7}{|c|}{28}} \\
\hline & & & & & & \\
\hline 29 & & & & & & \\
\hline 30 & & & & & & \\
\hline
\end{tabular}

$$
\begin{aligned}
& \ldots . \text { - positive coefficient } \\
& \ldots . \text { - negative coefficient }
\end{aligned}
$$

"nomed" - "no median" indicator variable

"curb" _ - "road median is curbed" indicator variable

"nojun" - " "no road junction at the accident location" indicator variable 
Table C.6 (Continued)

\begin{tabular}{|c|c|c|c|c|c|c|}
\hline \multirow{3}{*}{ \# } & \multicolumn{6}{|c|}{ Coefficient (t-ratio) } \\
\hline & \multicolumn{2}{|c|}{ way4 $\left[\mathrm{X}_{18}\right]$} & \multicolumn{2}{|c|}{$\mathrm{T}\left[\mathrm{X}_{18}\right]$} & \multicolumn{2}{|c|}{$\operatorname{str}\left[\mathrm{X}_{19}\right]$} \\
\hline & fatality & injury & fatality & injury & fatality & injury \\
\hline \multicolumn{7}{|l|}{1} \\
\hline 2 & $1.13(3.18)$ & $1.13(3.18)$ & & & & \\
\hline 3 & & & & & $-.194(-3.95)$ & $-.194(-3.95)$ \\
\hline \multicolumn{7}{|l|}{$4 a$} \\
\hline $4 b$ & & & & & & \\
\hline \multicolumn{7}{|l|}{$4 c$} \\
\hline \multicolumn{7}{|l|}{5} \\
\hline \multicolumn{7}{|l|}{6} \\
\hline \multicolumn{7}{|l|}{7} \\
\hline \multirow{2}{*}{\multicolumn{7}{|c|}{$3.31(2.65)$}} \\
\hline & & & & & & \\
\hline \multicolumn{7}{|l|}{10} \\
\hline \multirow{2}{*}{\multicolumn{7}{|c|}{$\begin{array}{l}11 \\
12\end{array}$}} \\
\hline & & & & & & \\
\hline \multirow{2}{*}{\multicolumn{7}{|c|}{13}} \\
\hline \multicolumn{2}{|l|}{14} & & & & & \\
\hline 15 & & & & & $-.322(-4.80)$ & $-.322(-4.80)$ \\
\hline \multicolumn{7}{|l|}{16} \\
\hline \multicolumn{7}{|l|}{17} \\
\hline \multicolumn{7}{|l|}{18} \\
\hline 19 & $.328(2.95)$ & $.328(2.95)$ & & & & \\
\hline \multicolumn{7}{|l|}{20} \\
\hline 21 & & & & & & \\
\hline \multicolumn{7}{|l|}{$22 \mathrm{a}$} \\
\hline \multirow{2}{*}{\multicolumn{7}{|c|}{$\begin{array}{l}22 b \\
22 c\end{array}$}} \\
\hline & & & & & & \\
\hline \multicolumn{6}{|c|}{$.682(4.11)$} & \\
\hline \multicolumn{7}{|l|}{24} \\
\hline \multirow{2}{*}{\multicolumn{7}{|c|}{.436 (2.03) }} \\
\hline \multirow{2}{*}{\multicolumn{7}{|c|}{$-.227(-2.02)$}} \\
\hline & & & & & $-.227(-2.02)$ & $-.227(-2.02)$ \\
\hline \multirow{2}{*}{\multicolumn{7}{|c|}{$\frac{28}{29}$}} \\
\hline & & & & & & \\
\hline 30 & & & & & & \\
\hline
\end{tabular}

- positive coefficient

- negative coefficient

"way4" - "accident location is at a 4-way intersection" indicator variable

"T" - "accident location is at a T-intersection" indicator variable

"str" _ - "road is straight" indicator variable 
Table C.6 (Continued)

\begin{tabular}{|c|c|c|c|c|c|c|}
\hline \multirow{3}{*}{ \# } & \multicolumn{6}{|c|}{ Coefficient (t-ratio) } \\
\hline & \multicolumn{2}{|c|}{ hill $\left[X_{19}\right]$} & \multicolumn{2}{|c|}{ driver $\left[X_{20}\right]$} & \multicolumn{2}{|c|}{$\operatorname{env}\left[\mathrm{X}_{20}\right]$} \\
\hline & fatality & injury & fatality & injury & fatality & injury \\
\hline \multirow{2}{*}{\multicolumn{7}{|c|}{$\frac{1}{2}$}} \\
\hline & & & & & & \\
\hline 3 & & & & & $-3.70(-7.24)$ & $-1.75(-30.4)$ \\
\hline \multicolumn{7}{|l|}{$4 a$} \\
\hline \multirow{2}{*}{\multicolumn{7}{|c|}{$\frac{4 b}{4 c}$}} \\
\hline & & & & & & \\
\hline \multicolumn{7}{|l|}{5} \\
\hline 6 & & & & & $-1.01(-4.10)$ & $-1.01(-4.10)$ \\
\hline \multirow{2}{*}{\multicolumn{7}{|c|}{7}} \\
\hline & & & & & & \\
\hline 9 & & & $3.64(3.12)$ & $1.67(14.5)$ & & \\
\hline \multicolumn{7}{|l|}{10} \\
\hline 11 & & $1.25(2.18)$ & & & & \\
\hline 12 & & & & $1.52(8.49)$ & & \\
\hline 13 & & & $1.08(2.43)$ & $1.08(2.43)$ & & \\
\hline \multicolumn{7}{|l|}{14} \\
\hline 15 & & & & & $-4.07(-7.81)$ & $-2.04(-27.3)$ \\
\hline 16 & & & $-2.50(-2.76)$ & & & \\
\hline \multicolumn{7}{|l|}{17} \\
\hline 18 & & & & & & \\
\hline \multicolumn{7}{|l|}{19} \\
\hline \multicolumn{7}{|l|}{20} \\
\hline 21 & & & & & $-1.39(-3.55)$ & $-1.39(-3.55)$ \\
\hline \multicolumn{7}{|l|}{$22 \mathrm{a}$} \\
\hline \multicolumn{7}{|l|}{$22 \mathrm{~b}$} \\
\hline \multicolumn{7}{|l|}{23} \\
\hline 24 & & & & $1.01(7.15)$ & & \\
\hline \multicolumn{7}{|l|}{25} \\
\hline \multicolumn{7}{|l|}{26} \\
\hline 27 & & & & & $-3.91(-3.79)$ & $-1.98(-17.1)$ \\
\hline \multicolumn{7}{|l|}{28} \\
\hline 29 & & & & & & \\
\hline 30 & & & $.817(2.06)$ & $.817(2.06)$ & & \\
\hline
\end{tabular}

- positive coefficient

- negative coefficient

"hill" _ _ "road is at hill" indicator variable

"driver" - "primary cause of accident is driver-related" indicator variable

"env" - "primary cause of accident is environment-related" indicator variable 
Table C.6 (Continued)

\begin{tabular}{|c|c|c|c|c|c|c|}
\hline \multirow{3}{*}{ \# } & \multicolumn{6}{|c|}{ Coefficient (t-ratio) } \\
\hline & \multicolumn{2}{|c|}{$\mathrm{hl} 5\left[\mathrm{X}_{22}\right]$} & \multicolumn{2}{|c|}{ help $\left[\mathrm{X}_{22}\right]$} & \multicolumn{2}{|c|}{$\mathrm{hl} 10\left[\mathrm{X}_{22}\right]$} \\
\hline & fatality & injury & fatality & injury & fatality & injury \\
\hline 1 & & & & & $1.00(8.01)$ & \\
\hline 2 & & & & & & \\
\hline 3 & & & & & & \\
\hline $4 a$ & & & & & & \\
\hline $4 b$ & & & & & & $.805(2.12)$ \\
\hline $4 c$ & & & & $-.0809(-3.77)$ & & \\
\hline 5 & & & & & & \\
\hline 6 & & & & & & \\
\hline 7 & & & & & & $.658(3.75)$ \\
\hline 8 & & & & & & \\
\hline 9 & & & & & & \\
\hline 10 & & & & & & \\
\hline 11 & & & & & $.905(3.76)$ & $.905(3.76)$ \\
\hline 12 & & & & & & \\
\hline 13 & & & & & & \\
\hline 14 & & & & & & \\
\hline 15 & & & & & & \\
\hline 16 & $.676(10.5)$ & $.676(10.5)$ & & & & \\
\hline 17 & & & & & & \\
\hline 18 & $.752(6.14)$ & $.752(6.14)$ & & & & \\
\hline 19 & & & & & $.567(4.93)$ & $.567(4.93)$ \\
\hline 20 & & & & & & \\
\hline 21 & & & & & & \\
\hline $22 \mathrm{a}$ & & & & & $.692(13.2)$ & $692(13.2)$ \\
\hline $22 \mathrm{~b}$ & .671(12.3) & $.671(12.3)$ & & & & \\
\hline $22 \mathrm{c}$ & & & & & . $770(.687)$ & $.770(.687)$ \\
\hline 23 & & $.184(4.69)$ & & & & \\
\hline 24 & & & & & $.867(10.5)$ & $.867(10.5)$ \\
\hline 25 & & & & & & $1.01(5.04)$ \\
\hline 26 & & $.756(2.84)$ & & & & \\
\hline 21 & & & & & & \\
\hline 28 & $.647(5.72)$ & $.647(5.72)$ & & & & \\
\hline 29 & & & $.0871(-2.93)$ & $-.0871(-2.93)$ & & \\
\hline 30 & & $.677(2.42)$ & & & & \\
\hline
\end{tabular}

- positive coefficient

- negative coefficient

"hl5" - - "help arrived in 5 minutes or less after the crash" indicator variable

"help" - "time when help arrived after the crash" indicator variable

"hl10" - "help arrived in 10 minutes or less after the crash" indicator variable 
Table C.6 (Continued)

\begin{tabular}{|c|c|c|c|c|c|c|}
\hline \multirow{3}{*}{ \# } & \multicolumn{6}{|c|}{ Coefficient (t-ratio) } \\
\hline & \multicolumn{2}{|c|}{$\mathrm{hl} 20\left[\mathrm{X}_{22}\right]$} & \multicolumn{2}{|c|}{$\operatorname{car}\left[\mathrm{X}_{25}\right]$} & \multicolumn{2}{|c|}{ moto $\left[X_{25}\right]$} \\
\hline & fatality & injury & fatality & injury & fatality & injury \\
\hline \multirow{2}{*}{\multicolumn{7}{|c|}{$\frac{1}{2}$}} \\
\hline & & & & & & \\
\hline 3 & $.910(18.7)$ & & & & $3.36(9.64)$ & $3.13(19.4)$ \\
\hline $4 a$ & $-1.19(-6.06)$ & $-1.19(-6.06)$ & & & & \\
\hline \multirow{2}{*}{\multicolumn{7}{|c|}{$\begin{array}{l}4 b \\
c\end{array}$}} \\
\hline & & & & & & \\
\hline \multicolumn{7}{|l|}{5} \\
\hline 6 & $1.06(4.31)$ & $1.06(4.31)$ & & & $2.46(5.05)$ & $2.46(5.05)$ \\
\hline \multicolumn{7}{|l|}{7} \\
\hline 8 & $1.57(4.29)$ & $1.57(4.29)$ & & & & \\
\hline 9 & $1.71(2.19)$ & $.651(5.74)$ & & & $4.58(5.63)$ & $2.89(5.44)$ \\
\hline 10 & $.578(3.80)$ & $.578(3.80)$ & & & & \\
\hline 11 & & & & $.560(2.35)$ & & \\
\hline 12 & $.929(5.83)$ & $.929(5.83)$ & & & $4.41(6.05)$ & $2.65(5.76)$ \\
\hline 13 & $1.10(4.48)$ & $1.10(4.48)$ & & & & \\
\hline \multicolumn{7}{|l|}{14} \\
\hline 15 & $.952(12.3)$ & $.952(12.3)$ & & & $3.25(16.4)$ & $3.25(16.4)$ \\
\hline \multicolumn{7}{|l|}{16} \\
\hline \multicolumn{7}{|l|}{17} \\
\hline 18 & & & & & & $2.37(6.54)$ \\
\hline \multicolumn{7}{|l|}{19} \\
\hline \multicolumn{7}{|l|}{20} \\
\hline 21 & & & & & $4.63(4.32)$ & $4.63(4.32)$ \\
\hline \multicolumn{7}{|l|}{$\frac{22 a}{22 b}$} \\
\hline \multicolumn{7}{|l|}{$22 \mathrm{c}$} \\
\hline 23 & & & & $.519(3.06)$ & & \\
\hline 24 & & & & & $2.35(9.76)$ & 2.35 (9.76) \\
\hline \multicolumn{7}{|l|}{25} \\
\hline 26 & & & & & & \\
\hline 27 & $.950(7.15)$ & .950(7.15) & & & & $3.47(8.12)$ \\
\hline \multicolumn{7}{|l|}{28} \\
\hline 29 & & & & & & \\
\hline 30 & & & & & $3.38(3.05)$ & $3.38(3.05)$ \\
\hline
\end{tabular}

- positive coefficient

- negative coefficient

"hl20" - "help arrived in 20 minutes or less after the crash" indicator variable

"car" - "the vehicle at fault is a car" indicator variable

"moto" - "the vehicle at fault is a motorcycle" indicator variable 
Table C.6 (Continued)

\begin{tabular}{|c|c|c|c|c|c|c|}
\hline \multirow{3}{*}{ \# } & \multicolumn{6}{|c|}{ Coefficient (t-ratio) } \\
\hline & \multicolumn{2}{|c|}{ pickup $\left[\mathrm{X}_{25}\right]$} & \multicolumn{2}{|c|}{ vage $\left[\mathrm{X}_{26}\right]$} & \multicolumn{2}{|c|}{ voldg $\left[X_{26}\right]$} \\
\hline & fatality & injury & fatality & injury & fatality & injury \\
\hline \multicolumn{7}{|l|}{1} \\
\hline 2 & $2.76(2.11)$ & $1.02(2.20)$ & & & & \\
\hline 3 & & & $.0376(9.76)$ & $.0376(9.76)$ & & \\
\hline \multicolumn{7}{|l|}{$4 a$} \\
\hline $4 b$ & & & & & & \\
\hline \multicolumn{7}{|l|}{$4 c$} \\
\hline \multicolumn{7}{|l|}{5} \\
\hline 6 & & & $.0543(3.24)$ & $.0543(3.24)$ & & \\
\hline \multirow{2}{*}{\multicolumn{7}{|c|}{$\frac{7}{8}$}} \\
\hline & & & & & & \\
\hline 9 & & & $.0372(3.67)$ & $.0372(3.67)$ & & \\
\hline \multicolumn{7}{|l|}{10} \\
\hline \multicolumn{7}{|l|}{11} \\
\hline 12 & & & & & $.318(2.55)$ & .318 (2.55) \\
\hline \multicolumn{7}{|l|}{13} \\
\hline \multicolumn{7}{|l|}{14} \\
\hline 15 & & & $.0373(6.55)$ & .0373 (6.55) & & \\
\hline \multicolumn{7}{|l|}{16} \\
\hline \multicolumn{7}{|l|}{17} \\
\hline \multicolumn{7}{|l|}{18} \\
\hline \multicolumn{7}{|l|}{19} \\
\hline \multirow{2}{*}{\multicolumn{7}{|c|}{$\frac{20}{21}$}} \\
\hline & & & & & & \\
\hline $22 a$ & & & & & & $.137(3.19)$ \\
\hline $\begin{array}{l}22 b \\
22 c\end{array}$ & & & & & $237(246)$ & $\begin{array}{l}.142(2.63) \\
237(2.46)\end{array}$ \\
\hline 23 & & & $.0333(2.31)$ & $.0333(2.31)$ & & \\
\hline 24 & & & $.0145(2.35)$ & $.0145(2.35)$ & & \\
\hline 25 & & & & & & \\
\hline 26 & & & & & & \\
\hline 27 & & & $.0375(4.20)$ & $.0375(4.20)$ & & \\
\hline 28 & & & & & & \\
\hline 29 & & & & & & \\
\hline 30 & & & $.0566(2.14)$ & .0566 (2.14) & & \\
\hline
\end{tabular}

- positive coefficient

- negative coefficient

"pickup" - "the vehicle at fault is a pickup" indicator variable

"vage" - "age (in years) of the vehicle at fault" quantitative variable

"voldg" - "the vehicle at fault is more than 7 years old" indicator variable 
Table C.6 (Continued)

\begin{tabular}{|c|c|c|c|c|c|c|}
\hline \multirow{3}{*}{ \# } & \multicolumn{6}{|c|}{ Coefficient (t-ratio) } \\
\hline & \multicolumn{2}{|c|}{$X_{27}$} & \multicolumn{2}{|c|}{ Ind $\left[\mathrm{X}_{28}\right]$} & \multicolumn{2}{|c|}{ othUS $\left[X_{28}\right]$} \\
\hline & fatality & injury & fatality & injury & fatality & injury \\
\hline \multirow{2}{*}{\multicolumn{7}{|c|}{$\frac{1}{2}$}} \\
\hline & & & & & & \\
\hline 3 & $.200(8.23)$ & $.200(8.23)$ & & & & \\
\hline \multicolumn{7}{|l|}{$4 a$} \\
\hline \multirow{2}{*}{\multicolumn{7}{|c|}{$4 c$}} \\
\hline & & & & & & \\
\hline 5 & $2.15(2.74)$ & $2.15(2.74)$ & & & & \\
\hline \multicolumn{7}{|c|}{$2,1,1}$, \\
\hline \multirow{2}{*}{\multicolumn{7}{|c|}{7}} \\
\hline & & & & & \multicolumn{2}{|c|}{8} \\
\hline 9 & $.385(4.20)$ & $2.89(5.44)$ & & & & \\
\hline \multicolumn{7}{|l|}{10} \\
\hline \multicolumn{7}{|l|}{$\begin{array}{l}11 \\
12\end{array}$} \\
\hline 12 & & & & & & \\
\hline \multicolumn{7}{|l|}{13} \\
\hline \multicolumn{7}{|l|}{14} \\
\hline \multicolumn{7}{|l|}{15} \\
\hline \multirow{2}{*}{\multicolumn{7}{|c|}{$\frac{16}{17}$}} \\
\hline & & & & & & \\
\hline \multicolumn{7}{|c|}{$.433(2.05)$} \\
\hline \multicolumn{7}{|l|}{19} \\
\hline \multirow{2}{*}{\multicolumn{7}{|c|}{21}} \\
\hline & & & & & & \\
\hline \multicolumn{7}{|c|}{$-.127(-3.76)$} \\
\hline \multicolumn{7}{|l|}{$22 \mathrm{~b}$} \\
\hline $22 \mathrm{c}$ & & & & & $.617(2.08)$ & $.617(2.08)$ \\
\hline \multicolumn{7}{|l|}{23} \\
\hline 24 & $105(2.66)$ & $105(2.66)$ & $.585(4.28)$ & $.585(4.28)$ & & \\
\hline \multicolumn{7}{|l|}{25} \\
\hline \multicolumn{7}{|l|}{26} \\
\hline 27 & $.415(3.83)$ & & & & $.621(2.54)$ & $.621(2.54)$ \\
\hline \multicolumn{7}{|l|}{28} \\
\hline 29 & & & & & & \\
\hline 30 & & & & & & \\
\hline
\end{tabular}

- positive coefficient

- negative coefficient

"X $\mathrm{X}_{27} \quad$ - "number of occupants in the vehicle at fault" quantitative variable

"Ind" - "license state of the vehicle at fault is Indiana" indicator variable

"othUS" - "license state of the vehicle at fault is a U.S. state except Indiana and its neighboring states (IL, $\mathrm{KY}, \mathrm{OH}, \mathrm{MI})$ " indicator variable 
Table C.6 (Continued)

\begin{tabular}{|c|c|c|c|c|c|c|}
\hline \multirow{3}{*}{ \# } & \multicolumn{6}{|c|}{ Coefficient (t-ratio) } \\
\hline & \multicolumn{2}{|c|}{ neighs $\left[\mathrm{X}_{28}\right]$} & \multicolumn{2}{|c|}{$\operatorname{lnm}\left[X_{30}\right]$} & \multicolumn{2}{|c|}{ priv $\left[\mathrm{X}_{30}\right]$} \\
\hline & fatality & injury & fatality & injury & fatality & injury \\
\hline \multicolumn{7}{|l|}{1} \\
\hline \multirow{2}{*}{\multicolumn{7}{|c|}{$\frac{2}{3}$}} \\
\hline & & & & & & \\
\hline \multicolumn{7}{|l|}{$4 a$} \\
\hline \multicolumn{7}{|l|}{$4 b$} \\
\hline \multirow{2}{*}{\multicolumn{7}{|c|}{$\frac{4 c}{5}$}} \\
\hline & & & & & & \\
\hline \multicolumn{7}{|l|}{6} \\
\hline \multirow{2}{*}{\multicolumn{7}{|c|}{$\frac{7}{8}$}} \\
\hline & & & & & & \\
\hline \multicolumn{7}{|l|}{9} \\
\hline \multicolumn{7}{|l|}{10} \\
\hline \multirow{2}{*}{\multicolumn{7}{|c|}{$\begin{array}{l}11 \\
12\end{array}$}} \\
\hline & & & & & & \\
\hline \multirow{2}{*}{\multicolumn{7}{|c|}{ 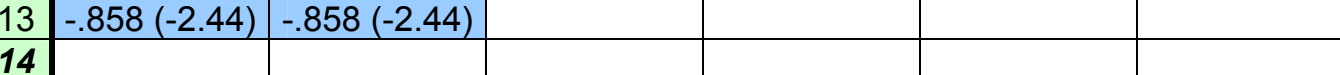 }} \\
\hline & & & & & & \\
\hline \multicolumn{7}{|l|}{15} \\
\hline \multicolumn{7}{|l|}{$\frac{16}{17}$} \\
\hline \multirow{2}{*}{\multicolumn{7}{|c|}{$\begin{array}{l}17 \\
18\end{array}$}} \\
\hline & & & & & & \\
\hline \multicolumn{7}{|l|}{19} \\
\hline \multirow{2}{*}{\multicolumn{7}{|c|}{$\begin{array}{l}20 \\
21\end{array}$}} \\
\hline & & & & & & \\
\hline $22 a$ & $-.266(-2.64)$ & $-.266(-2.64)$ & & & $-1.41(-5.03)$ & $-1.41(-5.03)$ \\
\hline $22 \mathrm{~b}$ & & & & & $-.973(-3.14)$ & $-.973(-3.14)$ \\
\hline $22 \mathrm{c}$ & & & & & $-1.49(-2.02)$ & $-1.49(-2.02)$ \\
\hline 23 & & & & & & \\
\hline 24 & & & & & & $-.616(-4.35)$ \\
\hline 25 & & & & & & \\
\hline 26 & & & & & & \\
\hline 27 & & & & & & \\
\hline 28 & & & & & & \\
\hline 29 & & & & & & \\
\hline 30 & & & $.587(2.01)$ & & & \\
\hline
\end{tabular}

- positive coefficient

- negative coefficient

"neighs" - "license state of the vehicle at fault is Indiana's neighboring states (IL, KY, OH, MI)" indicator variable

"Inm" - - "road traveled by the vehicle at fault is multi-lane" indicator variable

"priv" _ - "road traveled by the vehicle at fault is a private drive" indicator variable 
Table C.6 (Continued)

\begin{tabular}{|c|c|c|c|c|c|c|}
\hline \multirow{3}{*}{ \# } & \multicolumn{6}{|c|}{ Coefficient (t-ratio) } \\
\hline & \multicolumn{2}{|c|}{ rmu2 $\left[X_{30}\right]$} & \multicolumn{2}{|c|}{ stopsig $\left[\mathrm{X}_{31}\right]$} & \multicolumn{2}{|c|}{ nosig $\left[\mathrm{X}_{31}\right]$} \\
\hline & fatality & injury & fatality & injury & fatality & injury \\
\hline \multirow{2}{*}{\multicolumn{7}{|c|}{$\begin{array}{l}1 \\
2\end{array}$}} \\
\hline & & & & & & \\
\hline \multicolumn{7}{|l|}{3} \\
\hline \multicolumn{7}{|l|}{$4 a$} \\
\hline \multicolumn{7}{|l|}{$4 b$} \\
\hline \multicolumn{7}{|l|}{$\frac{4 c}{5}$} \\
\hline \multicolumn{7}{|l|}{5} \\
\hline 6 & $.588(1.97)$ & $.588(1.97)$ & & & & \\
\hline \multicolumn{7}{|l|}{7} \\
\hline \multirow{2}{*}{\multicolumn{7}{|c|}{8}} \\
\hline 9 & & & & & & \\
\hline \multicolumn{7}{|l|}{10} \\
\hline \multicolumn{7}{|l|}{11} \\
\hline \multicolumn{7}{|l|}{12} \\
\hline 13 & & & $.557(2.44)$ & $.557(2.44)$ & & \\
\hline \multirow{2}{*}{\multicolumn{7}{|c|}{$2.29(2.93)$}} \\
\hline & & & & & & \\
\hline \multicolumn{7}{|l|}{16} \\
\hline \multicolumn{7}{|l|}{17} \\
\hline \multicolumn{7}{|l|}{18} \\
\hline \multicolumn{7}{|c|}{19} \\
\hline \multirow{2}{*}{\multicolumn{7}{|c|}{$\frac{20}{21}$}} \\
\hline & & & & & & \\
\hline \multicolumn{7}{|c|}{$22 \mathrm{a}$} \\
\hline $22 \mathrm{~b}$ & & & & & & $-.141(-2.00)$ \\
\hline $22 \mathrm{c}$ & & & & & $-.488(-3.97)$ & $-.488(-3.97)$ \\
\hline \multicolumn{7}{|c|}{23} \\
\hline \multicolumn{7}{|c|}{24} \\
\hline \multirow{2}{*}{\multicolumn{7}{|c|}{$\frac{25}{26}$}} \\
\hline & & & & & & \\
\hline 27 & & & & & & \\
\hline 28 & & & & & & \\
\hline 29 & & & & & & \\
\hline 30 & & & & & $-.845(-2.91)$ & $-.845(-2.91)$ \\
\hline
\end{tabular}

$$
\begin{aligned}
& \ldots . \text { - positive coefficient } \\
& \ldots . \text { - negative coefficient }
\end{aligned}
$$

"rmu2" _ - "road traveled by the vehicle at fault is multi-lane AND undivided two-way" indicator variable

"stopsig" - "traffic control device for the vehicle at fault is a «stop sign»" indicator variable

"nosig" - "no any traffic control device for the vehicle at fault" indicator variable 
Table C.6 (Continued)

\begin{tabular}{|c|c|c|c|c|c|c|}
\hline \multirow{3}{*}{ \# } & \multicolumn{6}{|c|}{ Coefficient (t-ratio) } \\
\hline & \multicolumn{2}{|c|}{$\operatorname{sig}\left[X_{31}\right]$} & \multicolumn{2}{|c|}{ other $\left[X_{31}\right]$} & \multicolumn{2}{|c|}{$\operatorname{sign}\left[\mathrm{X}_{31}\right]$} \\
\hline & fatality & injury & fatality & injury & fatality & injury \\
\hline 1 & & & & & $2.14(2.04)$ & \\
\hline \multicolumn{7}{|l|}{2} \\
\hline 3 & & & & & & \\
\hline \multicolumn{7}{|l|}{$4 a$} \\
\hline \multirow{2}{*}{\multicolumn{7}{|c|}{$.848(2.28)$}} \\
\hline & & & & & & \\
\hline \multicolumn{7}{|l|}{5} \\
\hline \multicolumn{7}{|l|}{6} \\
\hline \multirow{2}{*}{\multicolumn{7}{|c|}{7}} \\
\hline \multirow{2}{*}{\multicolumn{7}{|c|}{$\frac{8}{9}$}} \\
\hline & & & & & & \\
\hline \multicolumn{7}{|l|}{10} \\
\hline \multirow{2}{*}{\multicolumn{7}{|c|}{11}} \\
\hline & & & & & & \\
\hline \multicolumn{7}{|l|}{13} \\
\hline \multicolumn{7}{|l|}{14} \\
\hline 15 & & & & & & \\
\hline \multicolumn{7}{|l|}{$\frac{15}{16}$} \\
\hline \multicolumn{7}{|l|}{17} \\
\hline \multicolumn{7}{|l|}{18} \\
\hline \multicolumn{7}{|l|}{19} \\
\hline 20 & & $1.98(2.66)$ & & & & \\
\hline 21 & $1.03(2.12)$ & $1.03(2.12)$ & & & & \\
\hline \multicolumn{7}{|l|}{$22 \mathrm{a}$} \\
\hline \multirow{2}{*}{\multicolumn{7}{|c|}{$\begin{array}{l}22 b \\
22 c\end{array}$}} \\
\hline & & & & & & \\
\hline 23 & & $487(4,25)$ & & & & \\
\hline \multicolumn{7}{|l|}{25} \\
\hline 26 & & & & & & \\
\hline \multicolumn{7}{|l|}{27} \\
\hline 28 & & & $\overline{7.18(4.14)}$ & & & \\
\hline 29 & & & & & & \\
\hline 30 & & & & & & \\
\hline
\end{tabular}

- positive coefficient

- negative coefficient

"sig" _ - "traffic control device for the vehicle at fault is a signal" indicator variable

"other" - "traffic control device for the vehicle at fault is an «other regulatory sign or marking»" indicator variable

"sign" _ _traffic control device for the vehicle at fault is an any sign" indicator variable 
Table C.6 (Continued)

\begin{tabular}{|c|c|c|c|c|c|c|}
\hline \multirow{3}{*}{ \# } & \multicolumn{6}{|c|}{ Coefficient (t-ratio) } \\
\hline & \multicolumn{2}{|c|}{ signal $\left[\mathrm{X}_{31}\right]$} & \multicolumn{2}{|c|}{$\mathrm{X}_{33}$} & \multicolumn{2}{|c|}{$X_{34}$} \\
\hline & fatality & injury & fatality & injury & fatality & injury \\
\hline \multirow{2}{*}{\multicolumn{7}{|c|}{$\frac{1}{2}$}} \\
\hline & & & & & & \\
\hline 3 & & & $2.29(6.96)$ & & $.0127(2.30)$ & \\
\hline \multicolumn{7}{|l|}{$4 a$} \\
\hline \multirow{2}{*}{\multicolumn{7}{|c|}{4}} \\
\hline & & & & & & \\
\hline \multicolumn{7}{|l|}{5} \\
\hline \multicolumn{7}{|l|}{6} \\
\hline \multirow{2}{*}{\multicolumn{7}{|c|}{$\frac{7}{8}$}} \\
\hline & & & & & & \\
\hline \multicolumn{7}{|l|}{9} \\
\hline \multicolumn{7}{|l|}{10} \\
\hline \multirow{2}{*}{\multicolumn{7}{|c|}{$\begin{array}{l}11 \\
12\end{array}$}} \\
\hline & & & & & & \\
\hline 13 & & & $2.48(2.00)$ & & $.0103(2.50)$ & $.0103(2.50)$ \\
\hline \multicolumn{7}{|r|}{. } \\
\hline 15 & & & $1.91(2.87)$ & $.793(2.11)$ & $.0153(2.24)$ & \\
\hline 16 & & & & & $.0626(3.10)$ & \\
\hline \multicolumn{7}{|l|}{17} \\
\hline \multicolumn{7}{|l|}{18} \\
\hline \multirow{2}{*}{\multicolumn{7}{|c|}{$\frac{19}{20}$}} \\
\hline & & & & & & \\
\hline 21 & & & $2.87(2.56)$ & $2.87(2.56)$ & $-.0153(-2.26)$ & $-.0153(-2.26)$ \\
\hline $22 \mathrm{a}$ & & & $1.60(4.89)$ & $1.60(4.89)$ & & \\
\hline $22 \mathrm{~b}$ & & & $2.13(5.16)$ & $2.13(5.16)$ & & \\
\hline $22 \mathrm{c}$ & & & $1.75(2.41)$ & $1.75(2.41)$ & & \\
\hline \multicolumn{7}{|l|}{23} \\
\hline 24 & & & $1.29(2.97)$ & $1.29(2.97)$ & & \\
\hline 25 & & $-.605(-2.36)$ & & & & \\
\hline \multicolumn{7}{|l|}{26} \\
\hline 27 & & & $1.76(2.24)$ & & & \\
\hline \multicolumn{7}{|l|}{28} \\
\hline 29 & & & $5.91(3.29)$ & & $.089(2.80)$ & \\
\hline 30 & & & $3.03(2.43)$ & & & \\
\hline
\end{tabular}

- positive coefficient

- negative coefficient

"signal" - "traffic control device for the vehicle at fault is an any signal" indicator variable

"X $\mathrm{X}_{33}$ " - _at least one of the vehicles involved was on fire" indicator variable

" $\mathrm{X}_{34}$ " - - "age (in years) of the driver at fault" quantitative variable 
Table C.6 (Continued)

\begin{tabular}{|c|c|c|c|c|c|c|}
\hline \multirow{3}{*}{ \# } & \multicolumn{6}{|c|}{ Coefficient (t-ratio) } \\
\hline & \multicolumn{2}{|c|}{ age $1\left[\mathrm{X}_{34}\right]$} & \multicolumn{2}{|c|}{ age2 $\left[\mathrm{X}_{34}\right]$} & \multicolumn{2}{|c|}{ age5 $\left[\mathrm{X}_{34}\right]$} \\
\hline & fatality & injury & fatality & injury & fatality & injury \\
\hline \multirow{2}{*}{\multicolumn{7}{|c|}{$\frac{1}{2}$}} \\
\hline & & & & & & \\
\hline \multicolumn{7}{|l|}{3} \\
\hline $4 a$ & & & $-1.46(-3.43)$ & $-1.46(-3.43)$ & & \\
\hline \multicolumn{7}{|c|}{$x_{1}$} \\
\hline $4 c$ & $-.789(-2.09)$ & & & & & \\
\hline \multicolumn{7}{|c|}{$\cdots 0,1-20-1$} \\
\hline \multicolumn{7}{|l|}{6} \\
\hline \multirow{2}{*}{\multicolumn{7}{|c|}{$\frac{7}{8}$}} \\
\hline & & & & & & \\
\hline \multicolumn{7}{|l|}{9} \\
\hline \multicolumn{7}{|l|}{10} \\
\hline \multirow{2}{*}{\multicolumn{7}{|c|}{$\begin{array}{l}\mathbf{1 1} \\
12\end{array}$}} \\
\hline & & & & & & \\
\hline \multicolumn{7}{|l|}{13} \\
\hline \multirow{2}{*}{\multicolumn{7}{|c|}{$\frac{\mathbf{1 4}}{15}$}} \\
\hline & & & & & & \\
\hline \multicolumn{7}{|l|}{16} \\
\hline \multirow{2}{*}{\multicolumn{7}{|c|}{17}} \\
\hline 18 & & & & & & \\
\hline \multicolumn{7}{|l|}{19} \\
\hline \multicolumn{7}{|l|}{20} \\
\hline \multicolumn{7}{|l|}{$\frac{21}{22 a}$} \\
\hline \multirow{2}{*}{\multicolumn{7}{|c|}{$22 \mathrm{~b}$}} \\
\hline \multirow{2}{*}{\multicolumn{7}{|c|}{$\frac{22 c}{23}$}} \\
\hline & & & & & & \\
\hline \multicolumn{7}{|c|}{$.291(3.46)$} \\
\hline \multirow{2}{*}{\multicolumn{7}{|c|}{$\frac{25}{26}$}} \\
\hline & & & & & & \\
\hline \multicolumn{7}{|l|}{27} \\
\hline \multicolumn{7}{|l|}{28} \\
\hline 29 & & & & & & \\
\hline 30 & & & & & & \\
\hline
\end{tabular}

- positive coefficient

- negative coefficient

"age1" _ - "age of the driver at fault is $\geq 18$ and $<24$ " indicator variable

"age2" - "age of the driver at fault is $\geq 24$ and $<30$ " indicator variable

"age5" - "age of the driver at fault is $\geq 50$ years" indicator variable 
Table C.6 (Continued)

\begin{tabular}{|c|c|c|c|c|c|c|}
\hline \multirow{3}{*}{ \# } & \multicolumn{6}{|c|}{ Coefficient (t-ratio) } \\
\hline & \multicolumn{2}{|c|}{$X_{35}$} & \multicolumn{2}{|c|}{$\mathrm{v} 3 \mathrm{o}\left[\mathrm{X}_{26}\right]$} & \multicolumn{2}{|c|}{ voldo $\left[X_{26}\right]$} \\
\hline & fatality & injury & fatality & injury & fatality & injury \\
\hline \multirow{2}{*}{\multicolumn{7}{|c|}{1}} \\
\hline & \\
\hline 3 & & $.226(4.92)$ & & & & \\
\hline \multicolumn{7}{|l|}{$4 a$} \\
\hline \multirow{2}{*}{\multicolumn{7}{|c|}{$\frac{4 b}{4 c}$}} \\
\hline & & & & & & \\
\hline \multicolumn{7}{|l|}{5} \\
\hline 6 & $.439(2.22)$ & $.439(2.22)$ & & & & \\
\hline 7 & $.356(2.02)$ & $.356(2.02)$ & & & & \\
\hline 8 & & & & & & $.735(2.96)$ \\
\hline \multicolumn{7}{|l|}{9} \\
\hline 10 & & $.450(3.55)$ & & & & \\
\hline \multicolumn{7}{|l|}{11} \\
\hline 12 & & $.444(3.59)$ & & & & \\
\hline \multicolumn{7}{|l|}{13} \\
\hline \multicolumn{7}{|l|}{14} \\
\hline 15 & & $.330(5.16)$ & & & & \\
\hline 16 & & & & $-.332(-3.14)$ & & \\
\hline 17 & $.892(3.16)$ & $.892(3.16)$ & & & & \\
\hline 18 & & $.483(3.86)$ & & & & \\
\hline \multicolumn{7}{|l|}{19} \\
\hline \multicolumn{7}{|l|}{20} \\
\hline 21 & & & & & & \\
\hline \multicolumn{7}{|l|}{$22 \mathrm{a}$} \\
\hline \multicolumn{7}{|l|}{$22 \mathrm{~b}$} \\
\hline \multicolumn{7}{|l|}{$22 c$} \\
\hline \multicolumn{7}{|c|}{$278(393)$} \\
\hline$\frac{24}{25}$ & $139(232)$ & $\frac{.278(3.93)}{120(2.20)}$ & & & & \\
\hline 26 & & & & & $.625(2.65)$ & $.625(2.65)$ \\
\hline 27 & $.293(2.91)$ & $.293(2.91)$ & & & & \\
\hline 28 & $-.537(-3.57)$ & $-.537(-3.57)$ & & & & \\
\hline 29 & & & & & & \\
\hline 30 & & & & & & \\
\hline
\end{tabular}

- positive coefficient

- negative coefficient

"X $\mathrm{X}_{35}$ " - "gender of the driver at fault: 1 - female, 0 - male" indicator variable

"v3o" - " "age of the oldest vehicle involved is $>1$ and $\leq 3$ years" indicator variable

"voldo" - - "age of the oldest vehicle involved is $>7$ years" indicator variable 
Table C.6 (Continued)

\begin{tabular}{|c|c|c|c|c|c|c|}
\hline \multirow{3}{*}{ \# } & \multicolumn{6}{|c|}{ Coefficient (t-ratio) } \\
\hline & \multicolumn{2}{|c|}{$\operatorname{maxpass}\left[\mathrm{X}_{27}\right]$} & \multicolumn{2}{|c|}{ age2y $\left[\mathrm{X}_{34}\right]$} & \multicolumn{2}{|c|}{ olddrv $\left[\mathrm{X}_{34}\right]$} \\
\hline & fatality & injury & fatality & injury & fatality & injury \\
\hline 1 & $.457(2.58)$ & $182(3.14)$ & & & & \\
\hline \multirow{2}{*}{\multicolumn{7}{|c|}{$\frac{2}{3}$}} \\
\hline & & & & & & \\
\hline \multirow{2}{*}{\multicolumn{7}{|c|}{$4 b$}} \\
\hline & & & & & & \\
\hline \multicolumn{7}{|l|}{$4 c$} \\
\hline \multirow{2}{*}{\multicolumn{7}{|c|}{$\begin{array}{c}5 \\
6\end{array}$}} \\
\hline & & & & & & \\
\hline \multicolumn{7}{|l|}{7} \\
\hline \multirow{2}{*}{\multicolumn{7}{|c|}{$\frac{8}{9}$}} \\
\hline & & & & & & \\
\hline 10 & $.140(2.68)$ & $.140(2.68)$ & & & & \\
\hline \multicolumn{7}{|l|}{11} \\
\hline \multicolumn{7}{|l|}{13} \\
\hline \multicolumn{7}{|l|}{14} \\
\hline \multicolumn{7}{|l|}{15} \\
\hline 16 & & $.138(4.77)$ & & & & \\
\hline \multicolumn{7}{|l|}{17} \\
\hline \multicolumn{7}{|l|}{18} \\
\hline 19 & & $.290(5.67)$ & & & & \\
\hline \multicolumn{7}{|l|}{20} \\
\hline \multicolumn{7}{|l|}{21} \\
\hline $22 a$ & & $.275(9.91)$ & & & & \\
\hline $22 \mathrm{~b}$ & $.143(5.93)$ & $.143(5.93)$ & & & & \\
\hline $22 \mathrm{c}$ & $.128(3.22)$ & $.128(3.22)$ & & & & \\
\hline 23 & & & $.512(2.76)$ & $.512(2.76)$ & & \\
\hline 24 & & & & & & \\
\hline 25 & & & & & $.133(2.40)$ & $.133(2.40)$ \\
\hline 26 & & & & & & \\
\hline 27 & & & & & & \\
\hline 28 & $.133(2.61)$ & & & & & \\
\hline 29 & & & & & & \\
\hline 30 & & & & & & \\
\hline
\end{tabular}

... - positive coefficient

- negative coefficient

\footnotetext{
"maxpass" - "the largest number of occupants in all vehicles involved" quantitative variable

"age2y" - "age of the youngest driver is $\geq 24$ and $<30$ years" indicator variable

"olddrv" _ - "the driver at fault is older than the other driver involved" indicator variable
} 
Table C.6 (Continued)

\begin{tabular}{|c|c|c|c|c|c|c|}
\hline \multirow{3}{*}{ \# } & \multicolumn{6}{|c|}{ Coefficient (t-ratio) } \\
\hline & \multicolumn{2}{|c|}{ age0o $\left[\mathrm{X}_{34}\right]$} & \multicolumn{2}{|c|}{ age2o $\left[\mathrm{X}_{34}\right]$} & \multicolumn{2}{|c|}{ age4o $\left[\mathrm{X}_{34}\right]$} \\
\hline & fatality & injury & fatality & injury & fatality & injury \\
\hline \multirow{2}{*}{\multicolumn{7}{|c|}{1}} \\
\hline & & & & & & \\
\hline \multicolumn{7}{|l|}{3} \\
\hline \multicolumn{7}{|l|}{$4 a$} \\
\hline \multirow{2}{*}{\multicolumn{7}{|c|}{$\begin{array}{l}4 b \\
4 c\end{array}$}} \\
\hline & & & & & & \\
\hline \multicolumn{7}{|l|}{5} \\
\hline \multicolumn{7}{|l|}{6} \\
\hline 7 & & & $1.75(1.99)$ & & & \\
\hline \multicolumn{7}{|c|}{ ……․ } \\
\hline \multicolumn{7}{|l|}{9} \\
\hline \multirow{2}{*}{\multicolumn{7}{|c|}{$\frac{10}{11}$}} \\
\hline & & & & & & \\
\hline \multicolumn{7}{|l|}{12} \\
\hline \multicolumn{7}{|l|}{13} \\
\hline \multirow{2}{*}{\multicolumn{7}{|c|}{$\frac{14}{15}$}} \\
\hline & & & & & & \\
\hline 16 & & & & & & $214(2.93)$ \\
\hline \multicolumn{7}{|l|}{17} \\
\hline \multicolumn{7}{|l|}{18} \\
\hline \multirow{2}{*}{\multicolumn{7}{|c|}{\begin{tabular}{l|}
19 \\
20
\end{tabular}}} \\
\hline & & & & & & \\
\hline \multicolumn{7}{|l|}{21} \\
\hline \multicolumn{7}{|c|}{$-1.25(-4.40)$} \\
\hline $22 \mathrm{~b}$ & $-2.20(-3.03)$ & $-2.20(-3.03)$ & & & & \\
\hline \multirow{2}{*}{\multicolumn{5}{|c|}{$\frac{2<c}{23}$}} & & \\
\hline \multirow{2}{*}{\multicolumn{7}{|c|}{24}} \\
\hline & & & & & & \\
\hline \multicolumn{7}{|l|}{25} \\
\hline \multirow{2}{*}{\multicolumn{7}{|c|}{$\begin{array}{l}26 \\
27\end{array}$}} \\
\hline & & & & & & \\
\hline 28 & & & & & & \\
\hline 29 & & & & & & \\
\hline 30 & & & & & & \\
\hline
\end{tabular}

- positive coefficient

- negative coefficient

"age0o" - "age of the oldest driver is $<18$ years" indicator variable

"age2o" - "age of the oldest driver is $\geq 24$ and $<30$ years" indicator variable

"age4o" - "age of the oldest driver is $\geq 40$ and $<50$ years " indicator variable 
Table C.6 (Continued)

\begin{tabular}{|c|c|c|c|c|c|c|}
\hline \multirow{3}{*}{ \# } & \multicolumn{6}{|c|}{ Coefficient (t-ratio) } \\
\hline & \multicolumn{2}{|c|}{$\mathrm{ff}\left[\mathrm{X}_{35}\right]$} & \multicolumn{2}{|c|}{$\mathrm{mm}\left[\mathrm{X}_{35}\right]$} & \multicolumn{2}{|c|}{$\mathrm{mf}\left[\mathrm{X}_{35}\right]$} \\
\hline & fatality & injury & fatality & injury & fatality & injury \\
\hline \multicolumn{7}{|l|}{1} \\
\hline \multicolumn{7}{|l|}{2} \\
\hline \multirow{2}{*}{\multicolumn{7}{|c|}{$\frac{3}{4 a}$}} \\
\hline & & & & & & \\
\hline \multicolumn{7}{|l|}{$4 b$} \\
\hline \multirow{2}{*}{\multicolumn{7}{|c|}{$.0<(2.04)$}} \\
\hline & & & & & & \\
\hline \multicolumn{7}{|l|}{6} \\
\hline \multirow{2}{*}{\multicolumn{7}{|c|}{7}} \\
\hline \multirow{2}{*}{\multicolumn{7}{|c|}{$\frac{8}{9}$}} \\
\hline & & & & & & \\
\hline \multicolumn{7}{|l|}{10} \\
\hline \multirow{2}{*}{\multicolumn{7}{|c|}{$.534(2.25)$}} \\
\hline & & & & & & \\
\hline \multicolumn{7}{|l|}{13} \\
\hline \multicolumn{7}{|l|}{$\frac{14}{15}$} \\
\hline \multicolumn{7}{|l|}{15} \\
\hline 16 & & & & $-.240(-3.39)$ & & \\
\hline \multicolumn{7}{|c|}{$.40(0.00)$} \\
\hline \multicolumn{7}{|l|}{18} \\
\hline \multirow{2}{*}{\multicolumn{7}{|c|}{\begin{tabular}{l|}
19 \\
20
\end{tabular}}} \\
\hline & & & & & & \\
\hline \multicolumn{7}{|l|}{21} \\
\hline \multicolumn{7}{|c|}{$-.217(-4.11)$} \\
\hline \multicolumn{7}{|c|}{$-.193(-3.13)$} \\
\hline \multirow{2}{*}{\multicolumn{7}{|c|}{ 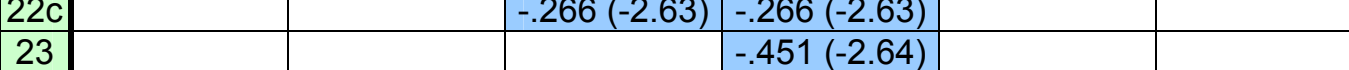 }} \\
\hline & & & & $-.451(-2.64)$ & & \\
\hline \multicolumn{7}{|l|}{24} \\
\hline \multicolumn{7}{|l|}{25} \\
\hline 26 & & & & & & \\
\hline$\frac{27}{20}$ & & & & & & \\
\hline 28 & $.725(4.24)$ & $.725(4.24)$ & & & & \\
\hline $\begin{array}{l}29 \\
30\end{array}$ & & & & & & \\
\hline & & & & & & \\
\hline
\end{tabular}

- positive coefficient

- negative coefficient

"ff" - "two female drivers involved into a two-vehicle accident" indicator variable

"mm" - "two male drivers involved into a two-vehicle accident" indicator variable

"mf" - "male and female drivers involved into a two-vehicle accident" indicator variable 
Table C.7 Tests of car-SUV separation in 2004 accident severity study ${ }^{24}$

\begin{tabular}{|c|c|c|c|c|c|c|c|c|c|c|}
\hline \# & & & Model name & $M$ & K & $L L\left(\boldsymbol{\beta}_{\mathbf{m}}\right)$ & $\sum L L\left(\boldsymbol{\beta}_{\mathbf{m}}\right)$ & $d f$ & $p$-value & conclusion* \\
\hline 1 & \multirow{2}{*}{$\begin{array}{l}0 \\
\mathbb{\pi} \\
\stackrel{0}{0}\end{array}$} & \multirow{2}{*}{ 풀 } & (car/SUV)+(car/SUV) & 3 & 17 & -1636.3 & -1616.3 & 34 & 0.22 & Car $=$ SUV \\
\hline 2 & & & (car/SUV)+(truck) & 2 & 9 & -250.47 & -248.11 & 9 & 0.86 & Car $=$ SUV \\
\hline 4 & \multirow[b]{2}{*}{ ن } & \multirow{2}{*}{$\begin{array}{l}\frac{c}{\sqrt{0}} \\
\frac{0}{\grave{J}}\end{array}$} & (car/SUV)+(car/SUV) & 3 & 10 & -683.95 & -678.39 & 20 & 0.94 & Car $=$ SUV \\
\hline 5 & & & (car/SUV)+(truck) & 2 & 5 & -104.80 & -100.71 & 5 & 0.15 & Car $=$ SUV \\
\hline 7 & \multirow{4}{*}{ 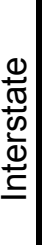 } & \multirow{2}{*}{$\overline{\frac{\pi}{2}}$} & (car/SUV)+(car/SUV) & 3 & 4 & -456.77 & -451.09 & 8 & 0.18 & Car $=$ SUV \\
\hline 8 & & & (car/SUV)+(truck) & 2 & 9 & -318.00 & -308.75 & 9 & 0.03 & Car $\neq$ SUV \\
\hline 10 & & \multirow{2}{*}{$\begin{array}{l}\frac{c}{\widetilde{D}} \\
\frac{0}{\zeta} \\
\frac{1}{J}\end{array}$} & $(\mathrm{car} / \mathrm{SUV})+(\mathrm{car} / \mathrm{SUV})$ & 3 & 13 & -710.09 & -699.64 & 26 & 0.75 & Car $=$ SUV \\
\hline 11 & & & (car/SUV)+(truck) & 2 & 6 & -336.87 & -334.52 & 6 & 0.58 & Car $=$ SUV \\
\hline 13 & \multirow{2}{*}{\multicolumn{2}{|c|}{ 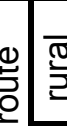 }} & $(\mathrm{car} / \mathrm{SUV})+(\mathrm{car} / \mathrm{SUV})$ & 3 & 12 & -1302.1 & -1292.1 & 24 & 0.69 & Car $=$ SUV \\
\hline 14 & & & (car/SUV)+(truck) & 2 & 11 & -362.86 & -359.27 & 11 & 0.78 & Car $=$ SUV \\
\hline 16 & \multirow{2}{*}{$\frac{\Phi}{\stackrel{0}{\pi}}$} & \multirow{2}{*}{$\begin{array}{l}\frac{c}{\widetilde{D}} \\
\frac{0}{3}\end{array}$} & (car/SUV)+(car/SUV) & 3 & 12 & -1649.1 & -1630.5 & 24 & 0.04 & Car $\neq$ SUV \\
\hline 17 & & & (car/SUV)+(truck) & 2 & 5 & -173.40 & -172.27 & 5 & 0.81 & Car $=$ SUV \\
\hline 19 & \multirow{4}{*}{ 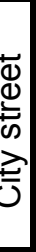 } & \multirow{2}{*}{$\begin{array}{l}\overline{\sqrt{0}} \\
\frac{2}{2}\end{array}$} & $(\mathrm{car} / \mathrm{SUV})+(\mathrm{car} / \mathrm{SUV})$ & 3 & 12 & -1358.7 & -1353.1 & 24 & 0.99 & Car $=$ SUV \\
\hline 20 & & & (car/SUV)+(truck) & 2 & 9 & -68.07 & -64.80 & 9 & 0.69 & Car $=$ SUV \\
\hline 22 & & \multirow{2}{*}{ 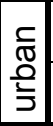 } & $(\mathrm{car} / \mathrm{SUV})+(\mathrm{car} / \mathrm{SUV})$ & 3 & 23 & -14547 & -14524 & 46 & 0.53 & Car $=$ SUV \\
\hline 23 & & & (car/SUV)+(truck) & 2 & 10 & -885.98 & -877.16 & 10 & 0.06 & Car $\neq$ SUV \\
\hline 25 & \multirow{4}{*}{ 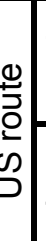 } & \multirow{2}{*}{$\overline{\frac{\pi}{2}}$} & $(\mathrm{car} / \mathrm{SUV})+(\mathrm{car} / \mathrm{SUV})$ & 3 & 11 & -978.44 & -969.45 & 22 & 0.71 & Car $=$ SUV \\
\hline 26 & & & (car/SUV)+(truck) & 2 & 12 & -270.09 & -262.03 & 12 & 0.19 & Car $=$ SUV \\
\hline 28 & & \multirow{2}{*}{$\begin{array}{l}\frac{\complement}{\sqrt{0}} \\
\frac{0}{\partial}\end{array}$} & $(\mathrm{car} / \mathrm{SUV})+(\mathrm{car} / \mathrm{SUV})$ & 3 & 12 & -2345.1 & -2334.3 & 24 & 0.60 & Car $=$ SUV \\
\hline 29 & & & (car/SUV)+(truck) & 2 & 9 & -311.62 & -305.06 & 9 & 0.16 & Car $=$ SUV \\
\hline
\end{tabular}

* For models 8, 16 and 23 we find that "Car $\neq$ SUV", which means that for these models cars and SUVs must be considered separately in our accident severity study. For all other models we find that "Car = SUV", which means that cars and SUVs can be considered together.

${ }^{24}$ For explanation of these tests refer to footnote 21 on page 106. 
Table C.8 Tests of car-SUV separation in 2006 accident severity study

\begin{tabular}{|c|c|c|c|c|c|c|c|c|c|c|}
\hline \# & & & Model name & $M$ & K & $L L\left(\boldsymbol{\beta}_{\mathbf{m}}\right)$ & $\sum L L\left(\boldsymbol{\beta}_{\mathbf{m}}\right)$ & $d f$ & $p$-value & conclusion* $^{*}$ \\
\hline \multirow{2}{*}{\multicolumn{2}{|c|}{ 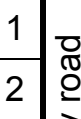 }} & \multirow{2}{*}{ 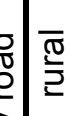 } & (car/SUV)+(car/SUV) & 3 & 10 & -832.13 & -826.10 & 20 & 0.91 & Car $=$ SUV \\
\hline & & & (car/SUV)+(truck) & 2 & 8 & -113.99 & -109.77 & 8 & 0.39 & Car $=$ SUV \\
\hline \multirow{2}{*}{$\begin{array}{l}4 \\
5\end{array}$} & \multirow{2}{*}{ 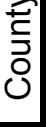 } & \multirow{2}{*}{$\begin{array}{l}\frac{}{\widetilde{\pi}} \\
\frac{0}{\grave{J}}\end{array}$} & $(\mathrm{car} / \mathrm{SUV})+(\mathrm{car} / \mathrm{SUV})$ & 3 & 2 & -782.85 & -777.77 & 4 & 0.04 & Car $\neq$ SUV \\
\hline & & & (car/SUV)+(truck) & 2 & 4 & -16.716 & -13.652 & 4 & 0.19 & Car = SUV \\
\hline \multirow[b]{2}{*}{8} & \multirow{4}{*}{ 竧 } & \multirow{2}{*}{ 준 } & $(\mathrm{car} / \mathrm{SUV})+(\mathrm{car} / \mathrm{SUV})$ & 3 & 9 & -459.31 & -447.45 & 18 & 0.16 & Car $=$ SUV \\
\hline & & & (car/SUV)+(truck) & 2 & 6 & -271.78 & -269.97 & 6 & 0.73 & Car $=$ SUV \\
\hline 10 & & $\frac{c}{\sqrt{d}}$ & $(\mathrm{car} / \mathrm{SUV})+(\mathrm{car} / \mathrm{SUV})$ & 3 & 9 & -853.29 & -846.69 & 18 & 0.78 & Car $=$ SUV \\
\hline 11 & & 은 & (car/SUV)+(truck) & 2 & 9 & -252.55 & -246.81 & 9 & 0.24 & Car $=$ SUV \\
\hline 13 & & \multirow{2}{*}{ 㞼 } & $(\mathrm{car} / \mathrm{SUV})+(\mathrm{car} / \mathrm{SUV})$ & 3 & 11 & -560.40 & -550.05 & 22 & 0.54 & Car $=$ SUV \\
\hline 14 & & & (car/SUV)+(truck) & 2 & 5 & -72.403 & -71.805 & 5 & 0.95 & Car $=$ SUV \\
\hline 16 & \multirow{2}{*}{$\frac{0}{\stackrel{0}{\pi}}$} & \multirow{2}{*}{$\begin{array}{l}\frac{c}{\widetilde{0}} \\
\frac{0}{5}\end{array}$} & (car/SUV)+(car/SUV) & 3 & 12 & -3225.9 & -3212.7 & 24 & 0.34 & Car $=$ SUV \\
\hline 17 & & & (car/SUV)+(truck) & 2 & 5 & -192.97 & -192.09 & 5 & 0.88 & Car $=$ SUV \\
\hline 19 & \multirow{4}{*}{ 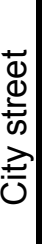 } & \multirow{2}{*}{$\begin{array}{l}\overline{\frac{\pi}{0}} \\
\frac{\stackrel{2}{2}}{2} \\
\end{array}$} & $(\mathrm{car} / \mathrm{SUV})+(\mathrm{car} / \mathrm{SUV})$ & 3 & 8 & -1068.2 & -1061.9 & 16 & 0.67 & Car $=$ SUV \\
\hline 20 & & & (car/SUV)+(truck) & 2 & 2 & -146.45 & -145.98 & 2 & 0.63 & Car $=$ SUV \\
\hline 22 & & \multirow{2}{*}{$\begin{array}{l}\frac{c}{\sqrt{0}} \\
\frac{0}{J} \\
\frac{0}{J}\end{array}$} & $(\mathrm{car} / \mathrm{SUV})+(\mathrm{car} / \mathrm{SUV})$ & 3 & 17 & -9505.6 & -9481.4 & 34 & 0.05 & Car $\neq$ SUV \\
\hline 23 & & & (car/SUV)+(truck) & 2 & 12 & -520.63 & -517.29 & 12 & 0.88 & Car $=$ SUV \\
\hline 25 & \multirow{4}{*}{ 兽 } & \multirow{2}{*}{$\frac{\overline{0}}{\stackrel{0}{2}}$} & (car/SUV)+(car/SUV) & 3 & 9 & -380.51 & -367.95 & 18 & 0.12 & Car $=$ SUV \\
\hline 26 & & & (car/SUV)+(truck) & 2 & 8 & -237.97 & -231.83 & 8 & 0.14 & Car $=$ SUV \\
\hline 28 & & \multirow{2}{*}{ 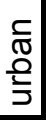 } & (car/SUV)+(car/SUV) & 3 & 7 & -967.22 & -961.02 & 14 & 0.57 & Car $=$ SUV \\
\hline 29 & & & (car/SUV)+(truck) & 2 & 7 & -185.87 & -182.84 & 7 & 0.53 & Car $=$ SUV \\
\hline
\end{tabular}

* For models 4 and 22 we find that "Car $\neq$ SUV", which means that for these models cars and SUVs must be considered separately in our accident severity study. For all other models we find that "Car = SUV", which means that cars and SUVs can be considered together. 